\title{
SEMIBOUNDED REPRESENTATIONS AND INVARIANT CONES IN INFINITE DIMENSIONAL LIE ALGEBRAS
}

\author{
KARL-HERMANN NEEB \\ Fachbereich Mathematik, TU Darmstadt \\ Schlossgartenstrasse 7, 64289-Darmstadt, Germany \\ neeb@mathematik.tu-darmstadt.de \\ Received 23 November 2009 \\ Revised 16 December 2009
}

\begin{abstract}
A unitary representation of a, possibly infinite dimensional, Lie group $G$ is called semibounded if the corresponding operators $i \mathrm{~d} \pi(x)$ from the derived representations are uniformly bounded from above on some non-empty open subset of the Lie algebra $\mathfrak{g}$. In the first part of the present paper we explain how this concept leads to a fruitful interaction between the areas of infinite dimensional convexity, Lie theory, symplectic geometry (momentum maps) and complex analysis. Here open invariant cones in Lie algebras play a central role and semibounded representations have interesting connections to $C^{*}$-algebras which are quite different from the classical use of the group $C^{*}$-algebra of a finite dimensional Lie group. The second half is devoted to a detailed discussion of semibounded representations of the diffeomorphism group of the circle, the Virasoro group, the metaplectic representation on the bosonic Fock space and the spin representation on fermionic Fock space.
\end{abstract}

Keywords: Infinite dimensional Lie group; unitary representation; momentum map; momentum set; semibounded representation; metaplectic representation; spin representation; Virasoro algebra.

AMS Subject Classification: 22E65, 22E45

\section{Contents}

1. Introduction

2. Semi-Equicontinuous Convex Sets 45

3. Infinite Dimensional Lie Groups 49

4. Momentum Sets of Smooth Unitary Representations 55

5. Aspects of Complex Analysis 61

5.1. The Abelian case . . . . . . . . . . . . . . . . . . . . . . . 62

5.2. Hilbert spaces of holomorphic functions . . . . . . . . . 63

6. Invariant Cones in Lie Algebras 69

6.1. Invariant cones in unitary Lie algebras . . . . . . . . . . . . . . 69

6.2. Invariant cones in symplectic Lie algebras . . . . . . . . . . . . 69 
6.3. Invariant Lorentzian cones . . . . . . . . . . . . . . . . . . . 73

6.4. Invariant cones of vector fields . . . . . . . . . . . . . . . . 74

6.5. Invariant cones and symmetric Hilbert domains . . . . . . . . . . 75

6.6. A general lemma . . . . . . . . . . . . . . . . 76

$\begin{array}{ll}\text { 7. Connections to } C^{*} \text {-Algebras } & 77\end{array}$

7.1. Representations of the unitary group . . . . . . . . . . . . . . 77

7.2. $C^{*}$-dynamical systems . . . . . . . . . . . . . . . . . 79

8. The Virasoro Algebra and Vector Fields on $\mathbb{S}^{1} \quad 81$

8.1. The invariant cones in $\mathcal{V}\left(\mathbb{S}^{1}\right) \ldots \ldots$. . . . . . . . . . . . . . . 82

8.2. Invariant cones in the Virasoro algebra . . . . . . . . . . . . . 88

8.3. Semi-equicontinuity of coadjoint orbits of $\mathfrak{v i r}^{\prime} \ldots$. . . . . . . . . 94

9. Symplectic Group and Metaplectic Representation $\quad 97$

9.1. The metaplectic representation . . . . . . . . . . . . . . . . 98

9.2. The metaplectic group . . . . . . . . . . . . . . . . . 101

9.3. Semiboundedness of the metaplectic representation . . . . . . . . . 103

9.4. The momentum set of the metaplectic representation . . . . . . . . 107

10. The Spin Representation 110

10.1. Semiboundedness of the spin representation . . . . . . . . . . 110

10.2. Quasi-free representations . . . . . . . . . . . 117

11. Perspectives 118

A. Smooth Vectors for Representations 121

B. The Cone of Positive Definite Forms on a Banach Space 123

C. Involutive Lie Algebras with Root Decomposition 125

D. Some Facts on Fock Spaces $\quad 126$

\section{Glossary}

$\left(\pi_{a}, \Lambda(\mathcal{H})\right)$ spin representation of metagonal group, 111

$\left(\pi_{s}, S(\mathcal{H})\right)$ metaplectic representation of metapl. group, 99

$A \ll B \quad$ inf $\operatorname{Spec}(B-A)>0$, for $A, B \in \operatorname{Herm}(\mathcal{H}), 60$

$B(X) \quad=\{v \in E: \inf \langle X, v\rangle>-\infty\}$ for $X \subseteq E^{\prime}, 45$

$B_{p}(\mathcal{H}) \quad$ Schatten ideal of order $p, 51$

$C^{\star} \quad$ dual cone of $C, 46$

$C_{\pi} \quad$ interior of $\left(I_{\pi}\right)^{\star}$, for unit. rep. $\pi, 58$

$C_{\mathfrak{u}(\mathcal{H})}=\{x \in \mathfrak{u}(\mathcal{H}): i x \ll 0\}, 69$

$H(C) \quad$ translations preserving convex set $C, 46$

$H_{X}(v) \quad=\frac{1}{2} \omega(X v, v)$, hamilt. funct., $X \in \mathfrak{s p}(V, \omega), 69$

$I_{\pi} \quad \overline{\operatorname{conv}}\left(\operatorname{im}\left(\Phi_{\pi}\right)\right)$, momentum set of $\pi, 56$

$S(\mathcal{A}) \quad$ states of the $C^{*}$-algebra $\mathcal{A}, 77$

$S(\mathcal{H}) \quad$ bosonic/symmetric Fock space, 60

$W_{\pi} \quad=B\left(I_{\pi}\right)^{0}$, for unit. rep. $\pi, 58$ 


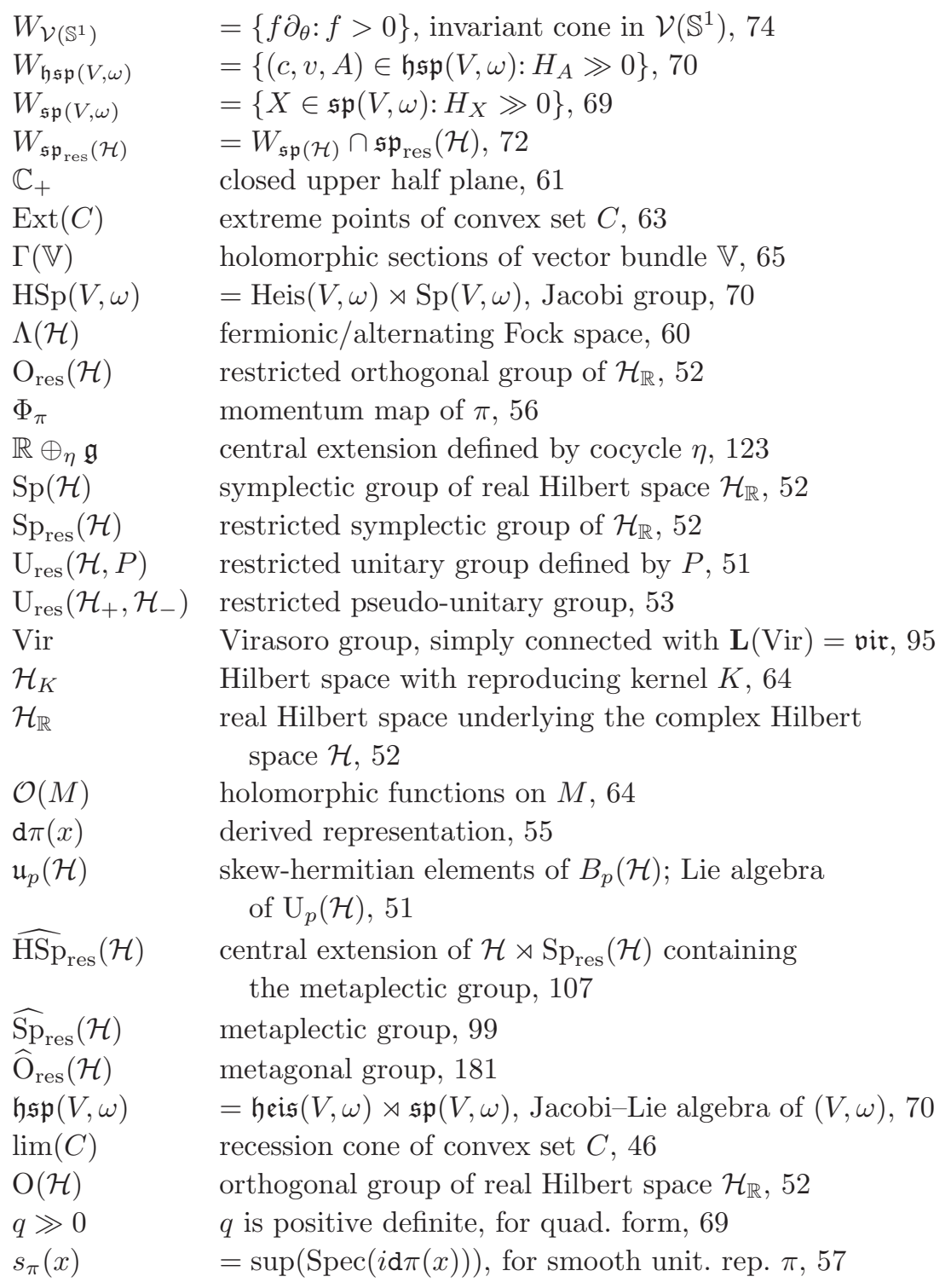

\section{Introduction}

In the unitary representation theory of a finite dimensional Lie group $G$ a central tool is the convolution algebra $L^{1}(G)$, resp., its enveloping $C^{*}$-algebra $C^{*}(G)$, whose construction is based on the Haar measure, whose existence follows from the local compactness of $G$. Since the non-degenerate representations of $C^{*}(G)$ are in oneto-one correspondence to continuous unitary representations of $G$, the full power of the rich theory of $C^{*}$-algebras can be used to study unitary representations of $G$. 
To understand and classify irreducible unitary representations, the crucial methods are usually based on the fine structure theory of finite dimensional Lie groups, such as Levi and Iwasawa decompositions. Both methods are no longer available for infinite dimensional Lie groups because they are not locally compact and there is no general structure theory available.

However, there are many interesting classes of infinite dimensional Lie groups which possess a rich unitary representation theory. Many of these representations show up naturally in various contexts of mathematical physics ([15, 55, 56, 81, 88, $18,90,94,4])$. The representations arising in mathematical physics, resp., Quantum Field Theory are often characterized by the requirement that the Lie algebra $\mathfrak{g}=\mathbf{L}(G)$ of $G$ contains an element $h$, corresponding to the Hamiltonian of the underlying physical system, for which the spectrum of the operator $i \cdot \mathrm{d} \pi(h)$ in the "physically relevant" representations $(\pi, \mathcal{H})$ is non-negative. These representations are called positive energy representations (cf. [92,10,88,21]).

To develop a reasonably general powerful theory of unitary representations of infinite dimensional Lie groups, new approaches have to be developed which do neither rest on a fine structure theory nor on the existence of invariant measures. In this note we describe a systematic approach which is very much inspired by the concepts and requirements of mathematical physics and which provides a unifying framework for a substantial class of representations and several interesting phenomena. Due to the lack of a general structure theory, one has to study specific classes of representations. Here we focus on semibounded representations. Semiboundedness is a stable version of the positive energy condition. It means that the self-adjoint operators $i \mathrm{~d} \pi(x)$ from the derived representation are uniformly bounded below for all $x$ in some non-empty open subset of $\mathfrak{g}$. Our long term goal is to understand the decomposition theory and the irreducible semibounded representations by their geometric realizations.

The theory of semibounded unitary representations combines results, concepts and methods from several branches of mathematics: the theory of convex sets and functions in locally convex spaces, infinite dimensional Lie theory, symplectic geometry (momentum maps, coadjoint orbits) and complex geometry (infinite dimensional Kähler manifolds and complex semigroup actions). In Secs. 2-5 below, we describe the relevant aspects of these four areas and recall some basic results from $[67,68]$. A crucial new point is that our approach provides a common functional analytic environment for various important classes of unitary representations of infinite dimensional Lie groups. That it is now possible to study semibounded representations in this generality is due to the recent progress in infinite dimensional Lie theory with fundamental achievements in the past decade. For a detailed survey we refer to [66]. A comprehensive exposition of the theory will soon be available in our monograph with Glöckner [25].

For finite dimensional Lie groups semibounded unitary representations are well understood. In [60] they are called "generalized highest weight representations" because the irreducible ones permit a classification in terms of their highest weight 
with respect to a root decomposition of a suitable quotient algebra (see Remark 5.5 for more details on this case). This simple picture does not carry over to infinite dimensional groups.

We now describe our setting in some more detail. Based on the notion of a smooth map between open subsets of a locally convex space one obtains the concept of a locally convex manifold and hence of a locally convex Lie group (cf. $[66,57,25]$ ). In Sec. 3, we discuss some key examples. Let $G$ be a (locally convex) Lie group and $\mathfrak{g}$ be its Lie algebra. For a unitary representation $(\pi, \mathcal{H})$ of $G$, we write $\pi^{v}(g):=\pi(g) v$ for its orbit maps and call the representation $(\pi, \mathcal{H})$ of $G$ smooth if the space

$$
\mathcal{H}^{\infty}:=\left\{v \in \mathcal{H}: \pi^{v} \in C^{\infty}(G, \mathcal{H})\right\}
$$

of smooth vectors is dense in $\mathcal{H}$. Then all operators $i \mathrm{~d} \pi(x), x \in \mathfrak{g}$, are essentially selfadjoint and crucial information on their spectrum is contained in the momentum set $I_{\pi}$ of the representation, which is a weak-*-closed convex subset of the topological dual $\mathfrak{g}^{\prime}$. It is defined as the weak-*-closed convex hull of the image of the momentum map on the projective space of $\mathcal{H}^{\infty}$ :

$$
\Phi_{\pi}: \mathbb{P}\left(\mathcal{H}^{\infty}\right) \rightarrow \mathfrak{g}^{\prime} \quad \text { with } \Phi_{\pi}([v])(x)=\frac{1}{i} \frac{\langle\mathrm{d} \pi(x) \cdot v, v\rangle}{\langle v, v\rangle} \quad \text { for }[v]=\mathbb{C} v .
$$

As a weak-*-closed convex subset, $I_{\pi}$ is completely determined by its support functional

$$
s_{\pi}: \mathfrak{g} \rightarrow \mathbb{R} \cup\{\infty\}, \quad s_{\pi}(x)=-\inf \left\langle I_{\pi}, x\right\rangle=\sup (\operatorname{Spec}(i \mathrm{~d} \pi(x))) .
$$

It is now natural to study those representations for which $s_{\pi}$, resp., the set $I_{\pi}$, contains the most significant information, and these are precisely the semibounded ones. As we shall see in Remark 4.8, the geometry of the sets $I_{\pi}$ is closely connected to invariant cones, so that we have to take a closer look at infinite dimensional Lie algebras containing open invariant convex cones $W$ which are pointed in the sense that they do not contain any affine line.

For finite dimensional Lie algebras, there is a well-developed structure theory of invariant convex cones ([36]) and even a characterization of those finite dimensional Lie algebras containing pointed invariant cones ([58,74]; see also [60] for a selfcontained exposition). As the examples described in Sec. 6 show, many key features of the finite dimensional theory survive, but a systematic theory of open invariant cones remains to be developed. A central point of the present note is to exploit properties of open invariant cones for the theory of semibounded representations, in particular to verify that certain unitary representations are semibounded. In Sec. 7, we discuss two aspects of semiboundedness in the representation theory of $C^{*}$-algebras, namely the restrictions of algebra representations to the unitary group $\mathrm{U}(\mathcal{A})$ and covariant representations with respect to a Banach-Lie group acting by automorphisms on $\mathcal{A}$.

Section 8 is devoted to a detailed analysis of invariant convex cones in the Lie algebra $\mathcal{V}\left(\mathbb{S}^{1}\right)$ of smooth vector fields on the circle and its central extension, the 
Virasoro algebra vir. In particular we show that, up to sign, there are only two open invariant cones in $\mathcal{V}\left(\mathbb{S}^{1}\right)$. From this insight we derive that the group $\operatorname{Diff}\left(\mathbb{S}^{1}\right)_{+}$of orientation preserving diffeomorphisms of $\mathbb{S}^{1}$ has no nontrivial semibounded unitary representations, which is derived from the triviality of all unitary highest weight modules ([26]). As one may expect from its importance in mathematical physics, the situation is different for the Virasoro group Vir. For Vir we prove a convexity theorem for adjoint and coadjoint orbits which provides complete information on invariant cones and permits us to determine the momentum sets of the unitary highest weight representations. In particular, we show that these, together with their duals, are precisely the irreducible semibounded unitary representations. Our determination of the momentum sets uses the complex analytic tools from Sec. 5, which lead to a realization in spaces of holomorphic sections on the complex manifold Diff $\left(\mathbb{S}^{1}\right)_{+} / \mathbb{S}^{1}$. This manifold has many interesting realizations. In string theory it occurs as a space of complex structures on the based loop space $C_{*}^{\infty}\left(\mathbb{S}^{1}, \mathbb{R}\right)([13])$, and Kirillov and Yuriev realized it as a space of univalent holomorphic functions on the open complex unit disc $([44,46])$. Its close relative $\operatorname{Diff}\left(\mathbb{S}^{1}\right) / \mathrm{PSL}_{2}(\mathbb{R})$ can be identified with the space of Lorentzian metrics on the one-sheeted hyperboloid (cf. [48]), which leads in particular to an interpretation of the Schwarzian derivative in terms of a conformal factor.

In Secs. 9 and 10 we continue our discussion of important examples with the automorphism groups $\operatorname{Sp}(\mathcal{H})$ of the canonical commutation relations (CCR) and $\mathrm{O}(\mathcal{H})$ of the canonical anticommutation relations $(\mathrm{CAR})$. Geometrically, $\operatorname{Sp}(\mathcal{H})$ is the group of real linear automorphisms of a complex Hilbert space $\mathcal{H}$ preserving the imaginary part $\omega(x, y):=\operatorname{Im}\langle x, y\rangle$ of the scalar product and $\mathrm{O}(\mathcal{H})$ is the group of real linear automorphisms preserving its real part $\beta(x, y):=\operatorname{Re}\langle x, y\rangle$ (cf. [14]). Section 9 is dedicated to the Fock representation of the (CCR). Here we start with the unitary representation $(W, S(\mathcal{H}))$ of the Heisenberg group $\operatorname{Heis}(\mathcal{H})$ on the symmetric/bosonic Fock space $S(\mathcal{H})$. The group $\operatorname{Sp}(\mathcal{H})$ acts naturally by automorphisms $\alpha_{g}$ on $\operatorname{Heis}(\mathcal{H})$ and $W \circ \alpha_{g}$ is equivalent to $W$ if and only if $g$ belongs to the restricted symplectic group $\operatorname{Sp}_{\text {res }}(\mathcal{H})$, i.e. its antilinear part $g_{2}$ is Hilbert-Schmidt. Since the Fock representation of $\operatorname{Heis}(\mathcal{H})$ is irreducible, this leads to a projective representation of $\operatorname{Sp}_{\text {res }}(\mathcal{H})$ on $S(\mathcal{H})$. We write $\widehat{\operatorname{Sp}}_{\text {res }}(\mathcal{H})$ for the corresponding central $\mathbb{T}$-extension; the metaplectic group $([91,96]$, [88, Sec. 5]). Using a quite general smoothness criterion (Theorem A.4), we show that this group carries a natural Banach-Lie group structure and that its canonical unitary representation on $S(\mathcal{H})$ (the metaplectic representation) is smooth. From an explicit formula for the corresponding Lie algebra cocycle, we derive a natural presentation of this group as a quotient of a semidirect product. We further show that the metaplectic representation is semibounded and determine the cone of semibounded elements. The combined representation of the semidirect product $\operatorname{Heis}(\mathcal{H}) \rtimes \widehat{\operatorname{Sp}}_{\text {res }}(\mathcal{H})$ is also semibounded and irreducible, and, using the tools from Sec. 5, we show that its momentum set is the closed convex hull of a single coadjoint orbit. 
In Sec. 10 we then turn to the (CAR), for which we consider the representation on the fermionic Fock space $\Lambda(\mathcal{H})$. Here we likewise obtain a projective representation of the restricted orthogonal group $\mathrm{O}_{\text {res }}(\mathcal{H})([97])$. With completely analogous arguments we then show that the corresponding central extension $\widehat{\mathrm{O}}_{\mathrm{res}}(\mathcal{H})$ is a Lie group, the metagonal group, whose representation on $\Lambda(\mathcal{H})$ (the spin representation) is smooth and semibounded. We also prove that the momentum set of the even spin representation is the weak-*-closed convex hull of a single coadjoint orbit. Our discussion of the bosonic and fermionic Fock representations are very much inspired by the construction of the metaplectic representation in [103] and the presentation in [77]. For finer results on cocycles and connections to physics we refer to $[49,84,88]$. For a more detailed discussion of the metaplectic and the metagonal group, see [104] and [63, Sec. IV.2].

The example of the orthogonal group $\mathrm{O}_{\text {res }}(\mathcal{H})$ is particularly instructive because it shows very naturally how semibounded representations enter the scene for infinite dimensional analogs, such as $\mathrm{O}_{\text {res }}(\mathcal{H})$, of compact groups, for which one rather expects to see bounded representations such as the spin representation of $\mathrm{O}_{1}(\mathcal{H})$ (cf. [59]). In many cases, such as for $\mathrm{O}_{1}(\mathcal{H})$, the class of groups with bounded representations is too restrictive to do justice to the underlying geometry. What makes the larger groups more interesting is the rich supply of exterior automorphisms and the existence of nontrivial central extensions encoding relevant geometric information.

As our examples show, the process of second quantization, i.e. passing from a one-particle Hilbert space to a many-particle space, destroys norm continuity for the representation of the automorphism groups. What survives is semiboundedness for the centrally extended groups acting on the many-particle spaces. This is closely related to the fact that Lie groups of non-unitary maps on a Hilbert space $\mathcal{H}$, such as $\operatorname{Sp}_{\text {res }}(\mathcal{H})$ have unitary representations on the corresponding many-particle spaces. In physics language this means that "unphysical symmetries" of the one-particle Hilbert space may lead to symmetries of the many-particle space (cf. [84]). In the context of finite dimensional Lie groups, the analogous phenomenon is that noncompact matrix groups have nontrivial infinite dimensional unitary representations.

We hope that the detailed discussion of three major classes of representations in Secs. 8-10 demonstrate the close interactions between convex geometry, complex analysis and Lie theory in the context of semibounded unitary representations. Presently, this theory is still in its infancy, but a general picture appears to evolve. One major point is that understanding semibounded unitary representations requires a good deal of knowledge on open invariant cones in the corresponding Lie algebra $\mathfrak{g}$ and, what is closely related, information on the set $\mathfrak{g}_{\text {seq }}^{\prime}$ of semi-equicontinuous coadjoint orbits $\mathcal{O}_{\lambda}$, i.e. orbits for which the function $x \mapsto \sup \mathcal{O}_{\lambda}(x)$ is bounded on some non-empty open subset of $\mathfrak{g}$. Note that for any semibounded representation the momentum set $I_{\pi}$ is contained in $\mathfrak{g}_{\text {seq }}^{\prime}$. In many important cases, the methods developed in Sec. 5 provide a complete description of $I_{\pi}$ in terms of generating coadjoint orbits. 
The main new results and aspects presented in this paper are:

- Section 5 provides tools to verify that unitary representations can be realized in spaces of holomorphic sections of line bundles and to calculate their momentum sets. Here Theorem 5.11 is the key tool.

- The smoothness of the action of a Banach-Lie group on the space of smooth vectors proved in [71] is applied in our context in two essential ways. In Sec. 4, it provides a Hamiltonian action on $\mathbb{P}\left(\mathcal{H}^{\infty}\right)$ for a unitary representation of a Banach-Lie group, and in Sec. 7 it relates a $C^{*}$-dynamical system $(\mathcal{A}, H, \alpha)$ and corresponding covariant representations satisfying a spectral condition to semibounded representations of semidirect product Lie groups of the form $\mathrm{U}\left(\mathcal{A}^{\infty}\right) \rtimes H$. This leads in particular to the remarkable conclusions in Theorem 7.7, which are based on elementary properties of invariant cones.

- The analysis of the convexity properties of adjoint and coadjoint orbits of $\mathcal{V}\left(\mathbb{S}^{1}\right)$ and $\mathfrak{v i r}$ is new. It leads in particular to an identification of the class of irreducible semibounded representations with the unitary highest weight representations and their duals (Theorem 8.22).

- The insights that the Fock representations of $\operatorname{Sp}_{\text {res }}(\mathcal{H})$ and $\mathrm{O}_{\text {res }}(\mathcal{H})$ are semibounded seems to be new, and so are the results on adjoint and coadjoint orbits of the corresponding Banach-Lie groups.

For finite dimensional groups, the first systematic investigation of unitary representations $(\pi, \mathcal{H})$ for which the cone $\left\{x \in \mathfrak{g}: s_{\pi}(x) \leq 0\right\}$ is nontrivial for non-compact simple Lie groups (which are necessarily hermitian by [105]) has been undertaken in the pioneering work of G. Olshanski ([76]). Based on the powerful structure theory for invariant cones developed in [36] by Hofmann, Hilgert and Lawson, we were eventually able to develop a general theory for semibounded representations of finite dimensional Lie groups, including a classification and a disintegration theory ([60]). We hope that one can also develop a similarly rich theory of complex semigroups and holomorphic extensions, so that $C^{*}$-algebraic tools become available to deal with direct integrals of semibounded representations. In [67] we undertook some first steps in this direction, including a complete theory for the Abelian case (cf. Theorem 5.2). What is needed here is a good theory of analytic vectors, which becomes a tricky issue for infinite dimensional Lie groups. Up to now, existence of analytic vectors is only known for very special classes of groups such as certain direct limits ([85]) and the canonical commutator relations in Quantum Field Theory $([82,34])$.

If $K$ is a compact simple Lie group and $\mathcal{L}(K):=C^{\infty}\left(\mathbb{S}^{1}, K\right)$ the corresponding loop group, then the group $T_{r}:=\mathbb{T}=\mathbb{R} / \mathbb{Z}$ acts smoothly by rotations on $\mathcal{L}(K)$ and also on its canonical central extension $\widetilde{\mathcal{L}}(K)$ by $\mathbb{T}$, which leads to the "smooth version" $\widehat{\mathcal{L}}(K):=\widetilde{\mathcal{L}}(K) \rtimes \mathbb{T}$ of affine Kac-Moody groups. For these groups positive energy representations are defined by requiring the spectrum of the generator of $T_{r}$ to be bounded below in the representation. Similarly, one defines positive energy representations of the group $\operatorname{Diff}\left(\mathbb{S}^{1}\right)_{+}$of orientation preserving diffeomorphisms 
of the circle. Various aspects of the theory of irreducible positive energy representations were developed in $[88,27,81,100,101]$, but only in $[81$, Secs. 9.3, 11.4; Proposition 11.2.5] one finds some attempts towards a decomposition theory.

Positivity conditions for spectra also play a key role in Segal's concept of physical representations of the full unitary group $\mathrm{U}(\mathcal{H})$ of a Hilbert space $\mathcal{H}$, endowed with the strong operator topology $([89])$. Here the positivity requirements even imply boundedness of the representation, discrete decomposability and even a classification of the irreducible representations.

\section{Semi-Equicontinuous Convex Sets}

Let $E$ be a real locally convex space and $E^{\prime}$ be its topological dual, i.e. the space of continuous linear functionals on $E$. We write $\langle\alpha, v\rangle=\alpha(v)$ for the natural pairing $E^{\prime} \times E \rightarrow \mathbb{R}$ and endow $E^{\prime}$ with the weak-*-topology, i.e. the coarsest topology for which all linear maps

$$
\eta_{v}: E^{\prime} \rightarrow \mathbb{R}, \quad \eta_{v}(\alpha):=\alpha(v)
$$

are continuous. For a subset $X \subseteq E^{\prime}$, the set

$$
B(X):=\{v \in E: \inf \langle X, v\rangle>-\infty\}
$$

is a convex cone which coincides with the domain of the support function

$$
s_{X}: E \rightarrow \mathbb{R} \cup\{\infty\}, \quad s_{X}(v):=-\inf \langle X, v\rangle=\sup \langle X,-v\rangle
$$

of $X$ in the sense that $B(X)=s_{X}^{-1}(\mathbb{R})$. As a sup of a family of continuous linear functionals, the function $s_{X}$ is convex, lower semicontinuous and positively homogeneous.

Definition 2.1. We call a subset $X \subseteq E^{\prime}$ semi-equicontinuous if $s_{X}$ is bounded on some non-empty open subset of $E$ (cf. [68]). This implies in particular that the cone $B(X)$ has interior points and even that $s_{X}$ is continuous on $B(X)^{0}([67$, Proposition 6.8]).

If the space $E$ is barreled, which includes in particular Banach and Fréchet spaces, we have the following handy criterion for semi-equicontinuity. We only have to apply [67, Theorem 6.10] to the lower semicontinuous function $s_{X}$.

Proposition 2.2. If $E$ is barreled, then $X \subseteq E^{\prime}$ is semi-equicontinuous if and only if $B(X)$ has interior points.

Remark 2.3. (a) The notion of semi-equicontinuity generalizes the notion of equicontinuity, which is equivalent to $s_{X}$ being bounded on some 0-neighborhood of $E$. In fact, the boundedness of $s_{X}$ on some symmetric 0-neighborhood $U=-U$ means that there exists a $C>0$ with $s_{X}( \pm v) \leq C$ for $v \in U$. This is equivalent to $|\alpha(v)| \leq C$ for $v \in U, \alpha \in X$, which means that $X$ is equicontinuous. 
(b) If $Y:=\overline{\operatorname{conv}}(X)$ denotes the weak-*-closed convex hull of $X$, then $s_{X}=$ $s_{Y}$, and, using the Hahn-Banach Separation Theorem, $Y$ can be reconstructed from $s_{Y}$ by

$$
Y=\left\{\alpha \in E^{\prime}:(\forall v \in B(Y)) \alpha(v) \geq \inf \langle Y, v\rangle=-s_{Y}(v)\right\}
$$

If, in addition, the interior $B(Y)^{0}$ is non-empty, then we even have

$$
Y=\left\{\alpha \in E^{\prime}:\left(\forall v \in B(Y)^{0}\right) \alpha(v) \geq \inf \langle Y, v\rangle=-s_{Y}(v)\right\}
$$

([67, Proposition 6.4]).

Definition 2.4. (a) For a convex subset $C \subseteq E$ we put

$$
\lim (C):=\{x \in E: C+x \subseteq C\}
$$

and

$$
H(C):=\lim (C) \cap-\lim (C):=\{x \in E: C+x=C\} .
$$

Then $\lim (C)$ is a convex cone and $H(C)$ a linear subspace of $E$.

(b) A convex cone $W \subseteq E$ is called pointed if $H(W)=\{0\}$.

(c) For a subset $C \subseteq E$,

$$
C^{\star}:=\left\{\alpha \in E^{\prime}: \alpha(C) \subseteq \mathbb{R}_{+}\right\}
$$

is called the dual cone and for a subset $X \subseteq E^{\prime}$, we define the dual cone by

$$
X^{\star}:=\left\{v \in E:\langle X, v\rangle \subseteq \mathbb{R}_{+}\right\} .
$$

Examples 2.5. (a) If $E$ is a Banach space, then the unit ball

$$
X:=\left\{\alpha \in E^{\prime}:\|\alpha\| \leq 1\right\}
$$

in $E^{\prime}$ is equicontinuous because the Hahn-Banach Theorems imply that $s_{X}(v)=$ $\|v\|$ for $v \in E$.

(b) If $\emptyset \neq \Omega \subseteq E$ is an open convex cone, then its dual cone $\Omega^{\star}$ is semiequicontinuous because we have $s_{\Omega^{\star}}=0$ on $\bar{\Omega}=\left(\Omega^{\star}\right)^{\star}$ and $s_{\Omega^{\star}}=\infty$ on the complement of this closed cone.

We have just seen that open convex cones lead to semi-equicontinuous sets. There is also a partial converse:

Remark 2.6. Let $X \subseteq E^{\prime}$ be a semi-equicontinuous set and $\widetilde{E}:=E \oplus \mathbb{R}$. For the set

$$
\widetilde{X}:=X \times\{1\} \subseteq \widetilde{E}^{\prime} \quad \text { we then have } s_{\widetilde{X}}(v, t):=t+s_{X}(v),
$$

so that the boundedness of $s_{X}$ on some non-empty open subset of $E$ implies that the interior of the dual cone $\widetilde{X}^{\star} \subseteq \widetilde{E}$ is non-empty. In view of Example 2.5(b), this means that $X$ is semi-equicontinuous if and only if it can be embedded into the dual of some open convex cone in $\widetilde{E}$. 
The following observation shows that semi-equicontinuous convex sets share many important properties with compact convex sets (cf. [67, Proposition 6.13]):

Proposition 2.7. Let $X \subseteq E^{\prime}$ be a non-empty weak-*-closed convex subset and $v \in E$ such that the support function $s_{X}$ is bounded above on some neighborhood of $v$. Then $X$ is weak-*-locally compact, the function

$$
\eta_{v}: X \rightarrow \mathbb{R}, \quad \eta_{v}(\alpha):=\alpha(v)
$$

is proper, and there exists an extreme point $\alpha \in X$ with $\alpha(v)=\min \langle X, v\rangle$.

We conclude this section with some elementary properties of convex subsets of locally convex spaces.

The following lemma ([12, Corollary II.2.6.1]) is often useful:

Lemma 2.8. For a convex subset $C$ of a locally convex space $E$ the following assertions hold:

(i) $C^{0}$ and $\bar{C}$ are convex.

(ii) $\bar{C}^{0}=C^{0}$ and if $C^{0} \neq \emptyset$, then $\overline{C^{0}}=\bar{C}$.

Lemma 2.9. If $\emptyset \neq C \subseteq E$ is an open or closed convex subset, then the following assertions hold:

(i) $\lim (C)=\lim (\bar{C})$ is a closed convex cone.

(ii) $\lim (C)=\left\{v \in E: v=\lim _{n \rightarrow \infty} t_{n} c_{n}, c_{n} \in C, t_{n} \rightarrow 0, t_{n} \geq 0\right\}$. If $t_{j} c_{j} \rightarrow v$ holds for a net with $t_{j} \geq 0$ and $t_{j} \rightarrow 0$ and $c_{j} \in C$, then also $v \in \lim (C)$.

(iii) If $c \in C$ and $d \in E$ satisfy $c+\mathbb{N} d \subseteq C$, then $d \in \lim (C)$.

(iv) $c+\mathbb{R} d \subseteq C$ implies $d \in H(C)$. In particular, $H(C)=\{0\}$ if and only if $C$ contains no affine lines.

(v) $H(C)$ is closed and the subset $C / H(C) \subseteq E / H(C)$ contains no affine lines.

(vi) $B(C)^{\star}=\lim (C)$ and $B(C)^{\perp}=H(C)$.

Proof. (cf. [67, Proposition 6.1]) (i) If $C$ is open, then $C=(\bar{C})^{0}$ by Lemma 2.8, and thus $x+C \subseteq C$ is equivalent to $x+\bar{C} \subseteq \bar{C}$. In particular, $\lim (C)$ is closed.

(ii) If $c \in C$ and $x \in \lim (C)$, then $c+n x \in C$ for $n \in \mathbb{N}$ and $\frac{1}{n}(c+n x) \rightarrow x$. If, conversely, $x=\lim t_{j} c_{j}$ with $t_{j} \rightarrow 0, t_{j} \geq 0, c_{j} \in C$, and $c \in \bar{C}$, then $\left(1-t_{j}\right) c+$ $t_{j} c_{j} \rightarrow c+x \in \bar{C}$ implies that $\bar{C}+x \subseteq \bar{C}$, i.e. $x \in \lim (\bar{C})=\lim (C)$.

(iii) In view of $\frac{1}{n}(c+n d) \rightarrow d$, (ii) implies $d \in \lim (C)$.

(iv) immediately follows from (iii).

(v) The closedness of $H(C)=\lim (C) \cap-\lim (C)$ follows from (i). Therefore the quotient topology on $E / H(C)$ is Hausdorff, so that the quotient topology turns $E / H(C)$ into a locally convex space. Let $q: E \rightarrow E / H(C)$ denote the quotient map. If $y+\mathbb{R} d \subseteq q(C)=C / H(C)$ is an affine line and $y=q(x), d=q(c)$, then $x+\mathbb{R} c \subseteq q^{-1}(C / H(C))=C$ implies $c \in H(C)$ by (iv), which leads to $d=q(c)=0$. Hence $C / H(C)$ contains no affine lines. 
(vi) From

$$
\bar{C}=\{v \in E:(\forall \alpha \in B(C)) \alpha(v) \geq \inf \alpha(C)\}
$$

(a consequence of the Hahn-Banach Separation Theorem), we derive that $B(C)^{\star} \subseteq$ $\lim (\bar{C})=\lim (C)$. Conversely, $\lim (C) \subseteq B(C)^{\star}$ follows immediately from $c+$ $\lim (C) \subseteq C$ for each $c \in C$.

Remark 2.10. If $X \subseteq E^{\prime}$ is semi-equicontinuous, then $B(X)$ has interior points, so that $H(X) \subseteq B(X)^{\perp}=\{0\}$ (Lemma 2.9(vi)) implies $H(X)=\{0\}$, i.e. $X$ contains no affine lines. If, conversely, $H(X)=\{0\}$ and $\operatorname{dim} V<\infty$, then it follows from [60, Proposition V.1.15] that $X$ is semi-equicontinuous. Therefore closed convex subsets of finite dimensional vector spaces are semi-equicontinuous if and only if they contain no affine lines.

For later applications, we record the following fact on fixed point projections for actions of compact groups.

Proposition 2.11. Let $K$ be a compact group acting continuously on the complete locally convex space $E$ by the representation $\pi: K \rightarrow \mathrm{GL}(E)$.

(a) If $\Omega \subseteq E$ is an open or closed $K$-invariant convex subset, then $\Omega$ is invariant under the fixed point projection

$$
p(v):=\int_{K} \pi(k) v d \mu_{K}(k),
$$

where $\mu_{K}$ is a normalized Haar measure on $K$.

(b) If $C \subseteq E^{\prime}$ is a weak-*-closed convex $K$-invariant subset, then $C$ is invariant under the adjoint

$$
p^{\prime}(\lambda) v:=\lambda(p(v))=\int_{K} \lambda(\pi(k) v) d \mu_{K}(k)
$$

of $p$.

Proof. (a) The existence of the integrals defining the projection $p$ follows from the completeness of $E$ (cf. [39, Proposition 3.30]). Let $\lambda \in B(\Omega) \subseteq E^{\prime}$ be a continuous linear functional bounded below on $\Omega$.

If $\Omega$ is open, we then have $\lambda(\pi(k) v)>\inf \lambda(\Omega)$ for every $k \in K$, so that

$$
\lambda(p(v))=\int_{K} \lambda(\pi(k) v) d \mu_{K}(k)>\inf \lambda(\Omega) .
$$

In view of the Hahn-Banach Separation Theorem, this implies that $p(v) \in \bar{\Omega}$ (Remark 2.3(b)). If $p(v) \in \partial \Omega$, then [12, Proposition II.5.2.3] implies the existence of $\lambda \in B(\Omega)=B(\bar{\Omega})$ with $\lambda(p(v))=\min \lambda(\bar{\Omega})=\inf \lambda(\Omega)$, a contradiction. Therefore $p(v) \in \bar{\Omega}^{0}=\Omega$ (cf. Lemma 2.8).

If $\Omega$ is closed, then the preceding argument implies $\lambda(p(v)) \geq \inf \lambda(\Omega)$, and hence that $p(v) \in \Omega$ by the Separation Theorem. 
(b) Now let $C \subseteq E^{\prime}$ be weak-*-closed and $G$-invariant. For each $v \in E$ and $\lambda \in C$, we then have

$$
p^{\prime}(\lambda)(v)=\lambda(p(v))=\int_{K} \lambda(\pi(k) v) d \mu_{K}(k) \geq \inf \langle C, v\rangle,
$$

so that the Hahn-Banach Separation Theorem shows that $p^{\prime}(\lambda) \in C$.

\section{Infinite Dimensional Lie Groups}

In this section we provide the definition of a locally convex Lie group and present several key examples that will show up later in our discussion of semibounded representations.

Definition 3.1. (a) Let $E$ and $F$ be locally convex spaces, $U \subseteq E$ open and $f: U \rightarrow F$ a map. Then the derivative of $f$ at $x$ in the direction $h$ is defined as

$$
\mathrm{d} f(x)(h):=\left(\partial_{h} f\right)(x):=\left.\frac{d}{d t}\right|_{t=0} f(x+t h)=\lim _{t \rightarrow 0} \frac{1}{t}(f(x+t h)-f(x))
$$

whenever it exists. The function $f$ is called differentiable at $x$ if $\mathrm{d} f(x)(h)$ exists for all $h \in E$. It is called continuously differentiable, if it is differentiable at all points of $U$ and

$$
\mathrm{d} f: U \times E \rightarrow F, \quad(x, h) \mapsto \mathrm{d} f(x)(h)
$$

is a continuous map. Note that this implies that the maps d $f(x)$ are linear (cf. [25, Lemma 2.2.14]). The map $f$ is called a $C^{k}$-map, $k \in \mathbb{N} \cup\{\infty\}$, if it is continuous, the iterated directional derivatives

$$
\mathrm{d}^{j} f(x)\left(h_{1}, \ldots, h_{j}\right):=\left(\partial_{h_{j}} \cdots \partial_{h_{1}} f\right)(x)
$$

exist for all integers $j \leq k, x \in U$ and $h_{1}, \ldots, h_{j} \in E$, and all maps d $\mathrm{d}^{j} f: U \times E^{j} \rightarrow F$ are continuous. As usual, $C^{\infty}$-maps are called smooth.

(b) If $E$ and $F$ are complex locally convex spaces, then a map $f$ is called complex analytic if it is continuous and for each $x \in U$ there exists a 0-neighborhood $V$ with $x+V \subseteq U$ and continuous homogeneous polynomials $\beta_{k}: E \rightarrow F$ of degree $k$ such that for each $h \in V$ we have

$$
f(x+h)=\sum_{k=0}^{\infty} \beta_{k}(h),
$$

as a pointwise limit ([9]). The map $f$ is called holomorphic if it is $C^{1}$ and for each $x \in U$ the map d $f(x): E \rightarrow F$ is complex linear (cf. [57, p. 1027]). If $F$ is sequentially complete, then $f$ is holomorphic if and only if it is complex analytic (cf. [24], [9, Theorems 3.1 and 6.4]).

(c) If $E$ and $F$ are real locally convex spaces, then we call $f$ real analytic, resp., $C^{\omega}$, if for each point $x \in U$ there exists an open neighborhood $V \subseteq E_{\mathbb{C}}$ and a holomorphic map $f_{\mathbb{C}}: V \rightarrow F_{\mathbb{C}}$ with $\left.f_{\mathbb{C}}\right|_{U \cap V}=\left.f\right|_{U \cap V}$ (cf. [57]). The advantage of this definition, which differs from the one in [9], is that it works nicely for non-complete 
spaces, any analytic map is smooth, and the corresponding chain rule holds without any condition on the underlying spaces, which is the key to the definition of analytic manifolds (see [24] for details).

Once one has introduced the concept of a smooth function between open subsets of locally convex spaces, it is clear how to define a locally convex smooth manifold. A (locally convex) Lie group $G$ is a group equipped with a smooth manifold structure modeled on a locally convex space for which the group multiplication and the inversion are smooth maps. We write $\mathbf{1} \in G$ for the identity element and $\lambda_{g}(x)=g x$, resp., $\rho_{g}(x)=x g$ for the left, resp., right multiplication on $G$. Then each $x \in T_{\mathbf{1}}(G)$ corresponds to a unique left invariant vector field $x_{l}$ with $x_{l}(g):=T_{\mathbf{1}}\left(\lambda_{g}\right) x, g \in G$. The space of left invariant vector fields is closed under the Lie bracket of vector fields, hence inherits a Lie algebra structure. In this sense we obtain on $\mathfrak{g}:=T_{\mathbf{1}}(G)$ a continuous Lie bracket which is uniquely determined by $[x, y]_{l}=\left[x_{l}, y_{l}\right]$ for $x, y \in \mathfrak{g}$. We shall also use the functorial notation $\mathbf{L}(G):=(\mathfrak{g},[\cdot, \cdot])$ for the Lie algebra of $G$ and, accordingly, $\mathbf{L}(\varphi)=T_{\mathbf{1}}(\varphi): \mathbf{L}\left(G_{1}\right) \rightarrow \mathbf{L}\left(G_{2}\right)$ for the Lie algebra morphism associated to a morphism $\varphi: G_{1} \rightarrow G_{2}$ of Lie groups. Then $\mathbf{L}$ defines a functor from the category of locally convex Lie groups to the category of locally convex topological Lie algebras. The adjoint action of $G$ on $\mathbf{L}(G)$ is defined by $\operatorname{Ad}(g):=$ $\mathbf{L}\left(c_{g}\right)$, where $c_{g}(x)=g x g^{-1}$. This action is smooth and each $\operatorname{Ad}(g)$ is a topological isomorphism of $\mathbf{L}(G)$. The coadjoint action on the topological dual space $\mathbf{L}(G)^{\prime}$ is defined by

$$
\operatorname{Ad}^{*}(g) \alpha:=\alpha \circ \operatorname{Ad}(g)^{-1}
$$

and the maps $\operatorname{Ad}^{*}(g)$ are continuous with respect to the weak-*-topology on $\mathbf{L}(G)^{\prime}$, but in general the coadjoint action of $G$ is not continuous with respect to this topology. If $\mathfrak{g}$ is a Fréchet, resp., a Banach space, then $G$ is called a Fréchet-, resp., a Banach-Lie group.

A smooth map $\exp _{G}: \mathbf{L}(G) \rightarrow G$ is called an exponential function if each curve $\gamma_{x}(t):=\exp _{G}(t x)$ is a one-parameter group with $\gamma_{x}^{\prime}(0)=x$. The Lie group $G$ is said to be locally exponential if it has an exponential function for which there is an open 0-neighborhood $U$ in $\mathbf{L}(G)$ mapped diffeomorphically by $\exp _{G}$ onto an open subset of $G$. All Banach-Lie groups are locally exponential ([66, Proposition IV.1.2]). Not every infinite dimensional Lie group has an exponential function ([66, Ex. II.5.5]), but exponential functions are unique whenever they exist.

In the context of unitary representation theory, the exponential function permits us to associate to each element $x$ of the Lie algebra a unitary one-parameter group $\pi_{x}(t):=\pi\left(\exp _{G} t x\right)$. We therefore assume in the following that $G$ has an exponential function.

Examples 3.2. Here are some important examples of infinite dimensional Lie groups that we shall encounter below.

(a) (Unitary groups) If $\mathcal{A}$ is a unital $C^{*}$-algebra, then its unitary group

$$
\mathrm{U}(\mathcal{A}):=\left\{g \in \mathcal{A}: g^{*} g=g g^{*}=\mathbf{1}\right\}
$$


is a Banach-Lie group with Lie algebra

$$
\mathfrak{u}(\mathcal{A})=\left\{x \in \mathcal{A}: x^{*}=-x\right\} .
$$

In particular, the unitary group $\mathrm{U}(\mathcal{H})=\mathrm{U}(B(\mathcal{H}))$ of a complex Hilbert space $\mathcal{H}$ is of this form, and we write $\mathfrak{u}(\mathcal{H})$ for its Lie algebra.

As we shall see below (Definition 7.4), in some situations one is forced to consider more general classes of algebras: A locally convex topological unital algebra $\mathcal{A}$ is called a continuous inverse algebra if its unit group $\mathcal{A}^{\times}$is open and the inversion map $a \mapsto a^{-1}$ is continuous. This condition already implies that $\mathcal{A}^{\times}$, endowed with the canonical manifold structure as an open subset, is a Lie group (cf. [24]). If, in addition, $\mathcal{A}$ is a complex algebra and $*$ a continuous algebra involution, then

$$
\mathrm{U}(\mathcal{A}):=\left\{g \in \mathcal{A}: g^{*} g=g g^{*}=\mathbf{1}\right\}
$$

is a closed subgroup, and even a submanifold, as is easily seen with the Cayley transform $c(x)=(\mathbf{1}-x)(\mathbf{1}+x)^{-1}$. It defines an involutive diffeomorphism of some open neighborhood of $\mathbf{1}$ onto some open neighborhood of 0 satisfying $c(x)^{*}=c\left(x^{*}\right)$ and $c\left(x^{-1}\right)=-c(x)$. In particular, $c(g)$ is skew-hermitian if and only if $g$ is unitary, and since $\mathrm{U}(\mathcal{A})$ is a subgroup of $\mathcal{A}^{\times}$, this argument shows that it actually is a Lie subgroup.

(b) (Schatten class groups) If $\mathcal{H}$ is a real or complex Hilbert space and $B_{p}(\mathcal{H})$ denotes the $p$-Schatten ideal $(p \geq 1)$ with the norm $\|A\|_{p}:=\operatorname{tr}\left(\left(A^{*} A\right)^{p / 2}\right)^{1 / p}$, then

$$
\mathrm{U}_{p}(\mathcal{H}):=\mathrm{U}(\mathcal{H}) \cap\left(\mathbf{1}+B_{p}(\mathcal{H})\right)
$$

is a Banach-Lie group with Lie algebra

$$
\mathfrak{u}_{p}(\mathcal{H}):=\mathfrak{u}(\mathcal{H}) \cap B_{p}(\mathcal{H})
$$

(cf. $[56,65])$.

(c) (Restricted groups) If $P$ is an orthogonal projection on $\mathcal{H}$ and $G \subseteq \mathrm{GL}(\mathcal{H})$ a subgroup, then we call

$$
G_{\text {res }}:=\left\{g \in G:[g, P] \in B_{2}(\mathcal{H})\right\}
$$

the corresponding restricted group. Using the fact that $B_{2}(\mathcal{H})$ is invariant under left and right multiplication with elements of $G$, it is easy to see that this is indeed a subgroup, and in many cases it carries a natural Banach-Lie group structure. Writing $\mathcal{H}=\operatorname{im}(P) \oplus \operatorname{ker}(P)$ and, accordingly, operators on $\mathcal{H}$ as $(2 \times 2)$-matrices, then

$$
g=\left(\begin{array}{ll}
a & b \\
c & d
\end{array}\right) \in G_{\mathrm{res}} \Leftrightarrow b, c \in B_{2}(\mathcal{H}) .
$$

In particular, we have the restricted unitary group

$$
\mathrm{U}_{\text {res }}(\mathcal{H}, P):=\left\{g \in \mathrm{U}(\mathcal{H}):[g, P] \in B_{2}(\mathcal{H})\right\} .
$$

(d) If $\mathcal{H}$ is a complex Hilbert space, then the scalar product $\langle\cdot, \cdot\rangle$ (always assumed to be linear in the first component), defines two real bilinear forms

$$
\beta(x, y):=\operatorname{Re}\langle x, y\rangle \text { and } \omega(x, y):=\operatorname{Im}\langle x, y\rangle=\operatorname{Re}\langle x, I y\rangle,
$$


where $\beta$ is symmetric and $\omega$ is skew-symmetric. Writing $\mathcal{H}_{\mathbb{R}}$ for the underlying real Banach space, we thus obtain the symplectic group

$$
\begin{aligned}
\operatorname{Sp}(\mathcal{H}) & :=\operatorname{Sp}\left(\mathcal{H}_{\mathbb{R}}, \omega\right):=\left\{g \in \operatorname{GL}\left(\mathcal{H}_{\mathbb{R}}\right):\left(\forall v, w \in \mathcal{H}_{\mathbb{R}}\right) \omega(g v, g w)=\omega(v, w)\right\} \\
& =\left\{g \in \operatorname{GL}\left(\mathcal{H}_{\mathbb{R}}\right): g^{\top} I g=I\right\}
\end{aligned}
$$

and the orthogonal group

$$
\begin{aligned}
\mathrm{O}(\mathcal{H}) & :=\mathrm{O}\left(\mathcal{H}_{\mathbb{R}}, \beta\right):=\left\{g \in \mathrm{GL}\left(\mathcal{H}_{\mathbb{R}}\right):\left(\forall v, w \in \mathcal{H}_{\mathbb{R}}\right) \beta(g v, g w)=\beta(v, w)\right\} \\
& =\left\{g \in \mathrm{GL}\left(\mathcal{H}_{\mathbb{R}}\right): g^{\top} g=\mathbf{1}\right\} .
\end{aligned}
$$

There are two important variants of these groups, namely the Hilbert-Lie groups

$$
\operatorname{Sp}_{2}(\mathcal{H}):=\operatorname{Sp}(\mathcal{H}) \cap\left(\mathbf{1}+B_{2}\left(\mathcal{H}_{\mathbb{R}}\right)\right), \quad \mathrm{O}_{2}(\mathcal{H}):=\mathrm{O}(\mathcal{H}) \cap\left(\mathbf{1}+B_{2}\left(\mathcal{H}_{\mathbb{R}}\right)\right)
$$

and the restricted groups

$$
\operatorname{Sp}_{\text {res }}(\mathcal{H}):=\left\{g \in \operatorname{Sp}(\mathcal{H}):[g, I] \in B_{2}\left(\mathcal{H}_{\mathbb{R}}\right)\right\}
$$

and

$$
\mathrm{O}_{\mathrm{res}}(\mathcal{H}):=\left\{g \in \mathrm{O}(\mathcal{H}):[g, I] \in B_{2}\left(\mathcal{H}_{\mathbb{R}}\right)\right\}
$$

where $I v:=i v$ denotes the complex structure on $\mathcal{H}_{\mathbb{R}}$ defining the complex Hilbert space $\mathcal{H}$.

The groups $\operatorname{Sp}\left(\mathcal{H}_{\mathbb{R}}, \omega\right)$ and $\mathrm{O}\left(\mathcal{H}_{\mathbb{R}}, \beta\right)$ play a key role in Quantum Field Theory as the automorphism groups of the canonical commutation relations (CCR) and the canonical anticommutation relations (CAR) (cf. [14]). However, only the corresponding restricted groups, resp., their central extensions, have corresponding unitary representations (cf. Secs. 9 and 10).

(e) The group $\operatorname{Diff}(M)^{\text {op }}$ of diffeomorphisms of a compact manifold $M$ is a Lie group with respect to the group structure defined by $\varphi \cdot \psi:=\psi \circ \varphi$. Its Lie algebra is the space $\mathcal{V}(M)$ of smooth vector fields on $M$ with respect to the natural Lie bracket. The use of the opposite group simplifies many formulas and minimizes the number of negative signs. In particular, it implies that the exponential function is given by the time 1-flow and not by its inverse. As we shall see below, this convention also leads to simpler formulas because the action of Diff $(M)^{\text {op }}$ by pullbacks is a left action.

(f) If $K$ is a Lie group and $M$ is a compact manifold, then the space $C^{\infty}(M, K)$ of smooth maps is a Lie group with Lie algebra $C^{\infty}(M, \mathfrak{k})$, where $\mathfrak{k}$ is the Lie algebra of $K$.

(g) A domain $\mathcal{D}$ in the complex Banach space $V$ is said to be symmetric if there exists for each point $x \in \mathcal{D}$ a biholomorphic involution $s_{x} \in \operatorname{Aut}(\mathcal{D})$ for which $x$ is an isolated fixed point, or, equivalently $T_{x}\left(s_{x}\right)=-\mathrm{id}$. It is called a symmetric Hilbert domain if, in addition, $V$ is a complex Hilbert space. Then the group $\operatorname{Aut}(\mathcal{D})$ of biholomorphic automorphisms of $\mathcal{D}$ carries a natural Banach-Lie group structure (cf. [43, Sec. V], [102], [61, Theorem V.11]). 
Here is a typical example. If $\mathcal{H}_{ \pm}$are two complex Hilbert spaces and $B_{2}\left(\mathcal{H}_{+}, \mathcal{H}_{-}\right)$ is the Hilbert space of Hilbert-Schmidt operators from $\mathcal{H}_{+}$to $\mathcal{H}_{-}$, then

$$
\mathcal{D}:=\left\{z \in B_{2}\left(\mathcal{H}_{+}, \mathcal{H}_{-}\right):\|z\|<1\right\}
$$

is a symmetric Hilbert domain, where $\|\cdot\|$ denotes the operator norm, which is smaller than the Hilbert-Schmidt norm $\|\cdot\|_{2}$. In particular, $\mathcal{D}$ is unbounded if both spaces are infinite dimensional. On the Hilbert space $\mathcal{K}:=\mathcal{H}_{-} \oplus \mathcal{H}_{+}$we define a hermitian form by $\gamma\left((v, w),\left(v^{\prime}, w^{\prime}\right)\right):=\left\langle v, v^{\prime}\right\rangle-\left\langle w, w^{\prime}\right\rangle$ and write

$$
\mathrm{U}\left(\mathcal{H}_{+}, \mathcal{H}_{-}\right):=\{g \in \operatorname{GL}(\mathcal{K}):(\forall x, y \in \mathcal{K}) \gamma(g x, g y)=\gamma(x, y)\}
$$

for the corresponding pseudo-unitary group. From the projection $P\left(v_{-}, v_{+}\right):=$ $\left(v_{-}, 0\right)$ we now obtain a restricted pseudo-unitary group

$$
\mathrm{U}_{\text {res }}\left(\mathcal{H}_{+}, \mathcal{H}_{-}\right)=\left\{g \in \mathrm{U}\left(\mathcal{H}_{+}, \mathcal{H}_{-}\right):\|[g, P]\|_{2}<\infty\right\}
$$

(cf. (c) above), and this group acts on $\mathcal{D}$ by

$$
g \cdot z=\left(\begin{array}{ll}
a & b \\
c & d
\end{array}\right) \cdot z:=(a z+b)(c z+d)^{-1} .
$$

The subgroup $\mathbb{T} \mathbf{1}$ of $\mathrm{U}_{\text {res }}\left(\mathcal{H}_{+}, \mathcal{H}_{-}\right)$acts trivially, and we thus obtain an isomorphism

$$
\mathrm{U}_{\mathrm{res}}\left(\mathcal{H}_{+}, \mathcal{H}_{-}\right) / \mathbb{T} 1 \cong \operatorname{Aut}(\mathcal{D})_{0}
$$

(combine [43, Sec. 5] on the automorphisms of the completion with respect to the operator norm with the extension result in [61, Theorem V.11]).

(h) In (g), the case $\mathcal{H}=\mathcal{H}_{+}=\mathcal{H}_{-}$is of particular interest because it leads to two other series of irreducible symmetric Hilbert domains generalizing the operator balls in symmetric, resp., skew-symmetric matrices. Let $\sigma$ be an antilinear isometric involution on $\mathcal{H}$ and define $x^{\top}:=\sigma x^{*} \sigma$ for $x \in B(\mathcal{H})$. Then the map $\iota: \mathcal{H} \rightarrow \mathcal{K}, v \mapsto \frac{1}{\sqrt{2}}(v, \sigma v)$ is an antilinear isometric embedding whose image is an orthogonal direct $\operatorname{sum} \mathcal{K}=\iota(\mathcal{H}) \oplus i \iota(\mathcal{H})$, so that $\mathcal{K} \cong\left(\mathcal{H}_{\mathbb{R}}\right)_{\mathbb{C}}$ as Hilbert spaces, where the complex conjugation on $\mathcal{K}$ is given by $\tau(v, w)=(\sigma w, \sigma v)$. By complex linear extension, we thus obtain an embedding $\gamma: \operatorname{Sp}(\mathcal{H}) \hookrightarrow \operatorname{GL}(\mathcal{K})$, whose image preserves the real subspace $\iota(\mathcal{H})$ and the complex bilinear skew-symmetric form $\omega_{\mathbb{C}}$ obtained by complex bilinear extension of $\omega(x, y)=\operatorname{Im}\langle x, y\rangle$. Since the complex bilinear form

$$
\omega_{\mathbb{C}}\left((x, y),\left(x^{\prime}, y^{\prime}\right)\right):=\left\langle x, \sigma y^{\prime}\right\rangle-\left\langle x^{\prime}, \sigma y\right\rangle
$$

on $\mathcal{K}$ satisfies

$$
\omega_{\mathbb{C}}((x, \sigma x),(y, \sigma y))=\langle x, y\rangle-\langle y, x\rangle=2 i \operatorname{Im}\langle x, y\rangle=2 i \omega(x, y),
$$

the subgroup $\gamma(\operatorname{Sp}(\mathcal{H}))$ is contained in $\operatorname{Sp}\left(\mathcal{K}, \omega_{\mathbb{C}}\right)$. From the fact that it also commutes with $\tau$ one easily derives that it also preserves the canonical hermitian form 
$\gamma\left((x, y),\left(x^{\prime}, y^{\prime}\right)\right)=\left\langle x, x^{\prime}\right\rangle-\left\langle y, y^{\prime}\right\rangle$, and this leads to an isomorphism

$$
\operatorname{Sp}(\mathcal{H}) \cong \gamma(\operatorname{Sp}(\mathcal{H}))=\mathrm{U}(\mathcal{H}, \mathcal{H}) \cap \operatorname{Sp}\left(\mathcal{K}, \omega_{\mathbb{C}}\right)
$$

(cf. [63, Remark I.2], [72, Sec. IV]). This in turn leads to isomorphisms

$$
\operatorname{Sp}_{\text {res }}(\mathcal{H}) \cong \mathrm{U}_{\text {res }}(\mathcal{H}, \mathcal{H}) \cap \operatorname{Sp}\left(\mathcal{K}, \omega_{\mathbb{C}}\right)
$$

and

$$
\mathfrak{s p}_{\text {res }}(\mathcal{H}) \cong\left\{\left(\begin{array}{cc}
a & b \\
b^{*} & -a^{\top}
\end{array}\right): a \in \mathfrak{u}(\mathcal{H}), b=b^{\top} \in B_{2}(\mathcal{H})\right\} .
$$

From that one further derives that $\operatorname{Sp}_{\text {res }}(\mathcal{H})$ acts by holomorphic automorphisms on the symmetric Hilbert domain

$$
\mathcal{D}_{s}:=\left\{z \in B_{2}(\mathcal{H}): z^{\top}=z,\|z\|<1\right\}
$$

by fractional linear transformations, and we thus obtain isomorphisms

$$
\operatorname{Sp}_{\text {res }}(\mathcal{H}) /\{ \pm \mathbf{1}\} \cong \operatorname{Aut}(\mathcal{D})_{0} \text { and } \operatorname{Sp}_{\text {res }}(\mathcal{H}) / \mathrm{U}(\mathcal{H}) \cong \mathcal{D}_{s}
$$

(i) For the complex symmetric bilinear form

$$
\beta_{\mathbb{C}}\left((x, y),\left(x^{\prime}, y^{\prime}\right)\right):=\left\langle x, \sigma y^{\prime}\right\rangle+\left\langle x^{\prime}, \sigma y\right\rangle
$$

we similarly obtain

$$
\mathrm{O}(\mathcal{H}) \cong \mathrm{U}(\mathcal{H}, \mathcal{H}) \cap \mathrm{O}\left(\mathcal{K}, \beta_{\mathbb{C}}\right)
$$

which in turn leads to

$$
\mathrm{O}_{\text {res }}(\mathcal{H}) \cong \mathrm{U}_{\text {res }}(\mathcal{H}, \mathcal{H}) \cap \mathrm{O}\left(\mathcal{K}, \beta_{\mathbb{C}}\right)
$$

and

$$
\mathfrak{o}_{\text {res }}(\mathcal{H}) \cong\left\{\left(\begin{array}{cc}
a & b \\
-b^{*} & -a^{\top}
\end{array}\right): a \in \mathfrak{u}(\mathcal{H}), b^{\top}=-b \in B_{2}(\mathcal{H})\right\} .
$$

Since we shall need it several times in the following, we recall some basic facts on the adjoint and the coadjoint action on a centrally extended Lie algebra.

Remark 3.3. Let $\widehat{\mathfrak{g}}=\mathbb{R} \oplus_{\omega} \mathfrak{g}$ be a central extension of the Lie algebra $\mathfrak{g}$ defined by the 2-cocycle $\omega$, i.e.

$$
\left[(z, x),\left(z^{\prime}, x^{\prime}\right)\right]=\left(\omega\left(x, x^{\prime}\right),\left[x, x^{\prime}\right]\right) \quad \text { for } z, z^{\prime} \in \mathbb{R}, x, x^{\prime} \in \mathfrak{g} .
$$

Then the adjoint action of $\widehat{\mathfrak{g}}$ factors through a representation $\operatorname{ad}_{\widehat{\mathfrak{g}}}: \mathfrak{g} \rightarrow \operatorname{der}(\widehat{\mathfrak{g}})$, given by $\operatorname{ad}_{\widehat{\mathfrak{g}}}(x)(z, y)=(\omega(x, y),[x, y])$, which implies that $\mathfrak{g} \rightarrow \mathfrak{g}^{\prime}, x \mapsto i_{x} \omega=\omega(x, \cdot)$ is a 1-cocycle.

If $G$ is a corresponding Lie group to which the action of $\mathfrak{g}$ on $\widehat{\mathfrak{g}}$ integrates as a smooth linear action, then it is of the form

$$
\operatorname{Ad}_{\widehat{\mathfrak{g}}}(g)(z, y)=\left(z+\Theta_{\omega}(g)(\operatorname{Ad}(g) y), \operatorname{Ad}(g) y\right)=\left(z-\Theta_{\omega}\left(g^{-1}\right)(y), \operatorname{Ad}(g) y\right),
$$

where $\Theta_{\omega}: G \rightarrow \mathfrak{g}^{\prime}$ is a 1-cocycle with $T_{1}\left(\Theta_{\omega}\right) x=i_{x} \omega$. The uniqueness of the representation of $G$ on $\widehat{\mathfrak{g}}$ follows from the general fact that, for a connected Lie 
group, smooth representations are uniquely determined by their derived representations ([66, Remark II.3.7]). The existence for simply connected $G$ follows from [64, Proposition VII.6]. The corresponding dual representation of $G$ on $\widehat{\mathfrak{g}}^{\prime} \cong \mathbb{R} \times \mathfrak{g}^{\prime}$ is then given by

$$
\operatorname{Ad}_{\mathfrak{\mathfrak { g }}}^{*}(g)(z, \alpha)=\left(z, \operatorname{Ad}^{*}(g) \alpha-z \Theta_{\omega}(g)\right) .
$$

As these formulas show, the passage from the adjoint and coadjoint action of $\mathfrak{g}$ to the $G$-action on $\widehat{\mathfrak{g}}$ is completely encoded in the cocycle $\Theta_{\omega}: G \rightarrow \mathfrak{g}^{\prime}$.

\section{Momentum Sets of Smooth Unitary Representations}

In this section we introduce the concept of a semibounded unitary representation of a Lie group $G$. A key tool to study these representations is the momentum map $\Phi: \mathbb{P}\left(\mathcal{H}^{\infty}\right) \rightarrow \mathfrak{g}^{\prime}$. According to Theorem 4.5, this map is also a momentum map in the classical sense of differential geometry, provided $G$ is a Banach-Lie group.

Definition 4.1. A unitary representation of $G$ is a pair $(\pi, \mathcal{H})$ of a complex Hilbert space $\mathcal{H}$ and a group homomorphism $\pi: G \rightarrow \mathrm{U}(\mathcal{H})$. It is said to be continuous if the action map $G \times \mathcal{H} \rightarrow \mathcal{H},(g, v) \mapsto \pi(g) v$ is continuous, which, since $G$ acts by isometries, is equivalent to the continuity of the orbit maps $\pi^{v}: G \rightarrow \mathcal{H}, g \mapsto \pi(g) v$. We write

$$
\mathcal{H}^{\infty}:=\left\{v \in \mathcal{H}: \pi^{v} \in C^{\infty}(G, \mathcal{H})\right\}
$$

for the subspace of smooth vectors. The representation $(\pi, \mathcal{H})$ is said to be smooth if $\mathcal{H}^{\infty}$ is dense in $\mathcal{H}$. On $\mathcal{H}^{\infty}$ the derived representation $\mathrm{d} \pi$ of the Lie algebra $\mathfrak{g}=\mathbf{L}(G)$ is defined by

$$
\mathrm{d} \pi(x) v:=\left.\frac{d}{d t}\right|_{t=0} \pi\left(\exp _{G} t x\right) v
$$

(cf. [62, Remark IV.2]). If $(\pi, \mathcal{H})$ is smooth, then the invariance of $\mathcal{H}^{\infty}$ under $\pi(G)$ implies that the operators $i \mathrm{~d} \pi(x), x \in \mathfrak{g}$, on this space are essentially self-adjoint (cf. [67, Lemma 5.6], [83, Theorem VIII.10]).

Remark 4.2. (a) If $(\pi, \mathcal{H})$ is a smooth unitary representation, then the space $\mathcal{H}^{c}:=\left\{v \in \mathcal{H}: \pi^{v} \in C(G, \mathcal{H})\right\} \supseteq \mathcal{H}^{\infty}$ of continuous vectors is dense, and since this space is closed (by the uniform boundedness of $\pi(G)$ ), it follows that $\mathcal{H}=\mathcal{H}^{c}$. This in turn implies that the $G$-action on $\mathcal{H}$ is continuous. This means that smooth representations are in particular continuous.

(b) If $G$ is finite dimensional, then Gårding's Theorem asserts that every continuous unitary representation of $G$ is smooth. However, this is false for infinite dimensional Lie groups. The representation of the additive Banach-Lie group $G:=L^{2}([0,1], \mathbb{R})$ on $\mathcal{H}=L^{2}([0,1], \mathbb{C})$ by $\pi(g) f:=e^{i g} f$ is continuous with $\mathcal{H}^{\infty}=\{0\}([7])$. 
Definition 4.3. (a) Let $\mathbb{P}\left(\mathcal{H}^{\infty}\right)=\left\{[v]:=\mathbb{C} v: 0 \neq v \in \mathcal{H}^{\infty}\right\}$ denote the projective space of the subspace $\mathcal{H}^{\infty}$ of smooth vectors. The map

$$
\Phi_{\pi}: \mathbb{P}\left(\mathcal{H}^{\infty}\right) \rightarrow \mathfrak{g}^{\prime} \quad \text { with } \Phi_{\pi}([v])(x)=\frac{1}{i} \frac{\langle\mathrm{d} \pi(x) \cdot v, v\rangle}{\langle v, v\rangle}
$$

is called the momentum map of the unitary representation $\pi$. The right-hand side is well defined because it only depends on $[v]=\mathbb{C} v$. The operator $i \mathrm{~d} \pi(x)$ is symmetric so that the right-hand side is real, and since $v$ is a smooth vector, it defines a continuous linear functional on $\mathfrak{g}$. We also observe that we have a natural action of $G$ on $\mathbb{P}\left(\mathcal{H}^{\infty}\right)$ by $g \cdot[v]:=[\pi(g) v]$, and the relation

$$
\pi(g) \mathrm{d} \pi(x) \pi(g)^{-1}=\mathrm{d} \pi(\operatorname{Ad}(g) x)
$$

immediately implies that $\Phi_{\pi}$ is equivariant with respect to the coadjoint action of $G$ on $\mathfrak{g}^{\prime}$.

(b) The weak-*-closed convex hull $I_{\pi} \subseteq \mathfrak{g}^{\prime}$ of the image of $\Phi_{\pi}$ is called the (convex) momentum set of $\pi$. In view of the equivariance of $\Phi_{\pi}$, it is an $\operatorname{Ad}^{*}(G)$ invariant subset of $\mathfrak{g}^{\prime}$.

For the following theorem, we recall the definition of a Hamiltonian action:

Definition 4.4. Let $\sigma: G \times M \rightarrow M$ be a smooth action of the Lie group $G$ on the smooth manifold $M$ (neither is assumed to be finite dimensional) and suppose that $\omega$ is a $G$-invariant closed 2 -form on $M$. Then this action is said to be Hamiltonian if there exists a map $J: \mathfrak{g} \rightarrow C^{\infty}(M, \mathbb{R})$ for which $\mathrm{d} J_{x}=i_{\dot{\sigma}(x)} \omega$ holds for the derived Lie algebra homomorphism $\dot{\sigma}: \mathfrak{g} \rightarrow \mathcal{V}(M)$, defined by $\dot{\sigma}(x)_{p}=-T_{(\mathbf{1}, p)}(\sigma)(x, 0)$. The map $\Phi: M \rightarrow \mathfrak{g}^{\prime}, \Phi(m)(x):=J_{x}(m)$ is then called the corresponding momentum map. Of particular interest are momentum maps which are equivariant with respect to the coadjoint action.

Theorem 4.5. Let $(\pi, \mathcal{H})$ be a unitary representation of a Banach-Lie group $G$. Then the seminorms

$$
p_{n}(v):=\sup \left\{\left\|\mathrm{d} \pi\left(x_{1}\right) \cdots \mathrm{d} \pi\left(x_{n}\right) v\right\|: x_{i} \in \mathfrak{g},\left\|x_{i}\right\| \leq 1\right\}
$$

define on $\mathcal{H}^{\infty}$ the structure of a Fréchet space with respect to which the action of $G$ is smooth. Accordingly, the projective space $\mathbb{P}\left(\mathcal{H}^{\infty}\right)$ carries the structure of a complex Fréchet manifold on which $G$ acts smoothly by holomorphic maps. The Fubini-Study metric on $\mathbb{P}(\mathcal{H})$ induces on $\mathbb{P}\left(\mathcal{H}^{\infty}\right)$ the structure of a weak Kähler manifold whose corresponding weak symplectic form $\Omega$ is given for any unit vector $v \in \mathcal{H}^{\infty}$ by

$$
\Omega_{[v]}\left(T_{v}(q) x, T_{v}(q) y\right)=-2 \operatorname{Im}\langle x, y\rangle \quad \text { for } x, y \in v^{\perp},
$$

where $q: \mathcal{H}^{\infty} \backslash\{0\} \rightarrow \mathbb{P}\left(\mathcal{H}^{\infty}\right), v \mapsto[v]$, is the canonical projection. With respect to this symplectic form, the action of $G$ on $\mathbb{P}\left(\mathcal{H}^{\infty}\right)$ is Hamiltonian with momentum map $\Phi_{\pi}$. 
Proof. The smoothness of the action of $G$ on $\mathcal{H}^{\infty}$ follows from Theorem A.2. This implies that the natural charts defined by projections of affine hyperplanes define on $\mathbb{P}\left(\mathcal{H}^{\infty}\right)$ a complex manifold structure for which $G$ acts smoothly by holomorphic maps (see [62, Proposition V.2] for details). Moreover, the Fubini-Study metric on $\mathbb{P}(\mathcal{H})$ restricts to a (weak) Kähler metric on $\mathbb{P}\left(\mathcal{H}^{\infty}\right)$ which is invariant under the $G$-action. It is determined by the property that the projection map $q$ satisfies for any unit vector $v$ and $x, y \in v^{\perp}$ the relation

$$
\left\langle T_{v}(q) x, T_{v}(q) y\right\rangle=\langle x, y\rangle .
$$

In particular, we see that $\Omega$ defines a weak symplectic 2 -form on $\mathbb{P}\left(\mathcal{H}^{\infty}\right)$ (cf. [53, Sec. 5.3]). For $x \in \mathfrak{g}$, the smooth function

$$
H_{x}([v]):=\frac{1}{i} \frac{\langle\mathrm{d} \pi(x) v, v\rangle}{\langle v, v\rangle}
$$

on $\mathbb{P}\left(\mathcal{H}^{\infty}\right)$ now satisfies for $y \in v^{\perp}$ and $\|v\|=1$ :

$$
\begin{aligned}
\mathrm{d} H_{x}([v])\left(T_{v}(q) y\right) & =2 \operatorname{Re}\langle-i \mathrm{~d} \pi(x) v, y\rangle=2 \operatorname{Im}\langle\mathrm{d} \pi(x) v, y\rangle \\
& =-\Omega_{[v]}\left(T_{v}(q) \mathrm{d} \pi(x) v, T_{v}(q) y\right),
\end{aligned}
$$

i.e. $\mathrm{d} H_{x}=i_{X} \Omega$ for the smooth vector field on $\mathbb{P}\left(\mathcal{H}^{\infty}\right)$ defined by $X([v]):=$ $-T_{v}(q) \mathrm{d} \pi(x) v=\dot{\sigma}(x)([v])$. This means that the action of $G$ on $\mathbb{P}\left(\mathcal{H}^{\infty}\right)$ is Hamiltonian and $\Phi_{\pi}: \mathbb{P}\left(\mathcal{H}^{\infty}\right) \rightarrow \mathfrak{g}^{\prime}$ is a corresponding momentum map.

The main new point of the preceding theorem is that it provides for any BanachLie group a natural analytic setup for which the momentum map $\Phi_{\pi}$ really is a momentum map for a smooth action on a weak symplectic manifold. The corresponding result for the action of the Banach-Lie group $\mathrm{U}(\mathcal{H})$ on $\mathbb{P}(\mathcal{H})$ is well-known (cf. [53, Ex. 11.4(h)]).

A key property of the momentum set $I_{\pi}$ is that it provides complete information on the extreme spectral values of the operators $i \mathrm{~d} \pi(x)$ :

$$
\sup (\operatorname{Spec}(i \mathrm{~d} \pi(x)))=s_{I_{\pi}}(x)=-\inf \left\langle I_{\pi}, x\right\rangle \quad \text { for } x \in \mathfrak{g}
$$

(cf. [67, Lemma 5.6]). This relation immediately entails the equivalences

$$
x \in I_{\pi}^{\star} \Leftrightarrow s_{I_{\pi}}(x) \leq 0 \Leftrightarrow-i \mathrm{~d} \pi(x) \geq 0 .
$$

It is now natural to study those representations for which $s_{\pi}$, resp., the set $I_{\pi}$, contains the most significant information, which leads to the following concept:

Definition 4.6. A smooth unitary representation $(\pi, \mathcal{H})$ of a Lie group $G$ is said to be semibounded if its momentum set $I_{\pi}$ is semi-equicontinuous, i.e. if

$$
s_{\pi}(x):=s_{I_{\pi}}(x)=\sup (\operatorname{Spec}(i \mathrm{~d} \pi(x)))
$$

is bounded from above on some non-empty open subset of $\mathfrak{g}$. We call $(\pi, \mathcal{H})$ bounded if $I_{\pi}$ is equicontinuous. 
The representation $(\pi, \mathcal{H})$ is said to satisfy the positive energy condition with respect to some $d \in \mathfrak{g}$ if $i \mathrm{~d} \pi(d) \geq 0$. It satisfies the positive energy condition with respect to some convex cone $C \subseteq \mathfrak{g}$, if $i \mathrm{~d} \pi(x) \geq 0$ holds for each $x \in C$ (cf. [95]).

For a semibounded representation $(\pi, \mathcal{H})$, the domain $s_{\pi}^{-1}(\mathbb{R})$ of $s_{\pi}$ is a convex cone with non-empty interior and $s_{\pi}$ is continuous on this open cone (cf. [67, Proposition 6.8]). Since the momentum set $I_{\pi}$ is invariant under the coadjoint action, the function $s_{\pi}$ and its domain are invariant under the adjoint action. This leads to two open invariant convex cones in $\mathfrak{g}$ :

$$
W_{\pi}:=\left\{x \in \mathfrak{g}: s_{\pi}(x)<\infty\right\}^{0}=B\left(I_{\pi}\right)^{0} \quad \text { and } \quad C_{\pi}:=\left\{x \in \mathfrak{g}: s_{\pi}(x) \leq 0\right\}^{0},
$$

where " 0 " denotes the interior of a set. Note that (8) implies that $C_{\pi}$ is the interior of the dual cone $I_{\pi}^{\star}$.

We collect some basic properties of these two cones:

Proposition 4.7. For a smooth representation $(\pi, \mathcal{H})$ of $G$, the following assertions hold:

(i) If $C_{\pi} \neq \emptyset$, then $\pi$ is semibounded, and if $i \mathbf{1} \in \mathrm{d} \pi(\mathfrak{g})$, then the converse is also true.

(ii) If $C_{\pi} \neq \emptyset$, then $H\left(C_{\pi}\right)=\operatorname{ker} \mathrm{d} \pi$. In particular, $C_{\pi}$ is pointed if and only if the derived representation $\mathrm{d} \pi$ is injective.

(iii) If $\mathfrak{h} \subseteq \mathfrak{g}$ is a subalgebra with the property that $\left.\mathrm{d} \pi\right|_{\mathfrak{h}}$ is a bounded representation, then $\mathfrak{h} \subseteq H\left(W_{\pi}\right)$.

(iv) Let $\eta: H \rightarrow G$ be a morphism of Lie subgroups. If $\pi$ is semibounded, then $\pi_{H}:=\pi \circ \eta$ is semibounded if $W_{\pi} \cap \mathbf{L}(\eta) \mathfrak{h} \neq \emptyset$, and then $I_{\pi_{H}}=\mathbf{L}(\eta)^{*} I_{\pi}$. In particular, if $H \subseteq G$ is a Lie subgroup, then the restriction $\pi_{H}:=\left.\pi\right|_{H}$ of $\pi$ to $H$ is semibounded if $W_{\pi} \cap \mathfrak{h} \neq \emptyset$, and then $I_{\pi_{H}}=\left.I_{\pi}\right|_{\mathfrak{h}}$.

Proof. (i) If $C_{\pi} \neq \emptyset$, then the boundedness of $s_{\pi}$ on $C_{\pi}$ implies that $\pi$ is semibounded. Suppose, conversely, that $\pi$ is semibounded and $i \mathbf{1} \in \mathrm{d} \pi(\mathfrak{g})$. Then there exists an element $x \in \mathfrak{g}$ for which $s_{\pi}$ is bounded from above on a neighborhood of $x$ by some $M \in \mathbb{R}$. Pick $z \in \mathfrak{g}$ with $\mathrm{d} \pi(z)=i \mathbf{1}$. Now $s_{\pi}(x+M z) \leq 0$ on this neighborhood, so that $C_{\pi} \neq \emptyset$.

(ii) The relation $C_{\pi}=\left(I_{\pi}^{\star}\right)^{0}$ implies that

$$
I_{\pi}^{\star}=\overline{C_{\pi}}
$$

(Lemma 2.8). If $C_{\pi}$ is non-empty, then Lemma 2.9(i) implies that

$$
H\left(C_{\pi}\right)=H\left(I_{\pi}^{\star}\right)=I_{\pi}^{\star} \cap-I_{\pi}^{\star}=I_{\pi}^{\perp}=\operatorname{ker} \mathrm{d} \pi
$$

because $\mathrm{d} \pi(x)=0$ is equivalent to $\operatorname{Spec}(i \mathrm{~d} \pi(x)) \subseteq\{0\}$.

(iii) That $\left.\mathrm{d} \pi\right|_{\mathfrak{h}}$ is bounded implies that all operators $i \mathrm{~d} \pi(x), x \in \mathfrak{h}$, are bounded, so that $\mathfrak{h} \subseteq B\left(I_{\pi}\right) \subseteq \bar{W}_{\pi}=\lim \left(W_{\pi}\right)($ Lemma $2.9(\mathrm{i}))$, and since $\mathfrak{h}=-\mathfrak{h}$, the assertion follows. 
(iv) From (7) it follows that $s_{\pi_{H}}=s_{\pi} \circ \mathbf{L}(\gamma)$, and if $W_{\pi} \cap \mathbf{L}(\gamma) \mathfrak{h} \neq \emptyset$, this function is bounded on some non-empty open subset of $\mathfrak{h}$. Therefore $\pi_{H}$ is semibounded.

Pick $x \in \mathfrak{h}$ with $y:=\mathbf{L}(\gamma) x \in W_{\pi}$. Then the evaluation map $\eta_{y}^{\mathfrak{g}}: I_{\pi} \rightarrow \mathbb{R}, \alpha \mapsto$ $\alpha(x)$, is proper by Proposition 2.7, and this map factors through the adjoint map $\mathbf{L}(\eta)^{\prime}: \mathfrak{g}^{\prime} \rightarrow \mathfrak{h}^{\prime}, \alpha \mapsto \alpha \circ \mathbf{L}(\eta):$

$$
\eta_{y}^{\mathfrak{g}}=\eta_{x}^{\mathfrak{h}} \circ \mathbf{L}(\eta)^{\prime}: I_{\pi} \rightarrow \mathbb{R} .
$$

Since the weak-*-topology on $\mathfrak{h}^{\prime}$ is Hausdorff, [11, Chap. I, Sec. 10, Proposition 1.5] implies that $\mathbf{L}(\eta)^{\prime}: I_{\pi} \rightarrow \mathfrak{h}^{\prime}$ is a proper map. In particular, its image is closed, hence a weak-*-closed convex subset of $\mathfrak{h}^{\prime}$. For each $x \in \mathfrak{h}$ we have

$$
\sup \left\langle I_{\pi_{H}}, x\right\rangle=s_{\pi_{H}}(-x)=s_{\pi}(-\mathbf{L}(\eta) x)=\sup \left\langle I_{\pi}, \mathbf{L}(\eta) x\right\rangle=\sup \left\langle\mathbf{L}(\eta)^{\prime} I_{\pi}, x\right\rangle,
$$

so that the Hahn-Banach Separation Theorem implies that $I_{\pi_{H}}=\mathbf{L}(\eta)^{\prime} I_{\pi}$.

Remark 4.8. If $i \mathbf{1} \notin \mathrm{d} \pi(\mathfrak{g})$, then we may consider the direct product Lie group $\widehat{G}:=G \times \mathbb{T}$, where we consider $\mathbb{T}$ as a subgroup of $\mathbb{C}^{\times}$, with the exponential function $\exp _{\mathbb{T}}(t)=e^{i t}$ on $\mathbf{L}(\mathbb{T}) \cong \mathbb{R}$. Our representation $\pi$ now extends trivially to a smooth unitary representation of $\widehat{G}$, defined by

$$
\widehat{\pi}(g, t):=t \pi(g) .
$$

Now $\widehat{\mathfrak{g}} \cong \mathfrak{g} \times \mathbb{R}, \widehat{\mathfrak{g}}^{\prime} \cong \mathfrak{g}^{\prime} \times \mathbb{R}$, and the momentum map of $\widehat{\pi}$ is given by

$$
\Phi_{\widehat{\pi}}([v])=\left(\Phi_{\pi}([v]), 1\right),
$$

so that $I_{\widehat{\pi}}=I_{\pi} \times\{1\}$. With Remark 2.6, we now see that $\pi$ is semibounded if and only if $C_{\widehat{\pi}} \neq \emptyset$. Note also that $\mathrm{d} \widehat{\pi}(0,1)=i \mathbf{1}$, so that Proposition $4.7(\mathrm{i})$ applies to this representation.

Remark 4.9. (on bounded representations) (a) From [68, Theorem 3.1] we know that a smooth representation $(\pi, \mathcal{H})$ is bounded if and only if $\pi: G \rightarrow \mathrm{U}(\mathcal{H})$ is a morphism of Lie groups, if $\mathrm{U}(\mathcal{H})$ is endowed with the Banach-Lie group structure defined by the operator norm. In view of $(7)$, the boundedness of $(\pi, \mathcal{H})$ is equivalent to the boundedness of all operators $i \mathrm{~d} \pi(x)$ and the continuity of the derived representation $\mathrm{d} \pi: \mathfrak{g} \rightarrow B(\mathcal{H})$ as a morphism of topological Lie algebras, where we identify the operator $\mathrm{d} \pi(x)$ with its continuous extension to all of $\mathcal{H}$.

(b) In [68, Proposition 3.5] it is also shown that if $\pi$ is continuous with respect to the norm topology on $\mathrm{U}(\mathcal{H})$, then $\pi$ is automatically smooth if either $G$ is locally exponential or $\mathfrak{g}$ is a barreled space. In particular, for Banach-Lie groups $G$ the bounded representations are precisely the norm-continuous ones. The concept of a norm-continuous unitary representation also makes sense for arbitrary topological groups, but the refined concept of semiboundedness does not; it requires the Lie algebra for its definition.

(c) If $(\pi, \mathcal{H})$ is a bounded unitary representation of a Lie group $G$, then the closure $\mathfrak{h}$ of $\mathrm{d} \pi(\mathfrak{g}) \subseteq \mathfrak{u}(\mathcal{H})$ is a Banach-Lie algebra, and $H:=\left\langle\exp _{\mathrm{U}(\mathcal{H})} \mathfrak{h}\right\rangle \subseteq \mathrm{U}(\mathcal{H})$ carries a natural Banach-Lie group structure with $\mathbf{L}(H)=\mathfrak{h}([66$, Theorem IV.4.9]). 
If $G$ is connected, then $\pi: G \rightarrow \mathrm{U}(\mathcal{H})$ factors through the inclusion map $H \rightarrow \mathrm{U}(\mathcal{H})$ of this Banach-Lie group. In this sense bounded representations are a "Banach phenomenon" and all questions on this class of representations can be reduced to Banach-Lie groups.

(d) If $(\pi, \mathcal{H})$ is a bounded representation for which $\operatorname{ker}(\mathrm{d} \pi)=I_{\pi}^{\perp}=\{0\}$, then $\|\mathrm{d} \pi(x)\|$ defines a $G$-invariant norm on $\mathfrak{g}$. If $G$ is finite-dimensional, then the existence of an invariant norm implies that the Lie algebra $\mathfrak{g}$ is compact. In particular, all its irreducible representations are finite dimensional. However, in the infinite dimensional context, there is a substantially richer supply of bounded unitary representations. For a detailed discussion of bounded unitary representations of the unitary groups $\mathrm{U}_{p}(\mathcal{H})$ (Examples $3.2(\mathrm{~b})$ ), we refer to [59].

Example 4.10. Let $\mathcal{H}$ be a complex Hilbert space and consider $\mathrm{U}(\mathcal{H})$ as a BanachLie group with Lie algebra $\mathfrak{u}(\mathcal{H})$. Writing $A \ll B$ for $A, B \in B(\mathcal{H})$ if $B-A$ is a positive invertible operator, we see that for the identical representation $(\pi, \mathcal{H})$, the cone

$$
C_{\pi}=\{x \in \mathfrak{u}(\mathcal{H}): i x \ll 0\}
$$

is non-empty. Since $\pi$ is bounded, we also have $W_{\pi}=\mathfrak{g}$. The same holds for all representations $\left(\pi^{\otimes n}, \mathcal{H}^{\otimes n}\right)$ on the $n$-fold tensor power of $\mathcal{H}$.

The natural representations $\pi_{s}$ and $\pi_{a}$ of $\mathrm{U}(\mathcal{H})$ on the symmetric and antisymmetric Fock spaces

$$
S(\mathcal{H}):=\widehat{\oplus}_{n \in \mathbb{N}_{0}} S^{n}(\mathcal{H}) \quad \text { and } \quad \Lambda(\mathcal{H}):=\widehat{\oplus}_{n \in \mathbb{N}_{0}} \Lambda^{n}(\mathcal{H})
$$

are direct sums of bounded representations, hence in particular smooth. They are not bounded, as the restriction to the subgroup $\mathbb{T} \mathbf{1}$ already shows. However, the relation $C_{\pi} \subseteq C_{\pi_{s}}, C_{\pi_{a}}$ still implies that $\pi_{s}$ and $\pi_{a}$ are semibounded. We shall use this simple observation later in our proof that the metaplectic and the spin representation are semibounded (cf. Secs. 9 and 10).

We conclude this section with a convenient tool to verify the existence of eigenvectors for semibounded unitary one-parameter groups.

Proposition 4.11. Let $(\pi, \mathcal{H})$ be a semibounded unitary representation of $G$ and $d \in W_{\pi}$ with $\exp _{G}(\lambda d) \in Z(G)$ for some $\lambda \in \mathbb{R}^{\times}$. Suppose that $\pi\left(\exp _{G}(\lambda d)\right) \in \mathbb{T} \mathbf{1}$, which is in particular the case if $\pi$ is irreducible. Then $i \mathrm{~d} \pi(d)$ is bounded from above, has discrete spectrum, and there exists a smooth vector $v \in \mathcal{H}^{\infty}$ which is an eigenvector for the largest eigenvalue of $i \mathrm{~d} \pi(d)$.

Proof. Replacing $d$ by $\lambda d$, we may assume that $\lambda=1$. If $\pi$ is irreducible, Schur's Lemma implies that $\pi(Z(G)) \subseteq \mathbb{T} \mathbf{1}$ and thus in particular $\pi\left(\exp _{G} d\right) \in \mathbb{T} \mathbf{1}$. Let $\beta \in \mathbb{R}$ with $\pi(\exp d)=e^{i \beta} \mathbf{1}$. Then $e^{\mathrm{d} \pi(d)-i \beta \mathbf{1}}=\mathbf{1}$, so that $\operatorname{Spec}(i \mathrm{~d} \pi(d)+\beta \mathbf{1}) \subseteq 2 \pi \mathbb{Z}$ is discrete. 
Let $P_{n}$ denote the orthogonal projection onto the eigenspace of $i \mathrm{~d} \pi(d)$ corresponding to the eigenvalue $2 \pi n-\beta$. This projection is given by the following integral

$$
P_{n}(v)=\int_{0}^{1} e^{2 \pi i n t} e^{-i t \beta} \pi(\exp (t d)) v d t=\int_{0}^{1} e^{i t(2 \pi n-\beta)} \pi(\exp (t d)) v d t
$$

If $v$ is a smooth vector for $G$, then the smoothness of the $\mathcal{H}$-valued function

$$
g \mapsto \pi(g) P_{n}(v)=\int_{0}^{1} e^{i t(2 \pi n-\beta)} \pi(g \exp (t d)) v d t
$$

follows from the smoothness of the integrand as a function on $\mathbb{R} \times G$ by differentiation under the integral (cf. [25] for details). This implies that $P_{n}\left(\mathcal{H}^{\infty}\right) \subseteq \mathcal{H}^{\infty}$.

Therefore the density of $\mathcal{H}^{\infty}$ in $\mathcal{H}$ implies the density of $P_{n}\left(\mathcal{H}^{\infty}\right)$ in $P_{n}(\mathcal{H})$. In particular, each non-zero eigenspace of $\mathrm{d} \pi(d)$ contains smooth vectors. Finally, the boundedness of $\operatorname{Spec}(i \mathrm{~d} \pi(d))$ from above implies the existence of a maximal eigenvalue.

\section{Aspects of Complex Analysis}

As we have already seen in [68], a closer analysis of semibounded representations requires a good understanding of the related complex geometric structures. Basically, the connection to complex analysis relies on the fact that if $A$ is a self-adjoint operator on a complex Hilbert space, then the associated unitary oneparameter group $\gamma_{A}(t):=e^{i t A}$ extends to a strongly continuous homomorphism $\hat{\gamma}: \mathbb{C}_{+}:=\mathbb{R}+i \mathbb{R}_{+} \rightarrow B(\mathcal{H}), z \mapsto e^{i z A}$ (defined by a spectral integral) which is holomorphic on the open upper half plane if and only if $\operatorname{Spec}(A)$ is bounded from below. It is this key observation, applied in various situations, that leads to a variety of interesting tools and results, that we describe below.

Problem 5.1. If $(\pi, \mathcal{H})$ is a semibounded unitary representation, then we have a well-defined map

$$
\widehat{\pi}: G \times W_{\pi} \rightarrow B(\mathcal{H}), \quad(g, x) \mapsto g e^{i \mathrm{~d} \pi(x)},
$$

and if $G$ is finite dimensional and $\mathrm{d} \pi$ injective, then we know from [60] that the product set $S_{\pi}:=G \times W_{\pi}$ always carries a complex manifold structure, a holomorphic associative multiplication and an antiholomorphic involution $(g, x)^{*}:=$ $\left(g^{-1}, \operatorname{Ad}(g) x\right)$, turning $\left(S_{\pi}, *\right)$ into a complex involutive semigroup and $\widehat{\pi}$ into a holomorphic representation. In particular, $\widehat{\pi}\left(G \times W_{\pi}\right)=\pi(G) e^{i \mathrm{~d} \pi\left(W_{\pi}\right)}$ is an involutive subsemigroup of $B(\mathcal{H})$. This structure is extremely useful in the theory of semibounded representations and the related geometry. However, for infinite dimensional groups, our understanding of corresponding analogs is still quite rudimentary.

As we shall see below, the preceding problem has a trivial solution for Abelian groups. 


\subsection{The Abelian case}

If $E$ is a locally convex space, then its additive group $G:=(E,+)$ is a particularly simple locally convex Lie group. Then $\mathbf{L}(G)=E$ as an Abelian Lie algebra, $\exp _{G}=$ $\mathrm{id}_{E}$, and the coadjoint action is trivial.

For such groups semibounded representations can be understood completely with classical methods, well known from the context of locally compact Abelian groups. If $X \subseteq E^{\prime}$ is a weak-*-closed convex semi-equicontinuous subset, then Proposition 2.7 implies that it is locally compact, so that the space $C_{0}(X)$ of continuous complex-valued functions on $X$ vanishing at infinity is a commutative $C^{*}$-algebra. Moreover, the interior $B(X)^{0}$ is a non-empty open convex cone in $X$, so that $S:=E+i B(X)^{0}$ is a tube domain in the complexification $E_{\mathbb{C}}$. This open subset of $E_{\mathbb{C}}$ is a complex manifold, a semigroup with respect to addition, and $(x+i y)^{*}:=-x+i y$ defines an antiholomorphic involution, turning it into a complex involutive semigroup (cf. [67] for an extended discussion of this technique). This leads to a holomorphic homomorphism of semigroups

$$
\gamma: S \rightarrow C_{0}(X), \quad \gamma(x+i y)(\alpha):=e^{i \alpha(x)-\alpha(y)}
$$

(cf. Proposition 2.7), and one can show that every semibounded smooth representation $(\pi, \mathcal{H})$ of $G$ with $I_{\pi} \subseteq X$ "extends" holomorphically to $S$ by $\widehat{\pi}(x+i y):=$ $\pi(x) e^{i \mathrm{~d} \pi(y)}$, which in turn extends to a representation $\rho$ of the $C^{*}$-algebra $C_{0}(X)$ on $\mathcal{H}$. Using this correspondence and the Spectral Theorem for $C^{*}$-algebras, one arrives at the following spectral theorem ([68, Theorem 4.1]):

Theorem 5.2. (Spectral Theorem for semibounded Representations) For every regular Borel spectral measure $P$ on $X$, the prescription $\pi(v):=P\left(e^{i \eta_{v}}\right)$ (where the right-hand side denotes a spectral integral) defines a semibounded smooth representation of $G$ with $I_{\pi} \subseteq X$. Conversely, any such semibounded representation has this form for a uniquely determined regular Borel spectral measure $P$ on $X$.

The preceding theorem provides a complete description of the semibounded representation theory of Abelian Lie groups of the form $(E,+)$ and hence also of quotients thereof. In particular, every connected Abelian Banach-Lie group is such a quotient. For recent results concerning extremely general spectral theorems for representations of commutative involutive algebras by unbounded operators we refer to [99].

Remark 5.3. With Theorem 5.2 it is easy to see that the bounded unitary representations of $(E,+)$ are precisely those defined by spectral measures on (compact) equicontinuous subsets of the dual space.

Similar characterizations are known for continuous isometric actions $\alpha: G \rightarrow$ $\operatorname{Iso}(E)$ of a locally compact Abelian group $G$ on a Banach space $E$. One associates to such a representation its Arveson spectrum $\operatorname{Sp}(\alpha) \subseteq \widehat{G}$ and then the norm continuity of $\alpha$ is equivalent to the compactness of its spectrum ([79, Theorem 8.1.12]). 
Remark 5.4. The key point behind the Spectral Theorem 5.2 is that the semiboundedness provides a method to connect representations of $(E,+)$ to representations of a commutative $C^{*}$-algebra, so that the Spectral Theorem for commutative Banach-*-algebras provides the spectral measure on $X \subseteq E^{\prime}$.

In general, continuous unitary representations of locally convex spaces cannot be represented in terms of spectral measures on $E^{\prime}$. This is closely related to the problem of writing the continuous positive definite functions $\pi^{v, v}(x):=\langle\pi(x) v, v\rangle$ as the Fourier transform

$$
\hat{\mu}(x)=\int_{E^{\prime}} e^{i \alpha(x)} d \mu(\alpha)
$$

of some finite measure $\mu$ on $E^{\prime}$. If $E$ is nuclear, then the Bochner-Minlos Theorem ([22]) ensures the existence of such measures and hence also of spectral measures representing unitary representations. However, if $E$ is an infinite dimensional Banach space, then $E$ is not nuclear ([22]), so that the Bochner-Minlos Theorem does not apply. Therefore it is quite remarkable that nuclearity assumptions are not needed to deal with semibounded representations.

\subsection{Hilbert spaces of holomorphic functions}

In general, the momentum set of a semibounded representation is not easy to compute, but in many interesting situations it is the weak-*-closed convex hull of a single coadjoint orbit $\mathcal{O}_{\lambda} \subseteq \mathfrak{g}^{\prime}$. Here our intuition is guided by the finite dimensional case, which is by now well understood (cf. [60]).

Remark 5.5. For a finite dimensional connected Lie group $G$, semibounded unitary representations are direct integrals of irreducible ones ([60, Sec. XI.6]) and the irreducible ones possess various kinds of nice structures. Here the key result is that for every irreducible semibounded representation $(\pi, \mathcal{H})$ and $x \in B\left(I_{\pi}\right)^{0}$, we can minimize the proper functional $\eta_{x}(\alpha)=\alpha(x)$ on the convex set $I_{\pi}$ (Proposition 2.7). One can even show that the minimal value is taken in $\Phi_{\pi}([v])$ for an analytic vector $v$, and from that one can derive that $v$ is an eigenvector for $i \mathrm{~d} \pi(x)$ (this is implicitly shown in [60, Theorem X.3.8]). Combining this with finite dimensional structure theory, based on the observation that the adjoint image of the centralizer $Z_{G}(x)$ has compact closure, one can show that if $\mathrm{d} \pi$ is injective, then $\mathcal{H}^{\infty}$ contains a dense highest weight module of the complexification $\mathfrak{g}_{\mathbb{C}}([60$, Theorems X.3.9 and XI.4.5]).

If $\left[v_{\lambda}\right] \in \mathbb{P}\left(\mathcal{H}^{\infty}\right)$ is a highest weight vector for the highest weight representation $\left(\pi_{\lambda}, \mathcal{H}_{\lambda}\right)$, then the corresponding $G$-orbit $G\left[v_{\lambda}\right]$ has the remarkable property that it is a complex homogeneous subspace of $\mathbb{P}\left(\mathcal{H}^{\infty}\right)$, and one can even show that it is the unique $G$-orbit in $\mathbb{P}\left(\mathcal{H}^{\infty}\right)$ with this property ([60, Theorem XV.2.11]). Its image $\mathcal{O}_{\lambda}:=\Phi_{\pi_{\lambda}}\left(G\left[v_{\lambda}\right]\right) \subseteq \mathfrak{g}^{*}$ is a coadjoint orbit satisfying

$$
\mathcal{O}_{\lambda}=\operatorname{Ext}\left(I_{\pi_{\lambda}}\right), \quad I_{\pi_{\lambda}}=\operatorname{conv}\left(\mathcal{O}_{\lambda}\right) \quad \text { and } \quad G\left[v_{\lambda}\right]=\Phi_{\pi_{\lambda}}^{-1}\left(\mathcal{O}_{\lambda}\right)
$$

([60, Theorem X.4.1]), where we write $\operatorname{Ext}(C)$ for the set of extreme points of a convex set $C$. Moreover, two irreducible semibounded representations are equivalent 
if and only if the corresponding momentum sets, resp., the coadjoint orbits $\mathcal{O}_{\lambda}$ coincide ([60, Theorem X.4.2]).

In particular, a central feature of unitary highest weight representations is that the image of the highest weight orbit already determines the momentum set as its closed convex hull. It is therefore desirable to understand in which situations certain $G$-orbits in $\mathbb{P}\left(\mathcal{H}^{\infty}\right)$ already determine the momentum set as the closed convex hull of their image. As we shall see below, this situation frequently occurs if $\mathcal{H}$ consists of holomorphic functions on some complex manifold, resp., holomorphic sections of a line bundle.

Definition 5.6. Let $M$ be a complex manifold (modeled on a locally convex space) and $\mathcal{O}(M)$ the space of holomorphic complex-valued functions on $M$. We write $\bar{M}$ for the conjugate complex manifold. A holomorphic function

$$
K: M \times \bar{M} \rightarrow \mathbb{C}
$$

is said to be a reproducing kernel of a Hilbert subspace $\mathcal{H} \subseteq \mathcal{O}(M)$ if for each $w \in M$ the function $K_{w}(z):=K(z, w)$ is contained in $\mathcal{H}$ and satisfies

$$
\left\langle f, K_{z}\right\rangle=f(z) \quad \text { for } z \in M, f \in \mathcal{H} .
$$

Then $\mathcal{H}$ is called a reproducing kernel Hilbert space and since it is determined uniquely by the kernel $K$, it is denoted $\mathcal{H}_{K}$ (cf. [60, Sec. I.1]).

Now let $G$ be a real Lie group and $\sigma: G \times M \rightarrow M,(g, m) \mapsto g \cdot m$ be a smooth right action of $G$ on $M$ by holomorphic maps. Then $(g . f)(m):=f\left(g^{-1} \cdot m\right)$ defines a unitary representation of $G$ on a reproducing kernel Hilbert space $\mathcal{H}_{K} \subseteq \mathcal{O}(M)$ if and only if the kernel $K$ is invariant:

$$
K(g . z, g \cdot w)=K(z, w) \quad \text { for } z, w \in M, g \in G .
$$

In this case we call $\mathcal{H}_{K}$ a $G$-invariant reproducing kernel Hilbert space and write $\left(\pi_{K}(g) f\right)(z):=f\left(g^{-1} . z\right)$ for the corresponding unitary representation of $G$ on $\mathcal{H}_{K}$.

Theorem 5.7. ([68, Theorem 2.7]) Let $G$ be a Fréchet-Lie group acting smoothly by holomorphic maps on the complex manifold $M$ and $\mathcal{H}_{K} \subseteq \mathcal{O}(M)$ be a $G$-invariant reproducing kernel Hilbert space on which the representation is semibounded, so that the cone $W_{\pi_{K}}$ is not empty. If, for each $x \in W_{\pi_{K}}$, the action $(m, t) \mapsto \exp _{G}(-t x) . m$ of $\mathbb{R}$ on $M$ extends to a holomorphic action of the upper half plane $\mathbb{C}_{+}$(continuous on $\mathbb{C}_{+}$and holomorphic on its interior), then

$$
I_{\pi_{K}}=\overline{\operatorname{conv}}\left(\Phi_{\pi_{K}}\left(\left\{\left[K_{m}\right]: K_{m} \neq 0\right\}\right)\right) .
$$

Example 5.8. Here is the prototypical example that shows why $x \in W_{\pi_{K}}$ is closely related to the existence of a holomorphic extension of the corresponding flow on $M$.

Let $(\pi, \mathcal{H})$ be a continuous unitary representation of $G=\mathbb{R}$ and $\mathcal{H}^{\infty}$ be the Fréchet space of smooth vectors on which $G$ acts smoothly (Theorem A.2). Let 
$M:=\overline{\mathcal{H}^{\infty}}$ be the complex Fréchet manifold obtained by endowing $\mathcal{H}^{\infty}$ with the opposite complex structure. Then $G$ acts on $M$ by holomorphic maps and the density of $\mathcal{H}^{\infty}$ in $\mathcal{H}$ yields an embedding

$$
\iota: \mathcal{H} \hookrightarrow \mathcal{O}(M), \quad \iota(v)(m)=\langle v, m\rangle
$$

whose image is the reproducing kernel space $\mathcal{H}_{K}$ with kernel $K(x, y)=\langle y, x\rangle$. With $A:=-i \mathrm{~d} \pi(1)$ we then have $\pi(t)=e^{i t A}$, and if $\operatorname{Spec}(A)$ is bounded from below, then $\widehat{\pi}: \mathbb{C}_{+} \rightarrow B(\mathcal{H}), z \mapsto e^{z A}$ defines a holomorphic extension of the unitary representation to $\mathbb{C}_{+}$(cf. [60, Theorem VI.5.3]). Since $\mathcal{H}^{\infty}$ is invariant under every operator $\widehat{\pi}(z)$, it is easy to see that $(z, m) \mapsto e^{-\bar{z} A} m$ defines a holomorphic action of $\mathbb{C}_{+}$on $M$, extending the action of $\mathbb{R}$ given by $(t, m) \mapsto \pi(-t) m$.

Remark 5.9. Suppose that $q: \mathbb{V} \rightarrow M$ is a $G$-homogeneous holomorphic Hilbert bundle, i.e. $\mathbb{V}$ carries a left action of $G$ by holomorphic bundle automorphisms and $q(g . z)=g . q(z)$ for $z \in \mathbb{V}, g \in G$. We write $\Gamma(\mathbb{V})$ for the space of holomorphic section of $\mathbb{V}$. Then $G$ acts on $\Gamma(\mathbb{V})$ by $(g . s)(m):=g . s\left(g^{-1} . m\right)$. We are interested in $G$-invariant Hilbert subspaces $\mathcal{H} \subseteq \Gamma(\mathbb{V})$ on which $G$ acts unitarily and for which the evaluation maps $\mathrm{ev}_{m}: \mathcal{H} \rightarrow \mathbb{V}_{m}, s \mapsto s(m), m \in M$, are continuous linear maps between Hilbert spaces.

To see how Theorem 5.7 applies in this situation, we realize $\Gamma(\mathbb{V})$ by holomorphic functions on the dual bundle $\mathbb{V}^{*}$ whose fiber $\left(\mathbb{V}^{*}\right)_{m}$ is the dual space of $\mathbb{V}_{m}$. Each $s \in$ $\Gamma(\mathbb{V})$ defines a holomorphic function $\hat{s}\left(\alpha_{m}\right):=\alpha_{m}(s(m))$ on $\mathbb{V}^{*}$ which is fiberwise linear. We thus obtain an embedding $\Psi: \Gamma(\mathbb{V}) \rightarrow \mathcal{O}\left(\mathbb{V}^{*}\right)$ whose image consists of those holomorphic functions on $\mathbb{V}^{*}$ which are fiberwise linear. Accordingly, $\Psi(\mathcal{H}) \subseteq$ $\mathcal{O}\left(\mathbb{V}^{*}\right)$ is a reproducing kernel Hilbert space. The natural action of $G$ on $\mathbb{V}^{*}$ is given by $\left(g . \alpha_{m}\right)\left(z_{g \cdot m}\right):=\alpha_{m}\left(g^{-1} \cdot z_{g . m}\right)$ for $\alpha_{m} \in \mathbb{V}_{m}^{*}$, so that

$$
\Psi(g . s)\left(\alpha_{m}\right)=\alpha_{m}\left(g . s\left(g^{-1} \cdot m\right)\right)=\left(g^{-1} \cdot \alpha_{m}\right)\left(s\left(g^{-1} \cdot m\right)\right)=\Psi(s)\left(g^{-1} \cdot \alpha_{m}\right)
$$

implies that $\Psi$ is equivariant with respect to the natural $G$-actions on $\Gamma(\mathbb{V})$ and $\mathcal{O}\left(\mathbb{V}^{*}\right)$. Therefore the reproducing kernel $K$ of $\mathcal{H}_{K}:=\Psi(\mathcal{H}) \subseteq \mathcal{O}\left(\mathbb{V}^{*}\right)$ is $G$-invariant, and we are thus in the situation of Theorem 5.7. In addition, the fiberwise linearity of the functions in $\mathcal{H}_{K}$ leads to $K_{z \alpha}=\bar{z} K_{\alpha}$ for $\alpha \in \mathbb{V}^{*}$ and $z \in \mathbb{C}^{\times}$. If $\mathcal{H}_{K} \neq\{0\}$, then the homogeneity of the bundle $\mathbb{V}$ implies that $K_{\alpha} \neq 0$ for every $0 \neq \alpha \in$ $\mathbb{V}^{*}$. Writing $\mathbb{P}\left(\mathbb{V}^{*}\right)$ for the projective bundle associated to $\mathbb{V}^{*}$ whose fibers are the projective spaces of the fibers of $\mathbb{V}^{*}$, we therefore obtain a well-defined map

$$
\Phi: \mathbb{P}\left(\mathbb{V}^{*}\right) \rightarrow \mathfrak{g}^{\prime}, \quad\left[\alpha_{m}\right] \mapsto \Phi_{\pi_{K}}\left(\left[K_{\alpha_{m}}\right]\right) \quad \text { for } \alpha_{m} \in \mathbb{V}_{m}^{*} \backslash\{0\}
$$

Since this map is $G$-equivariant and $G$ acts transitively on $M$, we obtain for each $m_{0} \in M$ :

$$
\Phi\left(\mathbb{P}\left(\mathbb{V}^{*}\right)\right)=\operatorname{Ad}^{*}(G) \Phi\left(\mathbb{P}\left(\mathbb{V}_{m_{0}}^{*}\right)\right)
$$


If, in addition, the requirements of Theorem 5.7 are satisfied, i.e. the action of the one-parameter-groups generated by $-x \in B\left(I_{\pi_{K}}\right)^{0}$ on $\mathbb{V}$, resp., $\mathbb{V}^{*}$, extend holomorphically to $\mathbb{C}_{+}$, we obtain

$$
I_{\pi_{K}}=\overline{\operatorname{conv}}(\operatorname{im}(\Phi)) .
$$

If, in particular, $\mathbb{V}$ is a line bundle, i.e. the fibers are one-dimensional, then $\mathbb{P}\left(\mathbb{V}^{*}\right) \cong M$, and we obtain a $G$-equivariant map $\Phi: M \rightarrow \mathfrak{g}^{\prime}$ whose image is a single coadjoint orbit $\mathcal{O}_{\pi}$.

Example 5.10. If $\mathcal{H}$ is a complex Hilbert space, then its projective space $\mathbb{P}(\mathcal{H})$ is a complex Hilbert manifold. Moreover, there exists a holomorphic line bundle $q: \mathbb{L}_{\mathcal{H}} \rightarrow \mathbb{P}(\mathcal{H})$ with the property that for every nonzero continuous linear functional $\alpha \in \mathcal{H}^{\prime}$ we have on the open subset $U_{\alpha}:=\{[v] \in \mathbb{P}(\mathcal{H}): \alpha(v) \neq 0\}$ a bundle chart

$$
\varphi_{\alpha}:\left.\left(\mathbb{L}_{\mathcal{H}}\right)\right|_{U_{\alpha}} \rightarrow U_{\alpha} \times \mathbb{C}
$$

such that the transition functions are given by

$$
\varphi_{\beta} \circ \varphi_{\alpha}^{-1}([v], z)=\left([v], \frac{\alpha(v)}{\beta(v)}\right) \quad \text { for } 0 \neq \alpha, \beta \in \mathcal{H}^{\prime} .
$$

This implies that each $0 \neq v \in \mathcal{H}$ defines a linear functional on the fiber $\left(\mathbb{L}_{\mathcal{H}}\right)_{[v]}$ by

$$
\varphi_{\alpha}^{-1}([v], z) \mapsto \alpha(v) z,
$$

which further implies that $\left(\mathbb{L}_{\mathcal{H}}\right)_{[v]}^{*}=[v]$, i.e. $\mathbb{L}_{\mathcal{H}}^{*}$ is the tautological bundle over $\mathbb{P}(\mathcal{H})$.

The complement of the zero-section of $\mathbb{L}_{\mathcal{H}}$ is equivalent, as a $\mathbb{C}^{\times}$-bundle, to the projection $\mathcal{H} \backslash\{0\} \rightarrow \mathbb{P}(\mathcal{H})$ by the map $\varphi_{\alpha}([v], z) \mapsto \frac{1}{z \alpha(v)} v$. This identification can be used to show that the natural map

$$
\Psi: \mathcal{H}^{\prime} \rightarrow \Gamma\left(\mathbb{L}_{\mathcal{H}}\right), \quad \Psi(\alpha)([v])=\varphi_{\beta}^{-1}\left([v], \frac{\alpha(v)}{\beta(v)}\right) \quad \text { for } \beta(v) \neq 0
$$

defines a linear isomorphism (see [62, Theorem V.4] for details).

As the group $\mathrm{U}(\mathcal{H})$ acts smoothly by holomorphic bundle isomorphisms on $\mathbb{L}_{\mathcal{H}}$, this construction shows that the unitary representation $\pi^{*}: \mathrm{U}(\mathcal{H}) \rightarrow \mathrm{U}\left(\mathcal{H}^{\prime}\right)$, given by $\pi^{*}(g) \alpha=\alpha \circ \pi(g)^{*}$ can be realized in the space $\Gamma\left(\mathbb{L}_{\mathcal{H}}\right)$ of holomorphic sections of $\mathbb{L}_{\mathcal{H}}$.

To realize the identical representation on $\mathcal{H}$ itself by holomorphic sections, we simply exchange the role of $\mathcal{H}$ and $\mathcal{H}^{\prime}$, which leads to a $\mathrm{U}(\mathcal{H})$-equivariant isomorphism $\mathcal{H} \rightarrow \Gamma\left(\mathbb{L}_{\mathcal{H}^{\prime}}\right)$.

To simplify the applications of Theorem 5.7, we need a criterion for its applicability. Here the main idea is that, since every Hilbert space $\mathcal{H}$ can be realized as a space of holomorphic sections of the bundle $\mathbb{L}_{\mathcal{H}^{\prime}}$, we obtain similar realizations from cyclic $G$-orbits in $\mathbb{P}(\mathcal{H})$ which are complex manifolds.

Theorem 5.11. (Complex Realization Theorem) (a) Let $G$ be a Fréchet-Lie group with Lie algebra $\mathfrak{g}$ and $H \subseteq G$ be a closed subgroup for which the coset space $G / H$ 
carries the structure of a complex manifold such that the projection $q: G \rightarrow G / H$ is a smooth $H$-principal bundle and $G$ acts on $G / H$ by holomorphic maps. Let $x_{0}=\mathbf{1} H \in G / H$ be the canonical base point and $\mathfrak{p} \subseteq \mathfrak{g}_{\mathbb{C}}$ be the kernel of the complex linear extension of the map $\mathfrak{g} \rightarrow T_{x_{0}}(G / H)$ to $\mathfrak{g}_{\mathbb{C}}$, so that $\mathfrak{p}$ is a closed subalgebra of $\mathfrak{g}_{\mathbb{C}}$.

Let $(\pi, \mathcal{H})$ be a unitary representation of $G$ and $\mathrm{d} \pi: \mathfrak{g}_{\mathbb{C}} \rightarrow \operatorname{End}\left(\mathcal{H}^{\infty}\right)$ be the complex linear extension of the derived representation. Suppose that $0 \neq v \in \mathcal{H}^{\infty}$ is an eigenvector for $H$ and of the subalgebra $\overline{\mathfrak{p}}:=\{\overline{x+i y}=x-i y: x+i y \in \mathfrak{p}\}$ of $\mathfrak{g}_{\mathbb{C}}$. Then the map

$$
\eta: G / H \rightarrow \mathbb{P}\left(\mathcal{H}^{\prime}\right), \quad g H \mapsto\left[\pi^{*}(g) \alpha_{v}\right]=\left[\alpha_{v} \circ \pi(g)^{-1}\right], \quad \alpha_{v}(w)=\langle w, v\rangle,
$$

is holomorphic and $G$-equivariant. If, in addition, $v$ is cyclic, then we obtain a $G$ equivariant injection $\mathcal{H} \hookrightarrow \Gamma\left(\eta^{*} \mathbb{L}_{\mathcal{H}^{\prime}}\right)$, where $\eta^{*} \mathbb{L}_{\mathcal{H}^{\prime}}$ is a $G$-equivariant holomorphic line bundle over $G / H$. If, in addition, $G$ is connected, then $\pi$ is irreducible.

(c) Suppose that, for each $x \in W_{\pi}$, the flow $(t, m) \mapsto \exp _{G}(-t x) H$ extends holomorphically to $\mathbb{C}_{+}$. Then the momentum set is given by

$$
I_{\pi}=\overline{\operatorname{conv}}\left(\Phi_{\pi}(G[v])\right)=\overline{\operatorname{conv}}\left(\mathcal{O}_{\Phi_{\pi}([v])}\right) .
$$

Proof. (a) First we observe that the map $\Upsilon: \mathcal{H} \rightarrow \mathcal{H}^{\prime}, v \mapsto \alpha_{v}$ is an antilinear isometry and that the contragredient representation $\pi^{*}(g) \alpha:=\alpha \circ \pi(g)^{-1}$ is also unitary.

Next we recall that the smooth action of $G$ on $M:=G / H$ defines a homomorphism $\mathfrak{g} \rightarrow \mathcal{V}_{\mathcal{O}}(M)$, the Lie algebra of holomorphic vector fields. Since $M$ is complex, $\mathcal{V}_{\mathcal{O}}(M)$ is a complex Lie algebra, and, for each $p \in M$, the subspace $\left\{X \in \mathcal{V}_{\mathcal{O}}(M): X(p)=0\right\}$ is a complex subalgebra. This proves that $\mathfrak{p}$ is a Lie subalgebra of $\mathfrak{g}_{\mathbb{C}}$, and its closedness follows from the continuity of the map $\mathfrak{g}_{\mathbb{C}} \rightarrow T_{x_{0}}(M) \cong \mathfrak{g} / \mathfrak{h}$.

(b) Since $v$ is a $\overline{\mathfrak{p}}$-eigenvector, there exists a continuous linear functional $\lambda: \mathfrak{p} \rightarrow \mathbb{C}$ with $\mathrm{d} \pi(\bar{z}) v=\overline{\lambda(z)} v$ for $z \in \mathfrak{p}$, so that we have for $w \in \mathcal{H}$ and $z \in \mathfrak{p}$ the relation

$$
\alpha_{v}(z . w)=\langle\mathrm{d} \pi(z) w, v\rangle=-\langle w, \mathrm{~d} \pi(\bar{z}) v\rangle=-\lambda(z) \alpha_{v}(w) .
$$

We conclude that $\alpha_{v}$ is an $\mathfrak{p}$-eigenvector. This implies that the tangent map

$$
T_{x_{0}}(\eta): T_{x_{0}}(M) \rightarrow T_{\left[\alpha_{v}\right]}\left(\mathbb{P}\left(\mathcal{H}^{\prime}\right)\right)
$$

is complex linear, i.e. compatible with the respective complex structures. Since $M$ is homogeneous, $T(\eta)$ is complex linear on each tangent space, and this means that $\eta$ is holomorphic. Therefore $\eta^{*} \mathbb{L}_{\mathcal{H}^{\prime}}$ is a holomorphic line bundle over $M$ and we obtain a $G$-equivariant pullback map $\mathcal{H} \cong \Gamma\left(\mathbb{L}_{\mathcal{H}^{\prime}}\right) \rightarrow \Gamma\left(\eta^{*} \mathbb{L}_{\mathcal{H}^{\prime}}\right)$. Its kernel consists of all those sections vanishing on $\eta(M)$, which corresponds to the elements $w \in(\pi(G) v)^{\perp}$. In particular, this map is injective if $v$ is a cyclic vector.

That $(\pi, \mathcal{H})$ is irreducible if $G$ is connected follows from [60, Proposition XV.2.7].

(c) If $x \in W_{\pi}$, then $i \mathrm{~d} \pi(x)$ is bounded from above, so that $(z, \alpha) \mapsto \alpha \circ e^{z \mathrm{~d} \pi(x)}$ defines a continuous action of $\mathbb{C}_{+}$on $\mathcal{H}^{\prime}$ which is holomorphic on $\mathbb{C}_{+}^{0}$ and satisfies 
$(t, \alpha) \mapsto \pi^{*}\left(\exp _{G}(-t x)\right) \alpha$ (cf. Example 5.8). As $\eta$ is $G$-equivariant and holomorphic, it is also equivariant with respect to the $\mathbb{C}_{+}$-actions on $M$ and the holomorphic action on $\mathbb{P}\left(\mathcal{H}^{\prime}\right)$ by $(z,[\alpha]) \mapsto\left[\alpha \circ e^{z \mathrm{~d} \pi(x)}\right]$. Therefore the $\mathbb{C}_{+}$actions on $M$ and on $\mathcal{H}^{\prime}$ combine to a holomorphic action of $\mathbb{C}_{+}$on $\eta^{*} \mathbb{L}_{\mathcal{H}^{\prime}}$. Now we combine Theorem 5.7 with Remark 5.9 to obtain (c). Here we only have to observe that the realization of $\mathcal{H}$ by holomorphic sections of $\mathbb{L}_{\mathcal{H}^{\prime}}$ leads on the dual bundle, whose complement of the zero-section can be identified with $\mathcal{H}^{\prime} \backslash\{0\}$, to the evaluation functional $K_{\alpha_{v}}=v \in \mathcal{H}$ (cf. Example 5.10).

Example 5.12. An important special case where the requirements of Theorem 5.11 are satisfied occurs if the $G$-action on the complex manifold $M$ extends to a holomorphic action of a complex Lie group $G_{\mathbb{C}} \supseteq G$ with holomorphic exponential function.

If $(\pi, \mathcal{H})$ is an irreducible representation of a compact Lie group $G$, then it is finite dimensional and in particular bounded, so that $\pi$ extends to a holomorphic representation $\widehat{\pi}: G_{\mathbb{C}} \rightarrow \operatorname{GL}(\mathcal{H})$. Since the highest weight orbit $G\left[v_{\lambda}\right]$ is a

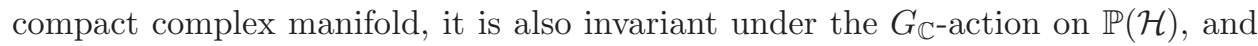
Theorem 5.11, applied to the maximal torus $H \subseteq G$, implies that $I_{\pi}=\operatorname{conv}\left(\mathcal{O}_{\pi}\right)$ for $\mathcal{O}_{\pi}=\Phi_{\pi}\left(G\left[v_{\lambda}\right]\right)$.

Remark 5.13. (a) If $G$ is finite dimensional, then Theorem 5.7 applies to all irreducible semibounded representations ([60, Proposition XII.3.6]), where $G / H$ is the highest weight orbit $G\left[v_{\lambda}\right] \subseteq \mathbb{P}\left(\mathcal{H}_{\lambda}\right)$, and this eventually leads to $I_{\pi_{\lambda}}=$ $\overline{\operatorname{conv}}\left(\mathcal{O}_{-i \lambda}\right)$.

(b) We have already seen in (10) that, for finite dimensional groups, the highest weight orbit $G\left[v_{\lambda}\right] \subseteq \mathbb{P}\left(\mathcal{H}_{\lambda}\right)$ can be characterized as the inverse image of the set $\operatorname{Ext}\left(I_{\pi_{\lambda}}\right)$ of extreme points of the momentum set (Remark 5.5). For the special case where $G$ is a compact group, one finds in [32] a different characterization of the highest weight orbit as the set of all those elements for which the subrepresentation of $\operatorname{Sym}^{2}\left(\mathcal{H}_{\lambda}\right)$ generated by $v \otimes v$ is irreducible. It would be interesting to see if a similar result holds in other situations.

If $G$ is finite dimensional, for any $[v] \in G\left[v_{\lambda}\right]$ the orbit of $[v \otimes v]$ is a complex manifold (actually a holomorphic image of $G\left[v_{\lambda}\right]$ ), and from that one can derive that the cyclic representation it generates is irreducible ([60, Proposition XV.2.7]).

Another interesting characterization of the highest weight orbit of an irreducible representation of a compact Lie group is given in [19]. Starting with an orthonormal basis $i F_{1}, \ldots, i F_{n}$ of the Lie algebra $\mathfrak{g}$ with respect to an invariant scalar product, one defines the invariant dispersion of a state $[v]$ by

$$
(\Delta F)^{2}=\left\langle\sum_{r}\left(F_{r}-\left\langle F_{r}\right\rangle\right)^{2}\right\rangle, \quad \text { where }\langle A\rangle=\frac{\langle A v, v\rangle}{\langle v, v\rangle} \quad \text { for } A=A^{*}
$$


If $C:=\sum_{r} F_{r}^{2}$ is the corresponding Casimir operator, which acts on $\mathcal{H}$ as a multiple $c \mathbf{1}$ of the identity, one easily finds that

$$
(\Delta F)^{2}=c-\left\|\Phi_{\pi}([v])\right\|^{2},
$$

which is minimal if $\left\|\Phi_{\pi}([v])\right\|$ is maximal. As $I_{\pi}=\operatorname{conv}\left(\mathcal{O}_{\pi}\right)$ holds for a coadjoint orbit $\mathcal{O}_{\pi}$, and the scalar product on $\mathfrak{g}$, resp., $\mathfrak{g}^{\prime}$ is invariant, the orbit $\mathcal{O}_{\pi}$ is contained in a sphere. Therefore the invariant dispersion $(\Delta F)^{2}$ is minimal in a state $[v]$ if and only if $\Phi_{\pi}([v]) \in \mathcal{O}_{\pi}=\operatorname{Ext}\left(I_{\pi}\right)$.

\section{Invariant Cones in Lie Algebras}

In this section we take a closer look at important examples of invariant convex cones in Lie algebras.

\subsection{Invariant cones in unitary Lie algebras}

Examples 6.1. If $\mathcal{H}$ is a complex Hilbert space, then the Lie algebra $\mathfrak{u}(\mathcal{H})$ of skew-hermitian bounded operators contains the open invariant cone

$$
C_{\mathfrak{u}(\mathcal{H})}:=\{x \in \mathfrak{u}(\mathcal{H}): i x \ll 0\}
$$

(cf. Example 4.10).

More generally, for any unital $C^{*}$-algebra $\mathcal{A}$, the Banach-Lie algebra $\mathfrak{u}(\mathcal{A})=$ $\left\{x \in \mathcal{A}: x^{*}=-x\right\}$ contains the open invariant cone

$$
C_{\mathfrak{u}(\mathcal{A})}:=\{x \in \mathfrak{u}(\mathcal{A}): i x \ll 0\} .
$$

\subsection{Invariant cones in symplectic Lie algebras}

Definition 6.2. If $V$ is a Banach space and $q: V \rightarrow \mathbb{R}$ a continuous quadratic form, then we say that $q$ is strongly positive definite, written $q \gg 0$, if $\sqrt{q}$ defines a Hilbert norm on $V$. In particular, we are asking for $V$ to be complete with respect to this norm.

Now let $(V, \omega)$ be a strongly symplectic Banach space (cf. Remark B.2) and $\mathfrak{s p}(V, \omega) \subseteq \mathfrak{g l}(V)$ be the corresponding symplectic Lie algebra. We associate to each $X \in \mathfrak{s p}(V, \omega)$ the quadratic Hamiltonian $H_{X}(v):=\frac{1}{2} \omega(X v, v)$ and obtain an open invariant cone by

$$
W_{\mathfrak{s p}(V, \omega)}:=\left\{X \in \mathfrak{s p}(V, \omega): H_{X} \gg 0\right\}
$$

which is non-empty if and only if $V$ is topologically isomorphic to a real Hilbert space which carries a complex Hilbert space structure $\langle\cdot, \cdot\rangle$ with

$$
\omega(v, w)=\operatorname{Im}\langle v, w\rangle \quad \text { for } v, w \in V
$$

(cf. Proposition B.3, [1, Theorem 3.1.19]). 
Definition 6.3. Actually $\mathfrak{s p}(V, \omega)$ is a Lie subalgebra of the semidirect product

$$
\mathfrak{h} \mathfrak{s p}(V, \omega):=\mathfrak{h e i s}(V, \omega) \rtimes \mathfrak{s p}(V, \omega),
$$

where $\mathfrak{h e i s}(V, \omega)=\mathbb{R} \oplus_{\omega} V$ is the Heisenberg algebra associated to $(V, \omega)$, with the bracket

$$
\left[(z, v),\left(z^{\prime}, v^{\prime}\right)\right]:=\left(\omega\left(v, v^{\prime}\right), 0\right)
$$

Since every continuous linear functional on $V$ is of the form $i_{x} \omega$, the discussion in Remark B.2 implies that $\mathfrak{h} \mathfrak{s p}(V, \omega)$ can be identified with the space of continuous polynomials of degree $\leq 2$ on $V$, endowed with the Poisson bracket

$$
\{f, g\}=\omega\left(X_{g}, X_{f}\right) \quad \text { where } i_{X_{f}} \omega=\mathrm{d} f .
$$

Let Heis $(V, \omega):=\mathbb{R} \times V$ be the Heisenberg group of $(V, \omega)$ with the multiplication

$$
(t, v)\left(t^{\prime}, v^{\prime}\right):=\left(t+t^{\prime}+\frac{1}{2} \omega\left(v, v^{\prime}\right), v+v^{\prime}\right) .
$$

Then the Jacobi group $\operatorname{HSp}(V, \omega):=\operatorname{Heis}(V, \omega) \rtimes \operatorname{Sp}(V, \omega)$ acts by $\sigma((c, w, g), v):=$ $w+g v$ on $V$ and the corresponding derived action is given by $\dot{\sigma}(z, w, x)(v)=$ $-w-X v$, so that

$$
\left(i_{\dot{\sigma}(z, w, x)} \omega\right)_{v}=-\omega(w+X v, \cdot)=-i_{w+X v} \omega .
$$

Therefore this action is Hamiltonian with equivariant momentum map

$$
\Phi: \mathcal{H} \rightarrow \mathfrak{h} \mathfrak{s p}(V, \omega)^{\prime}, \quad \Phi(v)(c, w, A):=-c-\omega(w, v)-\frac{1}{2} \omega(A v, v)
$$

(cf. [60, Proposition A.IV.15], where we use a different sign convention).

Lemma 6.4. The convex cone

$$
W_{\mathfrak{h} \mathfrak{s p}(V, \omega)}^{+}:=\left\{(c, v, A) \in \mathfrak{h} \mathfrak{s p}(V, \omega):(\forall v \in V) c+\omega(x, v)+H_{A}(v)>0, H_{A} \gg 0\right\}
$$

is open and invariant in the Banach-Lie algebra $\mathfrak{h} \mathfrak{s p}(V, \omega)$. It is contained in the larger open invariant cone

$$
W_{\mathfrak{h} \mathfrak{s p}(V, \omega)}:=\left\{(c, v, A) \in \mathfrak{h} \mathfrak{s p}(V, \omega): H_{A} \gg 0\right\}
$$

Proof. It is clear that $W:=W_{\mathfrak{h} \mathfrak{s}(V, \omega)}^{+}$is an invariant convex cone. It remains to show that it is open. If $f(v)=c+\omega(x, v)+\frac{1}{2} \omega(A v, v)$ is such that $H_{A}$ is strictly positive, then $\mathrm{d} f(v)=i_{x} \omega+i_{A v} \omega$, which vanishes if and only if $v=-A^{-1} x$. It follows in particular, that each such function has a unique minimal value which is given by

$$
f\left(-A^{-1} x\right)=c-\omega\left(x, A^{-1} x\right)+\frac{1}{2} \omega\left(x, A^{-1} x\right)=c-\frac{1}{2} \omega\left(x, A^{-1} x\right) .
$$

Therefore the condition $f>0$ is equivalent to $c>\frac{1}{2} \omega\left(x, A^{-1} x\right)$, showing that $W$ is indeed open in $\mathfrak{h} \mathfrak{s p}(V, \omega)$. 
From now on we assume that $\mathcal{H}$ is a complex Hilbert space, $V=\mathcal{H}_{\mathbb{R}}$ is the underlying real Banach space, and $\omega(v, w):=\operatorname{Im}\langle v, w\rangle$ is the corresponding symplectic form. Then $I v=i v$ is a complex structure on $\mathcal{H}_{\mathbb{R}}$ leaving $\omega$ invariant. It satisfies

$$
\omega(I v, v)=\operatorname{Im}\langle i v, v\rangle=\|v\|^{2} .
$$

Formalizing this property leads to:

Definition 6.5. We call a real linear complex structure $J: \mathcal{H} \rightarrow \mathcal{H} \omega$-positive if $\omega(J v, w)$ is symmetric and positive definite and write $\mathcal{I}_{\omega}$ for the set of $\omega$-positive complex structures on $\mathcal{H}$.

The following lemma is well known for the finite dimensional case, but it carries over to infinite dimensional Hilbert spaces. It implies in particular that $\mathcal{I}_{\omega}=\mathcal{O}_{I}$ is an adjoint orbit of the Lie algebra $\mathfrak{s p}(\mathcal{H})$.

Lemma 6.6. The following assertions hold:

(i) $\operatorname{Sp}(\mathcal{H}) \cap \mathfrak{s p}(\mathcal{H})=\left\{g \in \operatorname{GL}\left(\mathcal{H}_{\mathbb{R}}\right): I g^{\top} I=-g^{-1}=g\right\}$ is the set of complex structures $J$ on $\mathcal{H}$ for which $\omega(J v, w)$ is symmetric.

(ii) $\mathcal{I}_{\omega}=I e^{\mathfrak{p}}$ for $\mathfrak{p}:=\{x \in \mathfrak{s p}(\mathcal{H}): I x=-x I\}=\left\{x \in \mathfrak{s p}(\mathcal{H}): x^{\top}=x\right\}$.

(iii) The conjugation action of $\operatorname{Sp}(\mathcal{H})$ on $\mathcal{I}_{\omega}$ leads to a diffeomorphism $\mathcal{I}_{\omega} \cong$ $\operatorname{Sp}(\mathcal{H}) / \mathrm{U}(\mathcal{H})$.

(iv) If $A \in \mathfrak{s p}(\mathcal{H})$ is such that $H_{A} \gg 0$, then there exists a unique $\omega$-positive complex structure $J$ on $\mathcal{H}$ commuting with $A$.

(v) $\mathcal{I}_{\omega}^{\text {res }}:=\operatorname{Ad}\left(\operatorname{Sp}_{\text {res }}(\mathcal{H})\right) I=\left\{J \in \mathcal{I}_{\omega}:\|I-J\|_{2}<\infty\right\}=\mathcal{I}_{\omega} \cap \mathfrak{s p}_{\text {res }}(\mathcal{H})$.

Proof. (i) That $\omega(J v, w)$ is symmetric is equivalent to $J \in \mathfrak{s p}(\mathcal{H})$, and as $J^{-1}=$ $-J$ characterizes complex structures, (i) follows.

(ii) We have seen in (13) that $I$ is $\omega$-positive. Let $J$ be another $\omega$-positive complex structure. Writing $J=u e^{x}$ according to the polar decomposition of $\operatorname{Sp}(\mathcal{H})$ with $u \in \mathrm{U}(\mathcal{H})$ and $x \in \mathfrak{p}\left(\left[63\right.\right.$, Theorem I.6(iv)]), we see that $J^{2}=-\mathbf{1}$ is equivalent to $u^{2}=-\mathbf{1}(u$ is a complex structure) and $u x=-x u$ ( $x$ is antilinear with respect to $u$ ). If this is the case, then

$$
\omega(J v, v)=\omega\left(u e^{x} v, v\right)=\omega\left(e^{-x / 2} u e^{x / 2} v, v\right)=\omega\left(u e^{x / 2} v, e^{x / 2} v\right)
$$

shows that $J$ is $\omega$-positive if and only if $u$ has this property. Since $u$ is complex linear, $\mathcal{H}$ decomposes into $\pm i$-eigenspaces $\mathcal{H}_{ \pm}$of $u$. Then $\omega(u v, v)$ is positive definite on $\mathcal{H}_{+}$and negative definite on $\mathcal{H}_{-}$, so that the $\omega$-positivity implies $u=I$. This proves (ii).

(iii) For $g=u e^{x} \in \operatorname{Sp}(\mathcal{H})$, the relation $g^{-1} I g=e^{-x} I e^{x}=I e^{2 x}$ shows that $\operatorname{Sp}(\mathcal{H})$ acts transitively on $\mathcal{I}_{\omega}$. As $\mathrm{U}(\mathcal{H})$ is the stabilizer of $I$, (iii) follows from the smoothness of the map $J=I e^{x} \mapsto x=\frac{1}{2} \log \left(J^{\top} J\right)$. 
(iv) Let $(v, w)_{A}:=\omega(A v, w)$ denote the real Hilbert structure on $\mathcal{H}$ defined by A. Then

$$
\omega(x, y)=\omega\left(A\left(A^{-1} x\right), y\right)=\left(A^{-1} x, y\right)_{A}
$$

implies that $A^{-1}$ is skew-symmetric with respect to $(\cdot, \cdot)_{A}$, and the same holds for $A$ itself. Therefore $-A^{2} \geq 0$ and $J:=\left(-A^{2}\right)^{-1 / 2} A$ is a complex structure leaving $(\cdot, \cdot)_{A}$ invariant (cf. [1, Theorem 3.1.19]). Then

$$
\omega(J v, v)=\omega\left(\left(-A^{2}\right)^{-1 / 2} A v, v\right)=\left(\left(-A^{2}\right)^{-1 / 2} v, v\right)_{A}
$$

is positive definite, so that $J$ is $\omega$-positive.

If $J^{\prime}$ is another $\omega$-positive complex structure commuting with $A$, then the construction of $J$ shows that it also commutes with $J$. Therefore $J J^{\prime}$ is an involution and since $J$ and $J^{\prime}$ are $\omega$-positive, the -1-eigenspace of this involution is trivial, which leads to $J^{\prime}=J$.

(v) From the polar decomposition

$$
\operatorname{Sp}_{\text {res }}(\mathcal{H})=\mathrm{U}(\mathcal{H}) \exp \left(\mathfrak{p}_{2}\right) \quad \text { with } \mathfrak{p}_{2}:=\mathfrak{p} \cap \mathfrak{s p}_{\text {res }}(\mathcal{H})=\left\{x \in \mathfrak{p}:\|x\|_{2}<\infty\right\}
$$

we derive that $\mathcal{I}_{\omega}^{\text {res }}=I e^{\mathfrak{p}_{2}}$. For the entire function $F(z):=\frac{e^{z}-1}{z}$, we then have

$$
I e^{x}-I=I F(x) x .
$$

Since $F(x)$ is invertible for the real symmetric operator $x$ by the Spectral Mapping Theorem, it follows that $I e^{x}-I$ is Hilbert-Schmidt if and only if $x$ has this property.

For $J=I e^{x}$ we also observe that

$$
[I, J]=-e^{x}-I e^{x} I=e^{-x}-e^{x}=e^{-x}\left(\mathbf{1}-e^{2 x}\right)=-e^{-x} F(2 x) 2 x,
$$

so that $[I, J]$ is Hilbert-Schmidt if and only if $x$ is. This leads to $\mathcal{I}_{\omega}^{\text {res }}=\mathcal{I}_{\omega} \cap \mathfrak{s p}$ res $(\mathcal{H})$.

The following theorem is a useful tool when dealing with invariant cones in symplectic Lie algebras.

Theorem 6.7. For the canonical open invariant cones in $\mathfrak{s p}(\mathcal{H}), \mathfrak{s p}_{\text {res }}(\mathcal{H})$ and $\mathfrak{h} \mathfrak{s p}(\mathcal{H})$ we have the following conjugacy results:

(i) $W_{\mathfrak{s p}(\mathcal{H})}=\operatorname{Ad}(\operatorname{Sp}(\mathcal{H})) C_{\mathfrak{u}(\mathcal{H})}$ and $C_{\mathfrak{u}(\mathcal{H})}=W_{\mathfrak{s p}(\mathcal{H})} \cap \mathfrak{u}(\mathcal{H})$.

(ii) $W_{\mathfrak{s p}}(\mathcal{H}):=W_{\mathfrak{s p}(\mathcal{H})} \cap \mathfrak{s p}_{\text {res }}(\mathcal{H})=\operatorname{Ad}\left(\operatorname{Sp}_{\text {res }}(\mathcal{H})\right) C_{\mathfrak{u}(\mathcal{H})}$.

(iii) $W_{\mathfrak{h} \mathfrak{s p}(\mathcal{H})}=\operatorname{Ad}(\operatorname{HSp}(\mathcal{H}))\left(\mathbb{R} \times\{0\} \times C_{\mathfrak{u}(\mathcal{H})}\right)$ for the corresponding Lie group $\operatorname{HSp}(\mathcal{H})=\operatorname{Heis}(\mathcal{H}) \rtimes \operatorname{Sp}(\mathcal{H})$.

(iv) $W_{\mathfrak{h} \mathfrak{p p}_{\text {res }}(\mathcal{H})}:=W_{\mathfrak{h} \mathfrak{s p}(\mathcal{H})} \cap \mathfrak{h s p}_{\text {res }}(\mathcal{H})=\operatorname{Ad}\left(\operatorname{HSp}_{\text {res }}(\mathcal{H})\right)\left(\mathbb{R} \times\{0\} \times C_{\mathfrak{u}(\mathcal{H})}\right)$ for $\operatorname{HSp}_{\text {res }}(\mathcal{H}):=\operatorname{Heis}(\mathcal{H}) \rtimes \operatorname{Sp}_{\text {res }}(\mathcal{H})$.

Proof. (i) If $A \in \mathfrak{s p}(\mathcal{H})$ is complex linear, then $A^{*}=-A$, so that $\omega(A v, v)=$ $\operatorname{Im}\langle A v, v\rangle=\langle-i A v, v\rangle$. Therefore $A \in W_{\mathfrak{s p}(\mathcal{H})}$ is equivalent to $i A \ll 0$, i.e. $W_{\mathfrak{s p}(\mathcal{H})} \cap \mathfrak{u}(\mathcal{H})=C_{\mathfrak{u}(\mathcal{H})}$. 
For $A \in W_{\mathfrak{s p}(\mathcal{H})}$ we find with Lemma $6.6(\mathrm{iv})$ a $J \in \mathcal{I}_{\omega}$ commuting with $A$. For any $g \in \operatorname{Sp}(\mathcal{H})$ with $J=\operatorname{Ad}(g) I$, whose existence follows from Lemma 6.6(iii), we conclude that $\operatorname{Ad}(g)^{-1} A$ commutes with $I$, hence is contained in $W_{\mathfrak{s p}(\mathcal{H})} \cap \mathfrak{u}(\mathcal{H})=$ $C_{\mathfrak{u}(\mathcal{H})}$. This proves (i).

(ii) In view of Lemma $6.6(\mathrm{v})$, it suffices to show that for any $A \in W_{\mathfrak{s p}_{\text {res }}(\mathcal{H})}$ the corresponding $\omega$-positive complex structure $J=\left(-A^{2}\right)^{-1 / 2} A$ from Lemma 6.6(iv) is contained in $\mathcal{I}_{\omega}^{\text {res }}$, i.e. $[I, J]$ is Hilbert-Schmidt. Since $A^{2}$ commutes with $I$, we have $[I, J]=\left(-A^{2}\right)^{-1 / 2}[I, A]$, so that the invertibility of $\left(-A^{2}\right)^{-1 / 2}$ implies that $A \in \mathfrak{s p}_{\text {res }}(\mathcal{H})$ is equivalent to $J \in \mathfrak{s p}_{\text {res }}(\mathcal{H})$.

(iii) As we have seen in the proof of Lemma 6.4 above, for each element $(c, x, A) \in W_{\mathfrak{h} \mathfrak{s p}(\mathcal{H})}$, the Hamiltonian function $f(v)=c+\omega(x, v)+H_{A}(v)$ has a unique minimum in $-A^{-1} x$. Since the adjoint action of the Heisenberg group $\operatorname{Heis}(\mathcal{H}) \subseteq \operatorname{HSp}(\mathcal{H})$ corresponds to a translation action on $\mathcal{H}$, each adjoint orbit $\mathcal{O}_{X}$ in $W_{\mathfrak{h} \mathfrak{s}(V, \omega)}$ contains an element $Y$ whose corresponding Hamiltonian function is minimal in 0 , so that $Y \in \mathbb{R} \times\{0\} \times \mathfrak{s p}(\mathcal{H})$. In view of (i), each orbit in $W_{\mathfrak{h} \mathfrak{s p}(V, \omega)}$ meets the subalgebra $\mathbb{R} \times\{0\} \times \mathfrak{u}(\mathcal{H})$, and this proves (iii).

(iv) follows by combining the argument under (iii) with the proof of (ii).

\subsection{Invariant Lorentzian cones}

If $(\mathfrak{g}, \beta)$ is a Lorentzian Lie algebra, i.e. $\beta$ is an invariant Lorentzian form (which is negative definite on a closed hyperplane), then each half of the open double cone $\{x \in \mathfrak{g}: \beta(x, x)>0\}$ is an open invariant cone in $\mathfrak{g}$.

Example 6.8. A particularly important example is $\mathfrak{g}=\mathfrak{s l}_{2}(\mathbb{R})$ with $\beta(x, y)=$ $-\operatorname{tr}(x y)$. Indeed, the basis

$$
h=\left(\begin{array}{rr}
1 & 0 \\
0 & -1
\end{array}\right), \quad u=\left(\begin{array}{rr}
0 & 1 \\
-1 & 0
\end{array}\right), \quad t=\left(\begin{array}{ll}
0 & 1 \\
1 & 0
\end{array}\right)
$$

is orthogonal with

$$
\beta(x h+y u+z t, x h+y u+z t)=-2 x^{2}+2 y^{2}-2 z^{2} .
$$

For $\mathfrak{g}=\mathfrak{s l}_{2}(\mathbb{R})$, the adjoint group coincides with the identity component $\operatorname{SO}(\mathfrak{g}, \beta)_{0} \cong$ $\mathrm{SO}_{1,2}(\mathbb{R})_{0}$, which implies that the adjoint and coadjoint orbits are the connected components of the level surfaces of the associated quadratic form, and the 0-level surface of isotropic vectors decomposes into the 0-orbit and two isotropic orbits lying in the boundary of the double cone. This description of the adjoint orbits implies in particular that there are precisely two nontrivial open invariant cones, namely the connected components of the set $\{x: \beta(x, x)>0\}$.

Remark 6.9. Other examples of Lorentzian Lie algebras arise as double extensions from Lie algebras $\mathfrak{g}^{0}$, endowed with an invariant scalar product $\kappa^{0}:$ If $D \in \operatorname{der}\left(\mathfrak{g}^{0}, \kappa^{0}\right)$ is a continuous skew-symmetric derivation, then $\mathfrak{g}:=\mathbb{R} \times \mathfrak{g}^{0} \times \mathbb{R}$ is a Lie algebra with respect to the bracket

$$
\left[(z, x, t),\left(z^{\prime}, x^{\prime}, t^{\prime}\right)\right]=\left(\kappa^{0}\left(D x, x^{\prime}\right), t D x^{\prime}-t^{\prime} D x+\left[x, x^{\prime}\right], 0\right)
$$


and the continuous symmetric bilinear form

$$
\kappa\left((z, x, t),\left(z^{\prime}, x^{\prime}, t^{\prime}\right)\right):=z t^{\prime}+z^{\prime} t+\kappa^{0}\left(x, x^{\prime}\right),
$$

is easily seen to be invariant. The pair $(\mathfrak{g}, \kappa)$ is called a double extension of $\left(\mathfrak{g}^{0}, \kappa^{0}\right)$ (cf. [54]).

Example 6.10. (a) If $\mathfrak{k}$ is a compact Lie algebra and $\mathfrak{g}^{0}:=C^{\infty}\left(\mathbb{S}^{1}, \mathfrak{k}\right)$ is the corresponding loop algebra, then we identify its elements with $2 \pi$-periodic functions on $\mathbb{R}$. With an invariant scalar product $\kappa_{\mathfrak{k}}$ on $\mathfrak{k}$, we obtain the invariant scalar product

$$
\kappa^{0}(\xi, \eta):=\int_{0}^{2 \pi} \kappa_{\mathfrak{k}}(\xi(t), \eta(t)) d t
$$

on $\mathfrak{g}^{0}$, and the derivation $D \xi:=\xi^{\prime}$ is skew-symmetric. The corresponding double extension produces the (unitary forms) of the untwisted affine Kac-Moody Lie algebras.

(b) If $\mathfrak{g}^{0}:=\mathfrak{u}_{2}(\mathcal{H})$ is the Lie algebra of skew-hermitian Hilbert-Schmidt operators on the Hilbert space $\mathcal{H}$ and $A=-A^{*} \in \mathfrak{u}(\mathcal{H})$, then $\kappa^{0}(x, y):=\operatorname{tr}\left(x y^{*}\right)=$ $-\operatorname{tr}(x y)$ is an invariant scalar product on $\mathfrak{g}^{0}$ and one obtains a double extension for the derivation $D(x):=[A, x]$. It is nontrivial if and only if $A \notin \mathbb{R} i \mathbf{1}+\mathfrak{u}_{2}(\mathcal{H})$ (cf. [63]).

Problem 6.11. Classify infinite dimensional Lorentzian Lie algebras $\mathfrak{g}$ which are complete in the sense that for $x \in \mathfrak{g}$ with $\beta(x, x)>0$ the orthogonal space is a Hilbert space with respect to $-\beta$. The construction in Example 6.10(b) produces interesting examples.

Finite dimensional indecomposable Lorentzian Lie algebras have been classified by Hilgert and Hofmann in [35]. The simple result is that an indecomposable finite dimensional Lorentzian Lie algebra is either $\mathfrak{s l}_{2}(\mathbb{R})$, endowed with the negative of its Cartan-Killing form, or a double extension of an Abelian Lie algebra, defined by an invertible skew-symmetric derivation $D$ ([36, Theorem II.6.14]).

\subsection{Invariant cones of vector fields}

Example 6.12. Let $\mathfrak{g}=\mathcal{V}\left(\mathbb{S}^{1}\right)=C^{\infty}\left(\mathbb{S}^{1}\right) \partial_{\theta}$ be the Lie algebra of smooth vector fields on the circle $\mathbb{S}^{1} \cong \mathbb{R} / \mathbb{Z}$, where $\partial_{\theta}:=\frac{\partial}{\partial \theta}$ denotes the generator of the right rotations. Then

$$
W_{\mathcal{V}\left(\mathbb{S}^{1}\right)}:=\left\{f \partial_{\theta}: f>0\right\}
$$

is an open invariant cone (cf. Sec. 7).

Example 6.13. The preceding example has a natural higher dimensional generalization. Let $(M, g)$ be a compact Lorentzian manifold possessing a timelike vector field $T$, i.e. $g_{m}(T(m), T(m))>0$ for every $m \in M$. Then

$$
W:=\{X \in \mathcal{V}(M):(\forall m \in M) g(X, X)>0, g(X, T)>0\}
$$


is an open convex cone in the Fréchet space $\mathcal{V}(M)$ and its intersection with the subalgebra $\operatorname{conf}(M, g)$ of conformal vector fields is an open invariant cone. For $M=\mathbb{S}^{1}$ all vector fields are conformal and we thus obtain Example 6.12.

\subsection{Invariant cones and symmetric Hilbert domains}

Example 6.14. (a) We recall from Example 3.2(g) the concept of a symmetric Hilbert domain and write $G:=\operatorname{Aut}(\mathcal{D})_{0}$ for the identity component of its automorphism group. We assume w.l.o.g. that $\mathcal{D}$ is the open unit ball of a complex Banach space $V$ (cf. [42,43]). Let $K=G \cap \mathrm{GL}(V)$ be the subgroup of linear automorphisms of $\mathcal{D}$, i.e. the group of complex linear isometries of $V$ and let $\mathfrak{k}=\mathbf{L}(K)$ be its Lie algebra. Then

$$
W_{\mathfrak{k}}:=\left\{x \in \mathfrak{k}:\left\|e^{i x}\right\|<1\right\}
$$

is a pointed open convex cone in the subalgebra $\mathfrak{k}$, and

$$
W:=\operatorname{Ad}(G) W_{\mathfrak{k}}
$$

is a pointed open invariant convex cone in $\mathfrak{g}$ (cf. [61, Theorem V.9], and [105, Theorem 5] for the finite dimensional case). By definition, this open cone has the interesting property that every orbit in $W$ meets $\mathfrak{k}$.

(b) Because they will also show up in the following, we take a closer look at some characteristic examples. In Examples 3.2(g), we have seen that the group $G=\mathrm{U}_{\text {res }}\left(\mathcal{H}_{+}, \mathcal{H}_{-}\right)$acts naturally on the circular symmetric Hilbert domain $\mathcal{D}:=$ $\left\{z \in B_{2}\left(\mathcal{H}_{+}, \mathcal{H}_{-}\right):\|z\|<1\right\}$. Here the stabilizer of 0 is

$$
K:=\mathrm{U}\left(\mathcal{H}_{-}\right) \times \mathrm{U}\left(\mathcal{H}_{+}\right),
$$

which acts by $(a, d) z=a z d^{-1}$. From the Hilbert space isomorphism $B_{2}\left(\mathcal{H}_{+}, \mathcal{H}_{-}\right) \cong$ $\mathcal{H}_{-} \widehat{\otimes} \mathcal{H}_{+}^{*}$, one now derives with

$$
\begin{aligned}
\left\|e^{i x_{-}} \otimes e^{-i x_{+}}\right\| & =\left\|e^{i x_{-}}\right\|\left\|e^{-i x_{+}}\right\|=\sup \operatorname{Spec}\left(i x_{-}\right)+\sup \operatorname{Spec}\left(-i x_{+}\right) \\
& =\sup \operatorname{Spec}\left(i x_{-}\right)-\inf \operatorname{Spec}\left(i x_{+}\right)
\end{aligned}
$$

that

$$
W_{\mathfrak{k}}=\left\{\left(x_{-}, x_{+}\right) \in \mathfrak{k} \cong \mathfrak{u}\left(\mathcal{H}_{-}\right) \times \mathfrak{u}\left(\mathcal{H}_{+}\right): \sup \operatorname{Spec}\left(i x_{-}\right)<\inf \operatorname{Spec}\left(i x_{+}\right)\right\},
$$

and $W=\operatorname{Ad}(G) W_{\mathfrak{k}}$ is the corresponding open invariant cone in $\mathfrak{g}=\mathfrak{u}\left(\mathcal{H}_{+}, \mathcal{H}_{-}\right)$. Since the action of $G$ on $\mathcal{D}$ is not faithful, this cone has a nontrivial edge $H(W)=$ $\mathbb{R} i \mathbf{1}=\mathbf{L}(\mathbb{T} \mathbf{1})$.

(c) For $\mathcal{H}_{+}=\mathcal{H}_{-}=\mathcal{H}$ and the subgroup $G=\operatorname{Sp}_{\text {res }}(\mathcal{H}) \subseteq \mathrm{U}_{\text {res }}\left(\mathcal{H}_{+}, \mathcal{H}_{-}\right)$, we have $K \cong \mathrm{U}(\mathcal{H})$, corresponding to the pairs of the form $\left(a, a^{-\top}\right) \in \mathrm{U}(\mathcal{H}) \times \mathrm{U}(\mathcal{H})$, and, accordingly,

$$
W_{\mathfrak{k}}=\{x \in \mathfrak{u}(\mathcal{H}) i x \ll 0\}=C_{\mathfrak{u}(\mathcal{H})}
$$

(Examples 3.2(h) and 6.1). From Theorem 6.7(ii) it now follows that

$$
W=\operatorname{Ad}(G) W_{\mathfrak{k}}=W_{\mathfrak{s p}_{\text {res }}}(\mathcal{H}) .
$$




\subsection{A general lemma}

The following lemma captures the spirit of some arguments in the previous constructions of invariant convex cones in Lie algebras in a quite natural way. F.i., it applies to finite dimensional simple algebras as well as $\mathcal{V}\left(\mathbb{S}^{1}\right)$.

Lemma 6.15. Suppose that the element $d \in \mathfrak{g}$ has the following properties:

(a) The interior $W_{\min }$ of the invariant convex cone generated by $\mathcal{O}_{d}=\operatorname{Ad}(G) d$ is non-empty and different from $\mathfrak{g}$.

(b) There exists a continuous linear projection $p: \mathfrak{g} \rightarrow \mathbb{R} d$ which preserves every open and closed convex subset.

(c) There exists an element $x \in \mathfrak{g}$ for which $p\left(\mathcal{O}_{x}\right)$ is unbounded.

Then each non-empty open invariant cone $W$ contains $W_{\min }$ or $-W_{\min }$, and for $\lambda \in \mathfrak{g}^{\prime}$ the following are equivalent

(i) $\lambda \in \mathfrak{g}_{\mathrm{seq}}^{\prime}$, i.e. $\mathcal{O}_{\lambda}$ is semi-equicontinuous.

(ii) $\mathcal{O}_{\lambda}(d)$ is bounded from below or above.

(iii) $\lambda \in W_{\min }^{\star} \cup-W_{\min }^{\star}$.

Proof. If $W \subseteq \mathfrak{g}$ is an open invariant cone, then $p(W) \subseteq \mathbb{R} d$ contains an open half line, so that (b) implies that $W$ contains either $d$ or $-d$. Accordingly, we then have $W_{\text {min }} \subseteq W$ or $-W_{\text {min }} \subseteq W$.

(iii) $\Rightarrow$ (i): As $W_{\min }$ is open, $W_{\min }^{\star} \cup-W_{\text {min }}^{\star}$ consists of semi-equicontinuous coadjoint orbits (Example 2.5(b)).

(i) $\Rightarrow$ (ii): Let $\lambda \in \mathfrak{g}_{\text {seq }}^{\prime}$. Since $B\left(\mathcal{O}_{\lambda}\right)^{0}$ is an open invariant cone, (a) implies that it either contains $d$ or $-d$, which is (ii).

(ii) $\Rightarrow$ (iii): Assume w.l.o.g. that $\inf \mathcal{O}_{\lambda}(d)>-\infty$. We claim that $\mathcal{O}_{\lambda} \subseteq W_{\text {min }}^{\star}$, which is equivalent to $\mathcal{O}_{\lambda}(d) \geq 0$.

To this end, we consider $\mu \in \mathfrak{g}^{\prime}$ defined by $p(x)=\mu(x) d$. Then $-d \notin W_{\text {min }}$ and (b) imply that $\mu \in W_{\text {min }}^{\star}$, so that $\mu\left(\mathcal{O}_{d}\right) \geq 0$. If $\mu\left(\mathcal{O}_{d}\right)$ is bounded from above, then $\pm d \in B\left(\mathcal{O}_{\mu}\right)$, so that the invariance of the convex cone $B\left(\mathcal{O}_{\mu}\right)$ leads to $\pm W_{\min } \subseteq B\left(\mathcal{O}_{\mu}\right)$, and hence to $\mathfrak{g}=B\left(\mathcal{O}_{\mu}\right)$, contradicting (c). We conclude that $\mu\left(\mathcal{O}_{d}\right)$ is unbounded. Applying (b) to the closed convex subset $C:=\overline{\operatorname{conv}}\left(\mathcal{O}_{d}\right)$, it follows that $[1, \infty[\cdot d \subseteq C$, and hence that $d \in \lim (C)$ (Lemma 2.9(iii)). As $\lim (C)$ is an invariant cone, (a) entails $W_{\min } \subseteq \lim (C)$. We finally conclude that $\lambda \in B\left(\mathcal{O}_{d}\right)=B(C) \subseteq \lim (C)^{\star} \subseteq W_{\min }^{\star}($ Lemma $2.9($ vi $))$.

The preceding lemma applies in particular to finite dimensional simple noncompact Lie algebras ([105]):

Proposition 6.16. For a finite dimensional simple non-compact Lie algebra $\mathfrak{g}$, the following assertions hold:

(i) Every non-empty invariant convex subset $C \neq\{0\}$ has interior points.

(ii) If $\mathfrak{g}$ contains a proper open invariant convex cone, then there exist minimal and maximal open invariant cones $W_{\min } \subseteq W_{\max }$ such that for any other open 
invariant cone $W$ we either have

$$
W_{\min } \subseteq W \subseteq W_{\max } \quad \text { or } \quad W_{\min } \subseteq-W \subseteq W_{\max } .
$$

In this case the set of semi-equicontinuous coadjoint orbit in $\mathfrak{g}^{\prime}$ coincides with $W_{\min }^{\star} \cup-W_{\min }^{\star}$.

Proof. (i) If $\emptyset \neq C \neq\{0\}$ is invariant and convex, then $\operatorname{span}(C)$ is a nonzero ideal of $\mathfrak{g}$, hence equal to $\mathfrak{g}$. If the affine subspace generated by $C$ is proper, then its translation space is a hyperplane ideal of $\mathfrak{g}$, contradicting the simplicity of $\mathfrak{g}$. Therefore $C$ generates $\mathfrak{g}$ as an affine space, hence has interior points because $\operatorname{dim} \mathfrak{g}<\infty$ and $C$ contains a simplex of maximal dimension.

(ii) Let $\mathfrak{g}=\mathfrak{k} \oplus \mathfrak{p}$ be a Cartan decomposition of $\mathfrak{g}$ and $\mathfrak{z}:=\mathfrak{z}(\mathfrak{k})$ be the center of $\mathfrak{k}$. Then the existence of invariant cones implies that $\mathfrak{z}=\mathfrak{z}_{\mathfrak{g}}(\mathfrak{k})=\mathbb{R} d$ is one-dimensional ([105]). Therefore we have a fixed point projection $p_{\mathfrak{z}}: \mathfrak{g} \rightarrow \mathfrak{z}$ with respect to the action of the compact subgroup $e^{\text {ad } \mathfrak{k}}$, and this projection preserves all open and closed invariant convex subsets (Proposition 2.11(a)). If $p_{\mathfrak{z}}\left(\mathcal{O}_{x}\right)$ is bounded for every $x \in \mathfrak{g}$, then $p_{\mathfrak{z}}$, considered as a linear functional on $\mathfrak{g}$, has a bounded orbit. As $\mathfrak{g}$ is simple, this implies that all coadjoint orbits are bounded, and this contradicts the non-compactness of $\mathfrak{g}$. Therefore (a)-(c) in Lemma 6.15 are satisfied, which implies that the set of semi-equicontinuous coadjoint orbits is $W_{\min }^{\star} \cup-W_{\min }^{\star}$.

\section{Connections to $C^{*}$-Algebras}

In this section we discuss two aspects of semibounded representation theory in the context of $C^{*}$-algebras. The first one concerns the momentum sets of restrictions of representations of a unital $C^{*}$-algebra $\mathcal{A}$ to its unitary group $\mathrm{U}(\mathcal{A})$, and the second one concerns covariant representations for $C^{*}$-dynamical systems defined by a Banach-Lie group $H$ acting on a $C^{*}$-algebra $\mathcal{A}$. Using the results from Appendix A, it follows that covariant representations lead to smooth unitary representations of the Lie group $\mathrm{U}\left(\mathcal{A}^{\infty}\right) \rtimes H$, so that spectral conditions for covariant representations can be interpreted in terms of semibounded representations and the theory of invariant cones in Lie algebras becomes available.

In this subsection $\mathcal{A}$ denotes a unital $C^{*}$-algebra and $\mathrm{U}(\mathcal{A})$ its unitary group, considered as a Banach-Lie group (Example 3.2(a)). We identify the state space

$$
S(\mathcal{A}):=\left\{\varphi \in \mathcal{A}^{\prime}: \varphi(\mathbf{1})=\|\varphi\|=1\right\} \subseteq\left\{\varphi \in \mathcal{A}^{\prime}: \varphi(\mathfrak{u}(\mathcal{A})) \subseteq i \mathbb{R}\right\}
$$

of $\mathcal{A}$ with a subset of $\mathfrak{u}(\mathcal{A})^{\prime}$ by mapping $\varphi \in S(\mathcal{A})$ to the real-valued functional $-\left.i \varphi\right|_{\mathfrak{u}(\mathcal{A})} \in \mathfrak{u}(\mathcal{A})^{\prime}$ (cf. [60, Sec. X.5] for more details).

\subsection{Representations of the unitary group}

If $(\pi, \mathcal{H})$ is a unitary representation of $\mathrm{U}(\mathcal{A})$ obtained by restricting an algebra representation, then its momentum set is simply given by

$$
I_{\pi}=\{\varphi \in S(\mathcal{A}): \varphi(\operatorname{ker} \pi)=\{0\}\}=S(\mathcal{A}) \cap(\operatorname{ker} \pi)^{\perp} \cong S(\pi(\mathcal{A}))
$$


(cf. [60, Theorem X.5.13]). In particular, the momentum set is completely determined by the kernel of the representation $\pi$, resp., the $C^{*}$-algebra $\pi(\mathcal{A})$. This is why representations with the same kernel are called physically equivalent in algebraic Quantum Field Theory ([31]).

In $\left[20\right.$, Theorem 9.1] one finds a characterization of the separable $C^{*}$-algebras $\mathcal{A}$ of type $I$ as those for which irreducible representations are determined by their kernels, hence by their momentum sets. More generally, postliminal $C^{*}$-algebras have this property ([20, Theorem 4.3.7]). We conclude in particular that the existence of separable $C^{*}$-algebras $\mathcal{A}$ which is not of type $I$ implies the existence of nonequivalent irreducible representations $\left(\pi_{1}, \mathcal{H}_{1}\right)$ and $\left(\pi_{2}, \mathcal{H}_{2}\right)$ with $\operatorname{ker} \pi_{1}=\operatorname{ker} \pi_{2}$, and hence with $I_{\pi_{1}}=I_{\pi_{2}}$. We thus observe:

Theorem 7.1. Bounded irreducible unitary representations of Banach-Lie groups are in general not determined up to equivalence by their momentum sets.

Proposition 7.2. (Momentum sets of irreducible representations) For irreducible representations $(\pi, \mathcal{H})$ of a $C^{*}$-algebra $\mathcal{A}$, the following assertions hold:

(i) $\mathrm{U}(\mathcal{A})$ acts transitively on $\mathbb{P}(\mathcal{H})$, so that $\mathcal{O}_{\pi}:=\operatorname{im}\left(\Phi_{\pi}\right)$ is a single coadjoint orbit with $I_{\pi}=\overline{\operatorname{conv}}\left(\mathcal{O}_{\pi}\right)$.

(ii) $\mathcal{O}_{\pi}$ consists of pure states i.e. $\mathcal{O}_{\pi} \subseteq \operatorname{Ext}(S(\mathcal{A}))$.

(iii) Two irreducible representations $\pi_{1}$ and $\pi_{2}$ are equivalent if and only if $\mathcal{O}_{\pi_{1}}=\mathcal{O}_{\pi_{2}}$.

Proof. (i) [20, Theorem 2.8.3]

(ii) [20, Proposition 2.5.4]

(iii) (cf. [20, Corollary 2.8.6]) From the naturality of the momentum map it follows that equivalent representations have the same orbits. The converse follows from the fact that an irreducible representation can be recovered from any of its pure states $\varphi$ by the GNS construction, and states in the same $\mathrm{U}(\mathcal{A})$-orbit lead to equivalent representations.

In [8] these results are generalized to irreducible representations of $\mathrm{U}(\mathcal{A})$ occurring in tensor products of algebra representations and their duals.

Examples 7.3. (a) If $\pi$ is the identical representation of the $C^{*}$-algebra $\mathcal{A}=B(\mathcal{H})$ on $\mathcal{H}$, then the corresponding momentum map is given by

$$
\Phi_{\pi}([v])(x)=\frac{1}{i} \frac{\langle x v, v\rangle}{\langle v, v\rangle}=-i \operatorname{tr}\left(x P_{v}\right),
$$

where $P_{v}(w)=\frac{\langle w, v\rangle}{\langle v, v\rangle} v$ is the orthogonal projection onto $[v]$. Clearly, this representation is faithful and irreducible, so that $I_{\pi}=S(\mathcal{A})$. On the other hand, $\mathcal{O}_{\pi}=\operatorname{im}\left(\Phi_{\pi}\right)$ can be identified with the set of rank-one projections, as elements of the dual space 
$\mathcal{A}^{\prime}$. With the trace pairing, we can embed the subspace $\operatorname{Herm}_{1}(\mathcal{H})$ of hermitian trace class operators in to the dual $\mathcal{A}^{\prime}$. Then

$$
\operatorname{Herm}_{1}(\mathcal{H}) \cap S(\mathcal{A})=\left\{S \in \operatorname{Herm}_{1}(\mathcal{H}): S \geq 0, \operatorname{tr} S=1\right\}
$$

and the Spectral Theorem for compact hermitian operators implies that

$$
\operatorname{Ext}(S(\mathcal{A})) \cap \operatorname{Herm}_{1}(\mathcal{H})=\Phi_{\pi}(\mathbb{P}(\mathcal{H}))
$$

is a single coadjoint orbit. However, since the Calkin algebra $\mathcal{A} / K(\mathcal{H})$ is nontrivial if $\operatorname{dim} \mathcal{H}=\infty, \mathcal{A}$ also has pure states vanishing on the ideal $K(\mathcal{H})$ of compact operators and $\mathrm{U}(\mathcal{H})$ does not act transitively on $\operatorname{Ext}(S(\mathcal{A})$ ).

(b) For the natural representation of $G=\mathrm{U}(\mathcal{H})$ on the symmetric powers $S^{n}(\mathcal{H})$, the elements $\left[v^{n}\right], 0 \neq v \in \mathcal{H}$, form a single $G$-orbit on which the complex group $\mathrm{GL}(\mathcal{H})=\mathcal{A}^{\times}$acts holomorphically (Proposition 7.2(i)). Since

$$
\Phi_{S^{n}(\pi)}\left(\left[v^{n}\right]\right)=n \Phi_{\pi}([v]) \quad \text { for } 0 \neq v \in \mathcal{H},
$$

it thus follows from Example 5.12 that

$$
I_{S^{n}(\pi)}=n I_{\pi}
$$

For a generalization to more general subrepresentations of $\mathcal{H}^{\otimes n}$, we refer to [8].

\section{2. $C^{*}$-dynamical systems}

Definition 7.4. Let $G$ be a topological group and $\mathcal{A}$ a $C^{*}$-algebra. A $C^{*}$-dynamical system is a triple $(\mathcal{A}, G, \alpha)$, where $\alpha: G \rightarrow \operatorname{Aut}(\mathcal{A}), g \mapsto \alpha_{g}$, is a homomorphism defining a continuous action of $G$ on $\mathcal{A}$.

Theorem 7.5. ([71]) If $G$ is a Banach-Lie group and $(\mathcal{A}, G, \alpha)$ a $C^{*}$-dynamical system, then the space $\mathcal{A}^{\infty}$ of smooth vectors is a Fréchet algebra with respect to the locally convex topology defined by the seminorms

$$
p_{n}(a):=\sup \left\{\left\|\mathrm{d} \alpha\left(x_{1}\right) \cdots \mathrm{d} \alpha\left(x_{n}\right) a\right\|: x_{i} \in \mathfrak{g},\left\|x_{i}\right\| \leq 1\right\}, \quad n \in \mathbb{N}_{0},
$$

and the action of $G$ on $\mathcal{A}^{\infty}$ is smooth. If, in addition, $\mathcal{A}$ is unital, then $\mathcal{A}^{\infty}$ is a continuous inverse algebra.

Now let $(\mathcal{A}, H, \alpha)$ be a $C^{*}$-dynamical system, where $H$ is a Banach-Lie group and $\mathcal{A}$ is a unital $C^{*}$-algebra. Then $\mathrm{U}\left(\mathcal{A}^{\infty}\right)$ carries a natural Fréchet-Lie group structure (cf. Example 3.2(a)), and we can form the semidirect product Lie group $G:=\mathrm{U}\left(\mathcal{A}^{\infty}\right) \rtimes H$.

For the proof of the following theorem, we record a general observation on invariant cones.

Lemma 7.6. If $W \subseteq \mathfrak{g}$ is an open invariant convex cone, then we have for each element $x \in \mathfrak{g}$ satisfying $(\operatorname{ad} x)^{2}=0$ the relation $[x, \mathfrak{g}] \subseteq H(W)$. In particular, $x$ is central if $W$ is pointed. 
Proof. Let $w \in W$. Then, for each $t \in \mathbb{R}$, we have $w+\mathbb{R}[x, w]=e^{\mathbb{R a d} x} w \subseteq W$, so that $[x, w] \in H(W)$ (Lemma 2.9). Now $\mathfrak{g}=W-W$ leads to $[x, \mathfrak{g}] \subseteq H(W)$. If, in addition, $W$ is pointed, this implies that $[x, \mathfrak{g}]=\{0\}$, i.e. $x \in \mathfrak{z}(\mathfrak{g})$.

Theorem 7.7. Let $(\pi, \rho, \mathcal{H})$ be a covariant representation of $(\mathcal{A}, H, \alpha)$, i.e. $\pi$ is a non-degenerate representation of $\mathcal{A}$ on $\mathcal{H}$ and $\rho$ is a unitary representation of $H$ satisfying

$$
\pi\left(\alpha_{g} A\right)=\rho(g) \pi(A) \rho(g)^{-1} \quad \text { for } A \in \mathcal{A}, g \in H
$$

Then the following assertions hold:

(i) The corresponding representation $\widehat{\pi}(a, h):=\pi(a) \rho(h)$ of $G=\mathrm{U}\left(\mathcal{A}^{\infty}\right) \rtimes H$ is smooth if and only if $\rho$ has this property.

(ii) $W_{\widehat{\pi}}=\mathfrak{u}\left(\mathcal{A}^{\infty}\right) \times W_{\rho}$ and $\widehat{\pi}$ is semibounded if and only if $\rho$ has this property if and only if $C_{\widehat{\pi}} \neq \emptyset$.

(iii) If $\mathcal{A}$ is commutative and $\widehat{\pi}$ is semibounded, then the identity component $H_{0}$ of $H$ acts trivially on $\pi(\mathcal{A})$.

Proof. (i) For every $H$-smooth vector $v \in \mathcal{H}$, the smoothness of the map $\mathrm{U}(\mathcal{A}) \times H \rightarrow \mathcal{H},(a, g) \mapsto \pi(a) \rho(g) v$ follows from the smoothness of the action of the Banach-Lie group $\mathrm{U}(\mathcal{A})$ on $\mathcal{H}$. Since the inclusion $\mathrm{U}\left(\mathcal{A}^{\infty}\right) \rightarrow \mathrm{U}(\mathcal{A})$ is smooth, it follows that every $H$-smooth vector is smooth for $G$, so that the corresponding unitary representation $\widehat{\pi}: G \rightarrow \mathrm{U}(\mathcal{H}),(a, g) \mapsto \pi(a) \rho(g)$ of $G$ is smooth whenever $\rho$ has this property.

(ii) Clearly $i \mathbf{1}=\mathrm{d} \pi(i \mathbf{1}) \in \mathrm{d} \widehat{\pi}(\mathfrak{g})$, so that $\widehat{\pi}$ is semibounded if and only if $C_{\widehat{\pi}} \neq \emptyset$ (Proposition 4.7). Since $\mathcal{A}$ acts by bounded operators, we have $\mathfrak{u}\left(\mathcal{A}^{\infty}\right) \subseteq H\left(W_{\widehat{\pi}}\right)$, and thus

$$
W_{\widehat{\pi}}=\mathfrak{u}\left(\mathcal{A}^{\infty}\right) \times\left(W_{\widehat{\pi}} \cap \mathfrak{h}\right)=\mathfrak{u}\left(\mathcal{A}^{\infty}\right) \times W_{\rho} .
$$

Therefore $\widehat{\pi}$ is semibounded if and only if $\rho$ is semibounded.

(iii) As $\mathfrak{u}\left(\mathcal{A}^{\infty}\right)$ is an Abelian ideal of $\mathfrak{g}$, Lemma 7.6 implies that $\left[\mathfrak{u}\left(\mathcal{A}^{\infty}\right), \mathfrak{h}\right] \subseteq$ $H\left(C_{\pi}\right)=\operatorname{ker} \mathrm{d} \widehat{\pi}$, i.e. that $H_{0}$ acts trivially on the $C^{*}$-algebra $\pi(\mathcal{A})$.

Remark 7.8. A closely related fact is well known in the context of $C^{*}$-dynamical systems with the group $H=\mathbb{R}^{d}$. To explain the connection, let $C \subseteq \mathfrak{h}^{\prime}$ be a closed convex cone. Then we say that $(\pi, \rho, \mathcal{H})$ satisfies the $C$-spectrum condition if $-i \mathrm{~d} \rho(x) \geq 0$ for $x \in C^{\star}$, i.e. $I_{\rho} \subseteq C$. If $C^{\star}$ has interior points, we have just seen that the $C$-spectrum condition implies that $\widehat{\pi}$ is semibounded, and if $\pi$ is faithful and $\mathcal{A}$ is commutative, this can only happen if $H$ acts trivially on $\mathcal{A}$ (cf. [10, Theorem IV.6.2]). To obtain nontrivial situations, one has to consider noncommutative algebras. Typical examples arise for any semibounded representation $(\rho, \mathcal{H})$ of $H$ for $\mathcal{A}:=K(\mathcal{H})$ (compact operators on $\mathcal{H}$ ) and $\alpha_{g}(A):=\rho(g) A \rho(g)^{-1}$. 
Example 7.9. (a) If $(\rho, \mathcal{H})$ is a smooth representation of $G$, then the corresponding action of $G$ on $K(\mathcal{H})$ defined by $\alpha_{g}(A):=\rho(g) A \rho(g)^{-1}$ also has a dense space $K(\mathcal{H})^{\infty}$ of smooth vectors because for every pair $(v, w)$ of smooth vectors the corresponding rank-one operator $P_{v, w}$, defined by $P_{v, w}(x):=\langle x, w\rangle v$ satisfies

$$
\alpha_{g} P_{v, w}=P_{\rho(g) v, \rho(g) w},
$$

which easily implies that its orbit map is smooth.

(b) If $\mathcal{H}$ is a complex Hilbert space and $\mathcal{A}:=\operatorname{CAR}(\mathcal{H})$, then the canonical action of the orthogonal group $\mathrm{O}(\mathcal{H})$ (of the underlying real Hilbert space) on $\mathcal{A}$ is continuous and the subalgebra $\mathcal{A}^{\infty}$ of smooth vectors is dense because it contains $a(\mathcal{H})$ and hence the $*$-subalgebra generated by this subset (cf. Sec. 10).

(c) To find a similar situation for the CCR is not so obvious because the Weyl algebra $\operatorname{CCR}(\mathcal{H})$, i.e. the $C^{*}$-algebra defined by the generators $W(f), f \in \mathcal{H}$, and the Weyl relations

$$
W(f)^{*}=W(-f), \quad W(f) W(h)=e^{\frac{i}{2} \operatorname{Im}\langle f, h\rangle} W(f+h)
$$

is a very singular object. The map $W: \mathcal{H} \rightarrow \operatorname{CCR}(\mathcal{H})$ is discontinuous, even on every ray in $\mathcal{H}([14$, Theorem 5.2.8]). Since the action of the symplectic group $\operatorname{Sp}(\mathcal{H})$ preserves these relations, it acts by $\alpha_{g}(W(f))=W(g f)$ on $\operatorname{CCR}(\mathcal{H})$, but this action is highly discontinuous (cf. Sec. 9).

It seems that one possible way out of this dilemma is to find suitable $C^{*}$-algebras consisting of operators with a more regular behavior than $\operatorname{CCR}(\mathcal{H})$. For interesting recent results in this direction we refer to Georgescu's work [23]. Another step in this direction is the construction of a $C^{*}$-algebra $\mathcal{A}$ for each countably dimensional symplectic space whose representations correspond to those representations of the corresponding Weyl relations which are continuous on each one-parameter group $([29])$.

\section{The Virasoro Algebra and Vector Fields on $\mathbb{S}^{1}$}

In this section we discuss invariant cones in the Lie algebra $\mathcal{V}\left(\mathbb{S}^{1}\right)$ of smooth vector fields on the circle and its (up to isomorphy unique) nontrivial central extension $\mathfrak{v i r}$, the Virasoro algebra. For $\mathcal{V}\left(\mathbb{S}^{1}\right)$ we show that, up to sign, there is only one open invariant convex cone given by vector fields of the form $f \frac{\partial}{\partial \theta}$ with $f>0$. As is well known on the Lie algebra level, all unitary highest weight representations of $\mathcal{V}\left(\mathbb{S}^{1}\right)$, resp., the subalgebra of vector fields for which $f$ is a finite Fourier polynomial, are trivial. On the group level we show the closely related result that all semibounded unitary representations of $\operatorname{Diff}\left(\mathbb{S}^{1}\right)_{+}$are trivial. This is the main reason for the Virasoro algebra and the corresponding simply connected group Vir playing a more important role in mathematical physics than Diff $\left(\mathbb{S}^{1}\right)_{+}$itself (cf. $[88,56,77])$. For vir we prove a convexity theorem for adjoint and coadjoint orbits which provides complete information on invariant cones and permits us to determine the momentum sets of the unitary highest weight representations of Vir and to show that they are semibounded. 


\subsection{The invariant cones in $\mathcal{V}\left(\mathbb{S}^{1}\right)$}

We consider $\mathbb{S}^{1}$ as the quotient $\mathbb{R} / 2 \pi \mathbb{Z}$ and identify smooth functions on $\mathbb{S}^{1}$ with the corresponding $2 \pi$-periodic smooth functions on $\mathbb{R}$, where the coordinate is denoted by $\theta$. Accordingly $\partial_{\theta}:=\frac{d}{d \theta}$ is the vector field generating the rigid rotations of $\mathbb{S}^{1}$. We write $G:=\operatorname{Diff}\left(\mathbb{S}^{1}\right)_{+}^{\text {op }}$ for the group of orientation preserving diffeomorphisms endowed with the group structure defined by $\varphi \cdot \psi:=\psi \circ \varphi$, so that $\mathfrak{g}=\mathcal{V}\left(\mathbb{S}^{1}\right)=C^{\infty}\left(\mathbb{S}^{1}\right) \partial_{\theta}$ is its Lie algebra (cf. Examples 3.2(e)). We represent orientation preserving diffeomorphisms of $\mathbb{S}^{1}$ by smooth functions $\varphi: \mathbb{R} \rightarrow \mathbb{R}$ satisfying $\varphi(\theta+2 \pi)=\varphi(\theta)+2 \pi$ for $\theta \in \mathbb{R}$ and $\varphi^{\prime}>0$.

In the following it will be convenient to consider the spaces

$$
\mathcal{F}_{s}\left(\mathbb{S}^{1}\right):=C^{\infty}\left(\mathbb{S}^{1}\right)(d \theta)^{s}, \quad s \in \mathbb{R},
$$

of $s$-densities on $\mathbb{S}^{1}$. Here $(d \theta)^{s}$ denotes the canonical section of the $s$-density bundle of $\mathbb{S}^{1}$ and $G$ acts on $\mathcal{F}_{s}\left(\mathbb{S}^{1}\right)$ by pullbacks

$$
\varphi^{*}\left(u(d \theta)^{s}\right)=(u \circ \varphi)\left(\varphi^{*} d \theta\right)^{s}=(u \circ \varphi)\left(\varphi^{\prime}\right)^{s}(d \theta)^{s} .
$$

The corresponding derived action of the Lie algebra $\mathcal{V}\left(\mathbb{S}^{1}\right)$ is given by the Lie derivative

$$
\mathcal{L}_{f \partial_{\theta}}\left(u(d \theta)^{s}\right)=\left(f u^{\prime}+s f^{\prime} u\right)(d \theta)^{s}
$$

The space $\mathcal{F}_{1}$ is the space of 1 -forms and $\mathcal{F}_{-1} \cong \mathcal{V}\left(\mathbb{S}^{1}\right)$ is the space of vector fields on which (16) describes the adjoint action. We have equivariant multiplication maps $\mathcal{F}_{s} \times \mathcal{F}_{t} \rightarrow \mathcal{F}_{s+t}$, and an invariant integration map

$$
I: \mathcal{F}_{1} \rightarrow \mathbb{R}, \quad f d \theta \mapsto \int_{0}^{2 \pi} f(\theta) d \theta
$$

which leads to an invariant pairing $\mathcal{F}_{-1} \times \mathcal{F}_{2} \rightarrow \mathbb{R}$, and hence to an equivariant embedding

$$
\mathcal{F}_{2} \hookrightarrow \mathcal{V}\left(\mathbb{S}^{1}\right)^{\prime}=\mathfrak{g}^{\prime}
$$

Its image is called the smooth dual of $\mathfrak{g}$. Identifying it with $\mathcal{F}_{2}$, the coadjoint action of $G$ on $\mathfrak{g}^{\prime}$ corresponds to the natural action on $\mathcal{F}_{2}$.

In view of (16), the adjoint action clearly preserves the open cone

$$
W_{\mathcal{V}\left(\mathbb{S}^{1}\right)}:=\left\{f \partial_{\theta}: f>0, f \in C^{\infty}\left(\mathbb{S}^{1}\right)\right\}
$$

of all vector fields corresponding to positive functions. Since every positive function $f$ with $\int_{0}^{2 \pi} f d \theta=2 \pi$ arises as $\varphi^{\prime}$ for some $\varphi \in G$, each $G$-orbit in $W_{\mathcal{V}\left(\mathbb{S}^{1}\right)}$ intersects the (maximal) Abelian subalgebra $\mathfrak{t}:=\mathbb{R} \partial_{\theta}$. We also have a projection map

$$
p_{\mathfrak{t}}: \mathfrak{g} \rightarrow \mathfrak{t}, \quad f \partial_{\theta} \mapsto \frac{1}{2 \pi} \int_{0}^{2 \pi} f(s) d s \cdot \partial_{\theta}=\int_{T} \operatorname{Ad}(\varphi)\left(f \partial_{\theta}\right) d \mu_{T}(\varphi)
$$


where $T:=\exp (\mathfrak{t}) \cong \mathbb{T}$ is the group of rigid rotations and $\mu_{T}$ the normalized Haar measure on $T$.

The following lemma provides some fine information on the convex geometry of adjoint orbits in $W_{\mathcal{V}\left(\mathbb{S}^{1}\right)}$.

Lemma 8.1. The function $\chi: W_{\mathcal{V}\left(\mathbb{S}^{1}\right)} \rightarrow \mathbb{R}, f \partial_{\theta} \mapsto \chi(f):=\frac{1}{2 \pi} \int_{0}^{2 \pi} \frac{1}{f} d \theta$ has the following properties:

(i) It is smooth, G-invariant and strictly convex.

(ii) If the sequence $\left(f_{n}\right)$ in $W_{\mathcal{V}\left(\mathbb{S}^{1}\right)}$ converges to $f \in \partial W_{\mathcal{V}\left(\mathbb{S}^{1}\right)}$, then $\chi\left(f_{n}\right) \rightarrow \infty$.

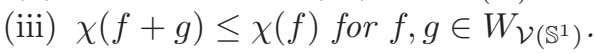

(iv) For each $c>0$, the set $I_{c}:=\left\{x \in W_{\mathcal{V}\left(\mathbb{S}^{1}\right)}: \chi(x) \leq \frac{1}{c}\right\}$ is an invariant closed convex subset of $\mathfrak{g}$ with $\lim \left(I_{c}\right)=\overline{W_{\mathcal{V}\left(\mathbb{S}^{1}\right)}}$. Its boundary is a single orbit

$$
\mathcal{O}_{c \partial_{\theta}}=\partial I_{c}=\operatorname{Ext}\left(I_{c}\right)=\left\{f \in W_{\mathcal{V}\left(\mathbb{S}^{1}\right)}: \chi(f)=\frac{1}{c}\right\}
$$

which coincides with its set of extreme points and satisfies $I_{c}=\overline{\operatorname{conv}}\left(\mathcal{O}_{c \partial_{\theta}}\right)$.

(v) $p_{\mathfrak{t}}\left(\mathcal{O}_{c \partial_{\theta}}\right)=p_{\mathfrak{t}}\left(I_{c}\right)=\left[c, \infty\left[\cdot \partial_{\theta}\right.\right.$ and the only inverse image of the "minimal value" $c \partial_{\theta}$ is the element $c \partial_{\theta}$ itself.

Proof. (i) The $G$-invariance of $\chi$ follows immediately from the Substitution Rule. The function $\chi$ is convex because inversion is a convex function on $\mathbb{R}_{+}^{\times}$and integrals of convex functions are convex. It is smooth because on $W_{\mathcal{V}\left(\mathbb{S}^{1}\right)}$ pointwise inversion is a smooth operation (since $C^{\infty}\left(\mathbb{S}^{1}, \mathbb{R}\right)$ is a real continuous inverse algebra; [24]), and integration is a continuous linear functional.

To verify that $\chi$ is strictly convex, we observe that

$$
\left(\partial_{h} \chi\right)(f)=-\frac{1}{2 \pi} \int_{0}^{2 \pi} \frac{h}{f^{2}} d \theta \text { and }\left(\partial_{h}^{2} \chi\right)(f)=\frac{1}{\pi} \int_{0}^{2 \pi} \frac{h^{2}}{f^{3}} d \theta,
$$

which is positive definite for each $f>0$.

(ii) Now we turn to the boundary behavior of $\chi$. Suppose that the sequence $\left(f_{n}\right)$ in $W_{\mathcal{V}\left(\mathbb{S}^{1}\right)}$ tends to a boundary point $f \in \partial W_{\mathcal{V}\left(\mathbb{S}^{1}\right)}$. Then $f\left(\theta_{0}\right)=0$ for some $\theta_{0} \in\left[0,2 \pi\left[\right.\right.$, and $f \geq 0$ implies that we also have $f^{\prime}\left(\theta_{0}\right)=0$, hence $f(\theta) \leq C\left(\theta-\theta_{0}\right)^{2}$ in a compact $\delta$-neighborhood $U$ of $\theta_{0}$. Given $\varepsilon>0$, we eventually have $f_{n} \leq$ $2 C\left(\theta-\theta_{0}\right)^{2}+\varepsilon$ on $U$ (here we use $C^{2}$-convergence), and therefore

$$
2 \pi \chi\left(f_{n}\right) \geq \int_{\theta_{0}-\delta}^{\theta_{0}+\delta} \frac{1}{f_{n}(\theta)} d \theta \geq \int_{-\delta}^{\delta} \frac{1}{2 C \theta^{2}+\varepsilon} d \theta .
$$

Since $\int_{-\delta}^{\delta} \frac{1}{\theta^{2}} d \theta=\infty$, the Monotone Convergence Theorem implies that

$$
\lim _{\varepsilon \rightarrow 0} \int_{-\delta}^{\delta} \frac{1}{2 C \theta^{2}+\varepsilon} d \theta=\infty
$$

and therefore that $\chi\left(f_{n}\right) \rightarrow \infty$. It also follows that $\int_{0}^{2 \pi} \frac{1}{f(\theta)} d \theta=\infty$.

(iii) If $f, g \in W_{\mathcal{V}\left(\mathbb{S}^{1}\right)}$, then $\frac{1}{f+g} \leq \frac{1}{f}$ implies the assertion. 
(iv) From (ii) we derive that $I_{c}$ is closed in $\mathfrak{g}$. Its invariance follows from the invariance of $\chi$ and its convexity from the convexity of $\chi$. The boundary of $I_{c}$ is a level set of $\chi$, and since every orbit in $W_{\mathcal{V}\left(\mathbb{S}^{1}\right)}$ meets $\mathbb{R}_{+} \partial_{\theta}$ in a unique point and $\left.\chi\right|_{\mathbb{R}_{+}^{\times} \partial_{\theta}}$ is injective, it follows that $\partial I_{c}=\mathcal{O}_{c \partial_{\theta}}$. The fact that $\chi$ is strictly convex further implies that $\partial I_{c} \subseteq \operatorname{Ext}\left(I_{c}\right)$, and since the converse inclusion is trivial, equality follows.

From (iii) we derive that $W_{\mathcal{V}\left(\mathbb{S}^{1}\right)} \subseteq \lim \left(I_{c}\right)$, so that the closedness of $\lim \left(I_{c}\right)$

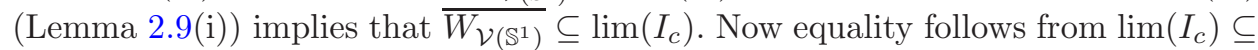
$\lim \left(W_{\mathcal{V}\left(\mathbb{S}^{1}\right)}\right)=\overline{W_{\mathcal{V}\left(\mathbb{S}^{1}\right)}}($ Lemma $2.9(\mathrm{ii}))$

To see that $I_{c}$ coincides with the closed convex hull $D:=\overline{\operatorname{conv}}\left(\mathcal{O}_{c \partial_{\theta}}\right)$, we first observe that we trivially have $D \subseteq I_{c}$. Next we note that $\partial_{\theta}$ is contained in the 3-dimensional subalgebra

$$
\mathfrak{s}:=\operatorname{span}\{1, \cos (\theta), \sin (\theta)\} \partial_{\theta} \cong \mathfrak{s l}_{2}(\mathbb{R})
$$

corresponding to the action of $\mathrm{SL}_{2}(\mathbb{R})$ on $\mathbb{P}_{1}(\mathbb{R}) \cong \mathbb{S}^{1}$. In $\mathfrak{s}$ the element $\partial_{\theta}$ corresponds to the matrix

$$
u=\left(\begin{array}{rr}
0 & 1 \\
-1 & 0
\end{array}\right)
$$

so that Example 6.8 implies that the corresponding group $S:=\langle\exp \mathfrak{s}\rangle \cong \operatorname{PSL}_{2}(\mathbb{R})$ satisfies $p_{\mathfrak{t}}\left(\operatorname{Ad}(S) \partial_{\theta}\right)=\left[1, \infty\left[\cdot \partial_{\theta}\right.\right.$ (cf. Example 6.12) and from that we derive in particular that

$$
\left[c, \infty\left[\cdot \partial_{\theta} \subseteq p_{\mathfrak{t}}\left(\mathcal{O}_{c \partial_{\theta}}\right) \subseteq D,\right.\right.
$$

so that $I_{c}=\operatorname{Ad}(G)\left(\left[c, \infty\left[\cdot \partial_{\theta}\right)\right.\right.$ leads to $I_{c} \subseteq D$.

(v) We have already seen in (18) above that $\left(\partial_{h} \chi\right)(c)=-\frac{1}{2 \pi c^{2}} \int_{0}^{2 \pi} h d \theta$, so that $p_{\mathfrak{t}}^{-1}\left(c \partial_{\theta}\right)$ is a tangent hyperplane of the strictly convex set $I_{c}$. This implies that $c \partial_{\theta}$ is the unique minimum of the linear functional $-\mathrm{d} \chi(c)$ on $I_{c}$ and hence that $p_{\mathrm{t}}^{-1}\left(c \partial_{\theta}\right) \cap I_{c}=\left\{c \partial_{\theta}\right\}$.

We also conclude that $p_{\mathfrak{t}}\left(I_{c}\right) \subseteq\left[c, \infty\left[\cdot \partial_{\theta}\right.\right.$, so that (v) follows from (19).

Remark 8.2. The topological dual $\mathcal{V}\left(\mathbb{S}^{1}\right)^{\prime}$ of $\mathcal{V}\left(\mathbb{S}^{1}\right)=C^{\infty}\left(\mathbb{S}^{1}\right) \partial_{\theta}$ naturally identifies with the space of distributions on $\mathbb{S}^{1}$. Then each $\lambda \in W_{\mathcal{V}\left(\mathbb{S}^{1}\right)}^{\star}$ is a distribution satisfying $\lambda(f) \geq 0$ for $f \geq 0$, and this implies that $\lambda$ extends continuously from $C^{\infty}\left(\mathbb{S}^{1}\right)$ to the Banach space $C\left(\mathbb{S}^{1}\right)$ and thus defines a (finite) positive Radon measure on $\mathbb{S}^{1}$ (cf. [87, Chap. I, Sec. 4, Theorem V]). This shows that the functionals in $W_{\mathcal{V}\left(\mathbb{S}^{1}\right)}^{\star}$ satisfy a strong regularity condition.

Theorem 8.3. (Classification of open invariant cones in $\mathcal{V}\left(\mathbb{S}^{1}\right)$ ) The two open cones $\pm W_{\mathcal{V}\left(\mathbb{S}^{1}\right)}$ are the only non-empty proper open invariant cones in $\mathcal{V}\left(\mathbb{S}^{1}\right)$.

Proof. Let $C \subseteq \mathcal{V}\left(\mathbb{S}^{1}\right)$ be a non-empty open invariant cone. Then $C$ is in particular invariant under the adjoint action of the rotation group $T \cong \mathbb{T}$, generated by the 
vector field $d:=\partial_{\theta}$. Averaging over $T$, we see that either $d$ or $-d$ is contained in $C$ (Proposition 2.11), which leads to $W_{\mathcal{V}\left(\mathbb{S}^{1}\right)} \subseteq C$ or $-W_{\mathcal{V}\left(\mathbb{S}^{1}\right)} \subseteq C$.

Let us assume that $W_{\mathcal{V}\left(\mathbb{S}^{1}\right)} \subseteq C$. Now we apply the same argument to the dual cone $C^{\star} \subseteq W_{\mathcal{V}\left(\mathbb{S}^{1}\right)}^{\star}$ (Proposition 2.11(b)). If $C$ is proper, then $C^{\star}$ contains a nonzero functional $\lambda$, and then $d \in C$ leads to $\lambda(d)>0$. Averaging over $T$ now leads to a $T$-invariant functional $\lambda^{*} \in C^{\star} \subseteq W_{\mathcal{V}\left(\mathbb{S}^{1}\right)}^{\star}$ satisfying $\lambda^{*}(d)=\lambda(d)>0$. We conclude that $\lambda^{*}$ is a positive multiple of the invariant measure $\mu=d \theta$ on $\mathbb{S}^{1} \cong \mathbb{R} / 2 \pi \mathbb{Z}$ (Remark 8.2), which corresponds to the constant function $1 \in \mathcal{F}_{2}$, so that

$$
\operatorname{Ad}^{*}(\varphi) \mu=\left(\varphi^{\prime}\right)^{2} \mu
$$

follows from (16). For any $f \partial_{\theta} \in C$ we now find $\int_{0}^{2 \pi} \psi(\theta)^{2} f(\theta) d \theta \geq 0$ for each positive function $\psi$ with $\int_{0}^{2 \pi} \psi(\theta) d \theta=2 \pi$ (these are precisely the functions occurring as $\varphi^{\prime}$ for an orientation preserving diffeomorphism), and this implies the corresponding relation for all non-negative functions $\psi$ with the integral $2 \pi$. If $f\left(\theta_{0}\right)<0$, then we may choose $\psi$ supported by the set $\{f<0\}$ and obtain a contradiction. This proves that $C \subseteq \overline{W_{\mathcal{V}\left(\mathbb{S}^{1}\right)}}$ and hence that $C=W_{\mathcal{V}\left(\mathbb{S}^{1}\right)}$ because $W_{\mathcal{V}\left(\mathbb{S}^{1}\right)}=\left(\overline{W_{\mathcal{V}\left(\mathbb{S}^{1}\right)}}\right)^{0}$ (Lemma 2.8).

Proposition 8.4. For $\lambda \in \mathcal{V}\left(\mathbb{S}^{1}\right)^{\prime}$ the following are equivalent:

(i) $\mathcal{O}_{\lambda}$ is semi-equicontinuous.

(ii) $\inf \mathcal{O}_{\lambda}\left(\partial_{\theta}\right)>-\infty$ or $\sup \mathcal{O}_{\lambda}\left(\partial_{\theta}\right)<\infty$.

(iii) $\lambda \in W_{\mathcal{V}\left(\mathbb{S}^{1}\right)}^{\star} \cup-W_{\mathcal{V}\left(\mathbb{S}^{1}\right)}^{\star}$.

In particular, $\inf \mathcal{O}_{\lambda}\left(\partial_{\theta}\right)>-\infty$ implies $\mathcal{O}_{\lambda}\left(\partial_{\theta}\right) \geq 0$.

Proof. We have to verify the assumptions of Lemma 6.15 for $d:=\partial_{\theta}$. First, $W_{\mathcal{V}\left(\mathbb{S}^{1}\right)}$ is the open convex cone generated by the orbit $\mathcal{O}_{d}$. Second, the projection $p_{\mathfrak{t}}: \mathfrak{g} \rightarrow \mathbb{R} d=\mathfrak{t}$ is the fixed point projection for the adjoint action of the circle group $T$, hence preserves open and closed convex subsets (Proposition 2.11). Finally, we recall from Lemma $8.1(\mathrm{v})$ that $p_{\mathfrak{t}}\left(\mathcal{O}_{d}\right)=[1, \infty[d$ is unbounded. Now the assertion follows from Lemma 6.15.

Theorem 8.5. The quotient $M:=G / T$ of the group $G=\operatorname{Diff}\left(\mathbb{S}^{1}\right)+$ by the subgroup $T$ of rigid rotations carries the structure of a complex Fréchet manifold on which $G$ acts smoothly by holomorphic maps. Here the tangent space $T_{x_{0}}(G / T)$ in the base point $x_{0}=\mathbf{1 T}$ is canonically identified with $\mathfrak{g}_{\mathbb{C}} / \mathfrak{p}$, where

$$
\mathfrak{p}=\left\{f \partial_{\theta} \in C^{\infty}\left(\mathbb{S}^{1}, \mathbb{C}\right) \partial_{\theta}: f=\sum_{n \leq 0}^{\infty} a_{n} e^{i n \theta}, a_{n} \in \mathbb{C}\right\} .
$$

For each element $x \in W_{\mathcal{V}\left(\mathbb{S}^{1}\right)}$, the flow on $M$ defined by $(t, g T) \mapsto \exp (t x) g T$ extends to a smooth flow on the upper half plane $\mathbb{C}_{+}$which is holomorphic on $\mathbb{C}_{+}^{0} \times M$. 
Proof. The complex structure on $M$ has been discovered by Kirillov and Yuriev (cf. [44, 46, 78]; see in particular [50] for rigorous arguments concerning the complex Fréchet manifold structure). Complex structures always come in pairs, and we therefore write $\bar{M}$ for the same smooth manifold, endowed with the opposite complex structure.

According to [46], the complex manifold structure on $\bar{M}$ can be obtained by identifying it with the space $\mathcal{F}_{\text {reg }}$ of normalized regular univalent functions $f: \mathcal{D} \rightarrow \mathbb{C}$, where $\mathcal{D}$ is the open unit disc in $\mathbb{C}$. These are the injective holomorphic maps $f: \mathcal{D} \rightarrow \mathbb{C}$ extending smoothly to the closure of $\mathcal{D}$ and satisfying $f(0)=0$ and $f^{\prime}(0)=1$ (cf. [30, Sec. 6.5.6] for a detailed discussion). Here the complex structure is determined by its action on $T_{x_{0}}(G / T) \cong T_{\text {id }_{\mathcal{D}}}\left(\mathcal{F}_{\text {reg }}\right) \cong \mathfrak{g} / \mathfrak{t}$ :

$$
I\left[\left(e^{i n \theta}+e^{-i n \theta}\right) \partial_{\theta}\right]=\left[\left(-i e^{i n \theta}+i e^{-i n \theta}\right) \partial_{\theta}\right] \text { for } n>0 .
$$

Since each $x \in W_{\mathcal{V}\left(\mathbb{S}^{1}\right)}$ is conjugate to a multiple of $\partial_{\theta}$, it suffices to assume that $x=\partial_{\theta}$ is the generator of the rigid rotations of $\mathbb{S}^{1}$. In this case the action of the one-parameter group $T=\exp _{G}(\mathbb{R} x)$ on $\mathcal{F}_{\text {reg }}$ is given explicitly by

$$
R_{\alpha} f=R_{\alpha} \circ f \circ R_{-\alpha}, \quad \text { where } R_{\alpha} z=e^{i \alpha} z, \alpha \in \mathbb{R}
$$

(cf. [30, Proposition 6.5.14]). If $\operatorname{Im} \alpha \leq 0$, then $\left|e^{-i \alpha}\right| \leq 1$, so that $R_{\alpha}(\mathcal{D}) \subseteq \mathcal{D}$ implies that $R_{\alpha} f$ can still be defined as above, is continuous on $\mathbb{C}_{-}:=-\mathbb{C}_{+}$and depends holomorphically on $\alpha$ for $\operatorname{Im} \alpha<0$. This implies the holomorphic extension to $\mathbb{C}_{-}$for the complex manifold $\bar{M}$. For the manifold $M$ we therefore obtain an extension to $\mathbb{C}_{+}$.

The holomorphic extension of actions of one-parameter groups on $G / T$ can be carried much further. As shown by Neretin in [73], one even has a "complex semigroup" containing $G$ in its boundary which acts on $G / T$.

Below we shall use the preceding theorem to identify the momentum sets for the unitary highest weight representations of $\mathfrak{v i r} \mathfrak{C}_{\mathbb{C}}$.

Definition 8.6. For the following we recall some algebraic aspects of $\mathcal{V}\left(\mathbb{S}^{1}\right)$. In the complexification $\mathcal{V}\left(\mathbb{S}^{1}\right)_{\mathbb{C}}$, we consider the elements

$$
d_{n}:=i e^{i n \theta} \partial_{\theta}, \quad n \in \mathbb{Z},
$$

satisfying the commutation relations

$$
\left[d_{n}, d_{m}\right]=(n-m) d_{n+m} .
$$

The standard involution on this Lie algebra is given by $\left(f \partial_{\theta}\right)^{*}=-\bar{f} \partial_{\theta}$, so that $x^{*}=-x$ describes the elements of $\mathcal{V}\left(\mathbb{S}^{1}\right)$. Note that $d_{n}^{*}=d_{-n}$ and in particular $d_{0}^{*}=d_{0}$, so that $d_{0}=i \partial_{\theta}$ is a hermitian element (cf. [41, p. 9]).

Theorem 8.7. All semibounded unitary representations of the group Diff $\left(\mathbb{S}^{1}\right)_{+}$are trivial. 
Proof. Let $(\pi, \mathcal{H})$ be a semibounded unitary representation of $G=\operatorname{Diff}\left(\mathbb{S}^{1}\right)_{+}^{\text {op }}$. Then $W_{\pi} \neq \emptyset$, and in view of Theorem 8.3, we may w.l.o.g. assume that $-\partial_{\theta} \in$ $-W_{\mathcal{V}\left(\mathbb{S}^{1}\right)} \subseteq W_{\pi}$, so that the spectrum of the image of $d_{0}:=i \partial_{\theta} \in \mathfrak{g}_{\mathbb{C}}$ under the derived representation is bounded from below. In view of $\exp _{G}\left(2 \pi i d_{0}\right)=\mathbf{1}$, it is contained in $\mathbb{Z}$ and Proposition 4.11 implies the existence of a smooth unit vector $v \in \mathcal{H}^{\infty}$ which is an eigenvector for the minimal eigenvalue $h$ of $d_{0}$.

Now the relation $\left[d_{0}, d_{n}\right]=-n d_{n}$ implies that $d_{n} \cdot v=0$ for each $n>0$. For $n>0$ we then obtain

$$
\begin{aligned}
\left\langle d_{-n} v, d_{-n} v\right\rangle & =\left\langle d_{-n}^{*} d_{-n} v, v\right\rangle=\left\langle d_{n} d_{-n} v, v\right\rangle=\left\langle\left[d_{n}, d_{-n}\right] v, v\right\rangle \\
& =2 n\left\langle d_{0} v, v\right\rangle=2 n h .
\end{aligned}
$$

This implies in particular that $h \geq 0$ and that $h=0$ implies $d_{n} v=0$ for each $n \in \mathbb{Z}$. Now an easy direct calculation leads to

$$
0 \leq \operatorname{det}\left(\begin{array}{cc}
\left\langle d_{-2 n} v, d_{-2 n} v\right\rangle & \left\langle d_{-n}^{2} v, d_{-2 n} v\right\rangle \\
\left\langle d_{-2 n} v, d_{-n}^{2} v\right\rangle & \left\langle d_{-n}^{2} v, d_{-n}^{2} v\right\rangle
\end{array}\right)=4 n^{3} h^{2}(8 h-5 n)
$$

([41, p. 90]; see also [26]). If $h \neq 0$, this expression is negative for sufficiently large $n$, so that we must have $h=0$. This means that $\mathfrak{g} \cdot v=\{0\}$, and hence that $v \in \mathcal{H}^{G}$ is a fixed vector (cf. [66, Remark II.3.7]).

The preceding argument implies that each semibounded unitary representation $(\pi, \mathcal{H})$ of $G$ on a nonzero Hilbert space satisfies $\mathcal{H}^{G} \neq\{0\}$. Applying this to the representation on the invariant subspace $\left(\mathcal{H}^{G}\right)^{\perp}$, which is also semibounded, we find that this space is trivial, and hence that $\mathcal{H}=\mathcal{H}^{G}$, i.e. the representation is trivial.

Remark 8.8. A smooth unitary representation $(\pi, \mathcal{H})$ of $\operatorname{Diff}\left(\mathbb{S}^{1}\right)_{+}$is said to be a positive energy representation if the operator $-i \mathrm{~d} \pi\left(\partial_{\theta}\right)$ has non-negative spectrum.

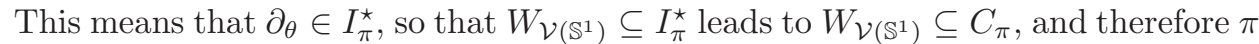
is semibounded. Hence the preceding theorem implies in particular that all positive energy representations of $\operatorname{Diff}\left(\mathbb{S}^{1}\right)+$ are trivial.

Problem 8.9. It would be nice to have an analog of Theorem 8.7 for the universal covering group $\widetilde{G}$ of $G=\operatorname{Diff}\left(\mathbb{S}^{1}\right)_{+}$, which has the fundamental group $\pi_{1}(G)=\mathbb{Z}$. Then $\partial_{\theta}$ generates a subgroup $\widetilde{T} \subseteq \widetilde{G}$ isomorphic to $\mathbb{R}$. Since this group is noncompact, we cannot expect it to have eigenvectors, so that the argument in the proof of Theorem 8.7 does not apply.

What we would need in this context is a suitable direct integral decomposition with respect to the subgroup $Z:=Z(\widetilde{G}) \cong \mathbb{Z}$. If $(\pi, \mathcal{H})$ is a semibounded representation of $\widetilde{G}$ with $\pi(Z) \subseteq \mathbb{T} \mathbf{1}$, i.e. $\pi$ has a central character (which is a consequence of Schur's Lemma if $\pi$ is irreducible), then $\operatorname{Spec}\left(\mathrm{d} \pi\left(d_{0}\right)\right) \subseteq \lambda+\mathbb{Z}$ for some $\lambda \in \mathbb{R}$, and the argument from above applies. This proves that all irreducible semibounded representations of $\widetilde{G}$ are trivial.

We expect that a general semibounded representation of $\widetilde{G}$ has a direct integral decomposition $\mathcal{H} \cong \int_{\widehat{Z}}^{\oplus} \mathcal{H}_{\chi} d \mu(\chi)$ with respect to some measure $\mu$ on $\widehat{Z} \cong \mathbb{T}$ and 
that the semiboundedness of $\pi$ implies that all representations $\pi_{\chi}$ on the spaces $\mathcal{H}_{\chi}$ with central character $\chi$ are semibounded, hence trivial, and this would imply that all semibounded representations of $\widetilde{G}$ are trivial.

\subsection{Invariant cones in the Virasoro algebra}

In the analytic context, the Virasoro algebra is usually defined as the central extension $\mathfrak{v i r}=\mathbb{R} \oplus_{\omega_{\mathrm{GF}}} \mathcal{V}\left(\mathbb{S}^{1}\right)$ defined by the Gelfand-Fuchs cocycle

$$
\omega_{\mathrm{GF}}\left(f \partial_{\theta}, g \partial_{\theta}\right):=\int_{0}^{2 \pi} f^{\prime} g^{\prime \prime} d \theta=\frac{1}{2} \int_{0}^{2 \pi} f^{\prime} g^{\prime \prime}-f^{\prime \prime} g^{\prime} d \theta=\int_{0}^{2 \pi} f^{\prime \prime \prime} g d \theta .
$$

In many situations, the cohomologous cocycle

$$
\begin{aligned}
\omega\left(f \partial_{\theta}, g \partial_{\theta}\right) & :=\int_{0}^{2 \pi}\left(f^{\prime \prime \prime}+f^{\prime}\right) g d \theta=\omega_{\mathrm{GF}}\left(f \partial_{\theta}, g \partial_{\theta}\right)-\frac{1}{2} \int_{0}^{2 \pi} f g^{\prime}-f^{\prime} g d \theta \\
& =\omega_{\mathrm{GF}}\left(f \partial_{\theta}, g \partial_{\theta}\right)-\frac{1}{2} \lambda\left(\left[f \partial_{\theta}, g \partial_{\theta}\right]\right),
\end{aligned}
$$

with $\lambda\left(f \partial_{\theta}\right)=\int_{0}^{2 \pi} f d \theta$, turns out to be more convenient.

Remark 8.10. On the generators $d_{n}=i e^{i n \theta} \partial_{\theta} \in \mathcal{V}\left(\mathbb{S}^{1}\right)_{\mathbb{C}}$ from (20) we have

$$
\omega\left(d_{n}, d_{-n}\right)=2 \pi i\left(n^{3}-n\right) .
$$

With the central element $\hat{c}:=(24 \pi i, 0) \in i \mathfrak{v i r} \subseteq \mathfrak{v i r}_{\mathbb{C}}$, we thus obtain the relation

$$
\left[d_{n}, d_{m}\right]=(n-m) d_{n+m}+\delta_{n,-m} \frac{n^{3}-n}{12} \widehat{c}
$$

if we identify $d_{n}$ with the corresponding element $\left(0, d_{n}\right) \in \mathfrak{v i r}_{\mathbb{C}}([41$, p. 9$])$.

Since we shall need them in the following, we record some related formulas. First we observe that

$$
\mathfrak{t}:=\mathbb{R} c+\mathbb{R} d \quad \text { with } c:=(1,0), d:=\left(0, \partial_{\theta}\right)
$$

is a maximal Abelian subalgebra of vir. The relation $\left[d_{0}, d_{n}\right]=-n d_{n}$ implies that $d_{n} \in \mathfrak{v i r}_{\mathbb{C}}$ is a root vector for the root $\alpha_{n} \in \mathfrak{t}_{\mathbb{C}}^{*}$ defined by

$$
\alpha_{n}(\hat{c})=0 \quad \text { and } \quad \alpha_{n}\left(d_{0}\right)=-n .
$$

In particular,

$$
\alpha_{n}\left(\left[d_{n}, d_{n}^{*}\right]\right)=\alpha_{n}\left(\left[d_{n}, d_{-n}\right]\right)=2 n \alpha_{n}\left(d_{0}\right)=-2 n^{2}<0 \quad \text { for } n \neq 0 .
$$

We also observe that

$$
\begin{aligned}
{\left[d_{n}^{*}, d_{n}\right] } & =\left[d_{-n}, d_{n}\right]=\left(\omega\left(d_{-n}, d_{n}\right),-2 n d_{0}\right)=-i\left(2 \pi\left(n^{3}-n\right), 2 n \partial_{\theta}\right) \\
& =-2 i n\left(\pi\left(n^{2}-1\right), \partial_{\theta}\right) .
\end{aligned}
$$

In view of the $G$-invariant pairing of the space $\mathcal{F}_{2}$ of 2 -densities with vector fields, the 1-cocycle $\mathfrak{g} \rightarrow \mathfrak{g}^{\prime}, x \mapsto i_{x} \omega$ corresponds to the 1-cocycle

$$
\mathfrak{g} \rightarrow \mathcal{F}_{2}, \quad f \partial_{\theta} \mapsto\left(f^{\prime \prime \prime}+f^{\prime}\right)(d \theta)^{2} .
$$


To obtain a formula for the adjoint action of $G=\operatorname{Diff}\left(\mathbb{S}^{1}\right)_{+}^{\text {op }}$ on $\mathfrak{v i r}$, we therefore need a group cocycle $G \rightarrow \mathcal{F}_{2}$ integrating this 1-cocycle (Remark 3.3).

Definition 8.11. The Schwarzian derivative

$$
S(\varphi):=\frac{\varphi^{\prime} \varphi^{\prime \prime \prime}-\frac{3}{2}\left(\varphi^{\prime \prime}\right)^{2}}{\left(\varphi^{\prime}\right)^{2}}=\frac{\varphi^{\prime \prime \prime}}{\varphi^{\prime}}-\frac{3}{2}\left(\frac{\varphi^{\prime \prime}}{\varphi^{\prime}}\right)^{2}
$$

assigns to $\varphi \in G$ a $2 \pi$-periodic smooth function. It satisfies the cocycle identity

$$
S(\varphi \circ \psi)=(S(\varphi) \circ \psi) \cdot\left(\psi^{\prime}\right)^{2}+S(\psi)
$$

(cf. [78]), which means that it defines an $\mathcal{F}_{2}$-valued 1-cocycle on $G$.

We easily derive that $T_{\mathrm{id}}(S)(f)=f^{\prime \prime \prime}$, and therefore the modified Schwarzian derivative

$$
\widetilde{S}(\varphi):=S(\varphi)+\frac{1}{2}\left(\left(\varphi^{\prime}\right)^{2}-1\right)=S(\varphi)+\varphi \cdot \frac{1}{2}-\frac{1}{2}
$$

is a cohomologous 1-cocycle with $T_{\text {id }}(\widetilde{S})(f)=f^{\prime \prime \prime}+f^{\prime}$ (cf. [88, Sec. 7]). Therefore Remark 3.3 implies that the coadjoint action of $G$ on the smooth dual $\mathbb{R} \times \mathcal{F}_{2} \cong$ $\mathbb{R} \times C^{\infty}\left(\mathbb{S}^{1}\right)$ of $\mathfrak{v i r}$ is given by

$$
\operatorname{Ad}_{\varphi}^{*}(a, u)=\left(a,(u \circ \varphi)\left(\varphi^{\prime}\right)^{2}-a \widetilde{S}(\varphi)\right),
$$

whereas, in view of (16), the adjoint action on $\mathfrak{v i r}=\mathbb{R} \oplus_{\omega} \mathcal{V}\left(\mathbb{S}^{1}\right) \cong \mathbb{R} \times C^{\infty}\left(\mathbb{S}^{1}\right)$ is given by

$$
\operatorname{Ad}_{\varphi}(z, f)=\left(z-\int_{0}^{2 \pi} f \widetilde{S}\left(\varphi^{-1}\right) d \theta,(f \circ \varphi) \cdot\left(\varphi^{\prime}\right)^{-1}\right) .
$$

We are especially interested in the adjoint action on the open convex cone

$$
W_{\text {max }}:=\{(z, f) \in \mathfrak{v i r}: f>0\},
$$

which is the inverse image of the positive invariant cone $W_{\mathcal{V}\left(\mathbb{S}^{1}\right)} \subseteq \mathcal{V}\left(\mathbb{S}^{1}\right)$ under the quotient map $\mathfrak{v i r} \rightarrow \mathcal{V}\left(\mathbb{S}^{1}\right),(z, f) \mapsto f$. From the corresponding results for $W_{\mathcal{V}\left(\mathbb{S}^{1}\right)}$, we derive immediately that each orbit in $W_{\max }$ intersects $\mathfrak{t}$.

Proposition 8.12. For each $(z, f) \in W_{\max }$, the $G$-orbit of $(z, f)$ meets $\mathfrak{t}$ in a unique element $(\beta(z, f), \alpha(z, f))$, given by

$$
\alpha(z, f):=\frac{1}{\chi(f)} \quad \text { and } \quad \beta(z, f):=z-\int_{0}^{2 \pi} \frac{\left(f^{\prime}\right)^{2}}{2 f} d \theta+\frac{1}{2} \int_{0}^{2 \pi} f d \theta-\frac{\pi}{\chi(f)} .
$$

For $G=\operatorname{Diff}\left(\mathbb{S}^{1}\right)_{+}$and $T=\exp \left(\mathbb{R} \partial_{\theta}\right)$, the orbit map induces a diffeomorphism

$$
\Gamma: G / T \times\left(W_{\max } \cap \mathfrak{t}\right) \rightarrow W_{\max }, \quad(\varphi T,(\beta, \alpha)) \mapsto \operatorname{Ad}_{\varphi}(\beta, \alpha) .
$$

Proof. Let $(z, f) \in W_{\max }$ and assume that $\operatorname{Ad}_{\varphi^{-1}}(z, f)=(\beta, \alpha) \in \mathfrak{t}$, i.e.

$$
(\beta, \alpha)=\left(z-\int_{0}^{2 \pi} f \widetilde{S}(\varphi) d \theta,\left(f \cdot \varphi^{\prime}\right) \circ \varphi^{-1}\right) .
$$


Then $\varphi^{\prime}=\frac{\alpha}{f}$ leads to $\varphi^{\prime \prime}=-\alpha \frac{f^{\prime}}{f^{2}}$ and

$$
\varphi^{\prime \prime \prime}=-\alpha \frac{f^{\prime \prime}}{f^{2}}+2 \alpha \frac{\left(f^{\prime}\right)^{2}}{f^{3}}=\frac{\alpha}{f^{3}}\left(2\left(f^{\prime}\right)^{2}-f f^{\prime \prime}\right) .
$$

This leads to

$$
\begin{aligned}
S(\varphi) & =\frac{\varphi^{\prime \prime \prime}}{\varphi^{\prime}}-\frac{3}{2}\left(\frac{\varphi^{\prime \prime}}{\varphi^{\prime}}\right)^{2}=\frac{1}{f^{2}}\left(2\left(f^{\prime}\right)^{2}-f f^{\prime \prime}\right)-\frac{3}{2} \frac{\left(f^{\prime}\right)^{2}}{f^{2}}=\frac{1}{2 f^{2}}\left(\left(f^{\prime}\right)^{2}-2 f f^{\prime \prime}\right) \\
& =\frac{\left(f^{\prime}\right)^{2}}{2 f^{2}}-\frac{f^{\prime \prime}}{f}
\end{aligned}
$$

so that we obtain with $\int_{0}^{2 \pi} f^{\prime \prime} d \theta=f^{\prime}(2 \pi)-f^{\prime}(0)=0$ the relation

$$
\int_{0}^{2 \pi} f S(\varphi) d \theta=\int_{0}^{2 \pi} \frac{\left(f^{\prime}\right)^{2}}{2 f} d \theta
$$

Next we use $\varphi^{\prime}=\frac{\alpha}{f}$ to obtain

$$
\frac{1}{2} \int_{0}^{2 \pi} f\left(\left(\varphi^{\prime}\right)^{2}-1\right) d \theta=\frac{1}{2} \int_{0}^{2 \pi} \alpha \varphi^{\prime}-f d \theta=\pi \alpha-\frac{1}{2} \int_{0}^{2 \pi} f d \theta .
$$

Combining all that, we get

$$
\beta=z-\int_{0}^{2 \pi} \frac{\left(f^{\prime}\right)^{2}}{2 f} d \theta+\frac{1}{2} \int_{0}^{2 \pi} f d \theta-\pi \alpha .
$$

We also obtain from $\varphi^{\prime}=\frac{\alpha}{f}$ the relation $\chi(f)=\frac{1}{2 \pi} \int_{0}^{2 \pi} \frac{1}{f} d \theta=\frac{1}{\alpha}$, so that $\alpha=\frac{1}{\chi(f)}$. This proves the first assertion.

Since $T$ fixes the subalgebra $\mathfrak{t}$ pointwise, $\Gamma$ is a well-defined smooth map. As a manifold, we may identify $G / T$ with the set

$$
\left\{\varphi^{\prime}: \varphi \in G\right\}=\left\{h \in C^{\infty}\left(\mathbb{S}^{1}\right): h>0, \int_{0}^{2 \pi} h d \theta=2 \pi\right\} .
$$

As we have seen above, the inverse of $\Gamma$ is given by

$$
\Gamma^{-1}(z, f)=(\varphi,(\beta(z, f), \alpha(z, f))),
$$

where $\varphi^{\prime}=\frac{\alpha}{f}=\frac{1}{f \chi(f)}$, and this map is also smooth. Therefore $\Gamma$ is a diffeomorphism.

The function $\beta$ is rather complicated, but it can be analyzed to some extent as follows. First we observe that for the probability measure $\mu=\frac{1}{2 \pi} d \theta$ Jensen's inequality and the convexity of the function $\frac{1}{x}$ on the positive half line imply that

$$
\frac{1}{\int_{0}^{2 \pi} f d \mu} \leq \int_{0}^{2 \pi} \frac{1}{f} d \mu=\chi(f)=\frac{1}{\alpha},
$$

which implies that $\int_{0}^{2 \pi} f d \theta \geq 2 \pi \alpha$. Therefore the sign of $\beta(0, f)$ is not clear at all, but we shall see below that $\beta(0, f) \leq 0$.

Lemma 8.13. The function $\beta$ is concave. 
Proof. To show that $\beta$ is concave, we have to verify that $\partial_{h}^{2} \beta \leq 0$ in each point of $W_{\max }$. Since $\beta$ is $\operatorname{Ad}(G)$-invariant and each orbit meets $\mathfrak{t}$, it suffices to verify this at points $(z, f)$, where $f$ is constant, so that

$$
\left(\partial_{h}^{2} \beta\right)(z, f)=-f^{-1} \int_{0}^{2 \pi}\left(h^{\prime}\right)^{2} d \theta-\pi \partial_{h}^{2}\left(\chi^{-1}\right)(f) .
$$

Further,

$$
\partial_{h}\left(\chi^{-1}\right)=-\chi^{-2} \partial_{h} \chi \quad \text { and } \quad \partial_{h}^{2}\left(\chi^{-1}\right)=-\chi^{-2}\left(\partial_{h}^{2} \chi\right)+2 \chi^{-3}\left(\partial_{h} \chi\right)^{2},
$$

so that we obtain with $\chi(f)=f^{-1}$ ( $f$ is constant) and the formulas (18) in the proof of Lemma 8.1 the relations

$$
\left(\partial_{h} \chi\right)(f)=-\frac{1}{2 \pi f^{2}} \int_{0}^{2 \pi} h d \theta \quad \text { and } \quad\left(\partial_{h}^{2} \chi\right)(f)=\frac{1}{\pi f^{3}} \int_{0}^{2 \pi} h^{2} d \theta .
$$

This leads further to

$$
\begin{aligned}
\partial_{h}^{2}\left(\chi^{-1}\right)(f) & =-\frac{f^{2}}{\pi f^{3}} \int_{0}^{2 \pi} h^{2} d \theta+2 f^{3} \frac{1}{4 \pi^{2} f^{4}}\left(\int_{0}^{2 \pi} h d \theta\right)^{2} \\
& =\frac{1}{\pi f}\left(-\int_{0}^{2 \pi} h^{2} d \theta+\frac{1}{2 \pi}\left(\int_{0}^{2 \pi} h d \theta\right)^{2}\right) .
\end{aligned}
$$

Putting everything together, we arrive at

$$
f \cdot\left(\partial_{h}^{2} \beta\right)(z, f)=-\int_{0}^{2 \pi}\left(h^{\prime}\right)^{2} d \theta+\int_{0}^{2 \pi} h^{2} d \theta-\frac{1}{2 \pi}\left(\int_{0}^{2 \pi} h d \theta\right)^{2} .
$$

We thus obtain a rotation invariant quadratic form on $C^{\infty}\left(\mathbb{S}^{1}\right)$, so that it is diagonal with respect to Fourier expansion. Evaluating it in the basis functions $\cos (n \theta)$ and $\sin (n \theta)$ immediately shows that it is negative semidefinite.

Theorem 8.14. (Convexity Theorem for adjoint orbits of vir) For each $x \in$ $\mathbb{R} c+\mathbb{R}_{+} \partial_{\theta} \subseteq \mathfrak{t}$, we have

$$
p_{\mathfrak{t}}\left(\mathcal{O}_{x}\right) \subseteq x+C_{+} \quad \text { for } C_{+}:=\mathbb{R}_{+} c+\mathbb{R}_{+} \partial_{\theta} .
$$

If $x$ is not central, then we even have the equality

$$
p_{\mathfrak{t}}\left(\overline{\operatorname{conv}}\left(\mathcal{O}_{x}\right)\right)=x+C_{+} .
$$

Proof. By continuity of the projection $p_{\mathfrak{t}}$, it suffices to assume that $x=\left(\beta_{0}, \alpha_{0}\right)$ with a constant $\alpha_{0}>0$, so that $x \in W_{\max } \cap \mathfrak{t}$. Then $\alpha(x)=\alpha_{0}$ and $\beta(x)=$ $\beta_{0}$. Further, $p_{\mathfrak{t}}\left(\mathcal{O}_{x}\right) \subseteq \overline{\operatorname{conv}}\left(\mathcal{O}_{x}\right)$ (Proposition 2.11), so that the convexity of the functions $\chi=\alpha^{-1}$ and $-\beta$ (Lemma 8.13) implies that for $(\widetilde{\beta}, \widetilde{\alpha}) \in p_{\mathfrak{t}}\left(\mathcal{O}_{x}\right)$, we have

$$
\widetilde{\alpha} \geq \alpha_{0} \text { and } \widetilde{\beta} \geq \beta_{0} .
$$

This means that $p_{\mathfrak{t}}\left(\mathcal{O}_{x}\right) \subseteq x+C_{+}$. 
Now we assume that $x=\beta c+\alpha \partial_{\theta}$ with $\alpha>0$. In view of (25) in Remark 8.10, Proposition C.3 implies that

$$
p_{\mathfrak{t}}\left(\mathcal{O}_{x}\right) \supseteq x+\mathbb{R}^{+} \alpha_{n}(x)\left[d_{n}^{*}, d_{n}\right]=x+\mathbb{R}^{+} \alpha_{n}(-i x) i\left[d_{n}^{*}, d_{n}\right] .
$$

Further $d_{0}=i \partial_{\theta}$ leads to $\alpha_{n}(-i x)>0$, so that

$$
p_{\mathfrak{t}}\left(\mathcal{O}_{x}\right) \supseteq x+\mathbb{R}^{+} i\left[d_{n}^{*}, d_{n}\right], \quad n \in \mathbb{N} .
$$

Next we recall from $(26)$ that

$$
i\left[d_{n}^{*}, d_{n}\right]=2 n\left(\pi\left(n^{2}-1\right), \partial_{\theta}\right)=2 n\left(\pi\left(n^{2}-1\right) c+d\right) .
$$

For $n=1$ we obtain $2\left(0, \partial_{\theta}\right)$, and for $n \rightarrow \infty$ we have positive multiples of $\left(1, \frac{1}{\pi\left(n^{2}-1\right)} \partial_{\theta}\right) \rightarrow c$, so that the closed convex cone generated by the elements $i\left[d_{n}^{*}, d_{n}\right]$ is $C_{+}=\mathbb{R}_{+} c+\mathbb{R}_{+} d$. This proves that

$$
p_{\mathfrak{t}}\left(\overline{\operatorname{conv}}\left(\mathcal{O}_{x}\right)\right)=\overline{\operatorname{conv}}\left(\mathcal{O}_{x}\right) \cap \mathfrak{t} \supseteq x+C_{+},
$$

and our proof is complete.

Note that the following theorem cannot be derived from the "general" Lemma 6.15 because $\mathfrak{t}$ is two-dimensional.

Theorem 8.15. (Classification of open invariant cones in vir) The following statements classify the open invariant convex cones in $\mathfrak{v i r}$ :

(i) Each proper open invariant convex cone in $\mathfrak{v i r}$ is either contained in $W_{\max }$ or $-W_{\max }$.

(ii) Each proper open invariant convex cone $W \subseteq \mathfrak{v i r}$ is uniquely determined by $C:=W \cap \mathfrak{t}$ via $W=\operatorname{Ad}(G) C$.

(iii) Let $C_{\max }:=W_{\max } \cap \mathfrak{t}$. Then an open convex cone $C \subseteq C_{\max }$ is the trace of an invariant open convex cone if and only if $C_{+}^{0} \subseteq C$.

(iv) If $W_{\min }$ is the open invariant cone corresponding to $C_{\min }:=C_{+}^{0}$, then each open invariant convex cone $W \subseteq W_{\max }$ contains $W_{\min }$.

(v) An open invariant convex cone $W$ is pointed if and only if $C$ is pointed. In particular, $W_{\min }$ is pointed.

Proof. (i) Let $W \subseteq \mathfrak{v i r}$ be an open invariant convex cone. If $\mathfrak{z}(\mathfrak{v i r}) \cap W=\emptyset$, then $\mathfrak{z}(\mathfrak{v i r})+W$ is a proper cone, and therefore its image in $\mathcal{V}\left(\mathbb{S}^{1}\right)$ is contained in $W_{\mathcal{V}\left(\mathbb{S}^{1}\right)}$ or $-W_{\mathcal{V}\left(\mathbb{S}^{1}\right)}$, so that $W \subseteq W_{\max }$ or $W \subseteq-W_{\max }$ (Theorem 8.3). To verify (i), we therefore have to show that $c \notin C:=W \cap \mathfrak{t}$. Suppose the converse. Then there exists an $\varepsilon>0$ with $x_{ \pm}:=c \pm \varepsilon \partial_{\theta} \in C$. From Theorem 8.14 we derive that

$$
p_{\mathfrak{t}}\left(\overline{\operatorname{conv}}\left(\mathcal{O}_{x_{+}}\right)\right)=x_{+}+C_{+},
$$

and, applying it also to $-x_{-}$, we find that

$$
p_{\mathfrak{t}}\left(\overline{\operatorname{conv}}\left(\mathcal{O}_{x_{-}}\right)\right)=x_{-}-C_{+} .
$$


Since both sets are contained in $\bar{C}$, we see that $\pm C_{+} \subseteq \lim (\bar{C})=\lim (C)$ (Lemma 2.9), so that $\lim (C)=\mathfrak{t}$, and thus $0 \in C=\mathfrak{t}$. As $W$ is open, $0 \in W$ leads to $W=\mathfrak{v i r}$.

(ii) follows from (i) and Proposition 8.12.

(iii) If $C=W \cap \mathfrak{t}$ for an invariant open convex cone $W$, then $C \subseteq C_{\max }$ implies that $C \cap \mathfrak{z}(\mathfrak{v i r})=\{0\}$. Therefore we have for $x \in C$ the relation

$$
p_{\mathfrak{t}}\left(\overline{\operatorname{conv}}\left(\mathcal{O}_{x}\right)\right)=x+C_{+} \subseteq \bar{C},
$$

and thus $C_{+} \subseteq \lim (\bar{C})=\bar{C}$, which in turn yields $C_{+}^{0} \subseteq C$.

If, conversely, $C_{+}^{0} \subseteq C$ holds for an open convex cone $C \subseteq C_{\max }$, then $C_{+} \subseteq$ $\lim (C)=\bar{C}$ leads for each $x \in C$ to

$$
p_{\mathfrak{t}}\left(\mathcal{O}_{x}\right) \subseteq x+C_{+} \subseteq C .
$$

Therefore

$$
W_{C}:=\left\{x \in \mathfrak{v i r}: p_{\mathfrak{t}}\left(\mathcal{O}_{x}\right) \subseteq C\right\}=\bigcap_{\varphi \in G} \varphi \cdot p_{\mathfrak{t}}^{-1}(C)
$$

is a convex invariant cone containing the subset $\operatorname{Ad}(G) C$ which is open by Proposition 8.12. Hence $W_{C}^{0}$ is an open invariant convex cone satisfying $W_{C}^{0} \cap \mathfrak{t}=C$.

(iv) follows immediately from (iii).

(v) If $W$ is pointed, i.e. it contains no affine lines, then the same holds for $C:=W \cap \mathfrak{t}$. If, conversely, $C$ contains no affine lines, then $H(W)$ is a closed ideal of $\mathfrak{v i r}$ intersecting $\mathfrak{t}$ trivially. Hence it is contained in $[\mathfrak{t}, \mathfrak{v i r}]$ and $H(W)_{\mathbb{C}}$ is adapted to the root decomposition with respect to $\mathfrak{t}_{\mathbb{C}}$. If it contains $d_{n}$, then its *-invariance implies that it also contains $d_{n}^{*}=d_{-n}$, which leads to the contradiction $\left[d_{n}, d_{-n}\right] \in H(W)_{\mathbb{C}}$. This implies that $H(W)=\{0\}$, so that $W$ is pointed.

As a consequence of the preceding theorem, the cones $W_{\min }$, resp., $W_{\max }$ play the role of a minimal, resp., maximal open invariant cone in vir. The existence of minimal and maximal invariant cones is a well-known phenomenon for finite dimensional hermitian Lie algebras (cf. [105] and Proposition 6.16).

Corollary 8.16. The smallest closed convex invariant cone in vir containing $\partial_{\theta}$ is the closure of $W_{\min }$.

Proof. If $D \subseteq \mathfrak{v i r}$ is a closed convex invariant cone, then $\partial_{\theta} \in D$ implies that $\partial_{\theta}+C_{+} \subseteq D$ (Theorem 8.14), so that $C_{+} \subseteq D$ (Lemma 2.9), and thus $W_{\min } \subseteq D$ by invariance.

Remark 8.17. In view of the preceding theorem, the open invariant convex cones in vir can be classified as follows. Since the closure of the cone $C$ contains $C_{+}=$ $\mathbb{R}_{+} c+\mathbb{R}_{+} \partial_{\theta}$ and is contained in $\overline{C_{\max }}=\mathbb{R} c+\mathbb{R}_{+} \partial_{\theta}$, we have $\bar{C}=\mathbb{R}_{+} c+\mathbb{R}_{+}\left(\partial_{\theta}-\alpha c\right)$ for some $\alpha>0$ whenever $C \neq C_{\min }, C_{\max }$. 


\subsection{Semi-equicontinuity of coadjoint orbits of $\mathfrak{v i t}^{\prime}$}

In this final section on the Virasoro algebra we apply the detailed results on invariant cones to semibounded representations and semi-equicontinuous coadjoint orbits. In particular, we show that the set $\mathfrak{g}_{\text {seq }}^{\prime}$ of semi-equicontinuous coadjoint orbits coincides with the double cone $W_{\min }^{\star} \cup-W_{\min }^{\star}$. This in turn is used to show that the unitary highest weight representations of the Virasoro group are precisely the irreducible semibounded representations and to determine their momentum sets.

Proposition 8.18. For $\lambda \in \mathfrak{v i r}^{\prime}$ and $d=\left(0, \partial_{\theta}\right)$, the following are equivalent:

(i) $\mathcal{O}_{\lambda}$ is semi-equicontinuous.

(ii) The convex cone $B\left(\mathcal{O}_{\lambda}\right)$ contains $W_{\max }$ or $-W_{\max }$.

(iii) $\mathcal{O}_{\lambda}(d)=\lambda\left(\mathcal{O}_{d}\right)$ is bounded from below or above.

(iv) $\lambda \in W_{\min }^{\star} \cup-W_{\min }^{\star}$.

(v) $\mathcal{O}_{\lambda}(d) \geq 0$ or $\lambda\left(\mathcal{O}_{d}\right) \leq 0$.

Proof. (i) $\Rightarrow$ (ii): If $\mathcal{O}_{\lambda}$ is semi-equicontinuous, then the invariant convex cone $B\left(\mathcal{O}_{\lambda}\right)$ has interior points and contains $\mathfrak{z}(\mathfrak{v i r})$. Therefore $B\left(\mathcal{O}_{\lambda}\right)^{0} / \mathfrak{z}(\mathfrak{v i r})$ is an open invariant convex cone in $\mathcal{V}\left(\mathbb{S}^{1}\right)$, hence contains $W_{\mathcal{V}\left(\mathbb{S}^{1}\right)}$ or $-W_{\mathcal{V}\left(\mathbb{S}^{1}\right)}$ (Theorem 8.3). This in turn implies that $B\left(\mathcal{O}_{\lambda}\right)$ contains either $W_{\max }$ or $-W_{\max }$.

(ii) $\Rightarrow$ (i) follows from Proposition 2.2 because vir is a Fréchet space.

(ii) $\Rightarrow$ (iii) follows from $d \in W_{\max }$.

(iii) $\Rightarrow$ (ii) follows from $\mathbb{R} c \subseteq B\left(\mathcal{O}_{\lambda}\right)$ and $W_{\max }=\operatorname{Ad}(G)\left(\mathbb{R} c+\mathbb{R}_{+}^{\times} d\right)$ (Proposition 8.12).

(i) $\Rightarrow$ (iv): If $\lambda(c)=0$, then $\mathcal{O}_{\lambda}$ can be identified with a semiequicontinuous coadjoint orbit of $\mathcal{V}\left(\mathbb{S}^{1}\right)$, so that Proposition 8.4 implies that $\lambda \in$ $W_{\mathcal{V}\left(\mathbb{S}^{1}\right)}^{\star} \cup-W_{\mathcal{V}\left(\mathbb{S}^{1}\right)}^{\star}$ in $\mathcal{V}\left(\mathbb{S}^{1}\right)^{\prime}$, which means that $\lambda \in W_{\max }^{\star} \cup-W_{\max }^{\star}$ in $\mathfrak{v i r}^{\prime}$.

If $\lambda(c) \neq 0$, then $\mathcal{O}_{\lambda}$ is contained in the closed invariant hyperplane $\lambda+c^{\perp}$, so that the construction in Remark 2.6 implies that the cone $\mathcal{O}_{\lambda}^{\star}$ has interior points. Clearly, this cone is proper, so that Theorem 8.15 implies that it either contains $W_{\text {min }}$ or $-W_{\text {min }}$, which in turn leads to $\lambda \in W_{\text {min }}^{\star} \cup-W_{\text {min }}^{\star}$.

(iv) $\Rightarrow$ (i): Since $\pm W_{\min }$ are open invariant cones, their duals are semiequicontinuous sets (Example 2.5(b)).

(iv) $\Leftrightarrow(\mathrm{v})$ : Since the closed convex invariant cone generated by $d$ is $W_{\min }$ (Corollary 8.16), $\mathcal{O}_{\lambda}(d) \geq 0$ is equivalent to $\lambda \in W_{\text {min }}^{\star}$.

For any $\lambda \in \mathfrak{t}^{*} \cong[\mathfrak{t}, \mathfrak{g}]^{\perp} \subseteq \mathfrak{g}^{\prime}$, the fact that $\mathcal{O}_{\lambda}$ is constant on the central element $c=(1,0)$ implies that $p_{\mathfrak{t}^{*}}\left(\mathcal{O}_{\lambda}\right) \subseteq \mathfrak{t}^{*}$ is a connected subset of the affine line

$$
\left\{\mu \in \mathfrak{t}^{*}: \mu(c)=\lambda(c)\right\}=\lambda+\left(\mathfrak{t}^{*} \cap c^{\perp}\right) .
$$

In particular, this set is convex. 
Proposition 8.19. If $\lambda \in \mathfrak{t}^{*}$, then

(a) $\mathcal{O}_{\lambda}(d)$ is bounded from below if and only if $\lambda(d) \geq 0$ and $\lambda(c) \geq 0$. If this is the case and $\lambda \neq 0$, then

$$
B\left(\mathcal{O}_{\lambda}\right)^{0}=W_{\max } \quad \text { and } \quad W_{\min } \subseteq \mathcal{O}_{\lambda}^{\star} .
$$

(b) $p_{\mathrm{t}^{*}}\left(\mathcal{O}_{\lambda}\right)$ is contained in an affine half-line if and only if $\mathcal{O}_{\lambda}$ is semiequicontinuous if and only if $\lambda(c) \lambda(d) \geq 0$.

Proof. (a) We recall from Theorem 8.14 that $p_{\mathfrak{t}}\left(\overline{\operatorname{conv}}\left(\mathcal{O}_{d}\right)\right)=d+C_{+}$. This implies that $\lambda\left(\mathcal{O}_{d}\right)$ is bounded from below if and only if $\lambda \in\left(C_{+}\right)^{\star}$, i.e. $\lambda(c), \lambda(d) \geq 0$.

Suppose that these conditions are satisfied and that $\lambda \neq 0$. Then $B\left(\mathcal{O}_{\lambda}\right)^{0}$ is a proper open invariant cone, hence determined by its intersection with $\mathfrak{t}$ (Theorem 8.15). As this intersection contains $d$ and is invariant under translation with $\mathbb{R} c$, Theorem 8.15 implies that it coincides with $C_{\max }$. This proves that $W_{\max }=B\left(\mathcal{O}_{\lambda}\right)^{0}$. We have already seen above that $\lambda \in C_{+}^{\star}$ and since $p_{\mathrm{t}^{*}}\left(\mathcal{O}_{\lambda}\right)$ is a half-line constant on $c$ and bounded below on $d$, it follows that $C_{+} \subseteq \mathcal{O}_{\lambda}^{\star}$, which leads to $W_{\min } \subseteq \mathcal{O}_{\lambda}^{\star}$.

(b) We use Proposition C.3 to obtain with the notation of Remark 8.10

$$
p_{\mathrm{t}^{*}}\left(\mathcal{O}_{\lambda}\right) \supseteq \lambda+\mathbb{R}^{+} \lambda\left(\left[d_{n}^{*}, d_{n}\right]\right) \alpha_{n} .
$$

We also know that $i\left[d_{n}^{*}, d_{n}\right]=2 n\left(\pi\left(n^{2}-1\right), \partial_{\theta}\right)$, so that, for each $n \in \mathbb{N}$,

$$
p_{\mathfrak{t}^{*}}\left(\mathcal{O}_{\lambda}\right) \supseteq \lambda-\mathbb{R}^{+} \lambda\left(\pi\left(n^{2}-1\right), \partial_{\theta}\right) i \alpha_{n}=\lambda-\mathbb{R}^{+} \lambda\left(\pi\left(n^{2}-1\right), \partial_{\theta}\right) i \alpha_{1} .
$$

If $p_{\mathrm{t}^{*}}\left(\mathcal{O}_{\lambda}\right)$ is contained in a half-line, the signs of the numbers

$$
\lambda\left(\pi\left(n^{2}-1\right), \partial_{\theta}\right), \quad n \in \mathbb{N},
$$

have to coincide, which is equivalent to $\lambda(d) \lambda(c) \geq 0$. If, conversely, this condition is satisfied, then (a) implies that $\mathcal{O}_{\lambda}(d)$ is semibounded, so that $p_{\mathrm{t}^{*}}\left(\mathcal{O}_{\lambda}\right)$ is contained in an affine half-line.

Definition 8.20. We write Vir for the (up to isomorphism unique) simply connected Lie group with Lie algeba $\mathfrak{v i r}$.

A unitary representation $(\pi, \mathcal{H})$ of Vir is called a highest weight representation if there exists a smooth cyclic vector $0 \neq v \in \mathcal{H}^{\infty}$ which is a $\mathrm{t}$-eigenvector annihilated by each $d_{n}, n>0$. Then the corresponding eigenfunctional $\lambda \in i t^{*}$ is called the highest weight and $v$ a highest weight vector.

Theorem 8.21. If $\left(\pi_{\lambda}, \mathcal{H}_{\lambda}\right)$ is a unitary highest weight representation of the simply connected Lie group Vir with Lie algebra vir of highest weight $\lambda \in i \mathfrak{t}^{*}$, then

$$
\lambda\left(d_{0}\right) \geq 0 \text { and } \lambda(\widehat{c}) \geq 0,
$$


i.e. $i \lambda\left(\partial_{\theta}\right) \geq 0$ and $i \lambda(c) \geq 0$. The representation $\left(\pi_{\lambda}, \mathcal{H}_{\lambda}\right)$ is semibounded and its momentum set is given by

$$
I_{\pi_{\lambda}}=\overline{\operatorname{conv}}\left(\mathcal{O}_{-i \lambda}\right)
$$

For $\lambda \neq 0$ we have

$$
-W_{\max }=W_{\pi_{\lambda}} \quad \text { and } \quad-W_{\min } \subseteq C_{\pi_{\lambda}} .
$$

Proof. First we use [41, Proposition 3.5] to see that the unitarity of the irreducible highest weight module $L(\lambda)$ of $\mathfrak{v i r}_{\mathbb{C}}$ with highest weight $\lambda \in i \mathfrak{t}^{*}$ implies (28). Actually, this follows from the simple observation that if $v_{\lambda}$ is a highest weight vector of unit length, then

$$
\begin{aligned}
0 & \leq\left\langle d_{-n} v_{\lambda}, d_{-n} v_{\lambda}\right\rangle=\left\langle d_{-n}^{*} d_{-n} v_{\lambda}, v_{\lambda}\right\rangle=\left\langle\left[d_{-n}^{*}, d_{-n}\right] v_{\lambda}, v_{\lambda}\right\rangle \\
& =\lambda\left(\left[d_{n}, d_{-n}\right]\right)=\lambda\left(2 n d_{0}+\frac{n^{3}-n}{12} \widehat{c}\right) \geq 0
\end{aligned}
$$

holds for each $n \in \mathbb{N}$.

The existence of a corresponding continuous unitary highest weight representation $\left(\pi_{\lambda}, \mathcal{H}_{\lambda}\right)$ of the simply connected Lie group Vir with Lie algebra vir has been shown by Goodman and Wallach [28]. A more general method of integration which applies in particular to highest weight modules of Vir has been developed by Toledano Laredo ([101, Theorem 6.1.1]). It is based on techniques related to regular Lie groups, and [101, Corollary 4.2.2] implies in particular that the highest weight vector $v_{\lambda}$ is smooth. This vector is an eigenvector for the closed subalgebra of $\mathfrak{v i r}_{\mathbb{C}}$ generated by $\mathfrak{t}_{\mathbb{C}}$ and $d_{n}, n>0$.

Next we recall from Theorem 8.5 the complex manifold

$$
M=\operatorname{Diff}\left(\mathbb{S}^{1}\right)_{+} / \exp \left(\mathbb{R} \partial_{\theta}\right) \cong \operatorname{Vir} / T,
$$

where $T:=\exp \mathfrak{t} \subseteq$ Vir is the subgroup corresponding to $\mathfrak{t}$. Since the tangent space in the base point $x_{0}=\mathbf{1 T}$ can be identified with $\mathfrak{v i r}_{\mathbb{C}} / \mathfrak{p}, \mathfrak{p}:=\overline{\mathfrak{t}_{\mathbb{C}}+\sum_{n<0} \mathbb{C} d_{n}}$, Theorem 5.11 implies that the map

$$
\eta: M \rightarrow \mathbb{P}\left(\mathcal{H}_{\lambda}^{\prime}\right), \quad g T \mapsto\left[\pi_{\lambda}^{*}(g) \alpha_{v_{\lambda}}\right]
$$

is holomorphic. As $v_{\lambda}$ is cyclic, we thus obtain a realization of the unitary representation $\left(\pi_{\lambda}, \mathcal{H}_{\lambda}\right)$ in the space of holomorphic sections of the holomorphic line bundle $\eta^{*} \mathbb{L}_{\mathcal{H}_{\lambda}^{\prime}}$ over $M$ (cf. Theorem 5.11). ${ }^{\mathrm{a}}$

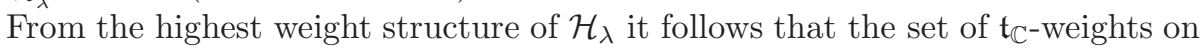
$\mathcal{H}_{\lambda}$ is given by $\lambda-\mathbb{N}_{0} \alpha_{1}$, so that $i \mathrm{~d} \pi_{\lambda}\left(\partial_{\theta}\right)=\mathrm{d} \pi_{\lambda}\left(d_{0}\right)$ is bounded from below. Therefore the cone $C_{\max }$ from the Classification Theorem 8.15 satisfies $-C_{\max } \subseteq W_{\pi_{\lambda}}$, which immediately leads to $-W_{\max } \subseteq W_{\pi_{\lambda}}$. Since $\partial_{\theta} \notin W_{\pi_{\lambda}}$, this cone is proper,

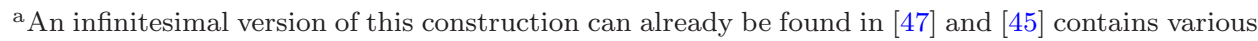
formal aspects of the realization of the highest weight representations in spaces of holomorphic functions, resp., sections of holomorphic line bundles on $M$. 
and the Classification Theorem thus implies the equality $W_{\pi_{\lambda}}=-W_{\max }$. With this information we now apply Theorem 5.7 to determine the precise momentum set.

From Theorem 8.5 it now follows that, for each $x \in W_{\max }=-W_{\pi_{\lambda}}=W_{\pi_{\lambda}^{*}}$, the smooth action of the corresponding one-parameter group on $M$ extends to a holomorphic action of $\mathbb{C}_{+}$. Therefore Theorem 5.11(c) implies that $I_{\pi_{\lambda}}$ is the closed convex hull of the coadjoint orbit $\Phi_{\pi_{\lambda}}\left(G\left[v_{\lambda}\right]\right)$. In view of $\Phi_{\pi_{\lambda}}\left(\left[v_{\lambda}\right]\right)=-i \lambda \in \mathfrak{t}^{*} \subseteq$ $\mathfrak{v i r}^{\prime}$, this proves that $I_{\pi_{\lambda}}=\overline{\operatorname{conv}}\left(\mathcal{O}_{-i \lambda}\right)$. Now

$$
C_{\pi_{\lambda}}=\left(I_{\pi_{\lambda}}^{\star}\right)^{0}=\left(\mathcal{O}_{-i \lambda}^{\star}\right)^{0} \supseteq-W_{\min }
$$

follows directly from Proposition 8.19 , and since $W_{\min }$ has interior points, $\left(\pi_{\lambda}, \mathcal{H}_{\lambda}\right)$ is semibounded.

For more details on the classification of unitary highest weight modules of $\mathfrak{v i r}$, we refer to [41]. We conclude this section with the following converse of Theorem 8.21:

Theorem 8.22. Every irreducible semibounded representation $(\pi, \mathcal{H})$ of Vir is either a highest weight representation or the dual of a highest weight representation.

Proof. We assume that the representation $\pi$ is nontrivial, so that $I_{\pi} \neq\{0\}$. Let $p_{\mathfrak{t}^{*}}: \mathfrak{v i r}^{\prime} \rightarrow \mathfrak{t}^{*}$ be the restriction map. If $p_{\mathfrak{t}^{*}}\left(I_{\pi}\right)=\{0\}$, then $\mathfrak{t} \subseteq I_{\pi}^{\perp}=\operatorname{ker} \mathrm{d} \pi$ and thus $\mathfrak{v i r}=\mathfrak{t}+[\mathfrak{t}, \mathfrak{v i r}] \subseteq$ ker $\mathrm{d} \pi$, contradicting the nontriviality of the representation. Next we use Proposition 2.11 to conclude that $\{0\} \neq p_{\mathrm{t}^{*}}\left(I_{\pi}\right) \subseteq I_{\pi}$. Then each nonzero $\lambda \in \mathfrak{t}^{*} \cap I_{\pi}$ has a semi-equicontinuous orbit, so that $\mathcal{O}_{\lambda}(d)$ is bounded from below or above, and in this case $B\left(\mathcal{O}_{\lambda}\right)^{0}=W_{\max }$ or $-W_{\max }$ (Proposition 8.19). This implies in particular that $W_{\pi} \neq \mathfrak{v i r}$, i.e. $\pi$ is not bounded.

As the open invariant cone $W_{\pi}$ is proper, Theorem 8.15(i) implies that $W_{\pi}$ is either contained in $W_{\max }$ or $-W_{\max }$. We assume the latter and claim that $\pi$ is a highest weight representation. In the other case, $W_{\pi^{*}}=-W_{\pi} \subseteq-W_{\max }$, so that $\pi$ is the dual of a highest weight representation.

First we note that $W_{\pi} \subseteq-W_{\max }$ implies $-W_{\min } \subseteq W_{\pi}$. As $c \in H\left(W_{\pi}\right)$ follows from $\mathrm{d} \pi(c) \in i \mathbb{R} \mathbf{1}$ (Schur's Lemma), we obtain the relation $\mathbb{R} c-W_{\min } \subseteq W_{\pi}$, and therefore $W_{\max }=W_{\min }+\mathbb{R} c$ leads to $W_{\pi}=-W_{\max } \ni-d$. Hence the spectrum of $i \mathrm{~d} \pi(d)=\mathrm{d} \pi\left(d_{0}\right)$ is bounded from below. In view of $\exp (2 \pi d) \in Z$ (Vir), Proposition 4.11 implies the existence of a smooth unit vector $v \in \mathcal{H}^{\infty}$ which is an eigenvector for the minimal eigenvalue $h$ of $i \mathrm{~d} \pi(d)$. Then $\mathfrak{t}=\mathbb{R} c+\mathbb{R} d, v$ is a t-eigenvector and $\left[d_{0}, d_{n}\right]=-n d_{n}$ implies that $d_{n} \cdot v=0$ for each $n>0$. Therefore $(\pi, \mathcal{H})$ is a highest weight representation.

\section{Symplectic Group and Metaplectic Representation}

In this section we study the metaplectic representation $\left(\pi_{s}, S(\mathcal{H})\right)$ of the central extension $\widehat{\operatorname{Sp}}_{\text {res }}(\mathcal{H})$ of $\operatorname{Sp}_{\text {res }}(\mathcal{H})$ on the symmetric Fock space $S(\mathcal{H})$. This representation arises from self-intertwining operators of the Fock representation of the 
Heisenberg group $\operatorname{Heis}(\mathcal{H})$. We show that it is semibounded and determine the corresponding cone $W_{\pi_{s}}$. For the larger central extension $\widehat{\operatorname{HSp}}_{\text {res }}(\mathcal{H})$ of $\mathcal{H} \rtimes \operatorname{Sp}_{\text {res }}(\mathcal{H})$, the representation on $S(\mathcal{H})$ is irreducible and semibounded and we show that its momentum set is the weak-*-closed convex hull of a single coadjoint orbit.

\subsection{The metaplectic representation}

On the dense subspace $S(\mathcal{H})_{0}=\sum_{n=0}^{\infty} S^{n}(\mathcal{H})$ of the symmetric Fock space $S(\mathcal{H})$ (cf. Appendix D) we have for each $f \in \mathcal{H}$ the creation operator

$$
a^{*}(f)\left(f_{1} \vee \cdots \vee f_{n}\right):=f \vee f_{1} \vee \cdots \vee f_{n}
$$

This operator has an adjoint $a(f)$ on $S(\mathcal{H})_{0}$, given by

$$
a(f) \Omega=0, \quad a(f)\left(f_{1} \vee \cdots \vee f_{n}\right)=\sum_{j=0}^{n}\left\langle f_{j}, f\right\rangle f_{1} \vee \cdots \vee \widehat{f}_{j} \vee \cdots \vee f_{n}
$$

where $\widehat{f}_{j}$ means omitting the factor $f_{j}$. Note that $a(f)$ defines a derivation on the algebra $S(\mathcal{H})_{0}$. One easily verifies that these operators satisfy the canonical commutation relations (CCR):

$$
[a(f), a(g)]=0, \quad\left[a(f), a^{*}(g)\right]=\langle g, f\rangle \mathbf{1} .
$$

For each $f \in \mathcal{H}$, the operator $a(f)+a^{*}(f)$ on $S(\mathcal{H})_{0}$ is essentially self-adjoint $([77$, p. 70$])$, so that

$$
W(f):=e^{\frac{i}{\sqrt{2}} \overline{a(f)+a^{*}(f)}} \in \mathrm{U}(\mathcal{H})
$$

is a unitary operator. These operators satisfy the Weyl relations

$$
W(f) W\left(f^{\prime}\right)=W\left(f+f^{\prime}\right) e^{\frac{i}{2} \operatorname{Im}\left\langle f, f^{\prime}\right\rangle}, \quad f, f^{\prime} \in \mathcal{H} .
$$

For the Heisenberg group

$$
\operatorname{Heis}(\mathcal{H}):=\mathbb{R} \times \mathcal{H}
$$

with the multiplication

$$
(t, v)\left(t^{\prime}, v^{\prime}\right):=\left(t+t^{\prime}+\frac{1}{2} \omega\left(v, v^{\prime}\right), v+v^{\prime}\right), \quad \omega\left(v, v^{\prime}\right):=\operatorname{Im}\left\langle v, v^{\prime}\right\rangle
$$

we thus obtain by $W(t, f):=e^{i t} W(f)$ a unitary representation on $S(\mathcal{H})$, called the Fock representation. It is a continuous irreducible representation ([77, Corollary 3.11]). That it actually is smooth follows from the smoothness of

$$
\langle W(t, f) \Omega, \Omega\rangle=e^{i t-\frac{1}{4}\|f\|^{2}}
$$

and Theorem A.3.

Definition 9.1. The symplectic group $\operatorname{Sp}(\mathcal{H})$ acts via $g .(t, v):=(t, g v)$ by automorphism on the Heisenberg group, and since the unitary representation $(W, S(\mathcal{H})$ ) 
is irreducible, there exists for each $g \in \operatorname{Sp}(\mathcal{H})$, up to multiplication with $\mathbb{T}$, at most one unitary operator $\pi_{s}(g) \in \mathrm{U}(S(\mathcal{H}))$ with

$$
\pi_{s}(g) W(t, f) \pi_{s}(g)^{*}=W(t, g f) \quad \text { for } t \in \mathbb{R}, f \in \mathcal{H} .
$$

According to [96], such an operator exists if and only if $g \in \operatorname{Sp}_{\text {res }}(\mathcal{H})$ (cf. Example $3.2(\mathrm{~d})$ ), which immediately leads to a projective unitary representation

$$
\bar{\pi}_{s}: \operatorname{Sp}_{\text {res }}(\mathcal{H}) \rightarrow \mathrm{PU}(S(\mathcal{H})),
$$

determined by $(31)$ for any lift $\pi_{s}(g) \in \mathrm{U}(S(\mathcal{H}))$ of $\bar{\pi}_{s}(g)$. Writing $\bar{u}$ for the image of $u \in \mathrm{U}(\mathcal{H})$ in the projective unitary group $\mathrm{PU}(\mathcal{H})$, the corresponding pull-back

$$
\widehat{\operatorname{Sp}}_{\text {res }}(\mathcal{H}):=\bar{\pi}_{s}^{*} \mathrm{U}(S(\mathcal{H}))=\left\{(g, u) \in \operatorname{Sp}_{\text {res }}(\mathcal{H}) \times \mathrm{U}(S(\mathcal{H})): \bar{u}=\bar{\pi}_{s}(g)\right\}
$$

is called the metaplectic group. It is a central extension of $\operatorname{Sp}_{\text {res }}(\mathcal{H})$ by $\mathbb{T}$. We shall see below that this group is a Lie group and that its canonical representation $\pi_{s}(g, u)=u$ on $S(\mathcal{H})$, the metaplectic representation, is smooth and semibounded. Our strategy is to use Theorem A.4, which requires a suitable lift of $\bar{\pi}_{s}$.

Remark 9.2. From the relation $\mathrm{d} W(f)=\frac{i}{\sqrt{2}}\left(a(f)+a^{*}(f)\right)$, we recover the antilinear and linear part of $\mathrm{d} W$ by

$$
a(f)=\frac{1}{i \sqrt{2}}(\mathrm{~d} W(f)+i \mathrm{~d} W(I f)), \quad a^{*}(f)=\frac{1}{i \sqrt{2}}(\mathrm{~d} W(f)-i \mathrm{~d} W(I f)),
$$

so that by $(31)$

$$
\pi_{s}(g) a(f) \pi_{s}(g)^{-1}=\frac{1}{i \sqrt{2}}(\mathrm{~d} W(g f)+i \mathrm{~d} W(g I f))=a\left(g_{1} f\right)+a^{*}\left(g_{2} f\right)=: a_{g}(f),
$$

where $g=g_{1}+g_{2}$ is the decomposition into linear and antilinear parts.

Theorem 9.3. The topological group $\widehat{\operatorname{Sp}}_{\text {res }}(\mathcal{H})$ is a Lie group and the metaplectic representation is smooth. A Lie algebra cocycle $\eta$ defining $\widehat{\mathfrak{s p}}_{\mathrm{res}}(\mathcal{H})$ as an extension of $\mathfrak{s p}_{\text {res }}(\mathcal{H})$ by $\mathbb{R}$ is given by

$$
\eta(x, y)=\frac{1}{2 i} \operatorname{tr}\left(\left[x_{2}, y_{2}\right]\right),
$$

where $x_{2}$ denotes the antilinear component of $x$.

Proof. Step 1. We consider the unbounded operators $a(f)$ and $a^{*}(f)$ on $S(\mathcal{H})_{0} \subseteq$ $S(\mathcal{H})$ and observe that $\mathbb{C} \Omega$ is the common kernel of the annihilation operators $a(f)$. For $g \in \operatorname{Sp}_{\text {res }}(\mathcal{H})$, we observe that $\pi_{s}(g) \Omega$ lies in the common kernel of the operators $a_{g}(f)=\pi_{s}(g) a(f) \pi_{s}(g)^{-1}$ (cf. Remark 9.2). If $g_{1}$ is invertible, which is the case in some open 1-neighborhood in $\operatorname{Sp}_{\text {res }}(\mathcal{H})$ (actually on the whole group), we consider the antilinear operator $T(g):=g_{2} g_{1}^{-1}$ for which $a_{g}\left(g_{1}^{-1} f\right)=a(f)+a^{*}(T(g) f)$. Therefore $F=\pi_{s}(g) \Omega$ is a solution of the following system of equations:

$$
a(f) F=-a^{*}(T(g) f) F \quad \text { for } f \in \mathcal{H} .
$$


Step 2. For each $n \in \mathbb{N}$, the subset $a^{*}(\mathcal{H}) S^{n}(\mathcal{H})$ is total in $S^{n+1}(\mathcal{H})$, which implies that

$$
\left\{T \in S^{n+1}(\mathcal{H}):(\forall f \in \mathcal{H}) a(f) T=0\right\}=\{0\} .
$$

If an element $F=\sum_{n=0}^{\infty} F_{n} \in S(\mathcal{H})$ with $F_{n} \in S^{n}(\mathcal{H})$ satisfies $(32)$, then

$$
a(f) F_{1}=0, \quad a(f) F_{n+1}=-a^{*}(T(g) f) F_{n-1} \quad \text { for } f \in \mathcal{H}, n \in \mathbb{N} .
$$

This implies $F_{1}=0$, and inductively we obtain with $(33) F_{2 k+1}=0$ for $k \in \mathbb{N}_{0}$. We also derive from (33) that $F$ is completely determined by $F_{0}$, hence that the solution space of (32) is at most one-dimensional.

If $F$ is a solution, we may w.l.o.g. assume that $F_{0}=\Omega$. Then $F_{2}$ satisfies

$$
a(f) F_{2}=-a^{*}(T(g) f) \Omega=-T(g) f, \quad f \in \mathcal{H},
$$

i.e. $F_{2}=-\widehat{T(g)}$ (Lemma D.3). This observation implies Shale's result that only for $g \in \operatorname{Sp}_{\text {res }}(\mathcal{H})$, i.e. $\left\|g_{2}\right\|_{2}<\infty$, Eq. $(31)$ has a solution $\pi_{s}(g) \in \mathrm{U}(S(\mathcal{H}))$.

Step 3. Combining Lemma D.3(i) with Remark D.2, we conclude that the exponential series $e^{-\widehat{T(g)}}$ converges in $S(\mathcal{H})$ for $\|T(g)\|_{2}<1$, which holds on an open 1-neighborhood in $\operatorname{Sp}_{\text {res }}(\mathcal{H})$. Since the operators $a(f)$ act as derivations on $S(\mathcal{H})_{0}$, it follows that

$$
a(f) e^{-\widehat{T(g)}}=-a(f) \widehat{T(g)} \vee e^{-\widehat{T(g)}}=-T(g) f \vee e^{-\widehat{T(g)}}=-a^{*}(T(g) f) e^{-\widehat{T(g)}},
$$

so that $e^{-\widehat{T(g)}}$ satisfies (32). We conclude that

$$
\pi_{s}(g) \Omega=c(g) e^{-\widehat{T(g)}}, \quad c(g) \in \mathbb{C}^{\times} .
$$

Choosing the operators $\pi_{s}(g), g \in \operatorname{Sp}_{\text {res }}(\mathcal{H})$, in such a way that

$$
c(g)=\left\langle\Omega_{g}, \Omega\right\rangle=\left\langle\pi_{s}(g) \Omega, \Omega\right\rangle>0,
$$

it follows that $c(g)=\left\|e^{-\widehat{T(g)}}\right\|^{-1}$ (cf. [77, p. 97]).

Step 4. Since the map $g \mapsto T(g)=g_{2} g_{1}^{-1}$ is smooth in an identity neighborhood and the map

$$
\left\{A \in \mathfrak{p}_{2}:\|A\|_{2}<1\right\} \rightarrow S(\mathcal{H}), \quad A \mapsto e^{\widehat{A}}
$$

is analytic (cf. Remark D.2 and the proof of Lemma $6.6(\mathrm{v})$ for $\mathfrak{p}_{2}$ ), hence in particular smooth, it follows that $e^{-\widehat{T(g)}}$ and hence also $c(g)$ are smooth in an identity neighborhood of $\operatorname{Sp}_{\text {res }}(\mathcal{H})$.

From $\left\langle\pi_{s}(g)^{*} \Omega, \Omega\right\rangle=\left\langle\Omega, \pi_{s}(g) \Omega\right\rangle>0$ we further obtain $\pi_{s}(g)^{*}=\pi_{s}\left(g^{-1}\right)$. This implies that the map

$$
(g, h) \mapsto\left\langle\pi_{s}(g) \pi_{s}(h) \Omega, \Omega\right\rangle=\left\langle\pi_{s}(h) \Omega, \pi_{s}(g)^{*} \Omega\right\rangle=\left\langle\pi_{s}(h) \Omega, \pi_{s}\left(g^{-1}\right) \Omega\right\rangle
$$

is smooth in an identity neighborhood. Now Theorem A.4 implies that $\widehat{\operatorname{Sp}}_{\text {res }}(\mathcal{H})$ is a Lie group and that $\Omega$ is a smooth vector for the corresponding unitary representation, also denoted $\pi_{s}$. Since $\operatorname{Sp}_{\text {res }}(\mathcal{H})$ acts smoothly on $\operatorname{Heis}(\mathcal{H})$, the space $S(\mathcal{H})^{\infty}$ 
of smooth vectors is invariant under $\operatorname{Heis}(\mathcal{H})$, so that the irreducibility of the Fock representation of $\operatorname{Heis}(\mathcal{H})$ on $S(\mathcal{H})([77$, Corollary 3.11]) implies the smoothness of $\pi_{s}$.

Step 5. With Remark A.6 we can now calculate a suitable cocycle $\eta$ with $F(g):=\pi_{s}(g) \Omega=c(g) e^{-\widehat{T(g)}}$ by

$$
\eta(x, y)=2 \operatorname{Im}\langle\mathrm{d} F(\mathbf{1}) x, \mathrm{~d} F(\mathbf{1}) y\rangle+i\langle\mathrm{~d} F(\mathbf{1})[x, y], \Omega\rangle .
$$

As $F(g)$ only depends on $T(g)=g_{2} g_{1}^{-1}$, we have $F(g u)=F(g)$ for $u \in \mathrm{U}(\mathcal{H})$, which leads to $\mathrm{d} F(\mathbf{1}) x=0$ for $x \in \mathfrak{u}(\mathcal{H})$. Hence $\eta(x, \cdot)=0$ for $x \in \mathfrak{u}(\mathcal{H})$. For $x \in \mathfrak{p}_{2}$ we find with $T(\exp x)=\cosh (x) \sinh (x)^{-1}=\tanh (x)$ the relation $\mathrm{d} T(\mathbf{1}) x=x$, and hence

$$
\mathrm{d} F(\mathbf{1}) x=\mathrm{d} c(\mathbf{1})(x) \Omega-\widehat{x} .
$$

Since $c$ is real-valued, this leads with Lemma D.3(ii) for $x, y \in \mathfrak{p}_{2}$ to

$$
\begin{aligned}
\eta(x, y) & =2 \operatorname{Im}\langle\mathrm{d} c(\mathbf{1})(x) \Omega-\widehat{x}, \mathrm{~d} c(\mathbf{1})(y) \Omega-\widehat{y}\rangle=2 \operatorname{Im}\langle\widehat{x}, \widehat{y}\rangle \\
& =\operatorname{Im} \operatorname{tr}(x y)=\frac{1}{2} \operatorname{Im} \operatorname{tr}([x, y])=\frac{1}{2 i} \operatorname{tr}([x, y])
\end{aligned}
$$

because the trace of the symmetric operator $x y+y x$ is real and $\operatorname{tr}([x, y]) \in i \mathbb{R}$.

The content of the preceding theorem is essentially known (cf. [77, 103,96]), although all references known to the author only discuss the metaplectic representation as a representation of a topological group and not as a Lie group. In [77, Theorem 3.19] and [49] one finds quite explicit formulas for group cocycles describing $\widehat{\operatorname{Sp}}_{\text {res }}(\mathcal{H})$ as a central extension.

Remark 9.4. The metaplectic representation of $G:=\widehat{\operatorname{Sp}}_{\text {res }}(\mathcal{H})$ is not irreducible. Since the representation of $\mathrm{U}(\mathcal{H})$ on each $S^{n}(\mathcal{H})$ is irreducible (Example 7.3), every $G$-invariant subspace is the direct sum of some $S^{n}(\mathcal{H}), n \in \mathbb{N}_{0}$.

The preceding proof immediately shows that

$$
\pi(G) \Omega \subseteq S^{\text {even }}(\mathcal{H}):=\widehat{\oplus}_{n \in \mathbb{N}_{0}} S^{2 n}(\mathcal{H})
$$

and that all projections onto the subspaces $S^{2 n}(\mathcal{H})$ are nonzero.

Using the fact that the operators in $\mathrm{d} \pi_{s}(\mathfrak{g})$ contain all multiplication operators with elements $\hat{A}, A \in \mathfrak{p}_{2}$ (cf. Lemma D.3), and their adjoints ([77]), it easily follows that the representations of $G$ on the two subspaces $S^{\text {even }}(\mathcal{H})$ and $S^{\text {odd }}(\mathcal{H})$ are irreducible.

\subsection{The metaplectic group}

In this subsection we describe a convenient description of the metaplectic group $\widehat{\mathrm{Sp}}_{\text {res }}(\mathcal{H})$ as a Banach-Lie group. For further details we refer to [63]. First we recall 
from [63, Definition III.3] that

$$
\begin{aligned}
\operatorname{Sp}_{1,2}(\mathcal{H}):= & \left\{g=\left(\begin{array}{ll}
a & b \\
c & d
\end{array}\right) \in \operatorname{Sp}(\mathcal{H}) \subseteq \mathrm{U}(\mathcal{H}, \mathcal{H}):\right. \\
& \left.\|b\|_{2},\|c\|_{2}<\infty,\|a-\mathbf{1}\|_{1},\|d-\mathbf{1}\|_{1}<\infty\right\}
\end{aligned}
$$

carries the structure of a Banach-Lie group with polar decomposition

$$
\mathrm{U}_{1}(\mathcal{H}) \times \mathfrak{p}_{2} \rightarrow \operatorname{Sp}_{1,2}(\mathcal{H}), \quad \mathfrak{p}_{2}=\left\{\left(\begin{array}{cc}
0 & b \\
b^{*} & 0
\end{array}\right): b=b^{\top} \in B_{2}(\mathcal{H})\right\}
$$

(cf. [63, Definition IV.7, Lemma IV.13]). The full unitary group $\mathrm{U}(\mathcal{H})$ acts smoothly by conjugation on $\operatorname{Sp}_{1,2}(\mathcal{H})$, so that we can form the semidirect product $\operatorname{Sp}_{1,2}(\mathcal{H}) \rtimes \mathrm{U}(\mathcal{H})$, and the multiplication map

$$
\mu_{1}: \operatorname{Sp}_{1,2}(\mathcal{H}) \rtimes \mathrm{U}(\mathcal{H}) \rightarrow \operatorname{Sp}_{\text {res }}(\mathcal{H}), \quad(g, u) \mapsto g u
$$

is a quotient morphism of Banach-Lie groups with kernel

$$
N:=\left\{\left(g, g^{-1}\right): g \in \mathrm{U}_{1}(\mathcal{H})\right\}
$$

so that

$$
\operatorname{Sp}_{\text {res }}(\mathcal{H}) \cong\left(\operatorname{Sp}_{1,2}(\mathcal{H}) \rtimes \mathrm{U}(\mathcal{H})\right) / N
$$

(cf. [63, Definition IV.7]).

From the polar decomposition of $\operatorname{Sp}_{1,2}(\mathcal{H})$ we derive that $\pi_{1}\left(\operatorname{Sp}_{1,2}(\mathcal{H})\right) \cong$ $\pi_{1}\left(\mathrm{U}_{1}(\mathcal{H})\right) \cong \mathbb{Z}$, hence the existence of a unique 2-fold covering group $q: \mathrm{Mp}_{1,2}(\mathcal{H}) \rightarrow \operatorname{Sp}_{1,2}(\mathcal{H})$. On the inverse image $\widehat{\mathrm{U}}_{1}(\mathcal{H})$ of $\mathrm{U}_{1}(\mathcal{H})$ in $\operatorname{Sp}_{1,2}(\mathcal{H})$ we then have a unique character

$$
\sqrt{\text { det }}: \widehat{\mathrm{U}}_{1}(\mathcal{H}) \rightarrow \mathbb{T} \quad \text { with } \mathbf{L}(\sqrt{\text { det }})=\frac{1}{2 i} \operatorname{tr} .
$$

Next we observe that the smooth action of $\mathrm{U}(\mathcal{H})$ on $\operatorname{Sp}_{1,2}(\mathcal{H})$ lifts to a smooth action on $\operatorname{Mp}_{1,2}(\mathcal{H})$. We also note that, for $\operatorname{SU}_{1}(\mathcal{H}):=\operatorname{ker}(\operatorname{det})$, we have $\mathrm{U}_{1}(\mathcal{H}) \cong$ $\mathrm{SU}_{1}(\mathcal{H}) \rtimes \mathbb{T}$, where the determinant is the projection onto the second factor. Accordingly, $\widehat{\mathrm{U}}_{1}(\mathcal{H}) \cong \mathrm{SU}_{1}(\mathcal{H}) \rtimes \mathbb{T}$ with $q(g, t)=\left(g, t^{2}\right)$ and $\sqrt{\operatorname{det}}(g, t)=t$.

Writing $x=x_{1}+x_{2}$ for the decomposition of $x \in \mathfrak{s p}(\mathcal{H})$ into linear and antilinear component, we recall from Theorem 9.3 that

$$
\widehat{\mathfrak{s p}}_{\text {res }}(\mathcal{H}) \cong \mathbb{R} \oplus_{\eta} \mathfrak{s p}_{\text {res }}(\mathcal{H}) \quad \text { with } \eta(x, y)=\frac{1}{2 i} \operatorname{tr}\left(\left[x_{2}, y_{2}\right]\right)
$$

This implies that

$$
\sigma: \mathfrak{s p}_{1,2}(\mathcal{H}) \rightarrow \widehat{\mathfrak{s p}}_{\mathrm{res}}(\mathcal{H}) \cong \mathbb{R} \oplus_{\eta} \mathfrak{s p}_{\mathrm{res}}(\mathcal{H}), \quad \sigma(x):=\left(\frac{1}{2 i} \operatorname{tr}\left(x_{1}\right), x\right)
$$

is a homomorphism of Banach-Lie algebras. Here we use that

$$
[x, y]_{1}=\left[x_{1}, y_{1}\right]+\left[x_{2}, y_{2}\right] \text { and } \operatorname{tr}\left(\left[x_{1}, y_{1}\right]\right)=0 \text {. }
$$


On the subgroup $\widehat{\mathrm{U}}_{1}(\mathcal{H}) \subseteq \mathrm{Mp}_{1,2}(\mathcal{H}), \sigma$ integrates to the group homomorphism

$$
\sigma_{G}: \widehat{\mathrm{U}}_{1}(\mathcal{H}) \rightarrow \widehat{\operatorname{Sp}}_{\mathrm{res}}(\mathcal{H}) \subseteq \operatorname{Sp}_{\text {res }}(\mathcal{H}) \times \mathrm{U}(S(\mathcal{H})), \quad \sigma(g):=\left(q(g), \sqrt{\operatorname{det}}(g) \pi_{s}(g)\right)
$$

so that the polar decomposition of $\operatorname{Sp}_{1,2}(\mathcal{H})$ implies that $\sigma$ integrates to a morphism of Banach-Lie groups $\sigma_{G}: \operatorname{Mp}_{1,2}(\mathcal{H}) \rightarrow \widehat{\operatorname{Sp}}_{\text {res }}(\mathcal{H})$. Combining this map with the canonical inclusion $\mathrm{U}(\mathcal{H}) \hookrightarrow \widehat{\operatorname{Sp}}_{\text {res }}(\mathcal{H})$, the equivariance of $\sigma_{G}$ under conjugation with unitary operators implies the existence of a homomorphism

$$
\mu: \operatorname{Mp}_{1,2}(\mathcal{H}) \rtimes \mathrm{U}(\mathcal{H}) \rightarrow \widehat{\operatorname{Sp}}_{\text {res }}(\mathcal{H}), \quad(g, u) \mapsto \sigma_{G}(g) u
$$

Proposition 9.5. The homomorphism $\mu$ factors through an isomorphism

$$
\bar{\mu}:\left(\operatorname{Mp}_{1,2}(\mathcal{H}) \rtimes \mathrm{U}(\mathcal{H})\right) / \operatorname{ker} \mu \rightarrow \widehat{\operatorname{Sp}}_{\text {res }}(\mathcal{H})
$$

of connected Banach-Lie groups with $\operatorname{ker} \mu \cong \mathrm{SU}_{1}(\mathcal{H})$.

Proof. If $\mu(g, u)=\mathbf{1}$, then $\sigma_{G}(g)=u^{-1}$ implies that $g \in \widehat{\mathrm{U}}_{1}(\mathcal{H})$ with $q(g)=u^{-1}$. Now

$$
\Omega=\pi_{s}(\mu(g, u)) \Omega=\sqrt{\operatorname{det}}(g) \Omega
$$

implies $\sqrt{\operatorname{det}}(g)=1$. This shows that $\operatorname{ker} \mu=\left\{\left(g, q(g)^{-1}\right): g \in \mathrm{SU}_{1}(\mathcal{H})\right\}$, and the assertion follows.

The inverse image of the center $Z \cong \mathbb{T}$ of $\widehat{\operatorname{Sp}}_{\text {res }}(\mathcal{H})$ is

$$
\widehat{N}:=\left\{\left(g, q(g)^{-1}\right): g \in \widehat{\mathrm{U}}_{1}(\mathcal{H})\right\} .
$$

The inverse image $\widehat{\mathrm{U}}(\mathcal{H})$ of the subgroup $\mathrm{U}(\mathcal{H})$ in $\widehat{\mathrm{Sp}}_{\text {res }}(\mathcal{H})$ is of the form

$$
\widehat{\mathrm{U}}(\mathcal{H}) \cong\left(\widehat{\mathrm{U}}_{1}(\mathcal{H}) \rtimes \mathrm{U}(\mathcal{H})\right) / \operatorname{ker} \mu \cong Z \times \mathrm{U}(\mathcal{H}) .
$$

In particular, it splits as a central extension of Lie groups.

\subsection{Semiboundedness of the metaplectic representation}

In this subsection we show that the metaplectic representation $\left(\pi_{s}, S(\mathcal{H})\right)$ of $\widehat{\operatorname{Sp}}_{\text {res }}(\mathcal{H})$ is semibounded and determine the open invariant cone $W_{\pi_{s}}$ as the inverse image of the canonical cone $W_{\mathfrak{s p}_{\text {res }}}(\mathcal{H})$ in $\mathfrak{s p}_{\text {res }}(\mathcal{H})$.

We start with a closer look at adjoint orbits of the symplectic Lie algebra.

Lemma 9.6. If $(V, \omega)$ is a finite dimensional symplectic space and $X \in \mathfrak{s p}(V, \omega)$ is such that the Hamiltonian function $H_{X}(v)=\frac{1}{2} \omega(X v, v)$ is indefinite, then $\operatorname{conv}\left(\mathcal{O}_{X}\right)=\mathfrak{s p}(V, \omega)$.

Proof. Let $\mathfrak{g}:=\mathfrak{s p}(V, \omega)$. Then Proposition 6.16, combined with the uniqueness of the open invariant cone in $\mathfrak{s p}(V, \omega)$ up to sign (cf. [105]), implies that the set of semiequicontinuous coadjoint orbits coincides with the double cone $W_{\mathfrak{s p}(V, \omega)}^{\star} \cup-W_{\mathfrak{s p}(V, \omega)}^{\star}$. 
Using the Cartan-Killing form to identify $\mathfrak{g}$ with its dual, we accordingly see that any semi-equicontinuous adjoint orbit is contained in the double cone

$$
\overline{W_{\mathfrak{s p}(V, \omega)}} \cup-\overline{W_{\mathfrak{s p}(V, \omega)}},
$$

i.e. the corresponding Hamiltonian function is either positive or negative.

Finally, we observe that if $x \in \mathfrak{g}$ satisfies $\operatorname{conv}\left(\mathcal{O}_{x}\right) \neq \mathfrak{g}$, then $B\left(\mathcal{O}_{x}\right)$ is a nonzero invariant cone and Proposition 6.16(i) implies that it has interior points. Therefore $\mathcal{O}_{x}$ is semi-equicontinuous because every finite dimensional space is barreled.

Lemma 9.7. The subspace $\mathfrak{p}:=\{X \in \mathfrak{s p}(\mathcal{H}): I X=-X I\}$ contains no nontrivial Lie algebra ideals, and the same holds for the subspace

$$
\mathfrak{p}_{2}:=\left\{X \in \mathfrak{s p}_{\text {res }}(\mathcal{H}): I X=-X I\right\}=\mathfrak{p} \cap \mathfrak{s p}_{\text {res }}(\mathcal{H})
$$

Proof. First we show that $X \in \mathfrak{p}$ and $[X,[I, X]]=0$ implies $X=0$. Realizing $\mathfrak{s p}(\mathcal{H})$ as a closed subalgebra of $\mathfrak{g l}\left(\mathcal{H}_{\mathbb{C}}\right) \cong \mathfrak{g l}(\mathcal{H} \oplus \mathcal{H})(\mathrm{cf}$. Example 3.2(h)), we have

$$
\mathfrak{p}=\left\{\left(\begin{array}{cc}
0 & a \\
a^{*} & 0
\end{array}\right): a^{\top}=a \in B(\mathcal{H})\right\} \quad \text { and } \quad I=\left(\begin{array}{rr}
i & 0 \\
0 & -i
\end{array}\right)
$$

For $X=\left(\begin{array}{cc}0 & a \\ a^{*} & 0\end{array}\right)$ this leads to

$$
[X,[I, X]]=\left[\left(\begin{array}{cc}
0 & a \\
a^{*} & 0
\end{array}\right),\left(\begin{array}{cc}
0 & 2 i a \\
-2 i a^{*} & 0
\end{array}\right)\right]=-4 i\left(\begin{array}{cc}
a a^{*} & 0 \\
0 & a^{*} a
\end{array}\right) .
$$

If this operator vanishes, then $a^{*} a=0$ implies that $a=0$, so that $X=0$.

If $\mathfrak{i} \subseteq \mathfrak{p}$ is a Lie algebra ideal, then we have for each $X \in \mathfrak{i}$ the relation $[X,[I, X]] \in \mathfrak{i} \cap \mathfrak{u}(\mathcal{H})=\{0\}$, so that the preceding argument shows that $X=0$. The same argument applies to $\mathfrak{p}_{2}=\mathfrak{p} \cap \mathfrak{s p}_{\text {res }}(\mathcal{H})$.

Lemma 9.8. Let $X \in \mathfrak{s p}(\mathcal{H})$. Then the projection $p_{\mathfrak{k}}: \mathfrak{s p}(\mathcal{H}) \rightarrow \mathfrak{u}(\mathcal{H})$ onto the $\mathbb{C}$-linear component satisfies

$$
p_{\mathfrak{k}}\left(\mathcal{O}_{X}\right) \subseteq\{y \in \mathfrak{u}(\mathcal{H}): i y \leq 0\}=\overline{C_{\mathfrak{u}(\mathcal{H})}},
$$

if and only if $H_{X} \geq 0$. A corresponding statement holds for $\mathfrak{s p}_{\mathrm{res}}(\mathcal{H})$.

Proof. Since $p_{\mathfrak{k}}$ is the fixed point projection for the action of the torus $e^{\mathbb{R a d} I}$, it preserves the closed convex cones $\pm\left\{Z \in \mathfrak{s p}(\mathcal{H}): H_{Z} \geq 0\right\}$ (Proposition 2.11), so that the relation $H_{X} \geq 0$ implies $i p_{\mathfrak{k}}\left(\mathcal{O}_{X}\right) \leq 0$ (Theorem 6.7(i)). Suppose, conversely, that $i p_{\mathfrak{k}}\left(\mathcal{O}_{X}\right) \leq 0$. If $H_{X} \leq 0$, then the preceding argument shows that $i p_{\mathfrak{k}}\left(\mathcal{O}_{X}\right) \geq 0$, which leads to $p_{\mathfrak{k}}\left(\mathcal{O}_{X}\right)=\{0\}$, so that the closed span of $\mathcal{O}_{X}$ is an ideal of $\mathfrak{g}$ contained in $\mathfrak{p}=\operatorname{ker} p_{\mathfrak{k}}$. In view of Lemma 9.7, this leads to $X=0$.

We may therefore assume that $H_{X}$ is indefinite. Hence there exists a finite dimensional complex subspace $\mathcal{H}_{1} \subseteq \mathcal{H}$ on which $H_{X}$ is indefinite. Then we have a 
Hilbert space direct sum $\mathcal{H}=\mathcal{H}_{1} \oplus \mathcal{H}_{1}^{\perp}$ and accordingly we write operators on $\mathcal{H}$ as $(2 \times 2)$-block matrices:

$$
X=\left(\begin{array}{ll}
X_{11} & X_{12} \\
X_{21} & X_{22}
\end{array}\right)
$$

For $v \in \mathcal{H}_{1}$ we then have $\operatorname{Im}\langle X v, v\rangle=\operatorname{Im}\left\langle X_{11} v, v\right\rangle$, so that $X_{11} \in \mathfrak{s p}\left(\mathcal{H}_{1}\right)$ and $H_{X_{11}}$ is indefinite.

Identifying $\operatorname{Sp}\left(\mathcal{H}_{1}\right)$ in the natural way with a $\operatorname{subgroup}$ of $\operatorname{Sp}(\mathcal{H})$, Lemma 9.6 implies that $\operatorname{conv}\left(\operatorname{Ad}\left(\operatorname{Sp}\left(\mathcal{H}_{1}\right) X\right)\right)$ contains an element $Y$ with $Y_{11}=-i \mathbf{1}$. From

$$
p_{\mathfrak{k}}(Y)=\left(\begin{array}{ll}
p_{\mathfrak{k}}\left(Y_{11}\right) & p_{\mathfrak{k}}\left(Y_{12}\right) \\
p_{\mathfrak{k}}\left(Y_{21}\right) & p_{\mathfrak{k}}\left(Y_{22}\right)
\end{array}\right)=\left(\begin{array}{cc}
Y_{11} & p_{\mathfrak{k}}\left(Y_{12}\right) \\
p_{\mathfrak{k}}\left(Y_{21}\right) & p_{\mathfrak{k}}\left(Y_{22}\right)
\end{array}\right)
$$

we now derive that $i p_{\mathfrak{k}}(Y) \not \leq 0$, contradicting our assumption on $X$. This completes the proof of the first assertion.

Since $\operatorname{Sp}\left(\mathcal{H}_{1}\right) \subseteq \operatorname{Sp}_{\text {res }}(\mathcal{H})$, the preceding argument also implies the assertion for the restricted Lie algebra $\mathfrak{s p}_{\text {res }}(\mathcal{H})$.

Now we are ready to show that the metaplectic representation is semibounded. We write

$$
q: \widehat{\mathfrak{s p}}_{\text {res }}(\mathcal{H}) \rightarrow \mathfrak{s p}_{\text {res }}(\mathcal{H})
$$

for the quotient map and $\mathfrak{z}$ for its kernel. Since the central extension is trivial over the subalgebra $\mathfrak{u}(\mathcal{H})$ of $\mathfrak{s p}_{\text {res }}(\mathcal{H})$, we have

$$
\widehat{\mathfrak{u}}(\mathcal{H}):=q^{-1}(\mathfrak{u}(\mathcal{H})) \cong \mathfrak{z} \oplus \mathfrak{u}(\mathcal{H}),
$$

where the $\mathfrak{u}(\mathcal{H})$-complement is uniquely determined by the property that it is the commutator algebra. Here we use that the Lie algebra $\mathfrak{u}(\mathcal{H})$ is perfect (cf. [63, Lemma I.3]). Accordingly, we may identify $\mathfrak{u}(\mathcal{H})$ in a natural way with a subalgebra of $\widehat{\mathfrak{s p}}_{\text {res }}(\mathcal{H})$.

Remark 9.9. The momentum set $I_{\pi_{s}}$ is completely determined by the restriction of its support function $s_{\pi_{s}}$ to $W_{\pi_{s}}=B\left(I_{\pi_{s}}\right)^{0}$ (Remark 2.3(b)) which is invariant under the adjoint action. We shall see below that $q\left(W_{\pi_{s}}\right) \subseteq W_{\mathfrak{s p}_{\text {res }}(\mathcal{H})}$, so that Theorem 6.7(ii) leads to

$$
W_{\pi_{s}} \subseteq \operatorname{Ad}\left(\widehat{\operatorname{Sp}}_{\text {res }}(\mathcal{H})\right)\left(\mathfrak{z} \times C_{\mathfrak{u}(\mathcal{H})}\right),
$$

where $\mathfrak{z} \cong \mathbb{R}$ denotes the center. This in turn entails that $I_{\pi_{s}}$ is already determined by the restriction of $s_{\pi_{s}}$ to $\mathfrak{z} \times C_{\mathfrak{u}(\mathcal{H})}$, which we determine below.

This restriction is the support function for the momentum set of the restriction of $\pi_{s}$ to the subgroup $\widehat{\mathrm{U}}(\mathcal{H}) \cong \mathbb{T} \times \mathrm{U}(\mathcal{H})$ (Proposition 4.7(iii)), which is a direct sum of the representations on the subspaces $S^{n}(\mathcal{H})$, on which we have

$$
\pi_{s}(z, g)\left(v_{1} \vee \cdots \vee v_{n}\right)=z g v_{1} \vee \cdots \vee g v_{n} \quad \text { for } v_{1}, \ldots, v_{n} \in \mathcal{H}
$$


For the representation of $\mathrm{U}(\mathcal{H})$ on $S^{n}(\mathcal{H})$, the momentum set $I_{S^{n}(\mathcal{H})}^{\mathrm{U}(\mathcal{H})}$ is simply given by

$$
I_{S^{n}(\mathcal{H})}^{\mathrm{U}(\mathcal{H})}=n I_{\mathcal{H}}^{\mathrm{U}(\mathcal{H})}
$$

(Example 7.3(b)), so that

$$
I_{\pi_{s}}^{\widehat{U}(\mathcal{H})}=\overline{\operatorname{conv}}\left(\bigcup_{n \in \mathbb{N}_{0}}\{1\} \times n I_{\mathcal{H}}^{\mathrm{U}(\mathcal{H})}\right),
$$

which is a convex cone with vertex $(1,0)$, and for $x \in \mathfrak{u}(\mathcal{H})$, we have

$$
s_{\pi_{s}}(t, x)= \begin{cases}-t & \text { for } i x \leq 0 \\ \infty & \text { else. }\end{cases}
$$

The representation of $\widehat{\operatorname{Sp}}_{\text {res }}(\mathcal{H})$ on $S(\mathcal{H})$ decomposes into two irreducible pieces

$$
S^{\text {even }}(\mathcal{H})=\widehat{\oplus}_{n \in \mathbb{N}_{0}} S^{2 n}(\mathcal{H}) \quad \text { and } \quad S^{\text {odd }}(\mathcal{H})=\widehat{\oplus}_{n \in \mathbb{N}_{0}} S^{2 n+1}(\mathcal{H})
$$

(Remark 9.4). Writing $\pi_{ \pm}$for the corresponding representations, we obtain

$$
I_{\pi_{+}}^{\widehat{U}(\mathcal{H})}=I_{\pi_{s}}^{\widehat{U}(\mathcal{H})}=\overline{\mathrm{conv}}\left(\bigcup_{n \in \mathbb{N}_{0}}\{1\} \times 2 n I_{\mathcal{H}}^{\mathrm{U}(\mathcal{H})}\right)
$$

and

$$
I_{\pi_{-}}^{\widehat{U}(\mathcal{H})}=\overline{\operatorname{conv}}\left(\bigcup_{n \in \mathbb{N}_{0}}\{1\} \times(2 n+1) I_{\mathcal{H}}^{\mathrm{U}(\mathcal{H})}\right) .
$$

Now it is easy to derive that $W_{\pi_{ \pm}}=W_{\pi_{s}}, s_{\pi_{+}}=s_{\pi_{s}}$, and that

$$
s_{\pi_{-}}(t, x)=-t+\sup (\operatorname{Spec}(i x)) \quad \text { for } i x \leq 0 .
$$

Theorem 9.10. The metaplectic representation $\left(\pi_{s}, S(\mathcal{H})\right)$ of $\left.\widehat{\operatorname{Sp}}_{\text {res }}(\mathcal{H})\right)$ is semibounded with

$$
W_{\pi_{s}}=W_{\widehat{\mathfrak{p}}_{\mathrm{res}}(\mathcal{H})}:=q^{-1}\left(W_{\mathfrak{s p}_{\mathrm{res}}(\mathcal{H})}\right)
$$

Proof. From Example 4.10 we know that

$$
s_{\pi_{s}}(x) \leq 0 \quad \text { for } x \in C_{\mathfrak{u}(\mathcal{H})}, \quad \text { so that } s_{\pi_{s}}(t, x)=-t+s_{\pi_{s}}(x)<\infty
$$

for $(t, x) \in \mathfrak{z} \times C_{\mathfrak{u}(\mathcal{H})}$. From the invariance of $s_{\pi_{s}}$ under the adjoint action and Theorem 6.7(ii) it now follows that

$$
\operatorname{Ad}\left(\widehat{\operatorname{Sp}}_{\text {res }}(\mathcal{H})\right)\left(\mathfrak{z} \times C_{\mathfrak{u}(\mathcal{H})}\right)=q^{-1}\left(\operatorname{Ad}\left(\operatorname{Sp}_{\text {res }}(\mathcal{H})\right) C_{\mathfrak{u}(\mathcal{H})}\right)=q^{-1}\left(W_{\mathfrak{s p}_{\text {res }}(\mathcal{H})}\right) \subseteq B\left(I_{\pi_{s}}\right)
$$

(cf. Theorem 6.7(ii)). In particular, $B\left(I_{\pi_{s}}\right)$ has interior points, so that $\pi_{s}$ is semibounded with $W_{\widehat{\mathfrak{p}}_{\text {res }}(\mathcal{H})} \subseteq W_{\pi_{s}}$.

To prove equality, we note that (38) in Remark 9.9 implies that

$$
W_{\pi_{s}} \cap \mathfrak{u}(\mathcal{H}) \subseteq\left\{x \in \mathfrak{u}(\mathcal{H}): s_{\pi_{s}}(x)<\infty\right\}=\{x \in \mathfrak{u}(\mathcal{H}): i x \leq 0\}=\overline{C_{\mathfrak{u}(\mathcal{H})}} .
$$


As $W_{\pi_{s}}$ is invariant under the projection $p_{\mathfrak{k}}: \widehat{\mathfrak{s p}}_{\mathrm{res}}(\mathcal{H}) \rightarrow \widehat{\mathfrak{u}}(\mathcal{H})$ onto the fixed point space of the compact group $e^{\mathbb{R a d} I}$ (Proposition 2.11), we obtain for each $x \in W_{\pi_{s}}$ the relation $i p_{\mathfrak{k}}\left(\mathcal{O}_{q(x)}\right) \leq 0$, so that Lemma 9.8 leads to $q(x) \in \overline{W_{\mathfrak{s p}_{\text {res }}}(\mathcal{H})}$. As $W_{\pi_{s}}$ is open and $W_{\mathfrak{s p} \text { res }}(\mathcal{H})$ coincides with the interior of its closure (Lemma 2.8), it follows that

$$
q\left(W_{\pi_{s}}\right) \subseteq W_{\mathfrak{s p} \text { res }}(\mathcal{H})
$$

Combining this with (39), we obtain $q\left(W_{\pi_{s}}\right)=W_{\mathfrak{s p}_{\text {res }}(\mathcal{H})}$.

As the center $\mathfrak{z} \cong \mathbb{R}$ of $\widehat{\mathfrak{s p}}_{\text {res }}(\mathcal{H})$ acts by multiples of the identity on $S(\mathcal{H})$, we have $\mathfrak{z} \subseteq H\left(W_{\pi_{s}}\right)$, which finally leads to $W_{\pi_{s}}=q^{-1}\left(q\left(W_{\pi_{s}}\right)\right)=W_{\widehat{\mathfrak{s p}}_{\text {res }}}(\mathcal{H})$.

\subsection{The momentum set of the metaplectic representation}

Now that we have determined the cone $W_{\pi_{s}}$ for the metaplectic representation, we now apply the tools from Sec. 5 to determine the momentum set for the representation of the central extension $\widehat{\mathrm{HSp}}_{\text {res }}(\mathcal{H})$ acting on $S(\mathcal{H})$. In particular, we show that the momentum set is the closed convex hull of a single coadjoint orbit.

Definition 9.11. We define $\widehat{\mathrm{HSp}}_{\mathrm{res}}(\mathcal{H})$ as the quotient of the semidirect product

$$
\operatorname{Heis}(\mathcal{H}) \rtimes_{\alpha} \widehat{\operatorname{Sp}}_{\text {res }}(\mathcal{H}), \quad \alpha(g, u)((t, v)):=(t, g v),
$$

by the central subgroup

$$
S:=\left\{(t,(\mathbf{1}, z \mathbf{1})) \in \mathbb{R} \times Z: e^{i t} z=1\right\} \cong \mathbb{R}
$$

which acts trivially on $S(\mathcal{H})$. This means that $\widehat{\mathrm{HSp}}_{\text {res }}(\mathcal{H})$ is a central extension of $\mathcal{H} \rtimes \operatorname{Sp}_{\text {res }}(\mathcal{H})$ by $\mathbb{T}$. The corresponding Lie algebra cocycle is given by

$$
\left((v, x),\left(v^{\prime}, x^{\prime}\right)\right) \mapsto \operatorname{Im}\left\langle v, v^{\prime}\right\rangle+\eta\left(x, x^{\prime}\right)=\operatorname{Im}\left\langle v, v^{\prime}\right\rangle+\frac{1}{2} \operatorname{Im} \operatorname{tr}\left(x_{2} x_{2}^{\prime}\right)
$$

(cf. Theorem 9.3).

Remark 9.12. The representations $W$ of $\operatorname{Heis}(\mathcal{H})$ and $\pi_{s}$ of $\widehat{\operatorname{Sp}}_{\text {res }}(\mathcal{H})$ combine to a representation, also denoted $\pi_{s}$ of $\widehat{\mathrm{HSp}}_{\text {res }}(\mathcal{H})$ on $S(\mathcal{H})$. Since $(W, S(\mathcal{H}))$ is irreducible, the extension to $\widehat{\mathrm{HSp}}_{\text {res }}(\mathcal{H})$ is also irreducible. To see that it is smooth, it suffices to show that the function

$$
\operatorname{Heis}(\mathcal{H}) \rtimes \widehat{\operatorname{Sp}}_{\text {res }}(\mathcal{H}) \rightarrow \mathbb{C}, \quad(h, g) \mapsto\left\langle W(h) \pi_{s}(g) \Omega, \Omega\right\rangle=\left\langle\pi_{s}(g) \Omega, W(h)^{-1} \Omega\right\rangle
$$

is smooth (Theorem A.3). This follows from the smoothness of the vector $\Omega$ for Heis $(\mathcal{H})$ and $\widehat{\operatorname{Sp}}_{\text {res }}(\mathcal{H})($ cf. (30) and Theorem 9.3).

Proposition 9.13. The metaplectic representation $\left(\pi_{s}, S(\mathcal{H})\right)$ of $\widehat{\mathrm{HSp}}_{\mathrm{res}}(\mathcal{H})$ is semibounded with

$$
W_{\pi_{s}}=q^{-1}\left(\mathcal{H} \times W_{\mathfrak{s p}_{\text {res }}(\mathcal{H})}\right)=\operatorname{Ad}\left(\widehat{\operatorname{HSp}}_{\text {res }}(\mathcal{H})\right)\left(\mathfrak{z} \times C_{\mathfrak{u}(\mathcal{H})}\right),
$$

where $q: \widehat{\mathfrak{h} s p}_{\text {res }}(\mathcal{H}) \rightarrow \mathcal{H} \rtimes \mathfrak{s p}_{\text {res }}(\mathcal{H})$ is the quotient map whose kernel is the center $\mathfrak{z} \cong \mathbb{R}$. 
Proof. Let

$$
p: \mathfrak{h}_{\mathfrak{s}} \mathfrak{p}_{\text {res }}(\mathcal{H})=\mathfrak{h e i s}(\mathcal{H}) \rtimes \mathfrak{s p}_{\text {res }}(\mathcal{H}) \rightarrow \mathcal{H} \rtimes \mathfrak{s p}_{\text {res }}(\mathcal{H}), \quad(t, v, x) \mapsto(v, x)
$$

denote the quotient map, so that Theorem 6.7(iv) implies that

$$
W_{\mathfrak{h s p}_{\text {res }}(\mathcal{H})}=p^{-1}\left(W_{\mathfrak{s p}_{\text {res }}(\mathcal{H})}\right)=\operatorname{Ad}\left(\operatorname{HSp}_{\text {res }}(\mathcal{H})\right) W_{\mathfrak{s p}_{\text {res }}}(\mathcal{H}) .
$$

Modulo the center, this relation leads to

$$
\mathcal{H} \rtimes W_{\mathfrak{s p} \text { res }}(\mathcal{H})=\operatorname{Ad}\left(\mathcal{H} \rtimes \operatorname{Sp}_{\text {res }}(\mathcal{H})\right) W_{\mathfrak{s p} \text { res }}(\mathcal{H})=\operatorname{Ad}\left(\mathcal{H} \rtimes \operatorname{Sp}_{\text {res }}(\mathcal{H})\right) C_{\mathfrak{u}(\mathcal{H})}
$$

From Theorem 9.10 we know that $s_{\pi_{s}}(x)$ is finite if $x \in \mathfrak{z} \times C_{\mathfrak{u}(\mathcal{H})}$, so that $(40)$ implies that it is also finite if $q(x) \in \mathcal{H} \rtimes W_{\mathfrak{s p}_{\text {res }}}(\mathcal{H})$, i.e.

$$
W_{\pi_{s}} \supseteq q^{-1}\left(\mathcal{H} \rtimes W_{\mathfrak{s p} \text { res }}(\mathcal{H})\right) .
$$

This already proves that $\pi_{s}$ is semibounded because $W_{\pi_{s}}$ has interior points (Proposition 2.2). We further conclude that $\mathfrak{h e i s}(\mathcal{H}) \subseteq H\left(W_{\pi_{s}}\right)$, so that we obtain with Theorem 9.10 that

$$
\begin{aligned}
W_{\pi_{s}} & =\mathfrak{h e i s}(\mathcal{H})+\left(W_{\pi_{s}} \cap \widehat{\mathfrak{s p}}_{\mathrm{res}}(\mathcal{H})\right) \subseteq \mathfrak{h e i s}(\mathcal{H})+q^{-1}\left(W_{\mathfrak{s p}_{\mathrm{res}}(\mathcal{H})}\right) \\
& =q^{-1}\left(\mathcal{H} \times W_{\mathfrak{s p}_{\text {res }}(\mathcal{H})}\right) .
\end{aligned}
$$

This proves the desired equality.

Finally, we note that (40) implies that every element $x \in W_{\pi_{s}}$ is conjugate to an element $y \in q^{-1}\left(C_{\mathfrak{u}(\mathcal{H})}\right)$, which means that $y \in \mathfrak{z} \times C_{\mathfrak{u}(\mathcal{H})}$.

Theorem 9.14. The momentum set $I_{\pi_{s}}$ of the metaplectic representation of $\widehat{\mathrm{HSp}}_{\mathrm{res}}(\mathcal{H})$ is the closed convex hull of the coadjoint orbit of $\lambda:=\Phi_{\pi_{s}}([\Omega])$, and this linear functional is given by

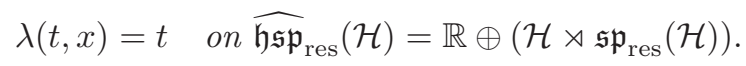

Proof. Since the metaplectic representation of $G:=\widehat{\mathrm{HSp}}_{\mathrm{res}}(\mathcal{H})$ is irreducible, we want to apply Theorem 5.11. For the subgroup $K:=\widehat{\mathrm{U}}(\mathcal{H}) \subseteq G$ with Lie algebra $\mathfrak{k}=\mathfrak{z} \oplus \mathfrak{u}(\mathcal{H})$, we have

$$
\mathfrak{g}_{\mathbb{C}} \cong \mathfrak{p}_{+} \oplus \mathfrak{k}_{\mathbb{C}} \oplus \mathfrak{p}_{-}, \quad \text { where } \mathfrak{p}_{ \pm}=\operatorname{ker}(\operatorname{ad} I \mp i \mathbf{1}) \oplus \operatorname{ker}(\operatorname{ad} I \mp 2 i \mathbf{1}),
$$

$I \in \mathfrak{u}(\mathcal{H})$ is the multiplication with $i$ on $\mathcal{H}$,

$$
\mathfrak{h e i s}(\mathcal{H})_{\mathbb{C}}=\mathfrak{z}_{\mathbb{C}} \oplus \mathcal{H}_{+} \oplus \mathcal{H}_{-}, \quad \mathcal{H}_{ \pm}:=\operatorname{ker}(\operatorname{ad} I \mp i \mathbf{1}) \subseteq \mathcal{H}_{\mathbb{C}}
$$

Since the Lie algebra $\mathfrak{k}$ of $K$ is complemented by the closed subspace $\mathcal{H} \oplus \mathfrak{p}_{2}$ in $\mathfrak{g}$, the coset space $G / K$ carries the structure of a Banach homogeneous space. Moreover, the closed $K$-invariant subalgebra $\mathfrak{k}_{\mathbb{C}} \oplus \mathfrak{p}_{+}$determines on $G / K$ the structure of a complex manifold for which the tangent space in the base point can be identified with $\mathfrak{g}_{\mathbb{C}} /\left(\mathfrak{k}_{\mathbb{C}}+\mathfrak{p}_{+}\right) \cong \mathfrak{p}_{-}([6$, Theorem 6.1] $)$.

Let $\Omega \in S^{0}(\mathcal{H}) \subseteq S(\mathcal{H})$ be the vacuum vector. Then $\Omega$ is a smooth vector (Remark 9.12), which is an eigenvector for $K$. On $S(\mathcal{H})$, the operator $-i \mathrm{~d} \pi(I)$ is 
diagonal, and $S^{n}(\mathcal{H})$ is the eigenspace corresponding to the eigenvalue $n$. As $-i$ ad $I$ acts on $\mathfrak{p}_{-}$with the eigenvalues -1 and -2 , it follows that $\mathrm{d} \pi_{s}\left(\mathfrak{p}_{-}\right) \Omega=\{0\}$, so that Theorem 5.11 provides a holomorphic equivariant map

$$
\eta: G / K \rightarrow \mathbb{P}\left(S(\mathcal{H})^{\prime}\right), \quad g \mapsto\left\langle\cdot, \pi_{s}(g)^{-1} \Omega\right\rangle
$$

and a realization of $\left(\pi_{s}, S(\mathcal{H})\right)$ by holomorphic sections of a complex line bundle over $G / K$. Since the representation $\left(\pi_{s}, S(\mathcal{H})\right)$ of $G$ is irreducible, $\Omega$ is a cyclic vector.

It remains to show that for each $x \in W_{\pi}$ the flow generated by $-x$ on $G / K$ extends holomorphically to $\mathbb{C}_{+}$. Since every such element $x$ is conjugate to an element of $\mathfrak{z} \times C_{\mathfrak{u}(\mathcal{H})}$, we may w.l.o.g. assume that $x \in C_{\mathfrak{u}(\mathcal{H})}$.

To get more information on $G / K$, we note that the choice of the complex structure implies that $G / K$ contains $\operatorname{Heis}(\mathcal{H}) /(K \cap \operatorname{Heis}(\mathcal{H})) \cong \mathcal{H}_{-} \cong \mathcal{H}^{\prime}$ as a complex submanifold. The translation action of $\operatorname{Heis}(\mathcal{H})$ by $(t, v) . z:=v+z$ on $\mathcal{H}$ factors through an action of the additive group $\mathcal{H}$ and extends naturally to a holomorphic action of the complexified group $\mathcal{H}_{\mathbb{C}} \cong \mathcal{H}_{+} \times \mathcal{H}_{-}$. As the $G$-action on $G / K$ induces a transitive action on the set of $\operatorname{Heis}(\mathcal{H})$-orbits on $G / K$, the action of $\operatorname{Heis}(\mathcal{H})$ extends to a holomorphic action of $\operatorname{Heis}(\mathcal{H})_{\mathbb{C}}$ on $G / K$. Therefore the action of the one-parameter group $t \mapsto \exp (-t x)$ extends to a holomorphic action of $\mathbb{C}_{+}$on $G / K$ if and only if the same holds for the action on the quotient space $\operatorname{Sp}_{\text {res }}(\mathcal{H}) / \mathrm{U}(\mathcal{H})$. As we have seen in Example 3.2(h), this space can be identified with the symmetric Hilbert domain

$$
\mathcal{D}_{s}=\left\{z \in B_{2}(\mathcal{H}): z^{\top}=z, z z^{*} \ll 1\right\},
$$

but here it is endowed with the opposite complex structure.

The group $\mathrm{U}(\mathcal{H})$ acts on $\mathcal{D}_{s}$ by $u . z=u z u^{\top}$, and for $x \in C_{\mathfrak{u}(\mathcal{H})}$ we obtain for our choice of complex structure the relation $e^{-i x} . z=e^{i x} v e^{i x^{\top}}$. Now $\left\|e^{i x}\right\|=\left\|e^{i x^{\top}}\right\|<1$ implies that the action of the one-parameter group $t \mapsto \exp (-t x)$ extends to $\mathbb{C}_{+}$, and this completes the proof.

Finally, we derive from Theorem 5.11(b) that $I_{\pi_{s}}=\overline{\operatorname{conv}}\left(\mathcal{O}_{\lambda}\right)$ holds for

$$
\lambda(x)=-i\left\langle\mathrm{~d} \pi_{s}(x) \Omega, \Omega\right\rangle .
$$

On $\mathfrak{k}=\widehat{\mathfrak{u}}(\mathcal{H})$ we have $\lambda(t, x)=t$, and $\lambda$ vanishes on $\mathfrak{p}_{\mathbb{C}}$ because $\mathfrak{p}_{-}$annihilates $\Omega$, and $\left\langle\mathrm{d} \pi_{s}\left(\mathfrak{p}_{+}\right) \Omega, \Omega\right\rangle=\left\langle\Omega, \mathrm{d} \pi_{s}\left(\mathfrak{p}_{-}\right) \Omega\right\rangle=0$. This completes the proof.

With similar and even easier arguments as in the proof of the preceding theorem, we also obtain:

Theorem 9.15. The momentum set $I_{\pi_{s}^{+}}$of the even metaplectic representation $\left(\pi_{s}^{+}, S^{\operatorname{even}}(\mathcal{H})\right)$ of $\widehat{\operatorname{Sp}}_{\text {res }}(\mathcal{H})$ is the closed convex hull of the coadjoint orbit of $\Phi_{\pi_{s}^{+}}([\Omega])$. 


\section{The Spin Representation}

In this section we take a closer look at the spin representation $\left(\pi_{a}, \Lambda(\mathcal{H})\right)$ of the central extension $\widehat{\mathrm{O}}_{\text {res }}(\mathcal{H})$ of $\mathrm{O}_{\text {res }}(\mathcal{H})$ on the fermionic Fock space $\Lambda(\mathcal{H})$. This representation arises from self-intertwining operators of the Fock representation of the $C^{*}$-algebra $\operatorname{CAR}(\mathcal{H})$. Here we also show that $\pi_{a}$ is semibounded and determine the corresponding cone $W_{\pi_{a}}$. For the irreducible representation of the identity component on the even part $\Lambda^{\text {even }}(\mathcal{H})$, we show that the momentum set is the weak-*-closed convex hull of a single coadjoint orbit.

\subsection{Semiboundedness of the spin representation}

Let $\mathcal{H}$ be a complex Hilbert space and write $\{a, b\}:=a b+b a$ for the anticommutator of two elements of an associative algebra. The $C A R$-algebra of $\mathcal{H}$ is a $C^{*}$-algebra $\operatorname{CAR}(\mathcal{H})$, together with a continuous antilinear map $a: \mathcal{H} \rightarrow \operatorname{CAR}(\mathcal{H})$ satisfying the canonical anticommutation relations

$$
\left\{a(f), a(g)^{*}\right\}=\langle g, f\rangle \mathbf{1} \quad \text { and } \quad\{a(f), a(g)\}=0 \quad \text { for } f, g \in \mathcal{H}
$$

and which is generated by the image of $a$. This determines $\operatorname{CAR}(\mathcal{H})$ up to natural isomorphism $\left(\left[14\right.\right.$, Theorem 5.2.8]). We also write $a^{*}(f):=a(f)^{*}$, which defines a complex linear map $a^{*}: \mathcal{H} \rightarrow \operatorname{CAR}(\mathcal{H})$.

The orthogonal group $\mathrm{O}(\mathcal{H})$ of the underlying real Hilbert space

$$
\left(\mathcal{H}_{\mathbb{R}}, \beta\right), \quad \beta(v, w)=\operatorname{Re}\langle v, w\rangle,
$$

acts by automorphisms on this $C^{*}$-algebra as follows. Writing a real linear isometry as $g=g_{1}+g_{2}$, where $g_{1}$ is linear and $g_{2}$ is antilinear, the relations $g g^{*}=g^{*} g=\mathbf{1}$ turn into

$$
g_{1} g_{1}^{*}+g_{2} g_{2}^{*}=\mathbf{1}=g_{1}^{*} g_{1}+g_{2}^{*} g_{2} \quad \text { and } \quad g_{1} g_{2}^{*}+g_{2} g_{1}^{*}=0=g_{2}^{*} g_{1}+g_{1}^{*} g_{2} .
$$

These relations imply that

$$
a_{g}: \mathcal{H} \rightarrow \operatorname{CAR}(\mathcal{H}), \quad f \mapsto a\left(g_{1} f\right)+a^{*}\left(g_{2} f\right)
$$

satisfies the same anticommutation relations, so that the universal property of $\operatorname{CAR}(\mathcal{H})$ implies the existence of a unique automorphism $\alpha_{g}$ with

$$
\alpha_{g}(a(f))=a_{g}(f) \quad \text { for } f \in \mathcal{H} .
$$

These automorphisms of the $\operatorname{CAR}(\mathcal{H})$ are called Bogoliubov automorphisms. They define an action of $\mathrm{O}(\mathcal{H})$ on $\operatorname{CAR}(\mathcal{H})$. In particular, the unitary group $\mathrm{U}(\mathcal{H}) \subseteq$ $\mathrm{O}(\mathcal{H})$ acts on $\operatorname{CAR}(\mathcal{H})$ by $\alpha_{g}(a(f))=a(g f)$ for $f \in \mathcal{H}$.

Remark 10.1. The $C^{*}$-algebra $\operatorname{CAR}(\mathcal{H})$ has a natural irreducible representation $\left(\pi_{0}, \Lambda(\mathcal{H})\right)$ on the antisymmetric Fock space $\Lambda(\mathcal{H})([14$, Proposition 5.2.2(3)]). The image $a_{0}(f):=\pi_{0}(a(f))$ acts by $a_{0}(f) \Omega=0$ and

$$
a_{0}(f)\left(f_{1} \wedge \cdots \wedge f_{n}\right)=\sum_{j=1}^{n}(-1)^{j-1}\left\langle f_{j}, f\right\rangle f_{1} \wedge \cdots \wedge f_{j-1} \wedge f_{j+1} \wedge \cdots \wedge f_{n}
$$


Accordingly, we have

$$
a_{0}^{*}(f) \Omega=f \quad \text { and } \quad a_{0}^{*}(f)\left(f_{1} \wedge \cdots \wedge f_{n}\right)=f \wedge f_{1} \wedge \cdots \wedge f_{n} .
$$

Let $I v=i v$ denote the complex structure on $\mathcal{H}$. The action of the restricted orthogonal group

$$
\mathrm{O}_{\text {res }}(\mathcal{H}):=\left\{g \in \mathrm{O}(\mathcal{H}):\|[g, I]\|_{2}<\infty\right\}
$$

on $\operatorname{CAR}(\mathcal{H})$ preserves the equivalence class of the representation $\pi_{0}$, so that there is a projective unitary representation $\pi_{a}: \mathrm{O}_{\text {res }}(\mathcal{H}) \rightarrow \mathrm{U}(\Lambda(\mathcal{H}))$, called the spin representation, satisfying

$$
\pi_{a}(g) \pi_{0}(A) \pi_{a}(g)^{*}=\pi_{0}\left(\alpha_{g} A\right) \quad \text { for } g \in \mathrm{O}_{\mathrm{res}}(\mathcal{H}), A \in \mathrm{CAR}(\mathcal{H})
$$

(cf. [77, Theorem 3, p. 35], [97]).

In analogy with Theorem 9.3 we here obtain:

Theorem 10.2. $\widehat{\mathrm{O}}_{\mathrm{res}}(\mathcal{H})$ is a Lie group and the spin representation is smooth. $A$ Lie algebra cocycle $\eta$ defining $\widehat{\mathfrak{o}}_{\text {res }}(\mathcal{H})$ as an extension of $\mathfrak{o}_{\text {res }}(\mathcal{H})$ by $\mathbb{R}$ is given by

$$
\eta(x, y)=-\frac{1}{2 i} \operatorname{tr}\left(\left[x_{2}, y_{2}\right]\right)
$$

Proof. With completely analogous arguments as in the proof of Theorem 9.3 we find with Appendix D that any $g \in \mathrm{O}_{\text {res }}(\mathcal{H})$ for which $g_{1}$ is invertible has a unique lift $\pi_{a}(g) \in \mathrm{U}(\Lambda(\mathcal{H}))$ with

$$
\pi_{a}(g) \Omega=c(g) e^{-\widehat{T}(g)} \quad \text { for } T(g):=g_{2} g_{1}^{-1} \in \operatorname{Aherm}(\mathcal{H})_{a}, c(g)=\left\|e^{-\widehat{T}(g)}\right\|^{-1}
$$

(cf. Lemma D.3).

This implies that $\widehat{\mathrm{O}}_{\text {res }}(\mathcal{H})$ is a Lie group, its representation $\pi_{a}$ on $\Lambda(\mathcal{H})$ is smooth, and that $\Omega$ is a smooth vector. Since $\mathrm{O}_{\text {res }}(\mathcal{H})$ acts smoothly on $\operatorname{CAR}(\mathcal{H})$, the space $\Lambda(\mathcal{H})^{\infty}$ of smooth vectors is invariant under $\pi_{0}(\operatorname{CAR}(\mathcal{H}))$, so that the irreducibility of the representation of $\operatorname{CAR}(\mathcal{H})$ on $\Lambda(\mathcal{H})$ implies the smoothness of $\pi_{a}$.

For the Lie algebra cocycle defining $\widehat{\mathfrak{o}}_{\text {res }}(\mathcal{H})$, we find as in the proof of Theorem 9.3 with Lemma D.3(iii)

$$
\eta(x, y)=2 \operatorname{Im}\left\langle\widehat{x}_{2}, \widehat{y}_{2}\right\rangle=-\operatorname{Im} \operatorname{tr}\left(x_{2} y_{2}\right)=-\frac{1}{2 i} \operatorname{tr}\left(\left[x_{2}, y_{2}\right]\right) .
$$

Remark 10.3. As in Sec. 9.2, we obtain a Banach-Lie group

$$
\mathrm{O}_{1,2}(\mathcal{H}):=\left\{g=g_{1}+g_{2} \in \mathrm{O}(\mathcal{H}):\left\|g_{2}\right\|_{2}<\infty,\left\|\mathbf{1}-g_{1}\right\|_{1}<\infty\right\}
$$

with

$$
\mathrm{O}_{\text {res }}(\mathcal{H}) \cong\left(\mathrm{O}_{1,2}(\mathcal{H}) \rtimes \mathrm{U}(\mathcal{H})\right) / N, \quad N \cong \mathrm{U}(\mathcal{H}) \cap \mathrm{O}_{1,2}(\mathcal{H})=\mathrm{U}_{1}(\mathcal{H})
$$


(cf. [63, Definition IV.7]). From [63, Remark IV.14] we recall that $\mathrm{O}_{\text {res }}(\mathcal{H})$ has two connected components and that its fundamental group is trivial. On the other hand,

$$
\pi_{0}\left(\mathrm{O}_{1,2}(\mathcal{H})\right) \cong \mathbb{Z} / 2 \mathbb{Z} \cong \pi_{1}\left(\mathrm{O}_{1,2}(\mathcal{H})\right)
$$

([81, Proposition 12.4.2], [63, Proposition III.15]), so that there exists a simply connected 2-fold covering group $q: \operatorname{Spin}_{1,2}(\mathcal{H}) \rightarrow \mathrm{O}_{1,2}(\mathcal{H})_{0}$ (cf. [33] for a discussion of the smaller group $\operatorname{Spin}_{1}(\mathcal{H})$ covering $\left.\operatorname{SO}_{1}(\mathcal{H})=\mathrm{O}_{1}(\mathcal{H})_{0}\right)$. As the inclusion $\mathrm{U}_{1}(\mathcal{H}) \rightarrow \mathrm{O}_{1,2}(\mathcal{H})$ induces a surjective homomorphism

$$
\pi_{1}\left(\mathrm{U}_{1}(\mathcal{H})\right) \cong \mathbb{Z} \rightarrow \pi_{1}\left(\mathrm{O}_{1,2}(\mathcal{H})\right) \cong \mathbb{Z} / 2,
$$

$\widehat{\mathrm{U}}_{1}(\mathcal{H}):=q^{-1}\left(\mathrm{U}_{1}(\mathcal{H})\right)$ is the unique 2-fold connected covering of $\mathrm{U}_{1}(\mathcal{H})$ from Sec. 9.2.

We further have an embedding

$$
\sigma: \mathfrak{o}_{1,2}(\mathcal{H}) \rightarrow \widehat{\mathfrak{o}}_{\text {res }}(\mathcal{H}), \quad \sigma(x):=\left(-\frac{1}{2 i} \operatorname{tr}\left(x_{1}\right), x\right)
$$

of Banach-Lie algebras. On the subgroup $\widehat{\mathrm{U}}_{1}(\mathcal{H}) \subseteq \operatorname{Spin}_{1,2}(\mathcal{H}), \sigma$ integrates to a group homomorphism $\sigma_{G}(g):=\left(g, \sqrt{\operatorname{det}}(g)^{-1} \pi_{s}(g)\right)$ (cf. Sec. 9.2) and $\sigma$ integrates to a morphism of Banach-Lie groups $\sigma_{G}: \operatorname{Spin}_{1,2}(\mathcal{H}) \rightarrow \widehat{\mathrm{O}}_{\text {res }}(\mathcal{H})$. Combining this map with the canonical inclusion $\mathrm{U}(\mathcal{H}) \hookrightarrow \widehat{\mathrm{O}}_{\text {res }}(\mathcal{H})$, the equivariance of $\sigma_{G}$ under conjugation with unitary operators implies the existence of a homomorphism

$$
\mu: \operatorname{Spin}_{1,2}(\mathcal{H}) \rtimes \mathrm{U}(\mathcal{H}) \rightarrow \widehat{\mathrm{O}}_{\mathrm{res}}(\mathcal{H}), \quad(g, u) \mapsto \sigma_{G}(g) u .
$$

The following proposition is proved as Proposition 9.5, using the representation $\pi_{a}$ instead of $\pi_{s}$.

Proposition 10.4. The homomorphism $\mu$ factors through an isomorphism

$$
\bar{\mu}:\left(\operatorname{Spin}_{1,2}(\mathcal{H}) \rtimes \mathrm{U}(\mathcal{H})\right) / \operatorname{ker} \mu \rightarrow \widehat{\mathrm{O}}_{\text {res }}(\mathcal{H})_{0}
$$

of connected Banach-Lie groups with $\operatorname{ker} \mu \cong \mathrm{SU}_{1}(\mathcal{H})$.

Remark 10.5. The embedding $\mathfrak{o}_{1,2}(\mathcal{H}) \hookrightarrow \widehat{\mathfrak{o}}_{\text {res }}(\mathcal{H})$ restricts in particular to an embedding $\mathfrak{o}_{1}(\mathcal{H}) \hookrightarrow \widehat{\mathfrak{o}}_{\text {res }}(\mathcal{H})$. One can show that the operators $\mathrm{d} \pi_{a}(x)$ are bounded for $x \in \mathfrak{o}_{1}(\mathcal{H})$ (cf. [2], [59, Sec. V]), so that we obtain a morphism of Banach-Lie algebras

$$
\mathfrak{o}_{1}(\mathcal{H}) \rightarrow \mathfrak{u}(\Lambda(\mathcal{H})), \quad x \mapsto \mathrm{d} \pi_{a}(x)-\frac{1}{2} \operatorname{tr}\left(x_{1}\right) \mathbf{1} .
$$

Definition 10.6. To determine the open cone $W_{\pi_{a}}$ for the spin representation, we have to take a closer look at natural cones in $\mathfrak{o}_{\text {res }}(\mathcal{H})$. Let $\mathfrak{u}_{\infty}(\mathcal{H})$ denote the ideal of compact skew-hermitian operators in $\mathfrak{u}(\mathcal{H})$ and recall the Calkin algebra $\operatorname{Cal}(\mathcal{H}):=$ $B(\mathcal{H}) / B_{\infty}(\mathcal{H})$, where $B_{\infty}(\mathcal{H})$ denotes the ideal of compact operators in the $C^{*}$ algebra $B(\mathcal{H})$. Then the surjection $B(\mathcal{H}) \rightarrow \operatorname{Cal}(B(\mathcal{H}))$ induces an isomorphism

$$
\mathfrak{u}(\mathcal{H}) / \mathfrak{u}_{\infty}(\mathcal{H}) \cong \mathfrak{u}(\operatorname{Cal}(\mathcal{H})) .
$$


Next we note that $\left\{x \in \mathfrak{o}_{\text {res }}(\mathcal{H}): x^{*}-x \in \mathfrak{u}_{\infty}(\mathcal{H})\right\}$ is a closed ideal of $\mathfrak{o}_{\text {res }}(\mathcal{H})$ which defines a quotient morphism $q: \mathfrak{o}_{\text {res }}(\mathcal{H}) \rightarrow \mathfrak{u}(\mathrm{Cal}(\mathcal{H}))$. We thus obtain an open invariant cone in $\mathfrak{o}_{\text {res }}(\mathcal{H})$ by

$$
W_{\mathfrak{o}_{\text {res }}(\mathcal{H})}:=q^{-1}\left(C_{\mathfrak{u}(\operatorname{Cal}(\mathcal{H}))}\right)=\left\{x \in \mathfrak{o}_{\text {res }}(\mathcal{H}):-i q(x) \ll 0\right\}
$$

(cf. Example 6.1). We write $W_{\widehat{\widehat{o}}_{\text {res }}(\mathcal{H})}$ for its inverse image in the central extension $\widehat{\mathfrak{o}}_{\text {res }}(\mathcal{H})$.

Theorem 10.7. The spin representation $\left(\pi_{a}, \Lambda(\mathcal{H})\right)$ of the connected Lie group $\widehat{\mathrm{O}}_{\mathrm{res}}(\mathcal{H})$ is semibounded with $W_{\pi_{a}}=W_{\widehat{\widehat{o}}_{\mathrm{res}}(\mathcal{H})}$.

Proof. First we note that under $\widehat{\mathrm{U}}(\mathcal{H}) \cong \mathbb{T} \times \mathrm{U}(\mathcal{H})$ the spin representation decomposes into the irreducible subspaces $\Lambda^{n}(\mathcal{H})$ with

$$
\pi_{a}(z, g)\left(v_{1} \wedge \cdots \wedge v_{n}\right)=z g v_{1} \wedge \cdots \wedge g v_{n} \quad \text { for } v_{1}, \ldots, v_{n} \in \mathcal{H} .
$$

If $i x \leq 0$, then the corresponding operator on $\Lambda^{n}(\mathcal{H}) \subseteq \mathcal{H}^{\otimes n}$ is also $\leq 0$, and this implies that

$$
s_{\pi_{a}}(t, x)=-t+s_{\pi_{a}}(x) \leq-t<\infty \quad \text { for } x \in C_{\mathfrak{u}(\mathcal{H})} .
$$

Writing $\mathfrak{z} \cong \mathbb{R}$ for the center of $\widehat{\mathfrak{o}}_{\text {res }}(\mathcal{H})$, we thus find that $\mathfrak{z} \times C_{\mathfrak{u}(\mathcal{H})} \subseteq B\left(I_{\pi_{a}}\right)$.

Next we consider the decomposition of $\mathfrak{g}=\widehat{\mathfrak{o}}_{\text {res }}(\mathcal{H})=\mathfrak{k} \oplus \mathfrak{p}_{2}$, where $\mathfrak{k}=\mathfrak{z} \times \mathfrak{u}(\mathcal{H})$ and $\mathfrak{p}_{2}=\left\{x \in \mathfrak{o}_{\text {res }}(\mathcal{H}): I x=-x I\right\}$. For the map

$$
F: \mathfrak{k} \times \mathfrak{p}_{2} \rightarrow \mathfrak{g}, \quad(x, y) \mapsto e^{\operatorname{ad} y} x \quad \text { we then have } \mathrm{d} F(x, 0)(a, b)=a+[b, x],
$$

which is invertible if and only if ad $x: \mathfrak{p}_{2} \rightarrow \mathfrak{p}_{2}$ is invertible. This is in particular the case for $x=I$, the complex structure of $\mathcal{H}$. Therefore the image of $\left(\mathfrak{z} \times C_{\mathfrak{u}(\mathcal{H})}\right) \times \mathfrak{p}_{2}$ has interior points, and since it is contained in the invariant subset $B\left(I_{\pi_{a}}\right)$, the cone $W_{\pi_{a}}=B\left(I_{\pi_{a}}\right)^{0}$ is non-empty. With Proposition 2.2 we now see that the representation $\left(\pi_{a}, \Lambda(\mathcal{H})\right)$ is semibounded.

To determine the cone $W_{\pi_{a}}$, we recall from Remark 10.5 that for each $x \in \mathfrak{o}_{1}(\mathcal{H})$, the corresponding operator $\mathrm{d} \pi_{a}(x)$ on $\Lambda(\mathcal{H})$ is bounded. This means that $\mathfrak{o}_{1}(\mathcal{H}) \subseteq$ $B\left(I_{\pi_{a}}\right) \subseteq \overline{W_{\pi_{a}}}$, and since $H\left(W_{\pi_{a}}\right)$ is a closed ideal (Lemma $2.9(\mathrm{v})$ ), it follows that

$$
\mathfrak{n}:=\mathfrak{z} \oplus \mathfrak{u}_{\infty}(\mathcal{H}) \oplus \mathfrak{p}_{2} \subseteq H\left(W_{\pi_{a}}\right) .
$$

In view of $\widehat{\mathfrak{o}}_{\text {res }}(\mathcal{H})=\mathfrak{u}(\mathcal{H})+\mathfrak{n}$, this proves that

$$
W_{\widehat{\mathfrak{o}}_{\mathrm{res}}(\mathcal{H})}=C_{\mathfrak{u}(\mathcal{H})}+\mathfrak{n} \subseteq W_{\pi_{a}}=\mathfrak{n}+\left(W_{\pi_{a}} \cap \mathfrak{u}(\mathcal{H})\right) .
$$

It therefore remains to show that

$$
W_{\pi_{a}} \cap \mathfrak{u}(\mathcal{H}) \subseteq C_{\mathfrak{u}(\mathcal{H})}+\mathfrak{u}_{\infty}(\mathcal{H}) .
$$

To verify this assertion, let $A=A^{*}$ be a hermitian operator on $\mathcal{H}$ and $\mathrm{d} \pi_{a}(A)$ denote the corresponding operator on $\Lambda^{n}(\mathcal{H})$. Suppose that there exists an $\varepsilon>0$ 
such that the range of the spectral projection $P([\varepsilon, \infty[)$ is infinite dimensional. Then there exists for each $n$ an orthonormal subset $v_{1}, \ldots, v_{n}$ in this space. We then have

$$
\begin{aligned}
& \left\langle\mathrm{d} \pi_{a}(A)\left(v_{1} \wedge \cdots \wedge v_{n}\right), v_{1} \wedge \cdots \wedge v_{n}\right\rangle \\
& \quad=\sum_{j=1}^{n}\left\langle v_{1} \wedge \cdots \wedge A v_{j} \wedge \cdots \wedge v_{n}, v_{1} \wedge \cdots \wedge v_{n}\right\rangle=\sum_{j=1}^{n}\left\langle A v_{j}, v_{j}\right\rangle \geq n \varepsilon,
\end{aligned}
$$

and this implies that $\mathrm{d} \pi_{a}(A)$ is not bounded from above. If, conversely, for every $\varepsilon>0$ the spectral projection $P([\varepsilon, \infty[)$ has finite dimensional range, then $A=$ $A_{-}+A_{+}$, where $A_{-} \leq 0$ and $A_{+}$is compact, i.e. $i A \in \overline{C_{\mathfrak{u}(\mathcal{H})}}+\mathfrak{u}_{\infty}(\mathcal{H})$. This implies that the open cone $W_{\pi_{a}} \cap \mathfrak{u}(\mathcal{H})$ is contained in $C_{\mathfrak{u}(\mathcal{H})}+\mathfrak{u}_{\infty}(\mathcal{H})$.

Remark 10.8. (a) With a similar argument as in Remark 9.4, one argues that under the action of the identity component $\widehat{\mathrm{O}}_{\text {res }}(\mathcal{H})_{0}$ on the space $\Lambda(\mathcal{H})$ decomposes into two irreducible subrepresentations $\Lambda^{\text {even }}(\mathcal{H})$ and $\Lambda^{\text {odd }}(\mathcal{H})$. However, the action of the full group $\widehat{\mathrm{O}}_{\text {res }}(\mathcal{H})$ is irreducible because the elements $g \in \widehat{\mathrm{O}}_{\text {res }}(\mathcal{H})$ not contained in the identity component exchange the two subspaces $\Lambda^{\text {even }}(\mathcal{H})$ and $\Lambda^{\text {odd }}(\mathcal{H})([81$, p. 239$])$.

(b) There is also an analog of the Banach-Lie algebra $\mathfrak{h s p}_{\text {res }}(\mathcal{H})$ acting irreducibly on $\Lambda(\mathcal{H})$ in the fermionic case. Here it is an infinite dimensional analog of the odd orthogonal Lie algebra $\mathfrak{s o}_{2 n+1}(\mathbb{R})$. To see this Lie algebra, we define linear and antilinear rank-one operators on $\mathcal{H}$ by

$$
P_{v, w}(x):=\langle x, w\rangle v \text { and } \bar{P}_{v, w}(x):=\langle w, x\rangle v
$$

and observe that $P_{v, w}^{*}=P_{w, v}$ and $\bar{P}_{v, w}^{*}=\bar{P}_{w, v}$. Therefore $Q_{v, w}:=P_{v, w}-P_{w, v} \in$ $\mathfrak{u}_{1}(\mathcal{H})$, and a direct calculation yields

$$
\mathrm{d} \pi_{a}\left(Q_{v, w}\right)=a_{0}^{*}(v) a_{0}(w)-a_{0}^{*}(w) a_{0}(v) .
$$

As $\operatorname{tr}\left(Q_{v, w}\right)=2 i \operatorname{Im}\langle v, w\rangle$, we obtain with the map $\sigma$ from (43) (Remark 10.3)

$$
\mathrm{d} \pi_{a}\left(\sigma\left(Q_{v, w}\right)\right)=\mathrm{d} \pi_{a}\left(-\operatorname{Im}\langle v, w\rangle, Q_{v, w}\right) .
$$

For the antilinear operators $\bar{Q}_{v, w}:=\bar{P}_{v, w}-\bar{P}_{w, v}$ we have $\bar{Q}_{v, w} \in \mathfrak{o}_{1}(\mathcal{H}) \subseteq$ $\mathfrak{o}_{1,2}(\mathcal{H})$ and

$$
\mathrm{d} \pi_{a}\left(\bar{Q}_{v, w}\right)=a_{0}^{*}(v) a_{0}^{*}(w)-a_{0}(w) a_{0}(v)=a_{0}^{*}(v) a_{0}^{*}(w)+a_{0}(v) a_{0}(w) .
$$

Next we observe that the operators $\rho(v):=\frac{1}{\sqrt{2}}\left(a_{0}(v)-a_{0}^{*}(v)\right)$ satisfy

$$
\begin{aligned}
{[\rho(v), \rho(w)] } & =\frac{1}{2}\left[a_{0}(v)-a_{0}^{*}(v), a_{0}(w)-a_{0}^{*}(w)\right] \\
& =\left(a_{0}(v) a_{0}(w)+a_{0}^{*}(v) a_{0}^{*}(w)\right)+\left(a_{0}^{*}(w) a_{0}(v)-a_{0}^{*}(v) a_{0}(w)\right)+i \operatorname{Im}\langle v, w\rangle \\
& =\mathrm{d} \pi_{a}\left(\operatorname{Im}\langle v, w\rangle, \bar{Q}_{v, w}-Q_{v, w}\right)=\mathrm{d} \pi_{a}\left(\sigma\left(\bar{Q}_{v, w}-Q_{v, w}\right)\right) .
\end{aligned}
$$

This calculation implies that we obtain on the direct sum $\mathcal{H} \oplus \mathfrak{o}_{1,2}(\mathcal{H})$ a Banach-Lie algebra structure with the bracket

$$
\left[(v, X),\left(v^{\prime}, X^{\prime}\right)\right]:=\left(X v^{\prime}-X^{\prime} v,\left[X, X^{\prime}\right]+\bar{Q}_{v, w}-Q_{v, w}\right),
$$


and

$$
(v, X) \mapsto \rho(v)+\mathrm{d} \pi_{a}(\sigma(X))
$$

defines a representation by unbounded operators on $\Lambda(\mathcal{H})$, where the subalgebra $\mathcal{H} \oplus \mathfrak{o}_{1}(\mathcal{H})$ is represented by bounded operators. A closer inspection shows that $\mathcal{H} \oplus \mathfrak{o}_{1}(\mathcal{H}) \cong \mathfrak{o}_{1}(\mathcal{H} \oplus \mathbb{R})$ is an infinite dimensional version of $\mathfrak{o}_{2 n+1}(\mathbb{R})$ (cf. [59, p. 215]).

Definition 10.9. Let $\mathcal{I}_{\beta} \subseteq \mathrm{GL}\left(\mathcal{H}_{\mathbb{R}}\right)$ be the set of orthogonal real-linear complex structures on the real Hilbert space $\mathcal{H}_{\mathbb{R}}$. This set parametrizes the complex Hilbert space structures on $\mathcal{H}_{\mathbb{R}}$ compatible with the given real Hilbert space structure.

Lemma 10.10. The following assertions hold:

(i) $\mathcal{I}_{\beta}=\mathrm{O}(\mathcal{H}) \cap \mathfrak{o}(\mathcal{H})=\left\{g \in \mathrm{GL}\left(\mathcal{H}_{\mathbb{R}}\right): g^{\top}=g^{-1}=-g\right\}$ is a submanifold of $\mathrm{O}(\mathcal{H})$.

(ii) The conjugation action of $\mathrm{O}(\mathcal{H})$ on $\mathcal{I}_{\beta}$ leads to a diffeomorphism $\mathcal{I}_{\beta} \cong$ $\mathrm{O}(\mathcal{H}) / \mathrm{U}(\mathcal{H})$

(iii) $\mathcal{I}_{\beta}^{\text {res }}:=\operatorname{Ad}\left(\mathrm{O}_{\text {res }}(\mathcal{H})\right) I=\left\{J \in \mathcal{I}_{\beta}:\|I-J\|_{2}<\infty\right\} \cong \mathrm{O}_{\text {res }}(\mathcal{H}) / \mathrm{U}(\mathcal{H})$.

Proof. (i) It only remains to show that $\mathcal{I}_{\beta}$ is a submanifold of $\mathrm{O}(\mathcal{H})$. For $J \in \mathcal{I}_{\beta}$ we parametrize a neighborhood of $J$ by the map $\mathfrak{o}(\mathcal{H}) \rightarrow \mathrm{O}(\mathcal{H}), x \mapsto J e^{x}$ which is a diffeomorphism on some open 0-neighborhood $U \subseteq \mathfrak{o}(\mathcal{H})$, which we may assume to be invariant under $\operatorname{Ad}(J)$. That $J e^{x}$ is a complex structure is equivalent to

$$
-J e^{x}=\left(J e^{x}\right)^{-1}=e^{-x} J^{-1}=-J e^{-J^{-1} x J},
$$

which is equivalent to $J x=-x J$. We conclude that $\mathcal{I}_{\beta}$ is a submanifold of $\mathrm{O}(\mathcal{H})$ whose tangent space can be identified with the set of $J$-antilinear elements in $\mathfrak{o}(\mathcal{H})$.

(ii) Let $I \in \mathcal{I}_{\beta}$ be the canonical complex structure given by $I v=i v$ and $J \in \mathcal{I}_{\beta}$. Then $\mathcal{H}=\left(\mathcal{H}_{\mathbb{R}}, I\right)$ and $\left(\mathcal{H}_{\mathbb{R}}, J\right)$ are two complex Hilbert spaces whose underlying real Hilbert spaces are isomorphic. This implies that they have the same complex Hilbert dimension, i.e. there exists a unitary isomorphism $g:\left(\mathcal{H}_{\mathbb{R}}, I\right) \rightarrow\left(\mathcal{H}_{\mathbb{R}}, J\right)$, i.e. $g \in \mathrm{O}(\mathcal{H})$ with $g I=J g$. Therefore $\mathrm{O}(\mathcal{H})$ acts transitively on $\mathcal{I}_{\beta}$ and the stabilizer of $I$ is the subgroup $\mathrm{U}(\mathcal{H})$ (cf. [5, Lemma 1]). Since its Lie algebra $\mathfrak{u}(\mathcal{H})$ is complemented by the closed subspace

$$
\mathfrak{p}:=\{x \in \mathfrak{o}(\mathcal{H}): I x=-x I\},
$$

it follows that $\mathrm{O}(\mathcal{H}) / \mathrm{U}(\mathcal{H})$ is a Banach homogeneous space diffeomorphic to $\mathcal{I}_{\beta}$.

(iii) For $g=g_{1}+g_{2} \in \mathrm{O}(\mathcal{H})$ the operator $[I, g]=\left[I, g_{2}\right]=2 I g_{2}$ is HilbertSchmidt if and only if $g_{2}$ is, i.e. $g \in \mathrm{O}_{\text {res }}(\mathcal{H})$. This in turn is equivalent to $g I g^{-1}-I=$ $[g, I] g^{-1}$ being Hilbert-Schmidt, so that

$$
\mathrm{O}_{\text {res }}(\mathcal{H})=\left\{g \in \mathrm{O}(\mathcal{H}):\left\|g I g^{-1}-I\right\|_{2}<\infty\right\}
$$

and therefore (ii) implies (iii). 
Remark 10.11. A priori, the $C^{*}$-algebra $\operatorname{CAR}(\mathcal{H})$ depends on the complex structure on $\mathcal{H}$, but it can also be expressed as the $C^{*}$-algebra generated by the hermitian elements $b(f):=\frac{1}{\sqrt{2}}\left(a(f)+a^{*}(f)\right)$ satisfying

$$
\{b(f), b(g)\}=\frac{1}{2}\left\{a(f), a^{*}(g)\right\}+\frac{1}{2}\left\{a^{*}(f), a(g)\right\}=\frac{1}{2}(\langle g, f\rangle+\langle f, g\rangle) \mathbf{1}=\beta(f, g) \mathbf{1} .
$$

In this sense it is the $C^{*}$-envelope of the Clifford algebra of $\left(\mathcal{H}_{\mathbb{R}}, \beta\right)$. From this point of view it is even more transparent why the group $\mathrm{O}(\mathcal{H})$ acts by automorphisms.

Now we can think of the Fock representation $\pi_{0}$ as depending on the complex structure $I$ on $\mathcal{H}$, and any other representation $\pi_{0} \circ \alpha_{g}$ is the Fock representation corresponding to the complex structure $g I g^{-1} \in \mathcal{I}_{\beta}$. We thus obtain a map of $\mathcal{I}_{\beta}$ into the set $\operatorname{Ext}(S(\mathrm{CAR}(\mathcal{H})))$ of pure states of $\operatorname{CAR}(\mathcal{H})$, mapping $J:=g I g^{-1}$ to $\omega_{J}(A):=\left\langle\pi_{0}\left(\alpha_{g}^{-1}(A)\right) \Omega, \Omega\right\rangle$. These states are called the Fock states of $\operatorname{CAR}(\mathcal{H})$, and in $[5$, Theorem 3$]$ they are essentially characterized as the pure quasi-free states (cf. Sec. 10.2 below).

Theorem 10.12. For the irreducible subrepresentation $\left(\pi_{a}^{+}, \Lambda^{\text {even }}(\mathcal{H})\right)$ of $\widehat{\mathrm{O}}_{\mathrm{res}}(\mathcal{H})$, the momentum set is the closed convex hull of the coadjoint orbit of $\lambda:=\Phi_{\pi_{a}}([\Omega])$. This functional is given by

$$
\lambda(t, x)=t \quad \text { on } \widehat{\mathfrak{o}}_{\text {res }}(\mathcal{H})=\mathbb{R} \oplus \mathfrak{o}_{\text {res }}(\mathcal{H}) .
$$

Proof. We want to apply Theorem 5.11. For the subgroup $K:=\widehat{\mathrm{U}}(\mathcal{H}) \subseteq G:=$ $\widehat{\mathrm{O}}_{\text {res }}(\mathcal{H})_{0}$ with Lie algebra $\mathfrak{k}=\mathfrak{z} \oplus \mathfrak{u}(\mathcal{H})$, we have

$$
\mathfrak{g}_{\mathbb{C}} \cong \mathfrak{p}_{+} \oplus \mathfrak{k}_{\mathbb{C}} \oplus \mathfrak{p}_{-}, \quad \text { where } \mathfrak{p}_{ \pm}=\operatorname{ker}(\operatorname{ad} I \mp 2 i \mathbf{1})
$$

and $I \in \mathfrak{u}(\mathcal{H})$ is the multiplication with $i$ on $\mathcal{H}$.

Since the Lie algebra $\mathfrak{k}$ of $K$ is complemented by the closed subspace $\mathfrak{p}_{2}$ of antilinear elements in $\mathfrak{g}$, the coset space $G / K$ carries the structure of a Banach homogeneous space (cf. Lemma 10.10). Moreover, the closed $K$-invariant subalgebra $\mathfrak{k}_{\mathbb{C}} \oplus \mathfrak{p}_{+}$determines on $G / K$ the structure of a complex manifold for which the tangent space in the base point can be identified with $\mathfrak{g}_{\mathbb{C}} /\left(\mathfrak{k}_{\mathbb{C}}+\mathfrak{p}_{+}\right) \cong \mathfrak{p}_{-}$ ([6, Theorem 6.1]).

In view of $(3)$, we obtain with [63, Proposition V.8, Remark V.10(c)] that the group $\mathrm{O}_{\text {res }}(\mathcal{H})$ acts transitively on the homogeneous space $\mathrm{O}_{\text {res }}\left(\mathcal{H}_{\mathbb{C}}, \beta_{\mathbb{C}}\right) / P$, where $P$ is the stabilizer of the subspace $\mathcal{H} \oplus\{0\} \subseteq \mathcal{H}_{\mathbb{C}}$ (cf. Example 3.2(d),(i)). From $P \cap \mathrm{O}_{\text {res }}(\mathcal{H})=\mathrm{U}(\mathcal{H})$ it now follows that the action of the Banach-Lie group $G$ on $G / K$ actually extends to a holomorphic action of a complex group.

Let $\Omega \in \Lambda^{0}(\mathcal{H})$ be the vacuum vector. Then $\Omega$ is a smooth vector which is an eigenvector for $K$. On $\Lambda(\mathcal{H})$, the operator $-i \mathrm{~d} \pi(I)$ is diagonal, and $\Lambda^{n}(\mathcal{H})$ is the eigenspace corresponding to the eigenvalue $n$. As $-i \operatorname{ad} I$ acts on $\mathfrak{p}_{-}$with the eigenvalue -2 , it follows that $\mathrm{d} \pi_{a}\left(\mathfrak{p}_{-}\right) \Omega=\{0\}$, so that Theorem 5.11 provides a holomorphic equivariant map

$$
\eta: G / K \rightarrow \mathbb{P}\left(\Lambda(\mathcal{H})^{\prime}\right), \quad g \mapsto\left\langle\cdot, \pi_{a}(g)^{-1} \Omega\right\rangle
$$


and a realization of $\left(\pi_{a}, \Lambda^{\text {even }}(\mathcal{H})\right)$ by holomorphic sections of a complex line bundle over $G / K$. Since the representation $\left(\pi_{a}, \Lambda^{\text {even }}(\mathcal{H})\right)$ of $G$ is irreducible, $\Omega$ is a cyclic vector. As we have argued above, the action of every one-parameter subgroup of $G$ extends to a holomorphic action of $\mathbb{C}$ on $G / K$, so that Theorem $5.11(\mathrm{c})$ implies that $I_{\pi}$ is the closed convex hull of the coadjoint orbit of $\lambda$. That $\lambda$ has the desired form follows as in the proof of Theorem 9.14.

\subsection{Quasi-free representations}

Let $P=P^{*}=P^{2}$ be an orthogonal projection on $\mathcal{H}, \mathcal{H}_{-}:=\operatorname{im} P, \mathcal{H}_{+}:=\operatorname{ker} P$, and $\Gamma: \mathcal{H} \rightarrow \mathcal{H}$ be an isometric antilinear involution commuting with $P$. Then $\tau_{P}:=(\mathbf{1}-P)+\Gamma P \in \mathrm{O}(\mathcal{H})$. We write $a_{0}: \mathcal{H} \rightarrow B(\Lambda(\mathcal{H}))$ for the map corresponding to the Fock representation of $\operatorname{CAR}(\mathcal{H})$ (Remark 10.1). Twisting with the Bogoliubov automorphism defined by $\tau_{P}$, we obtain an irreducible representation $\left(\pi_{P}, \Lambda(\mathcal{H})\right)$ of $\operatorname{CAR}(\mathcal{H})$ by

$$
a_{P}(f):=a_{0}((\mathbf{1}-P) f)+a_{0}^{*}(\Gamma P f)=\pi_{0}\left(a_{\tau_{P}}(f)\right) \text { for } f \in \mathcal{H},
$$

i.e. $\pi_{P}=\pi_{0} \circ \alpha_{\tau_{P}}$. These representations are called quasi-free. For $P=0$, we recover the Fock representation defined by $a_{0}$. Two quasi-free representations $a_{P}$ and $a_{Q}$ are equivalent if and only if $\|P-Q\|_{2}<\infty$ ([80]).

Remark 10.13. The physical interpretation of $P$ is that its range $\mathcal{H}_{-}$consists of the negative energy states and its kernel $\mathcal{H}_{+}$consists of the positive energy states. For $f \in \mathcal{H}_{+}$we have $a_{P}(f) \Omega=0$ and likewise $a_{P}^{*}(f) \Omega=0$ for $f \in \mathcal{H}_{-}$. This is interpreted in such a way that the creation operator $a_{P}^{*}(f)$ cannot create an additional negative state from $\Omega$ because all negative states are already filled (Pauli's Principle). Likewise, the annihilation operator $a_{P}(f)$, corresponding to the positive energy vector $f$, cannot extract any positive energy state from $\Omega$.

The restricted unitary group $\mathrm{U}_{\text {res }}(\mathcal{H}, P)$ (cf. Example $3.2(\mathrm{c})$ ) is a subgroup of $\mathrm{O}(\mathcal{H})$, and for all projections $Q=g P g^{-1}, g \in \mathrm{U}_{\text {res }}(\mathcal{H}, P)$ we have $\|P-Q\|_{2}<\infty$, so that the equivalence of $a_{P}$ and $a_{Q}$ leads to a projective unitary representation of $\mathrm{U}_{\text {res }}(\mathcal{H})$ on $\Lambda(\mathcal{H})$ determined by

$$
\pi_{a}^{P}(g) a_{P}(f) \pi_{a}^{P}(g)^{*}=a_{P}(g f), \quad f \in \mathcal{H}, g \in \mathrm{U}_{\text {res }}(\mathcal{H}) .
$$

Let $\widehat{\mathrm{U}}_{\text {res }}(\mathcal{H})$ denote the corresponding central extension and write $\pi_{a}^{P}$ for its unitary representation on $\Lambda(\mathcal{H})$. To see that we thus obtain a semibounded representation of a Lie group, we first note that we have an embedding

$$
\iota: \mathrm{U}(\mathcal{H}) \rightarrow \mathrm{O}(\mathcal{H}), \quad g \mapsto \tau_{P} g \tau_{\mathrm{P}}
$$

for which $\mathrm{U}_{\text {res }}(\mathcal{H}, P)$ is precisely the inverse image of $\mathrm{O}_{\text {res }}(\mathcal{H})$ and that for $g \in$ $\mathrm{U}_{\text {res }}(\mathcal{H}, P)$ we have

$$
\begin{aligned}
\pi_{a}\left(\tau_{P} g \tau_{P}\right) a_{P}(f) \pi_{a}\left(\tau_{P} g \tau_{P}\right)^{*} & =\pi_{a}\left(\tau_{P} g \tau_{P}\right) \pi_{0}\left(\alpha_{\tau_{P}} a(f)\right) \pi_{a}\left(\tau_{P} g \tau_{P}\right)^{*} \\
& =\pi_{0}\left(\alpha_{\tau_{P} g} a(f)\right)=\pi_{P}\left(a_{g}(f)\right)=a_{P}(g f),
\end{aligned}
$$


so that the projective representation of $\mathrm{U}_{\text {res }}(\mathcal{H}, P)$ on $\Lambda(\mathcal{H})$ coincide with $\pi_{a} \circ \iota$ (cf. [77, p. 53]). It follows in particular that $\widehat{\mathrm{U}}_{\text {res }}(\mathcal{H}, P) \cong \iota^{*} \widehat{\mathrm{O}}_{\text {res }}(\mathcal{H})$ is a Lie group, $\pi_{a}^{P}$ is a smooth representation, and since $\operatorname{im}(\mathbf{L}(\iota))$ intersects the cone $C_{\mathfrak{u}(\mathcal{H})} \subseteq W_{\pi_{a}}$, the representation $\pi_{a}^{P}$ is semibounded (cf. Proposition 4.7(iv)). More precisely, each element $x=\left(x_{+}, x_{-}\right) \in \mathfrak{u}\left(\mathcal{H}_{+}\right) \oplus \mathfrak{u}\left(\mathcal{H}_{-}\right)$with $i x_{+} \ll 0$ and $i x_{-} \gg 0$ lies in $W_{\pi_{a}^{P}}$. This holds in particular for $\tau_{p} I \tau_{p}=I(\mathbf{1}-2 P)$.

In the physics literature, the corresponding self-adjoint operator

$$
Q:=-i \mathrm{~d} \pi_{a}^{P}(I)=\mathrm{d} \pi_{a}(\mathbf{1}-2 P)
$$

is called the charge operator and $\mathbf{1}-2 P$ the one-particle charge operator. Its 1-eigenspace is $\mathcal{H}_{+}$and its -1-eigenspace is $\mathcal{H}_{-}$.

Remark 10.14. The situation described above can be viewed as a "second quantization" procedure that can be used to turn a self-adjoint operator $A$ on the single particle space $\mathcal{H}$ into a non-negative operator $\widehat{A}$ on the many-particle space. In fact, let $P:=P(]-\infty, 0[)$ denote the spectral projection of $A$ corresponding to the open negative axis. Then $i A$ generates a strongly continuous one-parameter group $\gamma_{A}(t):=e^{i t A}$ of $\mathrm{U}\left(\mathcal{H}_{+}\right) \times \mathrm{U}\left(\mathcal{H}_{-}\right) \subseteq \mathrm{U}_{\text {res }}(\mathcal{H}, P)$, and $\pi_{a}^{P}\left(\gamma_{A}(t)\right)=e^{i t \widehat{A}}$ is a one-parameter group of $\mathrm{U}(\Lambda(\mathcal{H}))$ whose infinitesimal generator $\widehat{A}$ has non-negative spectrum.

For more details on the complex manifolds $\mathrm{O}_{\text {res }}(\mathcal{H}) / \mathrm{U}(\mathcal{H})$ (the isotropic restricted Grassmannian) and $\mathrm{U}_{\text {res }}(\mathcal{H}, P) /\left(\mathrm{U}(P(\mathcal{H})) \times \mathrm{U}\left(\mathcal{H}_{+}\right)\right)$(the restricted or Sato-Segal-Wilson Grassmannian) we refer to [81] and [98], where one also finds a detailed discussion of the corresponding complex line bundles, the Pfaffian line bundle Pf over $\mathrm{O}_{\text {res }}(\mathcal{H}) / \mathrm{U}(\mathcal{H})$ whose dual permits the even spin representation as a space of holomorphic sections, and the determinant line bundle Det over the restricted Grassmannian whose dual provides a realization of the representations of $\widehat{\mathrm{U}}_{\text {res }}(\mathcal{H}, P)$ mentioned above. Physical aspects of highest weight representations of $\mathrm{U}_{\text {res }}(\mathcal{H})$ are discussed in [17].

\section{Perspectives}

Classification problems. In the preceding sections we mainly discussed three prototypical classes of semibounded representations: highest weight representations of the Virasoro group, the metaplectic representation on the bosonic Fock space and the spin representation on the fermionic Fock space.

These representations are of fundamental importance in mathematical physics and homomorphisms from a Lie group $G$ to $\operatorname{Sp}_{\text {res }}(\mathcal{H}), \mathrm{O}_{\text {res }}(\mathcal{H})$ or $\mathrm{U}_{\text {res }}(\mathcal{H}, P)$ can be used to obtain semibounded representations of a central extension by pulling back the representations discussed above. This construction has been a major source of (projective) representations for loop groups $G=C^{\infty}\left(\mathbb{S}^{1}, K\right)$ and $\operatorname{Diff}\left(\mathbb{S}^{1}\right)$ (cf. $[88,81,15,17])$. 
What still remains to be developed is a better global perspective on semibounded representations, including classification results on irreducible ones and the existence of direct integral decompositions.

Problem 11.1. Classify all irreducible semibounded unitary representations of the groups $\widehat{\operatorname{Sp}}_{\text {res }}(\mathcal{H})$, $\widehat{\mathrm{O}}_{\text {res }}(\mathcal{H})$ and $\widehat{\mathrm{U}}_{\mathrm{res}}(\mathcal{H}, P)$, where $\mathcal{H}$ is a complex Hilbert space and $P$ an orthogonal projection on $\mathcal{H}$.

These classification problems are special cases of the more general problem of the classification of the projective semibounded unitary representations of the automorphism groups of hermitian Hilbert symmetric spaces. For the three groups above, the corresponding spaces of $\mathcal{I}_{\omega}^{\text {res }}, \mathcal{I}_{\beta}^{\text {res }}$, resp., the restricted Grassmannian $\operatorname{Gr}_{\text {res }}(\mathcal{H}, P):=\left\{g P g^{-1}: g \in \mathrm{U}_{\text {res }}(\mathcal{H}, P)\right\}$. A crucial difference between these spaces is that the first one is equivalent to a symmetric Hilbert domain (of negative curvature) and the latter two are positively curved spaces. This difference is also reflected in the difference between the cones $W_{\pi_{s}}$ and $W_{\pi_{s}}$ in $\widehat{\mathfrak{s p}}_{\mathrm{res}}(\mathcal{H})$ and $\widehat{\mathfrak{o}}_{\mathrm{res}}(\mathcal{H})$ (Theorems 9.10 and 10.7).

An even larger class of groups arises as automorphism groups of Hilbert flag manifolds such as the orbit of a finite flag in $\mathcal{H}$ under the group $\mathrm{U}_{2}(\mathcal{H})$. For a systematic discussion of these manifolds and the topology of the corresponding real and complex groups we refer to $[63$, Sec. V] and for corresponding representations to $[65]$.

For loop groups of the form $C^{\infty}\left(\mathbb{S}^{1}, K\right), K$ a compact connected Lie group, the irreducible projective positive energy representations can be identified as highest weight representations ([81, Theorem 11.2.3], [62, Corollary VII.2], and in particular [88, Proposition 3.1] for $K=\mathbb{T}$ ). With the convexity theorems in [3] and [40] it should be possible to show that these representations are semibounded and one can hope for an analog of Theorem 8.22 asserting that every irreducible semibounded representation of the corresponding double extension either is a highest weight representation or its dual. In [69] it is shown on the algebraic level that there also exist many interesting unitary representations of infinite rank generalizations of twisted and untwisted loop algebras, resp., their double extensions (so-called locally affine Lie algebras). On the group level they correspond to (double extensions of) groups of the form $C^{\infty}\left(\mathbb{S}^{1}, K\right)$, where $K$ is a Hilbert-Lie group, such as $\mathrm{U}_{2}(\mathcal{H})$. It seems quite likely that all these representations are semibounded with momentum sets generated by a single coadjoint orbit.

New sources of semibounded representations. Although many interesting classes of semibounded representations are known, a systematic understanding of the geometric sources of these representations is still lacking. As we have seen above, on the Lie algebra level the existence of open invariant cones in a trivial central extension is necessary (Remark 4.8). However, as the example $\mathcal{V}\left(\mathbb{S}^{1}\right)$ shows, it is not sufficient (Theorem 8.7). 
Clearly, the circle $\mathbb{S}^{1}$ plays a special role in many constructions, as the rich theory for central extensions of loop groups and the Virasoro group shows. Beyond $\mathbb{S}^{1}$, it seems that Lie algebras of conformal vector fields (as generalizations of $\mathcal{V}\left(\mathbb{S}^{1}\right)$ ) (cf. Example 6.13, [51] and [93]) and Lie algebras of sections of vector bundles over Lorentzian manifolds (or more general "causal" spaces, [38]) are natural candidates to be investigated with respect to the existence of semibounded representations. The latter class of Lie algebras is a natural generalization of loop algebras. Here an interesting point is that, although the conformal groups $\operatorname{Conf}\left(\mathbb{S}^{1}\right)=\operatorname{Diff}\left(\mathbb{S}^{1}\right)$ of the circle and the conformal group $\operatorname{Conf}\left(\mathbb{S}^{1,1}\right)$ of the Lorentzian torus are infinite dimensional, for Lorentzian manifolds of dimension $\geq 3$, the conformal groups are finite dimensional (cf. [86]). In particular, it is contained in the list of [51]. This leads to the well-studied class of hermitian Lie groups (see in particular $[93,95,38]$ ).

Coadjoint orbits. There is a symplectic version of semibounded representations, namely Hamiltonian actions $\sigma: G \times M \rightarrow M$ with a momentum map $\Phi: M \rightarrow \mathfrak{g}^{\prime}$ for which the image of the momentum map is semi-equicontinuous. If $G$ is finite dimensional and $\Phi(M)$ is closed, then Proposition 2.7 implies that there exists an $x \in \mathfrak{g}$ for which the Hamiltonian function $H_{x}(m):=\Phi(m)(x)$ is proper. In particular, $\Phi$ is a proper map. In this sense the semi-equicontinuity of the image of $\Phi$ is a weakening of properness, which is a useful property as far as convexity properties are concerned ([37]).

Even though we do not know in general to which extent coadjoint orbits of infinite dimensional Lie groups are manifolds, the orbit $\mathcal{O}_{\lambda}$ can always be viewed as the range of a momentum map $\Phi: G \rightarrow \mathfrak{g}^{\prime}, g \mapsto \mathrm{Ad}^{*}(g) \lambda$ corresponding to the left action of $G$ on itself preserving the left invariant closed 2 -form $\Omega$ with $\Omega_{1}(x, y)=$ $\lambda([x, y])$. This action is semibounded if and only if $\mathcal{O}_{\lambda}$ is semi-equicontinuous.

Since the momentum sets of semibounded representations always consist of semi-equicontinuous orbits, the identification of the set $\mathfrak{g}_{\text {seq }}^{\prime}$ of semi-equicontinuous coadjoint orbits of a given Lie algebra is already a solid first step towards the understanding of corresponding semibounded representations. As we have seen in many situations above, a useful tool to study convexity properties of coadjoint orbits are projection maps $p_{\mathfrak{t}}: \mathfrak{g} \rightarrow \mathfrak{t}$, where $\mathfrak{t} \subseteq \mathfrak{g}$ is a "compactly embedded" subalgebra, i.e. the action of $e^{\text {adt }}$ on $\mathfrak{g}$ factor through the action of a compact Abelian group. As we have seen in Sec. 9, sometimes one does not want to project to Abelian subalgebras and one has to study projections $p_{\mathfrak{k}}: \mathfrak{g} \rightarrow \mathfrak{k}$, where $\mathfrak{k}$ is a subalgebra for which $e^{\text {ad } \mathfrak{k}}$ leaves a norm on $\mathfrak{g}$ invariant.

This is of particular interest to understand open invariant cones $W \subseteq \mathfrak{g}$ because they often have the form

$$
W=\operatorname{Ad}(G)(W \cap \mathfrak{k}) \quad \text { with } p_{\mathfrak{k}}(W)=W \cap \mathfrak{k}
$$

(cf. Theorem 6.7). In this situation on needs convexity theorems of the type

$$
p_{\mathfrak{k}}\left(\mathcal{O}_{x}\right) \subseteq \overline{\operatorname{conv}}\left(\operatorname{Ad}\left(N_{G}(\mathfrak{k})\right) x\right)+C,
$$


where $C$ is a certain invariant convex cone in $\mathfrak{k}$ and $N_{G}(\mathfrak{k}) \subseteq G$ is the normalizer of $\mathfrak{k}$ in $G$ (cf. [60] for finite dimensional Lie algebras). If $\mathfrak{k}=\mathfrak{t}$ is Abelian, then $N_{G}(\mathfrak{t})$ is an analog of the Weyl group.

For infinite dimensional Lie algebras, not many convexity theorems are known. Of relevance for semibounded representations is the particular case of affine KacMoody Lie algebras $([3,40])$, and for Lie algebras of bounded operators on Hilbert spaces the infinite dimensional version of Kostant's Theorem by A. Neumann ([75]) is crucial. What is still lacking is a uniform framework for results of this type.

\section{A. Smooth Vectors for Representations}

Let $G$ be a Lie group with Lie algebra $\mathfrak{g}$ and $\operatorname{exponential~function~} \exp _{G}: \mathfrak{g} \rightarrow G$. Further, let $V$ be a locally convex space and $\pi: G \rightarrow \mathrm{GL}(V)$ be a homomorphism defining a continuous action of $G$ on $V$. We write $\pi^{v}(g):=\pi(g) v$ for the orbit maps and

$$
V^{\infty}:=\left\{v \in V: \pi^{v} \in C^{\infty}(G, V)\right\}
$$

for the space of smooth vectors. In this Appendix we collect some results of [71] that are used in the present paper. Let

$$
\mathrm{d} \pi: \mathfrak{g} \rightarrow \operatorname{End}\left(V^{\infty}\right), \quad \mathrm{d} \pi(x) v:=\left.\frac{d}{d t}\right|_{t=0} \pi(\exp t x) v
$$

denote the derived action of $\mathfrak{g}$ on $V^{\infty}$. That this is indeed a representation of $\mathfrak{g}$ follows by observing that the map $V^{\infty} \rightarrow C^{\infty}(G, V), v \mapsto \pi^{v}$ intertwines the action of $G$ with the right translation action on $C^{\infty}(G, V)$, and this implies that the derived action corresponds to the action of $\mathfrak{g}$ on $C^{\infty}(G, V)$ by left invariant vector fields (cf. [62, Remark IV.2] for details).

Definition A.1. Let $G$ be a Banach-Lie group and write $\mathcal{P}(V)$ for the set of continuous seminorms on $V$. For each $p \in \mathcal{P}(V)$ and $n \in \mathbb{N}_{0}$ we define a seminorm $p_{n}$ on $V^{\infty}$ by

$$
p_{n}(v):=\sup \left\{p\left(\mathrm{~d} \pi\left(x_{1}\right) \cdots \mathrm{d} \pi\left(x_{n}\right) v\right):\left\|x_{i}\right\| \leq 1\right\}
$$

and endow $V^{\infty}$ with the locally convex topology defined by these seminorms.

Theorem A.2. If $(\pi, V)$ is a representation of the Banach-Lie group $G$ on the locally convex space $V$ defining a continuous action of $G$ on $V$, then the action $\sigma(g, v):=\pi(g) v$ of $G$ on $V^{\infty}$ is smooth. If $V$ is a Banach space, then $V^{\infty}$ is complete, i.e. a Fréchet space.

Theorem A.3. If $(\pi, \mathcal{H})$ is a unitary representation of a Lie group $G$, then $v \in \mathcal{H}$ is a smooth vector if and only if the corresponding matrix coefficient $\pi^{v, v}(g):=$ $\langle\pi(g) v, v\rangle$ is smooth on a 1-neighborhood in $G$. If, in addition, $v$ is cyclic, then the representation is smooth. 
In the following we write $\bar{u}$ for the image of a unitary operator $u \in \mathrm{U}(\mathcal{H})$ in the projective unitary group $\mathrm{PU}(\mathcal{H}):=\mathrm{U}(\mathcal{H}) / \mathbb{T} \mathbf{1}$.

Theorem A.4. Let $G$ be a connected Lie group, $\mathcal{H}$ a complex Hilbert space and $\pi: G \rightarrow \mathrm{U}(\mathcal{H})$ be a map with $\pi(\mathbf{1})=\mathbf{1}$ for which the corresponding map $\bar{\pi}: G \rightarrow \mathrm{PU}(\mathcal{H})$ is a group homomorphism.

If there exists a $v \in \mathcal{H}$ for which the function $(g, h) \mapsto\langle\pi(g) \pi(h) v, v\rangle$ is smooth on a neighborhood of $(\mathbf{1}, \mathbf{1})$ in $G \times G$, then the central extension

$$
\widehat{G}:=\bar{\pi}^{*} \mathrm{U}(\mathcal{H})=\{(g, u) \in G \times \mathrm{U}(\mathcal{H}): \bar{\pi}(g)=\bar{u}\}
$$

of $G$ by $\mathbb{T}$ is a Lie group and $v$ is a smooth vector for the representation $(\widehat{\pi}, \mathcal{H})$ of $\widehat{G}$ by $\widehat{\pi}(g, u):=u$.

Proof. To exhibit $\widehat{G}$ as a Lie group, we have to show that there exists a section $\sigma: G \rightarrow \widehat{G}$ for which the corresponding 2-cocycle

$$
f_{\sigma}\left(g_{1}, g_{2}\right)=\sigma\left(g_{1}\right) \sigma\left(g_{2}\right) \sigma\left(g_{1} g_{2}\right)^{-1}
$$

is smooth in a neighborhood of $(\mathbf{1}, \mathbf{1})$ ([64, Proposition 4.2]). Here we use that $G$ is connected. A particular section $\sigma: G \rightarrow \widehat{G}$ is given by $\sigma(g)=(g, \pi(g))$.

Let $U \subseteq G$ be an open 1-neighborhood such that

$$
\left\langle\pi(g) v, \pi(h)^{-1} v\right\rangle \neq 0 \quad \text { for } g, h \in U .
$$

Its existence follows from our continuity assumption. If $U^{\prime} \subseteq U$ is an open 1neighborhood with $U^{\prime} U^{\prime} \subseteq U$, we then have for $g_{1}, g_{2} \in U^{\prime}$

$$
f_{\sigma}\left(g_{1}, g_{2}\right)\left\langle\pi\left(g_{1} g_{2}\right) v, v\right\rangle=\left\langle\pi\left(g_{1}\right) \pi\left(g_{2}\right) v, v\right\rangle,
$$

which leads to

$$
f_{\sigma}\left(g_{1}, g_{2}\right)=\frac{\left\langle\pi\left(g_{1}\right) \pi\left(g_{2}\right) v, v\right\rangle}{\left\langle\pi\left(g_{1} g_{2}\right) v, v\right\rangle} .
$$

Therefore $f_{\sigma}$ is smooth in a neighborhood of $(\mathbf{1}, \mathbf{1})$. This shows that $\widehat{G}$ is a Lie group and the multiplication map $G \times \mathbb{T} \rightarrow \widehat{G},(g, t) \mapsto(g, t \pi(g))$ is smooth in a neighborhood of $\mathbf{1}$ ([64, Proposition 4.2]). The representation $\widehat{\pi}$ now satisfies

$$
\langle\widehat{\pi}(g, t \pi(g)) v, v\rangle=\langle t \pi(g) v, v\rangle=t\langle\pi(g) v, v\rangle,
$$

which is smooth in a neighborhood of $\mathbf{1}$. Now Theorem A.3 implies that $v$ is a smooth vector for the representation $\widehat{\pi}$.

Remark A.5. The assumption that $G$ is connected in the preceding theorem can be removed if $G$ is a Banach-Lie group. In this case we assume, in addition, that $\bar{\pi}$ is continuous in the sense that all functions $g \mapsto|\langle\pi(g) v, w\rangle|$ on $G$ are continuous (cf. [52, p. 175]). Then $\widehat{G}$ is the pullback of a central extension of topological groups $\mathrm{U}(\mathcal{H}) \rightarrow \mathrm{PU}(\mathcal{H})$, hence in particular a topological group with respect to the topology inherited from $G \times \mathrm{U}(\mathcal{H})$. Under the assumptions of Theorem A.4, 
the central extension $\widehat{G}_{0}$ of the identity component $G_{0}$ of $G$ carries a natural Lie group structure compatible with the given topology on $\widehat{G}$. For each $g \in \widehat{G}$, the conjugation map $c_{g}$ induces a continuous automorphism of the Banach-Lie group $\widehat{G}_{0}$, and since continuous homomorphisms of Banach-Lie groups are automatically smooth ([66, Theorem IV.1.18]), $c_{g}$ also defines a Lie automorphism of $\widehat{G}_{0}$. This implies that $\widehat{G}$ carries a unique Lie group structure which coincides on the open subgroup $\widehat{G}_{0}$ with the given one (see also [64, Remark 4.3]).

Remark A.6. In the situation of Theorem A.4, the Lie algebra cocycle defining the central extension $\widehat{\mathfrak{g}}=\mathbf{L}(\widehat{G})$ of $\mathfrak{g}$ by $\mathbb{R}$ in the sense that

$$
\widehat{\mathfrak{g}}=\mathbb{R} \oplus_{\eta} \mathfrak{g}, \quad\left[(t, x),\left(t^{\prime}, x^{\prime}\right)\right]=\left(\eta\left(x, x^{\prime}\right),\left[x, x^{\prime}\right]\right)
$$

can be calculated as follows.

If $\mathrm{d} \pi: \mathfrak{g} \rightarrow \operatorname{End}\left(\mathcal{H}^{\infty}\right)$ is the map obtained from the representation $\mathrm{d} \widehat{\pi}$ via $\mathrm{d} \pi(x):=\mathrm{d} \widehat{\pi}(0, x)$, then we have for each unit vector $v \in \mathcal{H}^{\infty}$ the relation

$$
[\mathrm{d} \pi(x), \mathrm{d} \pi(y)] v=\mathrm{d} \pi([x, y]) v+i \eta(x, y) v,
$$

so that

$$
\begin{aligned}
\eta(x, y) & =\operatorname{Im}\langle[\mathrm{d} \pi(x), \mathrm{d} \pi(y)] v, v\rangle-\operatorname{Im}\langle\mathrm{d} \pi([x, y]) v, v\rangle \\
& =2 \operatorname{Im}\langle\mathrm{d} \pi(x) v, \mathrm{~d} \pi(y) v\rangle+i\langle\mathrm{~d} \pi([x, y]) v, v\rangle .
\end{aligned}
$$

\section{B. The Cone of Positive Definite Forms on a Banach Space}

Let $V$ be a Banach space and $\operatorname{Sym}^{2}(V, \mathbb{R})$ be the Banach space of continuous symmetric bilinear maps $\beta: V \times V \rightarrow \mathbb{R}$, endowed with the norm

$$
\|\beta\|:=\sup \{|\beta(v, v)|:\|v\| \leq 1\} .
$$

Clearly, the set $\operatorname{Sym}^{2}(V, \mathbb{R})$ + of positive semidefinite bilinear maps is a closed convex cone in $\operatorname{Sym}^{2}(V, \mathbb{R})$.

Lemma B.1. The cone $\operatorname{Sym}^{2}(V, \mathbb{R})_{+}$has interior points if and only if $V$ is topologically isomorphic to a Hilbert space. If this is the case, then its interior consists of all those positive definite forms $\beta$ for which the norm $\|v\|_{\beta}:=\sqrt{\beta(v, v)}$ is equivalent to the norm on $V$.

Proof. Suppose first that $V$ carries a Hilbert space structure $\beta$. Replacing the original norm by an equivalent Hilbert norm, we may assume that $V$ is a real Hilbert space. Then $\operatorname{Sym}^{2}(V, \mathbb{R})$ can be identified with the space $\operatorname{Sym}(V)$ of symmetric operators on $V$ by assigning to $A \in \operatorname{Sym}(V)$ the form $\beta_{A}(v, v)=\langle A v, w\rangle$, satisfying

$$
\left|\beta_{A}(v, v)\right|=|(A v, v)| \leq\|A\|\|v\|^{2}, \quad \text { so that }\left\|\beta_{A}\right\| \leq\|A\| .
$$

The polarization identity

$$
(A v, w)=\beta_{A}(v, w)=\frac{1}{4}\left(\beta_{A}(v+w, v+w)-\beta_{A}(v-w, v-w)\right)
$$


further implies that $\|A\| \leq 2 \beta_{A}$, so that the Banach spaces $\operatorname{Sym}(V)$ and $\operatorname{Sym}^{2}(V, \mathbb{R})$ are topologically isomorphic. The identity $\mathrm{id}_{V}=\mathbf{1}$ is an interior point in the cone of positive operators, $\operatorname{Sym}^{2}(V, \mathbb{R})_{+}$has interior points.

Suppose, conversely, that $\beta \in \operatorname{Sym}^{2}(V, \mathbb{R})_{+}$is an interior point. Then $\beta-\operatorname{Sym}^{2}(V, \mathbb{R})_{+}$is a 0-neighborhood in $\operatorname{Sym}^{2}(V, \mathbb{R})$, which implies the existence of some $c>0$ such that $\|\gamma\| \leq c$ implies $\gamma(v, v) \leq \beta(v, v)$ for all $v \in V$. Fixing $v_{0} \in V$, we pick $\alpha \in V^{\prime}$ with $\|\alpha\|=1$ and $\alpha\left(v_{0}\right)=\left\|v_{0}\right\|$. Then $\gamma(v, w):=c \alpha(v) \alpha(w)$ is a symmetric bilinear form with $\|\gamma\|=c\|\alpha\|^{2}=c$. We therefore obtain

$$
c\left\|v_{0}\right\|^{2}=\gamma\left(v_{0}, v_{0}\right) \leq \beta\left(v_{0}, v_{0}\right) \leq\|\beta\|\left\|v_{0}\right\|^{2},
$$

showing that $\beta$ is positive definite and that the norm $\sqrt{\beta(v, v)}$ is equivalent to the norm on $V$.

Remark B.2. Let $(V, \omega)$ be a weakly symplectic Banach space, i.e. $\omega$ is a nondegenerate skew-symmetric bilinear form. Then

$$
\mathfrak{s p}(V, \omega):=\{X \in B(V):(\forall v, w \in V) \omega(X v, w)+\omega(v, X w)=0\}
$$

is the corresponding symplectic Lie algebra. In particular, $X \in B(V)$ belongs to $\mathfrak{s p}(V, \omega)$ if and only if the bilinear form $\omega(X v, w)$ is symmetric. The corresponding quadratic function $H_{X}(v):=\frac{1}{2} \omega(X v, v)$ is called the Hamiltonian function defined by $X$.

If there exists an $X \in \mathfrak{s p}(V, \omega)$ for which $(v, w):=\omega(X v, w)$ defines a Hilbert space structure on $V$, then each continuous linear functional $\alpha \in V^{\prime}$ is of the form $i_{v} \omega$ for some $v \in V$. This means that $(V, \omega)$ is strongly symplectic, i.e. the map $\Phi_{\omega}: V \rightarrow V^{\prime}, v \mapsto i_{v} \omega$ is surjective, hence a topological isomorphism by the Open Mapping Theorem. We further see that $X$ is injective. To see that it is also surjective, let $u \in V$ and represent the continuous linear functional $i_{u} \omega$ as $(w, \cdot)$ for some $w \in V$. Then $\omega(u, v)=(w, v)=\omega(X w, v)$ for all $v \in V$, and thus $X w=u$. Hence $X$ is a topological isomorphism, satisfying $\omega(u, v)=\left(X^{-1} u, v\right)$ for $v, w \in V$. This further implies that $X$ is skew-symmetric.

Proposition B.3. Let $(V, \omega)$ be a strongly symplectic Banach space. Then the convex cone $\left\{X \in \mathfrak{s p}(V, \omega): H_{X} \geq 0\right\}$ has interior points if and only if $V$ is topologically isomorphic to a Hilbert space. If this is the case, then there exists a complex structure $I \in \mathfrak{s p}(V, \omega)$ for which $H_{I}$ defines a compatible Hilbert space structure on $V$, i.e.

$$
\omega(v, w)=\operatorname{Im}\langle v, w\rangle
$$

for the underlying complex Hilbert space.

Proof. (cf. [1, Theorem 3.1.19]) If $\beta: V \times V \rightarrow \mathbb{R}$ is a symmetric bilinear form, then $\Phi_{\beta}: V \rightarrow V^{\prime}, v \mapsto i_{v} \beta$ also is a continuous linear map, and $B:=\Phi_{\omega}^{-1} \circ \Phi_{\beta}: V \rightarrow V$ is continuous linear with

$$
\omega(B v, v)=\Phi_{\omega}(B v)(v)=\Phi_{\beta}(v)(v)=\beta(v, v),
$$


so that every symmetric bilinear form can be represented by an element of $\mathfrak{s p}(V, \omega)$, and we obtain a topological isomorphism $\mathfrak{s p}(V, \omega) \cong \operatorname{Sym}^{2}(V, \mathbb{R})$. Combining this with Lemma B.1 proves the first assertion.

We now assume that $V$ is a real Hilbert space with the scalar product $(v, w)=$ $\omega(X v, w)$, where $X$ is as above. Then $A:=X^{-1}$ is an invertible skew-symmetric operator with $\omega(v, w)=(A v, w)$. Then the complex linear extension $A_{\mathbb{C}}$ to the complex Hilbert space $V_{\mathbb{C}}$ yields a self-adjoint operator $i A_{\mathbb{C}}$. The complex conjugation $\sigma$ of $V_{\mathbb{C}}$ with respect to $V$ now satisfies $\sigma \circ i A_{\mathbb{C}} \circ \sigma=-i A_{\mathbb{C}}$, so that $\operatorname{Spec}\left(i A_{\mathbb{C}}\right)$ is a compact symmetric subset of $\mathbb{R}$, not containing 0 . We therefore have an orthogonal decomposition $V_{\mathbb{C}}=V_{+} \oplus V_{-}$into the positive and negative spectral subspaces of $i A_{\mathbb{C}}$. Since $\sigma\left(V_{ \pm}\right)=V_{\mp}$, we obtain an isomorphism $V \cong V_{+}$, and hence a complex structure $I$ on $V$, corresponding to multiplication by $i$ on $V_{+}$. This means that $v_{+} \in V_{+}$with $v=v_{+}+\sigma\left(v_{+}\right)$satisfies

$$
\begin{aligned}
\omega(I v, v) & =(A I v, v)=\left(A I\left(v_{+}+\sigma\left(v_{+}\right)\right), v_{+}+\sigma\left(v_{+}\right)\right) \\
& =\left(A_{\mathbb{C}}\left(i v_{+}-i \sigma\left(v_{+}\right)\right), v_{+}+\sigma\left(v_{+}\right)\right)=i\left(A_{\mathbb{C}}\left(v_{+}-\sigma\left(v_{+}\right)\right), v_{+}+\sigma\left(v_{+}\right)\right) \\
& =i\left(\left(A_{\mathbb{C}} v_{+}, v_{+}\right)-\left(v_{+}, A_{\mathbb{C}} v_{+}\right)\right)=2\left(i A_{\mathbb{C}} v_{+}, v_{+}\right),
\end{aligned}
$$

so we obtain a complex structure $I$ on $V$ for which the Hamiltonian $H_{I}$ defines a Hilbert structure on $V$. Since $I$ is skew-symmetric, $(V, I)$ inherits the structure of a complex Hilbert space with respect to the scalar product

$$
\langle v, w\rangle:=\omega(I v, w)+i \omega(I v, I w)=\omega(I v, w)+i \omega(v, w) .
$$

Therefore our assumption leads to the representation of $\omega$ as $\omega(v, w)=\operatorname{Im}\langle v, w\rangle$ for a complex Hilbert space structure on $V$.

\section{Involutive Lie Algebras with Root Decomposition}

Definition C.1. (a) We call an Abelian subalgebra $\mathfrak{t}$ of the real Lie algebra $\mathfrak{g}$ a compactly embedded Cartan subalgebra if $\mathfrak{t}$ is maximal Abelian and ad $\mathfrak{t}$ is simultaneously diagonalizable on the complexification $\mathfrak{g}_{\mathbb{C}}$ with purely imaginary eigenvalues. Then we have a root decomposition

$$
\mathfrak{g}_{\mathbb{C}}=\mathfrak{t}_{\mathbb{C}}+\sum_{\alpha \in \Delta} \mathfrak{g}_{\mathbb{C}}^{\alpha},
$$

where $\mathfrak{g}_{\mathbb{C}}^{\alpha}=\left\{x \in \mathfrak{g}_{\mathbb{C}}:\left(\forall h \in \mathfrak{t}_{\mathbb{C}}\right)[h, x]=\alpha(h) x\right\}$ and

$$
\Delta:=\left\{\alpha \in \mathfrak{t}_{\mathbb{C}}^{*} \backslash\{0\}: \mathfrak{g}_{\mathbb{C}}^{\alpha} \neq\{0\}\right\}
$$

is the corresponding root system.

If $\sigma: \mathfrak{g}_{\mathbb{C}} \rightarrow \mathfrak{g}_{\mathbb{C}}$ denotes the complex conjugation with respect to $\mathfrak{g}$, we write $x^{*}:=-\sigma(x)$ for $x \in \mathfrak{g}_{\mathbb{C}}$, so that $\mathfrak{g}=\left\{x \in \mathfrak{g}_{\mathbb{C}}: x^{*}=-x\right\}$. We then have

(I1) $\alpha(x) \in \mathbb{R}$ for $x \in i$.

(I2) $\sigma\left(\mathfrak{g}_{\mathbb{C}}^{\alpha}\right)=\mathfrak{g}_{\mathbb{C}}^{-\alpha}$ for $\alpha \in \Delta$. 
Lemma C.2. For $0 \neq x_{\alpha} \in \mathfrak{g}_{\mathbb{C}}^{\alpha}$ the subalgebra $\mathfrak{g}_{\mathbb{C}}\left(x_{\alpha}\right):=\operatorname{span}\left\{x_{\alpha}, x_{\alpha}^{*},\left[x_{\alpha}, x_{\alpha}^{*}\right]\right\}$ is $\sigma$-invariant and of one of the following types:

(A) The Abelian type: $\left[x_{\alpha}, x_{\alpha}^{*}\right]=0$, i.e. $\mathfrak{g}_{\mathbb{C}}\left(x_{\alpha}\right)$ is two-dimensional Abelian.

(N) The nilpotent type: $\left[x_{\alpha}, x_{\alpha}^{*}\right] \neq 0$ and $\alpha\left(\left[x_{\alpha}, x_{\alpha}^{*}\right]\right)=0$, i.e. $\mathfrak{g}_{\mathbb{C}}\left(x_{\alpha}\right)$ is a threedimensional Heisenberg algebra.

(S) The simple type: $\alpha\left(\left[x_{\alpha}, x_{\alpha}^{*}\right]\right) \neq 0$, i.e. $\mathfrak{g}_{\mathbb{C}}\left(x_{\alpha}\right) \cong \mathfrak{s l}_{2}(\mathbb{C})$. In this case we distinguish the two cases:

(CS) $\alpha\left(\left[x_{\alpha}, x_{\alpha}^{*}\right]\right)>0$, i.e. $\mathfrak{g}_{\mathbb{C}}\left(x_{\alpha}\right) \cap \mathfrak{g} \cong \mathfrak{s u}_{2}(\mathbb{C})$, and

(NS) $\alpha\left(\left[x_{\alpha}, x_{\alpha}^{*}\right]\right)<0$, i.e. $\mathfrak{g}_{\mathbb{C}}\left(x_{\alpha}\right) \cap \mathfrak{g} \cong \mathfrak{s u}_{1,1}(\mathbb{C}) \cong \mathfrak{s l}_{2}(\mathbb{R})$.

Proof. First we note that, in view of $x_{\alpha}^{*} \in \mathfrak{g}_{\mathbb{C}}^{-\alpha}$, [59, Lemma I.2] applies, and we see that $\mathfrak{g}_{\mathbb{C}}\left(x_{\alpha}\right)$ is of one of the three types $(\mathrm{A}),(\mathrm{N})$ and $(\mathrm{S})$. We note that $\alpha\left(\left[x_{\alpha}, x_{\alpha}^{*}\right]\right) \in \mathbb{R}$ because of (I2) and $\left[x_{\alpha}, x_{\alpha}^{*}\right] \in i \mathrm{t}$. Now it is easy to check that $\mathfrak{g}_{\mathbb{C}}\left(x_{\alpha}\right) \cap \mathfrak{g}$ is of type (CS), resp., (NS), according to the sign of this number.

The following proposition provides useful information for the analysis of invariant cones and orbit projections. Here we write $p_{\mathfrak{t}}: \mathfrak{g} \rightarrow \mathfrak{t}$ for the projection along $[\mathfrak{t}, \mathfrak{g}]=\sum_{\alpha}\left(\mathfrak{g}_{\mathbb{C}}^{\alpha}+\mathfrak{g}_{\mathbb{C}}^{-\alpha}\right) \cap \mathfrak{g}$, and $p_{\mathfrak{t}^{*}}: \mathfrak{g}^{*} \rightarrow \mathfrak{t}^{*}$ for the restriction map.

Proposition C.3. Let $x \in \mathfrak{t}, x_{\alpha} \in \mathfrak{g}_{\mathbb{C}}^{\alpha}$ and $\lambda \in \mathfrak{t}^{*}$. Then the following assertions hold:

(i) $p_{\mathfrak{t}}\left(e^{\mathbb{R a d}\left(x_{\alpha}-x_{\alpha}^{*}\right)} x\right)=x+ \begin{cases}\mathbb{R}^{+} \alpha(x)\left[x_{\alpha}^{*}, x_{\alpha}\right] & \text { for } \alpha\left(\left[x_{\alpha}, x_{\alpha}^{*}\right]\right) \leq 0, \\ {[0,2] \frac{\alpha(x)}{\alpha\left(\left[x_{\alpha}, x_{\alpha}^{*}\right]\right)}\left[x_{\alpha}^{*}, x_{\alpha}\right]} & \text { for } \alpha\left(\left[x_{\alpha}, x_{\alpha}^{*}\right]\right)>0 .\end{cases}$

(ii) $p_{\mathfrak{t}^{*}}\left(e^{\operatorname{Rad}^{*}\left(x_{\alpha}-x_{\alpha}^{*}\right)} \lambda\right)=\lambda+ \begin{cases}\mathbb{R}^{+} \lambda\left(\left[x_{\alpha}^{*}, x_{\alpha}\right]\right) \alpha & \text { for } \alpha\left(\left[x_{\alpha}, x_{\alpha}^{*}\right]\right) \leq 0, \\ {[0,2] \frac{\lambda\left(\left[x_{\alpha}^{*}, x_{\alpha}\right]\right)}{\alpha\left(\left[x_{\alpha}, x_{\alpha}^{*}\right]\right)} \alpha} & \text { for } \alpha\left(\left[x_{\alpha}, x_{\alpha}^{*}\right]\right)>0 .\end{cases}$

Proof. (i) is an immediate consequence of [60, Lemma VII.2.9], and (ii) follows from (i) and the relation $p_{\mathrm{t}^{*}}(\lambda)\left(e^{\operatorname{ad} y} x\right)=\lambda\left(p_{\mathfrak{t}}\left(e^{\operatorname{ad} y} x\right)\right)$.

\section{Some Facts on Fock Spaces}

Let $\mathcal{H}$ be a complex Hilbert space. We endow the $n$-fold tensor product with the canonical Hilbert structure defined by

$$
\left\langle v_{1} \otimes \cdots \otimes v_{n}, w_{1} \otimes \cdots \otimes w_{n}\right\rangle:=\prod_{j=1}^{n}\left\langle v_{j}, w_{j}\right\rangle,
$$

and form the Hilbert space direct sum $T(\mathcal{H}):=\widehat{\bigoplus}_{n \in \mathbb{N}_{0}} \mathcal{H}^{\otimes n}$. In $\mathcal{H}^{\otimes n}$ we write $S^{n}(\mathcal{H})$ for the closed subspace generated by the symmetric tensors and $\Lambda^{n}(\mathcal{H})$ for the closed subspace generated by the alternating tensors. We thus obtain subspaces

$$
S(\mathcal{H}):=\widehat{\bigoplus}_{n \in \mathbb{N}_{0}} S^{n}(\mathcal{H}) \text { and } \Lambda(\mathcal{H}):=\widehat{\bigoplus}_{n \in \mathbb{N}_{0}} \Lambda^{n}(\mathcal{H})
$$


of $T(\mathcal{H})$ and write $P_{s}$, resp., $P_{a}$ for the corresponding orthogonal projections:

$$
P_{s}\left(f_{1} \otimes \cdots \otimes f_{n}\right)=\frac{1}{n !} \sum_{\sigma \in S_{n}} f_{\sigma(1)} \otimes \cdots \otimes f_{\sigma(n)}
$$

and

$$
P_{a}\left(f_{1} \otimes \cdots \otimes f_{n}\right)=\frac{1}{n !} \sum_{\sigma \in S_{n}} \operatorname{sgn}(\sigma) f_{\sigma(1)} \otimes \cdots \otimes f_{\sigma(n)} .
$$

The dense subspace $S(\mathcal{H})_{0}:=\sum_{n \geq 0} S^{n}(\mathcal{H})$ of $S(\mathcal{H})$ carries an associative algebra structure, given by

$$
f_{1} \vee \cdots \vee f_{n}:=\sqrt{n !} P_{s}\left(f_{1} \otimes \cdots \otimes f_{n}\right)
$$

and likewise $\Lambda(\mathcal{H})_{0}:=\sum_{n \geq 0} \Lambda^{n}(\mathcal{H})$ inherits an algebra structure defined by

$$
f_{1} \wedge \cdots \wedge f_{n}:=\sqrt{n !} P_{a}\left(f_{1} \otimes \cdots \otimes f_{n}\right) .
$$

A unit vector $\Omega$ in the one-dimensional space $S^{0}(\mathcal{H})=\Lambda^{0}(\mathcal{H})$ is called a vacuum vector.

Lemma D.1. We have

$$
T \vee S=\sqrt{\left(\begin{array}{c}
n+m \\
n
\end{array}\right)} P_{s}(T \otimes S) \quad \text { for } T \in S^{n}(\mathcal{H}), S \in S^{m}(\mathcal{H})
$$

and

$$
T \wedge S=\sqrt{\left(\begin{array}{c}
n+m \\
n
\end{array}\right)} P_{a}(T \otimes S) \quad \text { for } T \in \Lambda^{n}(\mathcal{H}), S \in \Lambda^{m}(\mathcal{H}) .
$$

Proof. For the symmetric case we first note that $f^{n}=\sqrt{n !} f^{\otimes n}$, so that

$$
f^{n} \vee g^{m}=\sqrt{(n+m) !} P_{s}\left(f^{\otimes n} \otimes g^{\otimes m}\right)=\sqrt{\left(\begin{array}{c}
n+m \\
n
\end{array}\right)} P_{s}\left(f^{n} \otimes g^{m}\right) .
$$

Since the elements $f^{n}$ generate $S^{n}(\mathcal{H})$ topologically, (44) follows.

For the alternating case we obtain for $T=f_{1} \wedge \cdots \wedge f_{n}$ and $S=g_{1} \wedge \cdots \wedge g_{m}$ the relation

$$
\begin{aligned}
\frac{1}{\sqrt{(n+m) !}} T \wedge S & =P_{a}\left(f_{1} \otimes \cdots \otimes g_{m}\right) \\
& =P_{a}\left(P_{a}\left(f_{1} \otimes \cdots \otimes f_{n}\right) \otimes P_{a}\left(g_{1} \otimes \cdots \otimes g_{m}\right)\right) \\
& =\frac{1}{\sqrt{n ! m !}} P_{a}(T \otimes S) .
\end{aligned}
$$

Remark D.2. (a) For the norms of the product of $T \in S^{n}(\mathcal{H})$ and $S \in S^{m}(\mathcal{H})$, we obtain with (44)

$$
\frac{1}{\sqrt{(n+m) !}}\|T \vee S\|=\frac{1}{\sqrt{n !} \sqrt{m !}}\left\|P_{s}(T \otimes S)\right\| \leq \frac{1}{\sqrt{n !} \sqrt{m !}}\|T \otimes S\|=\frac{\|T\|}{\sqrt{n !}} \frac{\|S\|}{\sqrt{m !}} .
$$


From that we derive for $T \in S^{k}(\mathcal{H})$ and $n \in \mathbb{N}$ the relation

$$
\frac{1}{\sqrt{(k n) !}}\left\|T^{n}\right\| \leq\left(\frac{1}{\sqrt{k !}}\|T\|\right)^{n}
$$

and for $T \in S^{2}(\mathcal{H})$ we find in particular

$$
\left\|e^{T}\right\|^{2}=\sum_{n=0}^{\infty} \frac{1}{(n !)^{2}}\left\|T^{n}\right\|^{2} \leq \sum_{n=0}^{\infty} \frac{(2 n) !}{(n !)^{2} 2^{n}}\|T\|^{2 n}
$$

which converges for $\|T\|^{2}<\frac{1}{2}$.

(b) For $T \in \Lambda^{n}(\mathcal{H})$ and $S \in \Lambda^{m}(\mathcal{H})$ we likewise obtain with (45)

$$
\frac{1}{\sqrt{(n+m) !}}\|T \wedge S\| \leq \frac{\|T\|}{\sqrt{n !}} \frac{\|S\|}{\sqrt{m !}}
$$

which leads for $T \in \Lambda^{2}(\mathcal{H})$ to

$$
\left\|e^{T}\right\|^{2}=\sum_{n=0}^{\infty} \frac{1}{(n !)^{2}}\left\|T^{n}\right\|^{2} \leq \sum_{n=0}^{\infty} \frac{(2 n) !}{(n !)^{2} 2^{n}}\|T\|^{2 n}
$$

Lemma D.3. Let $A$ be an antilinear Hilbert-Schmidt operator on $\mathcal{H}$ and define $A^{*}$ by $\left\langle A^{*} v, w\right\rangle=\langle A w, v\rangle$. If $A^{*}=A$, then there exists a unique element $\widehat{A} \in$ $S^{2}(\mathcal{H})$ with

$$
\left\langle\widehat{A}, f_{1} \vee f_{2}\right\rangle=\left\langle A f_{1}, f_{2}\right\rangle f \quad \text { for } f_{1}, f_{2} \in \mathcal{H}
$$

and if $A^{*}=-A$, there exists a unique element $\widehat{A} \in \Lambda^{2}(\mathcal{H})$ with

$$
\left\langle\widehat{A}, f_{1} \wedge f_{2}\right\rangle=\left\langle A f_{1}, f_{2}\right\rangle \quad \text { for } f_{1}, f_{2} \in \mathcal{H}
$$

Moreover, the following assertions hold:

(i) $\|\widehat{A}\|^{2}=\frac{1}{2}\|A\|_{2}^{2}$.

(ii) If $A$ and $B$ are hermitian and antilinear, then $\langle\widehat{A}, \widehat{B}\rangle=\frac{1}{2} \operatorname{tr}(A B)$.

(iii) If $A$ and $B$ are skew-hermitian and antilinear, then $\langle\widehat{A}, \widehat{B}\rangle=-\frac{1}{2} \operatorname{tr}(A B)$.

Proof. (a) First we consider the case where $A^{*}=A$. Let $\left(e_{j}\right)_{j \in J}$ be an orthonormal basis of $\mathcal{H}$. Then we have in $S^{2}(\mathcal{H})$ the relations $\left\|e_{j}^{2}\right\|^{2}=2\left\|e_{j} \otimes e_{j}\right\|^{2}=2 \quad$ and $\quad\left\|e_{j} \vee e_{k}\right\|^{2}=2\left\|\frac{1}{2}\left(e_{j} \otimes e_{k}+e_{k} \otimes e_{j}\right)\right\|^{2}=1, \quad j \neq k$.

If $<$ denotes a linear order on $J$, we thus obtain the orthonormal basis

$$
\frac{1}{\sqrt{2}} e_{j}^{2}, \quad e_{j} \vee e_{k}, \quad j<k, \quad \text { of } S^{2}(\mathcal{H})
$$


This leads to

$$
\begin{aligned}
\|\widehat{A}\|^{2} & =\sum_{j} \frac{1}{2}\left|\left\langle\widehat{A}, e_{j}^{2}\right\rangle\right|^{2}+\sum_{j<k}\left|\left\langle\widehat{A}, e_{j} \vee e_{k}\right\rangle\right|^{2} \\
& =\sum_{j} \frac{1}{2}\left|\left\langle A e_{j}, e_{j}\right\rangle\right|^{2}+\sum_{j<k}\left|\left\langle A e_{j}, e_{k}\right\rangle\right|^{2}=\frac{1}{2}\|A\|_{2}^{2} .
\end{aligned}
$$

For $A^{*}=-A$ we similarly get

$$
\|\widehat{A}\|^{2}=\sum_{j<k}\left|\left\langle\widehat{A}, e_{j} \vee e_{k}\right\rangle\right|^{2}=\sum_{j<k}\left|\left\langle A e_{j}, e_{k}\right\rangle\right|^{2}=\frac{1}{2}\|A\|_{2}^{2} .
$$

(b) Let $\operatorname{Herm}_{2}(\mathcal{H})_{a}$ denote the complex subspace of hermitian antilinear HilbertSchmidt operators. Then the prescription $\langle A, B\rangle:=\operatorname{tr}(A B)=\operatorname{tr}\left(A B^{*}\right)$ defines a sesquilinear form on this space with

$$
\overline{\operatorname{tr}(A B)}=\operatorname{tr}\left((A B)^{*}\right)=\operatorname{tr}\left(B^{*} A^{*}\right)=\operatorname{tr}(B A),
$$

so that it is hermitian. Its restriction to the diagonal satisfies

$$
\langle A, A\rangle=\operatorname{tr}\left(A^{2}\right)=\sum_{j \in J}\left\langle A^{2} e_{j}, e_{j}\right\rangle=\sum_{j \in J}\left\langle A e_{j}, A e_{j}\right\rangle=\|A\|_{2}^{2} .
$$

In view of (a), polarization implies that $\operatorname{tr}(A B)=\langle A, B\rangle=2\langle\widehat{A}, \widehat{B}\rangle$ for $A, B \in$ $\operatorname{Herm}_{2}(\mathcal{H})_{a}$.

(c) For the space $\operatorname{Aherm}_{2}(\mathcal{H})_{a}$ of skew-hermitian antilinear Hilbert-Schmidt operators the same argument works with $\langle A, B\rangle:=-\operatorname{tr}(A B)=\operatorname{tr}\left(A B^{*}\right)$.

\section{Acknowledgment}

This work was supported by DFG-grant NE 413/7-1, Schwerpunktprogramm "Darstellungstheorie". We thank Christoph Zellner and Stéphane Merigon for reading large portions of this manuscript.

\section{References}

1. R. Abraham and J. E. Marsden, Foundations of Mechanics, 2nd edn. (Benjamin/ Cummings, 1978).

2. H. Araki and W. Wyss, Representations of the canonical anticommutation relations, Helv. Phys. Acta 37 (1964) 136-159.

3. M. F. Atiyah and A. N. Pressley, Convexity and loop groups, in Arithmetic and Geometry, Vol. II (Birkhäuser, 1983), pp. 33-63.

4. B. Bakalov, N. M. Nikolov, K.-H. Rehren and I. Todorov, Unitary positive energy representations of scalar bilocal quantum fields, Comm. Math. Phys. 271 (2007) $223-246$.

5. E. Balslev, J. Manuceau and A. Verbeure, Representations of the anticommutation relations and Bogoliubov transformations, Comm. Math. Phys. 8 (1968) 315-326.

6. D. Beltiţă, Smooth Homogeneous Structures in Operator Theory (Chapman and Hall, 2006). 
7. D. Beltita and K.-H. Neeb, A non-smooth continuous unitary representation of a Banach-Lie group, J. Lie Th. 18 (2008) 933-936.

8. D. Beltita and K.-H. Neeb, Schur-Weyl Theory for $C^{*}$-algebras, in preparation.

9. J. Bochnak and J. Siciak, Analytic functions in topological vector spaces, Stud. Math. 39 (1971) 77-112.

10. H.-J. Borchers, Translation Group and Particle Representations in Quantum Field Theory (Springer, 1996).

11. N. Bourbaki, General Topology (Springer, 1989), Chaps. 1-4.

12. N. Bourbaki, Espaces Vectoriels Topologiques (Springer, 2007), Chaps. 1-5.

13. M. J. Bowick and S. G. Rajeev, The holomorphic geometry of the closed bosonic string theory and Diff $\left(\mathbb{S}^{1}\right) / \mathbb{S}^{1}$, Nucl. Phys. B 293 (1987) 348-384.

14. O. Bratteli and D. W. Robinson, Operator Algebras and Quantum Statistical Mechanics 2, 2nd edn. (Springer, 1997).

15. A. L. Carey, Infinite dimensional groups and quantum field theory, Act. Appl. Math. 1 (1983) 321-333.

16. A. Carey and K. C. Hannabus, Infinite dimensional groups and Riemann surface field theory, Comm. Math. Phys. 176 (1996) 321-351.

17. A. Carey and E. Langmann, Loop groups and quantum fields, in Geometric Analysis and Applications to Quantum Field Theory, eds. P. Bouwknegt and S. Wu, Progr. in Math., Vol. 205 (Birkhäuser, 2002), pp. 45-94.

18. A. Carey and S. N. M. Ruijsenaars, On fermion gauge groups, current algebras, and Kac-Moody algebras, Acta Appl. Math. 10 (1987) 1-86.

19. R. Delbourgo and J. R. Fox, Maximum weight vectors possess minimal uncertainty, J. Phys. A: Math. Gen. 10 (1977) 233-235.

20. J. Dixmier, Les $C^{*}$-algèbres et Leurs Représentations (Gauthier-Villars, 1964).

21. Chr. Fewster and S. Hollands, Quantum energy inequalities in two-dimensional conformal field theory, Rev. Math. Phys. 17 (2005) 577-612.

22. I. M. Gel'fand and N. Ya Vilenkin, Generalized Functions, Vol. 4: Applications of Harmonic Analysis, translated by Amiel Feinstein (Academic Press, 1964).

23. V. Georgescu, On the spectral analysis of quantum field Hamiltonians, arXiv:math$\mathrm{ph} / 0604072 \mathrm{v} 1$.

24. H. Glöckner, Algebras whose groups of units are Lie groups, Stud. Math. 153 (2002) 147-177.

25. H. Glöckner and K.-H. Neeb, Infinite Dimensional Lie Groups, Vol. I, Basic Theory and Main Examples, in preparation.

26. P. Goddard and D. Olive, Kac-Moody and Virasoro algebras in relation to quantum physics, Internat. J. Mod. Phys. A 1 (1986) 303-414.

27. R. Goodman and N. R. Wallach, Structure and unitary cocycle representations of loop groups and the group of diffeomorphisms of the circle, J. Reine Ang. Math. 347 (1984) 69-133.

28. , Projective unitary positive energy representations of Diff $\left(\mathbb{S}^{1}\right)$, J. Funct. Anal. 63 (1985) 299-312.

29. H. Grundling and K.-H. Neeb, Full regularity for a $C^{*}$-algebra of the canonical commutation relations, Rev. Math. Phys., to appear

30. L. Guieu and C. Roger, L'Algèbre et le Groupe de Virasoro: Aspects Géométriques et Algébriques, Généralisations, Les Publications (CRM, 2007).

31. R. Haag and D. Kastler, An algebraic approach to quantum field theory, J. Math. Phys. 5 (1964) 848-861.

32. K. C. Hannabus, On a property of highest weight vectors, Quart. J. Math. 33 (1982) 91-96. 
33. P. de la Harpe, The Clifford algebra and the spinor group of a Hilbert space, Compos. Math. 25 (1972) 245-261.

34. G. C. Hegerfeldt, Gårding domains and analytic vectors for quantum fields, J. Math. Phys. 13 (1972) 821-827.

35. J. Hilgert and K. H. Hofmann, Lorentzian cones in real Lie algebras, Monatsh. Math. 100 (1985) 183-210.

36. J. Hilgert, K. H. Hofmann and J. D. Lawson, Lie Groups, Convex Cones, and Semigroups (Oxford Univ. Press, 1989).

37. J. Hilgert, K.-H. Neeb and W. Plank, Symplectic convexity theorems and coadjoint orbits, Comp. Math. 94 (1994) 129-180.

38. J. Hilgert and G. Ólafsson, Causal Symmetric Spaces, Geometry and Harmonic Analysis (Academy Press, 1996).

39. K. H. Hofmann and S. A. Morris, The Structure of Compact Groups (de Gruyter, 1998).

40. V. G. Kac and D. H. Peterson, Unitary structure in representations of infinite dimensional groups and a convexity theorem, Invent. Math. 76 (1984) 1-14.

41. V. G. Kac and A. K. Raina, Highest Weight Representations of Infinite Dimensional Lie Algebras (World Scientific, 1987).

42. W. Kaup, Über die Klassifikation der symmetrischen hermiteschen Mannigfaltigkeiten unendlicher Dimension I, II, Math. Ann. 257 (1981) 463-486; 262 (1983) $57-75$.

43. W. Kaup, On real Cartan factors, Manuscripta Math. 92 (1997) 191-222.

44. A. Kirillov, Kähler structure on the $K$-orbits of the group of diffeomorphisms of a circle, Funct. Anal. Appl. 21 (1987) 122-125.

45. _ Geometric approach to discrete series of unirreps for Vir, J. Math. Pures Appl. 77 (1998) 735-746.

46. A. A. Kirillov and D. V. Yuriev, Kähler geometry of the infinite-dimensional homogeneous space $M=\operatorname{Diff}_{+}\left(\mathbb{S}^{1}\right) / \operatorname{Rot}\left(\mathbb{S}^{1}\right)$, Funct. Anal. Appl. 21 (1987) 284-294.

47. L Representations of the Virasoro algebra by the orbit method, J. Geom. Phys. 5 (1988) 351-363.

48. B. Kostant and S. Sternberg, The Schwarzian derivative and the conformal geometry of the Lorentz hyperboloid, in Quantum Theories and Geometry, eds. M. Cahen and M. Flato (Kluwer, 1988), pp. 113-1225.

49. E. Langmann, Cocycles for boson and fermion Bogoliubov transformations, J. Math. Phys. 35 (1994) 96-112.

50. L. Lempert, The Virasoro group as a complex manifold, Math. Res. Lett. 2 (1995) 479-495.

51. G. Mack and M. de Riese, Simple space-time symmetries: Generalized conformal field theory, arXiv:hep-th/0410277v2.

52. M. Magyar, Continuous Linear Representations, Math. Studies, Vol. 168 (NorthHolland, 1992).

53. J. E. Marsden and T. Ratiu, Introduction to Mechanics and Symmetry, 2nd edn., Texts in Appl. Math., Vol. 17 (Springer, 1999).

54. A. Medina and P. Revoy, Algèbres de Lie et produit scalaire invariant, Ann. scient. Éc. Norm. Sup. $4^{e}$ série 18 (1985) 533-561.

55. J. Mickelsson, Kac-Moody groups, topology of the Dirac determinant bundle, and fermionization, Comm. Math. Phys. 110 (1987) 173-183.

56. - Current Algebras and Groups (Plenum Press, 1989).

57. J. Milnor, Remarks on infinite dimensional Lie groups, in Relativité, Groupes et Topologie II (Les Houches, 1983), eds. B. DeWitt and R. Stora (North-Holland, 1984), pp. 1007-1057. 
58. K.-H. Neeb, The classification of Lie algebras with invariant cones, J. Lie Th. 4 (1994) 1-47.

59. Holomorphic highest weight representations of infinite dimensional complex classical groups, J. Reine Angew. Math. 497 (1998) 171-222.

60. Holomorphy and Convexity in Lie Theory, Expositions in Mathematics, Vol. 28 (de Gruyter, 1999).

61. Compressions of infinite dimensional bounded symmetric domains, Semigroup Forum 63 (2001) 71-105.

62. $\_$, Representations of infinite dimensional groups in Infinite Dimensional Kähler Manifolds, eds. A. Huckleberry and T. Wurzbacher, DMV-Seminar, Vol. 31 (Birkhäuser, 2001), pp. 131-178.

63. Classical Hilbert-Lie groups, their extensions and their homotopy groups, in Geometry and Analysis on Finite- and Infinite-Dimensional Lie Groups, eds. A. Strasburger et al., Banach Center Publications, Vol. 55 (Warszawa, 2002), pp. $87-151$.

64. Central extensions of infinite-dimensional Lie groups, Ann. l'Inst. Fourier 52 (2002) 1365-1442.

65. Infinite-dimensional Lie groups and their representations, in Lie Theory: Lie Algebras and Representations, Progress in Math., Vol. 228, eds. J. P. Anker and B. Ørsted (Birkhäuser, 2004), pp. 213-328.

66. Towards a Lie theory of locally convex groups, Jpn. J. Math. 3rd Ser. 1 (2006) 291-468.

67. Lie groups, Semigroup Forum 77 (2008) 5-35.

68. Semibounded unitary representations of infinite dimensional Lie groups, in Infinite Dimensional Harmonic Analysis IV, eds. J. Hilgert et al. (World Scientific, 2009), pp. 209-222.

69. Unitary highest weight modules of locally affine Lie algebras, in Proc. of the Workshop on Quantum Affine Algebras, Extended Affine Lie Algebras and Applications (Banff, 2008), eds. Y. Gao et al. (Amer. Math. Soc.), to appear.

70. - Invariant convex cones in infinite dimensional Lie algebras, in preparation.

71. _ On differentiable vectors for representations of infinite dimensional Lie groups, in preparation.

72. K.-H. Neeb and B. Ørsted, Unitary highest weight representations in Hilbert spaces of holomorphic functions on infinite dimensional domains, J. Funct. Anal. 156 (1998) 263-300.

73. Y. Neretin, Holomorphic extension of representations of the group of diffeomorphisms of the circle, Math. USSR Sbor. 67 (1990) 75-97.

74. A. Neumann, The classification of symplectic structures of convex type, Geom. Dedicata 79 (2000) 299-320.

75. An infinite dimensional version of the Kostant Convexity Theorem, J. Funct. Anal. 189 (2002) 80-131.

76. G. I. Ol'shanskiur, Invariant cones in Lie algebras, Lie semigroups, and the holomorphic discrete series, Funct. Anal. Appl. 15 (1982) 275-285.

77. J. T. Ottesen, Infinite Dimensional Groups and Algebras in Quantum Physics, Lecture Notes in Physics, Vol. 27 (Springer, 1995).

78. V. Yu. Ovsienko, Coadjoint representation of Virasoro-type Lie algebras and differential operators on tensor-densities, in Infinite Dimensional Kähler Manifolds (Oberwolfach, 1995), DMV Sem., Vol. 31 (Birkhäuser, 2001), pp. 231-255. 
79. G. K. Pedersen, $C^{*}$-Algebras and Their Automorphism Groups (Academic Press, 1989).

80. R. T. Powers and E. Størmer, Free states of the canonical anticommutation relations, Comm. Math. Phys. 16 (1970) 1-33.

81. A. Pressley and G. Segal, Loop Groups (Oxford Univ. Press, 1986).

82. M. C. Reed, A Gårding domain for quantum fields, Comm. Math. Phys. 14 (1969) 336-346.

83. S. Reed and B. Simon, Methods of Modern Mathematical Physics I: Functional Analysis (Academic Press, 1973).

84. S. N. M. Ruijsenaars, On Bogoliubov transformations for systems of relativistic charged particles, J. Math. Phys. 18 (1977) 517-526.

85. Y. S. Samoilenko, Spectral Theory of Families of Self-Adjoint Operators (Kluwer, 1991).

86. M. Schottenloher, A Mathematical Introduction to Conformal Field Theory, Lecture Notes in Physics, Vol. 43 (Springer, 1997).

87. L. Schwartz, Théorie des Distributions (Hermann, 1973).

88. G. Segal, Unitary representations of some infinite-dimensional groups, Comm. Math. Phys. 80 (1981) 301-342.

89. I. E. Segal, The structure of a class of representations of the unitary group on a Hilbert space, Proc. Amer. Math. Soc. 81 (1957) 197-203.

90. Distributions in Hilbert spaces and canonical systems of operators, Trans. Amer. Math. Soc. 88 (1958) 12-41.

91. Foundations of the theory of dynamical systems of infinitely many degrees of freedom. I, Mat. Fys. Medd. Danske Vid. Selsk. 31 (1959) 1-39.

92. USA 57 (1967) 194-197.

93. _ Mathematical Cosmology and Extragalactical Astronomy (Academic Press, 1976).

94. The complex-wave representation of the free boson field, in Topics in Funct. Anal., Adv. in Math. Suppl. Studies, Vol. 3 (1978), pp. 321-343.

95. I. E. Segal, H. P. Jakobsen, B. Ørsted, B. Speh and M. Vergne, Symmetry and causality properties of physical fields, Proc. Nat. Acad. Sci. USA 75 (1978) 16091611.

96. D. Shale, Linear symmetries of free boson fields, Trans. Amer. Math. Soc. 103 (1962) 146-169.

97. D. Shale and W. F. Stinespring, Spinor representations of infinite orthogonal groups, J. Math. Mech. 14 (1965) 315-322.

98. M. Spera and T. Wurzbacher, Determinants, Pfaffians and quasi-free representations of the CAR algebra, Rev. Math. Phys. 10 (1998) 705-721.

99. M. Thill, Representations of hermitian commutative $*$-algebras by unbounded operators, arXiv:math.OA.0908.3267.

100. V. T. Laredo, Positive energy representations of the loop groups of non-simply connected Lie groups, Comm. Math. Phys. 207 (1999) 307-339.

101. I I I I L J. Funct. Anal. 161 (1999) 478-508.

102. H. Upmeier, Symmetric Banach Manifolds and Jordan $C^{*}$-algebras (North-Holland, 1985).

103. M. Vergne, Groupe symplectique et seconde quantification, C. R. Acad. Sci. Paris 285 (1977) 191-194. 
104. A. M. Vershik, Metagonal groups of finite and infinite dimension, in Representations of Lie Groups and Related Topics, eds. A. M. Vershik and D. P. Zhelobenko (Gordon and Breach, 1990), pp. 1-37.

105. E. B. Vinberg, Invariant cones and orderings in Lie groups, Funct. Anal. Appl. 14 (1980) 1-13. 